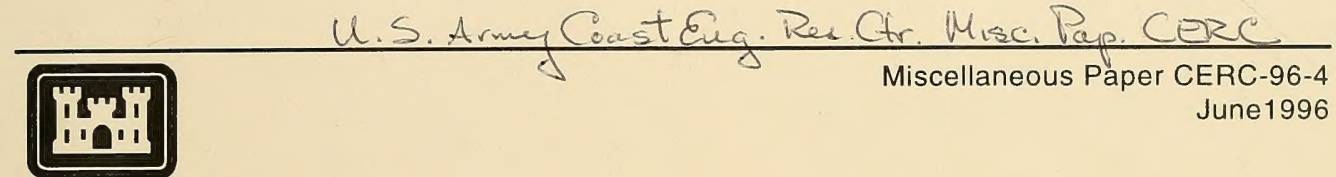

US Army Corps

of Engineers

Waterways Experiment

Station

\title{
1994 Annual Index of Wind Wave Directional Spectra Measured at Harvest Platform
}

by Charles E. Long

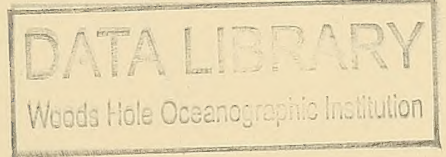

Approved For Public Release; Distribution Is Unlimited

GC

214

.43

196

1996

Prepared for Headquarters, U.S. Army Corps of Engineers 
The contents of this report are not to be used for advertising, publication, or promotional purposes. Citation of trade names does not constitute an official endorsement or approval of the use of such commercial products. 


\section{Annual Index of Wind Wave Directional Spectra Measured at Harvest Platform}

by Charles E. Long

U.S. Army Corps of Engineers

Waterways Experiment Station

3909 Halls Ferry Road

Vicksburg, MS 39180-6199

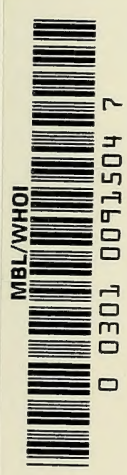

Final report

Approved for public release; distribution is unlimited

Prepared for U.S. Army Corps of Engineers

Washington, DC 20314-1000

Under Civil Works Research Work Unit 32484 


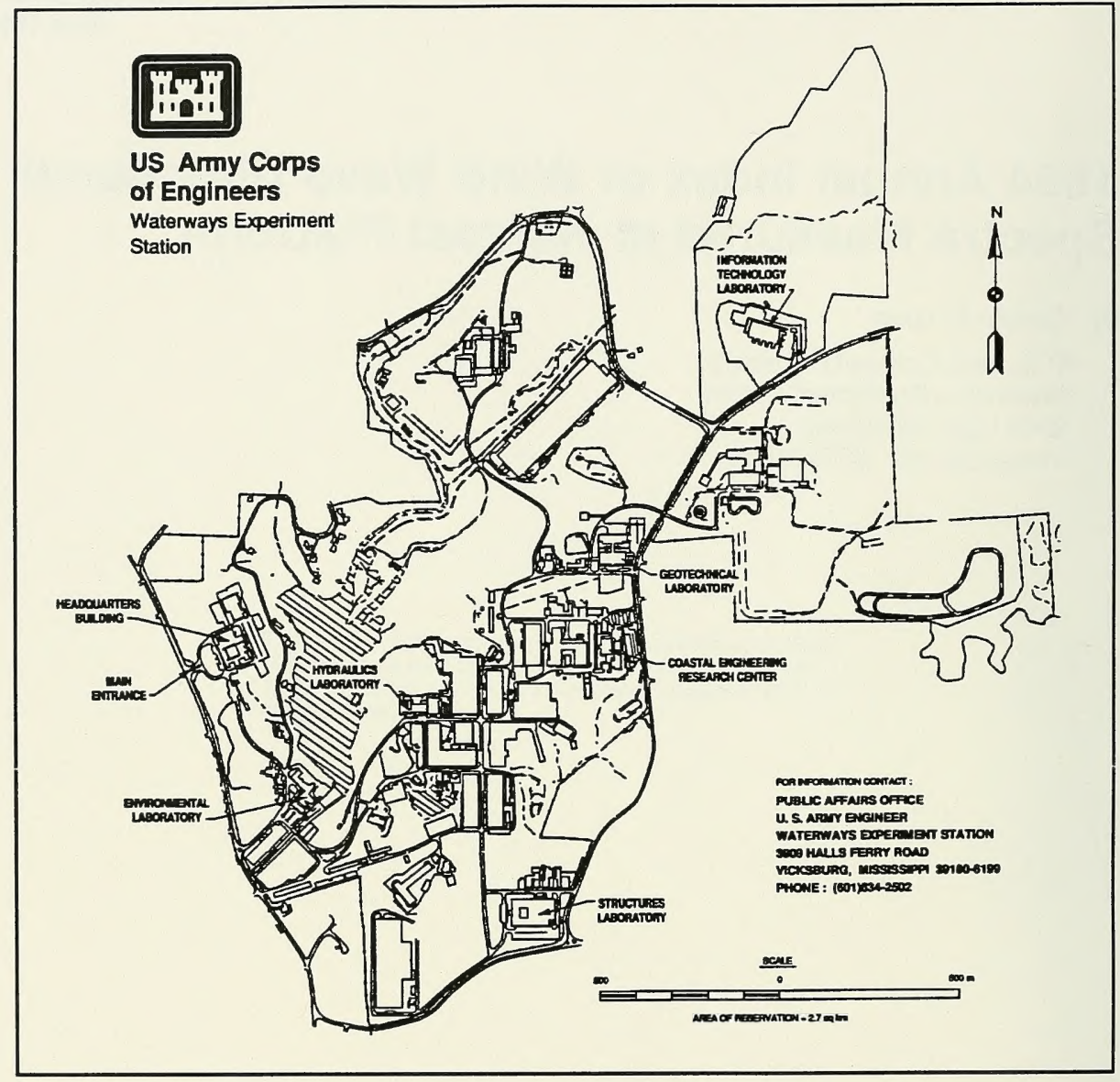

Waterways Experiment Station Cataloging-in-Publication Data

Long, Charles E.

1994 annual index of wind wave directional spectra measured at Harvest Platform / by

Charles E. Long ; prepared for U.S. Army Corps of Engineers.

118 p. : ill. ; $28 \mathrm{~cm}$. - (Miscellaneous paper ; CERC-96-4)

Includes bibliographic references.

1. Wind waves - California - Point Conception - Statistics. 2. Water waves -

California - Point Conception - Statistics. 3. Ocean waves - California - Point

Conception - Statistics. 4. Frequency spectra. I. United States. Army. Corps of

Engineers. II. U.S. Army Engineer Waterways Experiment Station. III. Coastal

Engineering Research Center (U.S. Army Engineer Waterways Experiment Station) IV.

Title. V. Series: Miscellaneous paper (U.S. Army Engineer Waterways Experiment

Station) ; CERC-96-4.

TA7 W34m no.CERC-96-4 


\section{Contents}

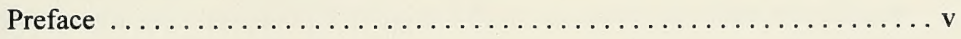

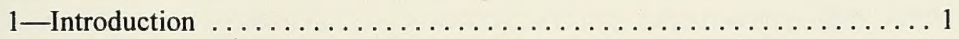

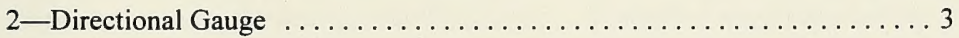

Gauge Location and Array Geometry $\ldots \ldots \ldots \ldots \ldots \ldots \ldots \ldots \ldots \ldots \ldots$

Pressure Gauges and Data Path . . . . . . . . . . . . . . 3

Collection Schedule and Data Set Size $\ldots \ldots \ldots \ldots \ldots \ldots \ldots \ldots$

3-Primary Data Analysis $\ldots \ldots \ldots \ldots \ldots \ldots \ldots \ldots \ldots \ldots \ldots \ldots \ldots \ldots \ldots \ldots \ldots \ldots$

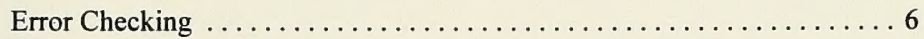

Frequency-Direction Spectra $\ldots \ldots \ldots \ldots \ldots \ldots \ldots \ldots \ldots \ldots \ldots \ldots \ldots \ldots$

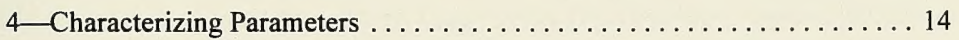

Wave Height, Peak Frequency, and Peak Direction . . . . . . . . 14

Circular Moment Parameters . . . . . . . . . . . . . . . . . . 15

Quartile Parameters ........................... 16

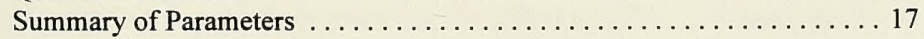

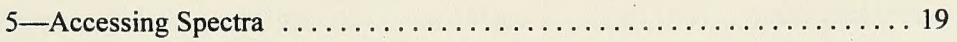

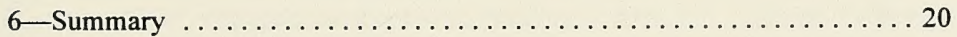

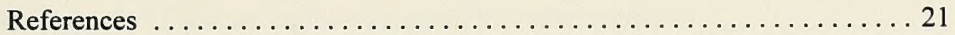

Appendix A: Table of Collection Times and Bulk Parameters ........ Al

Appendix B: Time Series Graphs of Bulk Parameters $\ldots \ldots \ldots \ldots \ldots \ldots$ B1

Appendix C: Listing of FORTRAN Computer Program $\ldots \ldots \ldots \ldots \ldots$ Cl

Appendix D: Listing of Sample Data File $\ldots \ldots \ldots \ldots \ldots \ldots \ldots \ldots \ldots \ldots$ 
Appendix E: Notation

E1

SF 298 


\section{Preface}

This report indexes parameters of and describes means of access to a series of wind wave frequency-direction spectral observations made with a six-element, high-resolution directional wave gauge at Texaco Oil Company's Harvest Platform. The work was motivated by a need to publicize these results so they can be used by all investigators interested in natural wind wave energy distributions at a deepwater site near the exposed California coast. This effort was authorized by Headquarters, U.S. Army Corps of Engineers (HQUSACE), under Civil Works Coastal Navigation Hydrodynamics Program Research Work Unit 32484, "Directionality of Waves in Shallow Water." Funds were provided through the Coastal Engineering Research Center (CERC), U.S. Army Engineer Waterways Experiment Station (WES), under the program management of Ms. Carolyn M. Holmes, CERC. Messrs. John H. Lockhart, Jr., Charles Chesnutt, and Barry W. Holliday were HQUSACE Technical Monitors.

This report was prepared by Dr. Charles E. Long, under the direct supervision of Mr. William A. Birkemeier, Chief, Field Research Facility (FRF), CERC, and Mr. Thomas W. Richardson, Chief, Engineering Development Division (EDD), CERC. General supervision was provided by Dr. James R. Houston and Mr. Charles C. Calhoun, Jr., Director and Assistant Director, CERC, respectively.

Mr. David D. McGehee, Prototype Measurement and Analysis Branch, EDD, CERC, was instrumental in coordinating the efforts of CERC and the State of California in data archiving and gauge maintenance by the Coastal Data Information Program (CDIP) at Scripps Institution of Oceanography (SIO). Data transfer between SIO and the FRF was coordinated under the direction of Dr. Richard J. Seymour, CDIP, with particularly helpful assistance from Ms. Julianna Thomas, CDIP. Ms. Judy H. Roughton, FRF, produced Figure 1 of this report. The contributions of all these individuals are gratefully acknowledged.

At the time of publication of this report, Director of WES was Dr. Robert W. Whalin. Commander was COL Bruce K. Howard, EN.

The contents of this report are not to be used for advertising, publication, or promotional purposes. Citation of trade names does not constitute an official endorsement or approval of the use of such commercial products. 



\section{Introduction}

In late December 1992, a high-resolution directional wave measuring system became fully operational on Texaco Oil Company's Harvest Platform to make long-term observations of the deep-ocean wind wave climate in the vicinity of the Southern California Bight (Figure 1). Such observations are necessary to provide

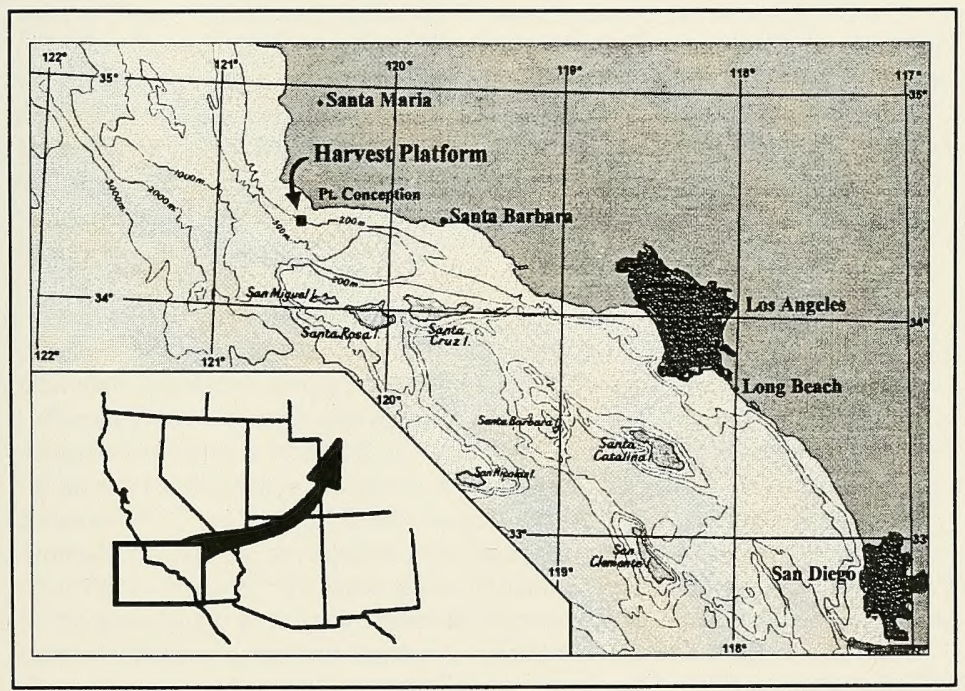

Figure 1. Southern California Bight and location of Harvest Platform

ground truth for interpreting satellite imagery of the ocean surface, test evolution and propagation models of open-ocean wind waves, and establish seaward boundary conditions for models of wave propagation and transformation from deep water to coastal regions. The purpose of this report is to encourage broad use of these observations by parametrically describing 2,320 wind wave frequency-direction spectral estimates obtained in calendar year 1994, and identifying a means whereby an investigator can access these spectra. These results are from the second year of collection. Results from the first year are described by Long (1995a). 
For completeness, this report briefly describes the directional gauge geometry and data collection scheme (Chapter 2 ), error checking procedures and basic directional estimation algorithm (Chapter 3 ), and definitions of parameters used to characterize the observations (Chapter 4). Appendix A contains a table of these characterizing parameters, and acts as an index for the 1994 database. Time series graphs of these parameters are presented in Appendix B. Chapter 5 describes how data can be obtained as well as the data format and file-naming scheme. 


\section{Directional Gauge}

\section{Gauge Location and Array Geometry}

As indicated in Figure 1, Harvest Platform is located about $20 \mathrm{~km}$ (10.8 n.m.) west of Point Conception, California, in water with a mean depth of $202 \mathrm{~m}$ $(663 \mathrm{ft})$. Waves originating in the greater Pacific Ocean can reach the platform via relatively unobstructed paths from the north, west, and south. The mean water depth ensures deepwater wave conditions for waves with lengths shorter than about $400 \mathrm{~m}(1,312 \mathrm{ft})$, or frequencies higher than about $0.06 \mathrm{~Hz}$. Spectra reported herein are processed at frequencies between 0.04 and $0.16 \mathrm{~Hz}$, so it is likely that directional spectra for frequencies between 0.04 and $0.06 \mathrm{~Hz}$ are affected somewhat by refraction.

Directional wave detection is achieved with a spatial array of six subsurface pressure gauges mounted on the Harvest Platform framework. Figure 2 shows a plan view of relative gauge positions, and the array orientation in a geophysical reference frame. Gauge spacing takes advantage of the maximum horizontal dimensions of Harvest Platform, and allows directional estimation for waves in the frequency band noted in the previous paragraph. All gauges are mounted at a depth of $15.72 \mathrm{~m}(51.57 \mathrm{ft})$ below mean sea level, which ensures they will not protrude through the sea surface under extreme wave conditions that have been observed at this site.' To avoid aliasing in directional estimation, the lower resolution wavelength limit is two times the shortest lag spacing of the array. In the Harvest Platform array, this limit is $45.4 \mathrm{~m}$ (149.0 ft), which corresponds to a wave frequency of about $0.18 \mathrm{~Hz}$. Signal analysis used in this report was limited further to $0.16 \mathrm{~Hz}$ to be conservatively clear of aliasing effects.

\section{Pressure Gauges and Data Path}

Individual sensors were Model TJE absolute pressure sensors manufactured by Sensotec Transducer Company with operating ranges of 0 to $100 \mathrm{psia}$ ( 0 to $689.5 \mathrm{kPa}$ ), and a manufacturer's stated accuracy of \pm 0.1 percent of full scale. The six gauges on Harvest Platform were sampled simultaneously at $1 \mathrm{~Hz}$,

\footnotetext{
1 Personal communication, 1991, Dr. R. J. Seymour, Coastal Data Information Program (CDIP), Scripps Institution of Oceanography (SIO).
} 


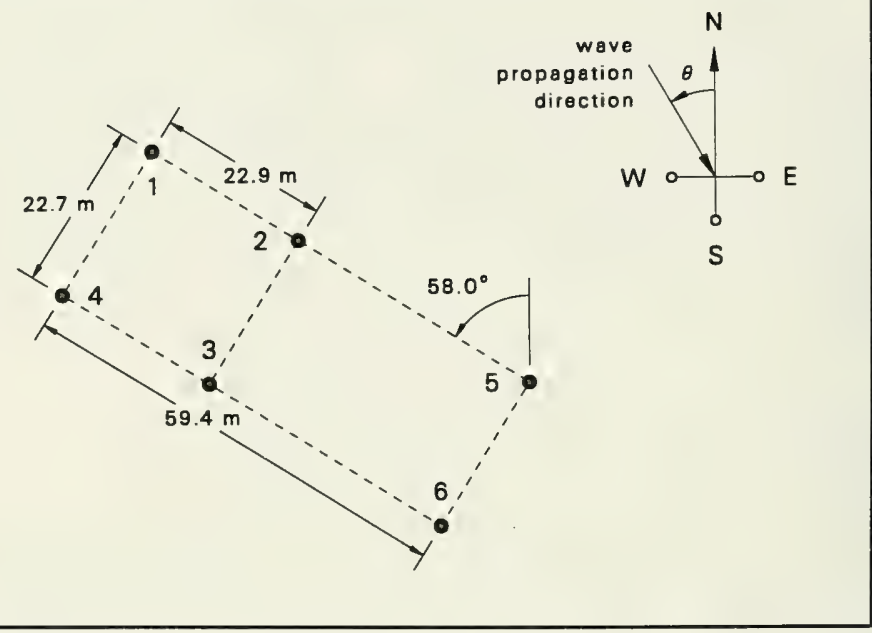

Figure 2. Dimensions and orientation of the Harvest Platform array

digitized, and then fed to a concentrator where the set of samples was buffered. Buffered signals were periodically transmitted to shore through a telephone connection, and ultimately stored as collection files on the main computer of the CDIP, Ocean Engineering Research Group, SIO, La Jolla, CA. Each collection time series is $8,192 \mathrm{sec}(2 \mathrm{hr} 16 \mathrm{~min} 32 \mathrm{sec})$ in length.

Data processing for results presented in this report was not performed at the CDIP site, and so is independent of the processing done and published by that group (Monthly reports, Coastal Data Information Program). Data collections were transferred to the Field Research Facility (FRF) of the U.S. Army Engineer Waterways Experiment Station's Coastal Engineering Research Center for processing by high-resolution techniques that are different from those used by CDIP. Data transfer was accomplished over an electronic network.

\section{Collection Schedule and Data Set Size}

Collections were made eight times daily, at approximately 3 -hr intervals. Nominal collection start times were $0200,0500,0800,1100,1400,1700,2000$, and 2300 GMT. Actual collection start times varied by several minutes on either side of these nominal start times because the amount of time required to establish a phone link varied from collection to collection. There are several periods of several days duration where collections are virtually continuous. These occur when CDIP shifts to a collection mode intended to detect tsunami waves, and result in 12 directional spectral estimates daily instead of the normal eight. 
Of the possible 2,920 collections during calendar year 1994 (assuming eight collections per day), a total of 2,320 collections were acquired and processed as frequency-direction spectra. A number of collections were lost because of the inability to establish or maintain electrically clean phone links to the concentrator on Harvest Platform. An additional number of collections were not processed because data did not satisfy error-checking constraints described in Chapter 3 of this report. 


\section{Primary Data Analysis}

Primary data processing was done by checking data quality through a series of spectral intercomparisons, and, for data of sufficient quality, computing frequency-direction spectra. All steps rely on Fourier analysis of pressure gauge time series data, and subsequent computation of cross-spectral densities. A discussion of error-checking procedures then leads logically to the subsequent steps involved in frequency-direction spectral computation.

\section{Error Checking}

The first step in data processing is computation of discrete estimates of frequency autospectra of pressure signals, and surface-corrected cross-spectral densities of signals from all pairs of gauges. Cross spectra are denoted in complex form as $C_{i j}\left(f_{n}\right)-i Q_{i j}\left(f_{n}\right)$, where $C_{i j}\left(f_{n}\right)$ is the coincident spectrum, $Q_{i j}\left(f_{n}\right)$ is the quadrature spectrum, $i$ and $j$ (as subscripts) are indices ranging in value from 1 to 6 that refer to the gauge numbers shown in Figure 2, and $f_{n}$ is the $n^{\text {th }}$ of a set of $N$ discrete frequencies. ${ }^{1}$ Frequency autospectra are denoted $S\left(f_{n}\right)$, and, if surface corrected with the linear wave pressure response functions (Dean and Dalrymple 1984), are identically equal to $C_{i i}\left(f_{n}\right)$. All spectra are computed using Welch's method (Welch 1967) with standard Fourier analysis techniques (Bendat and Piersol 1971).

In a collection, the $8,192-\mathrm{sec}$ time series from each gauge is analyzed in 15 half-lapped segments of $1,024 \mathrm{sec}$ duration. Each segment is demeaned, tapered with a variance-preserving window, and converted to the frequency domain with a discrete Fourier transform. At this point, the analysis is split into two parts: estimates of pressure autospectra from each gauge at depth, and estimates of surfacecorrected cross spectra of sea surface displacement. Raw cross-spectral estimates are formed for all gauge pairs using temporally corresponding transformed segments of pressure data corrected to represent sea surface displacement. Raw autospectral estimates are formed for each of the 15 transform segments for each individual gauge. At the error-checking stage, autospectral estimates are not surface corrected.

\footnotetext{
${ }^{1}$ For convenience, symbols and abbreviations are listed in the notation (Appendix E).
} 
For both autospectra and cross spectra, smooth estimates are formed by averaging raw estimates over all 15 segments, and averaging results over 10 adjacent frequency bands. Final resolution frequency bandwidth is $d f=0.00977 \mathrm{~Hz}$, and the pass band of frequencies ranges from 0.044 to $0.162 \mathrm{~Hz}$, which corresponds to $(N=) 13$ discrete frequency bands. Degrees of freedom for spectral estimates range from 160 to about 200 , depending on the extent to which the second halves of time series segments are correlated with the first halves (Welch 1967).

\section{Autospectral intercomparisons}

One part of error checking is a graphic intercomparison of signal means and autospectra, an example of which is shown in the lower left graph of Figure 3. Frequency autospectral estimates of data from all six pressure gauges are plotted on the same set of axes from the first resolvable frequency band out to the temporal Nyquist frequency. If a pressure gauge is malfunctioning, its autospectrum will deviate obviously from the main group of curves.

The small inset graph in the lower left graph of Figure 3 is an analysis of signal means. The closely packed group of symbols of nearly constant value represents the deviations of the segment means from the median of the set of segment means for each of the 15 segments. If a gauge develops signal drift problems, it will be obvious as a symbol that deviates from the main group of symbols. Triangle symbols in the small inset graph show the deviation of the indicated water surface from mean sea level (gauge height off the bottom plus median of gauge mean depths for each segment minus the total long-term mean ocean depth of $202 \mathrm{~m}$ ), and is therefore an indication of tide stage at Harvest Platform for each of the 15 segments in a collection.

\section{Coherence and phase comparisons}

The next step in error checking is computation of a dimensionless cross spectrum $M_{i j}\left(f_{n}\right)$, defined by

$$
M_{i j}\left(f_{n}\right)=\frac{C_{i j}\left(f_{n}\right)-i Q_{i j}\left(f_{n}\right)}{\sqrt{C_{i i}\left(f_{n}\right)} \sqrt{C_{j j}\left(f_{n}\right)}}
$$

Equation 1 is used in error checking in the form of coherence and phase estimates.

Coherence of signals from gauges $i$ and $j$ at discrete frequency $f_{n}$ is

$$
\Gamma_{i j}^{2}\left(f_{n}\right)=\left|M_{i j}\left(f_{n}\right)\right|^{2}
$$

Signal phase difference of gauge $i$ relative to gauge $j$ at frequency $f_{n}$ is

$$
\phi_{i j}\left(f_{n}\right)=\tan ^{-1}\left(\frac{\operatorname{Im}\left[M_{i j}\left(f_{n}\right)\right]}{\operatorname{Re}\left[M_{i j}\left(f_{n}\right)\right]}\right)
$$




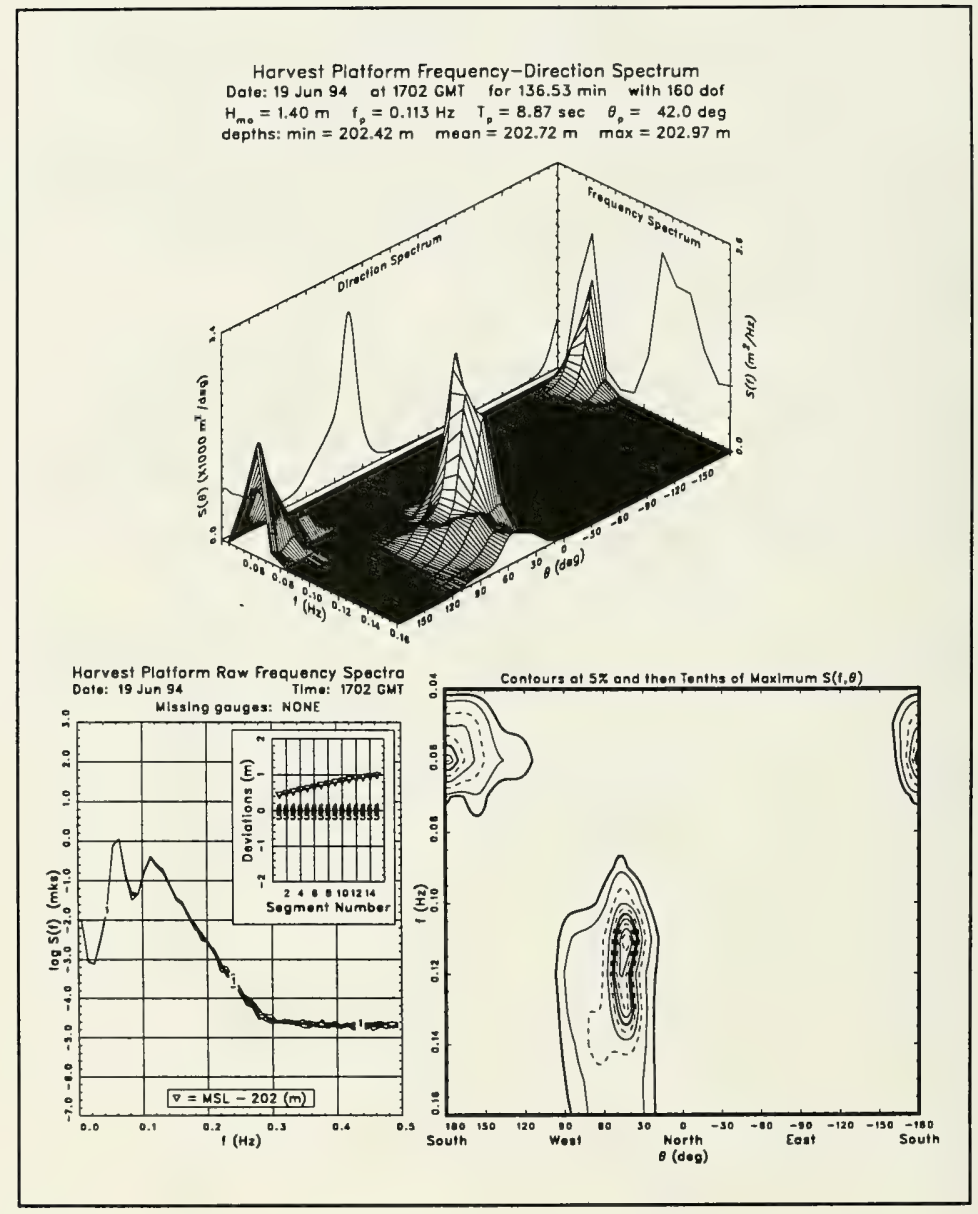

Figure 3. Autospectral intercomparison and frequency-direction spectral estimate

where $\operatorname{Re}[$ ] and $\operatorname{Im}[$ ] are the real and imaginary parts, respectively, of the entity contained in square brackets.

Signals from multiple pairs of gauges having redundant lag (or spatial separation) vectors in a uniform wave field are expected to have identical cross spectra. In the Harvest Platform array there are several such sets of pairs as can be seen in Figure 2. In terms of coherences and phases, one would expect

$$
\Gamma_{14}\left(f_{n}\right)=\Gamma_{23}\left(f_{n}\right)=\Gamma_{56}\left(f_{n}\right) \quad \phi_{14}\left(f_{n}\right)=\phi_{23}\left(f_{n}\right)=\phi_{56}\left(f_{n}\right)
$$


as well as

$$
\Gamma_{12}\left(f_{n}\right)=\Gamma_{43}\left(f_{n}\right) \quad \phi_{12}\left(f_{n}\right)=\phi_{43}\left(f_{n}\right)
$$

and

$$
\Gamma_{15}\left(f_{n}\right)=\Gamma_{46}\left(f_{n}\right) \quad \phi_{15}\left(f_{n}\right)=\phi_{46}\left(f_{n}\right)
$$

Figure 4 is an example of coherence and phase comparisons, showing graphs of the functions named in Equations 4, 5, and 6 (upper, middle, and lower sets of graphs in Figure 4, respectively). This type of error checking is useful for isolating cases where a data point is dropped during telephone transmission from the data buffer, resulting in an apparent temporal shift of data from one gauge relative to data from the other gauges. Such a shift causes a significant phase error in cross spectra, and is readily apparent in a graphic display like Figure 4.

The combined effects of intercomparing frequency autospectra and coherence and phase functions for the pressure gauge array on Harvest Platform provide clear indications of faulty or suspect data. When such conditions are detected in a collection, frequency-direction spectra are not computed. Such rigorous examination of the data ensures that only high-quality time series are used in directional estimation.

\section{Frequency-Direction Spectra}

Estimates of frequency-direction spectra are made using the iterative maximum likelihood estimator (IMLE) developed by Pawka (1983). Estimates are made by iterative approximations of directional distribution functions $D\left(f_{n}, \theta_{m}\right)$, which are related to corresponding frequency-direction spectra $S\left(f_{n}, \theta_{m}\right)$ by

$$
D\left(f_{n}, \theta_{m}\right)=\frac{S\left(f_{n}, \theta_{m}\right)}{S\left(f_{n}\right)}
$$

where $\theta_{m}$ is a discrete angle indicating the direction from which wave energy arrives, measured counterclockwise from true north (Figure 2), and $s\left(f_{n}\right)$ is the (surface-corrected) frequency spectrum. The direction index $m$ ranges from $m=1$ to $m=M=181$, while direction ranges from $\theta_{1}=-180 \mathrm{deg}$ to $\theta_{181}=180 \mathrm{deg}$ in steps of $d \theta=2 \mathrm{deg}$. The directional distribution function has the property

$$
\sum_{m=1}^{M} D\left(f_{n}, \theta_{m}\right) d \theta=1
$$

which must be satisfied in all estimates. 


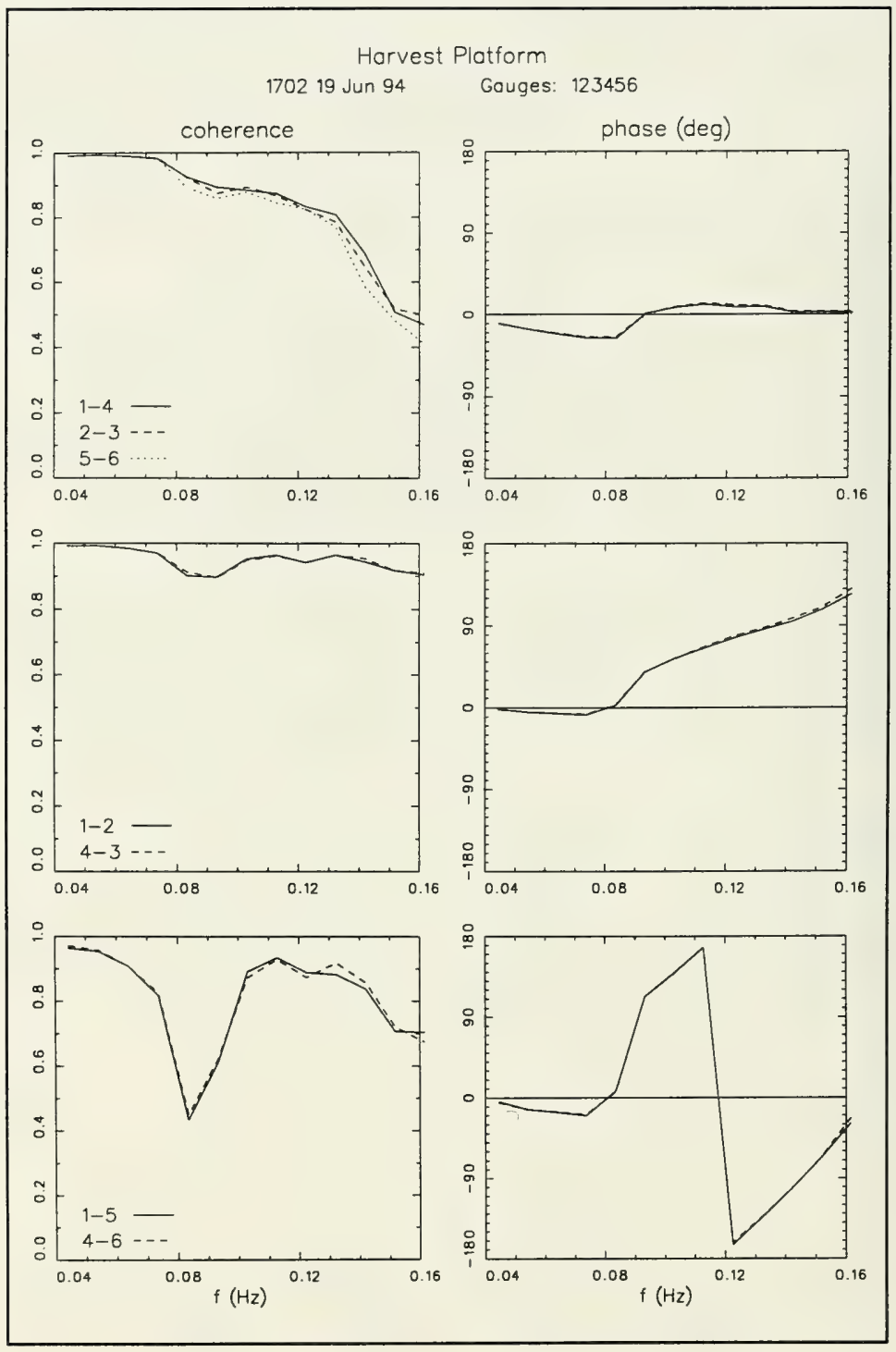

Figure 4. Sample coherence and phase function comparisons

The lowest order estimate is the maximum likelihood estimate described by Davis and Regier (1977), which takes the form 


$$
D_{0}\left(f_{n}, \theta_{m}\right)=\frac{a_{0}}{d \theta \sum_{i=1}^{I} \sum_{j=1}^{I} M_{i j}^{-1}\left(f_{n}\right) e^{i \vec{k}_{n}\left(\theta_{m}\right) \cdot\left(\vec{x}_{i}-\vec{x}_{j}\right)}}
$$

where $a_{0}$ is a factor of order 1 that is used to satisfy Equation 8, $I$ is the number of gauges, the $M_{i j}^{-1}\left(f_{n}\right)$ are elements of the inverse of the dimensionless crossspectral matrix defined by Equation $1, \vec{k}_{n}\left(\theta_{m}\right)$ is the wave number vector, and $\overrightarrow{\boldsymbol{x}}_{\boldsymbol{i}}$ and $\overrightarrow{\boldsymbol{x}}_{j}$ are coordinate position vectors of gauges $i$ and $j$, respectively. The wave number vector $\vec{k}_{n}\left(\theta_{m}\right)$ is

$$
\vec{k}_{n}\left(\theta_{m}\right)=k_{n} \cos \theta_{m} \hat{e}_{x}+k_{n} \sin \theta_{m} \hat{e}_{y}
$$

where $\hat{e}_{x}$ and $\hat{e}_{y}$ are spatial coordinate unit vectors in the $x$-and $y$-directions, respectively, and $k_{n}$ is wave number vector magnitude, which is related with gravitational acceleration $g$ to frequency $f_{n}$ and water depth $d$ through the linear wave dispersion relation

$$
4 \pi^{2} f_{n}^{2}=g k_{n} \tanh k_{n} d
$$

As used in this report, horizontal coordinates are such that $x$ increases to the north, and $y$ increases to the west.

An IMLE result is achieved by iterating through several computational steps. At the $r^{\text {th }}$ iteration, an estimate ${ }^{r} M_{i j}\left(f_{n}\right)$ of the observed cross-spectral matrix $M_{i j}\left(f_{n}\right)$ is computed from the previous directional distribution function estimate $D_{r-1}\left(f_{n}, \theta_{m}\right)$ by

$$
{ }^{r} M_{i j}\left(f_{n}\right)=\sum_{m=1}^{M} D_{r-1}\left(f_{n}, \theta_{m}\right) e^{i \vec{k}_{n}\left(\theta_{m}\right) \cdot\left(\vec{x}_{i}-\vec{x}_{j}\right)} d \theta
$$

A new intermediate directional distribution function estimate $D_{r}^{\prime}\left(f_{n}, \theta_{m}\right)$ is computed using the cross-spectral matrix of Equation 12 in the expression

$$
D_{r}^{\prime}\left(f_{n}, \theta_{m}\right)=\frac{a_{r}}{d \theta \sum_{i=1}^{I} \sum_{j=1}^{I}{ }^{\prime} M_{i j}^{-1}\left(f_{n}\right) e^{i \vec{k}_{n}\left(\theta_{m}\right) \cdot\left(\vec{x}_{i}-\vec{x}_{j}\right)}}
$$

where $a_{r}$ is adjusted so that Equation 8 is satisfied for $D_{r}^{\prime}\left(f_{n}, \theta_{m}\right)$. A correction is found for $D_{r}^{\prime}\left(f_{n}, \theta_{m}\right)$ by first computing 


$$
\lambda_{r}\left(f_{n}, \theta_{m}\right)=1-\frac{D_{r}^{\prime}\left(f_{n}, \theta_{m}\right)}{D_{0}\left(f_{n}, \theta_{m}\right)}
$$

and then finding a new directional distribution function estimate $D_{r}\left(f_{n}, \theta_{m}\right)$ from

$$
D_{r}\left(f_{n}, \theta_{m}\right)=D_{r}^{\prime}\left(f_{n}, \theta_{m}\right)\left[1+\frac{\left|\lambda_{r}\left(f_{n}, \theta_{m}\right)\right|^{\beta+1}}{\gamma \lambda_{r}\left(f_{n}, \theta_{m}\right)}\right]
$$

The parameters $\beta$ and $\gamma$ in Equation 15 control the rate of convergence of the estimator. As used by Pawka (1983), the values $\beta=1$ and $\gamma=5$ were used for all estimates discussed in this report.

In each iterative loop, a convergence check $\epsilon_{r}$ is computed as the sum of the squares of the magnitudes of the differences of elements of the estimated cross spectrum of Equation 12 and the measured cross spectrum of Equation 1. This takes the form

$$
\epsilon_{r}=\sum_{i=1}^{I} \sum_{j=1}^{I}\left|{ }^{r} M_{i j}\left(f_{n}\right)-M_{i j}\left(f_{n}\right)\right|^{2}
$$

Iteration continues as long as $\epsilon_{\mathrm{r}}$ decreases between successive iterations, or until an upper limit $R$ of iterations has been completed. In computations reported herein, $R=30$.

Equations 9 to 16 form the basis of the IMLE technique. For the iteration $r$ that satisfies the convergence check, the frequency-direction spectrum at frequency $f_{n}$ is formed from

$$
S\left(f_{n}, \theta_{m}\right)=S\left(f_{n}\right) D_{r}\left(f_{n}, \theta_{m}\right)
$$

The complete frequency-direction spectrum is formed when Equations 9 through 17 are evaluated for all frequencies.

An example of such a spectrum is illustrated in Figure 3. The upper graph is a three-dimensional plot of $S\left(f_{n}, \theta_{m}\right)$, and the lower right graph is a contour plot of the spectrum. The right panel in the three-dimensional plot is a linear graph of the discrete frequency spectrum $S\left(f_{n}\right)$, which is related to the frequency-direction spectrum through Equations 7 and 8 by

$$
S\left(f_{n}\right)=\sum_{m=1}^{M} S\left(f_{n}, \theta_{m}\right) d \theta
$$


The left panel in the three-dimension plot is a linear graph of the direction spectrum $S\left(\theta_{m}\right)$, which is the directional analog of the frequency spectrum. The direction spectrum is defined by

$$
S\left(\theta_{m}\right)=\sum_{n=1}^{N} S\left(f_{n}, \theta_{m}\right) d f
$$

Because $S\left(\theta_{m}\right)$ represents total wave energy in each direction bin, it is a particularly useful function from which to derive direction-sensitive characterizing parameters for a given frequency-direction spectrum as a whole. A set of such characterizing parameters is defined in Chapter 4. 


\section{Characterizing Parameters}

To effect a summary description of the Harvest Platform database, frequencydirection spectra are characterized with a set of parameters. These descriptors are called bulk parameters because they are derived from extremal or integral properties of spectra, and so represent only part of the frequently more complicated directional structure of the wind wave field. A more exhaustive treatment of directional spectral structure at Harvest Platform is given by Long (1995b). For the purposes of the present report, nine parameters are used. These parameters are: characteristic wave height, peak frequency, two measures of characteristic direction, two measures of directional spread, two measures of asymmetry of directionally distributed wave energy, and a measure of kurtosis of directional distributions. This chapter contains the mathematical definitions of these parameters.

\section{Wave Height, Peak Frequency, and Peak Direction}

Characteristic wave height $H_{m o}$ is defined using the conventional definition of four times the standard deviation of sea surface displacement. $H_{m o}$ can be defined in terms of the full frequency-direction spectrum, the frequency spectrum defined by Equation 18, or the direction spectrum defined by Equation 19. A definition that relates all of these entities is

$$
\frac{H_{m o}^{2}}{16}=\sum_{m=1}^{M} \sum_{n=1}^{N} S\left(f_{n}, \theta_{m}\right) d f d \theta=\sum_{n=1}^{N} S\left(f_{n}\right) d f=\sum_{m=1}^{M} S\left(\theta_{m}\right) d \theta
$$

It should be noted that $H_{m o}$ reported herein is lower than what would be found in conventional analysis because directional computations were truncated at $0.16 \mathrm{~Hz}$ instead of the nominal $0.3-\mathrm{Hz}$ limit for wind waves. Consequently, contributions to $H_{m o}$ from high-frequency parts of wind wave spectra are not represented.

Peak frequency $f_{p}$ is defined as the discrete frequency at which the frequency spectrum $S\left(f_{n}\right)$ is maximum. This definition is conventional, in that it is the usual characteristic frequency defined for nondirectional gauges. For convenience, Appendix A lists both $f_{p}$ and its inverse, peak period $T_{p}\left(=1 / f_{p}\right)$. 
Peak direction $\theta_{p}$ is defined as the direction of maximum variance density in the directional distribution associated with the peak frequency. In symbols, $\theta_{p}$ is the discrete direction at which $S\left(f_{p}, \theta_{m}\right)$ is a maximum. It is interpreted as the direction of the most energetic waves at the frequency containing the greatest overall energy.

\section{Circular Moment Parameters}

Kuik, van Vledder, and Holthuijsen (1988) proposed a useful set of parameters that define mean wave direction, directional spread, skewness, and kurtosis based on circular moments of directional distribution functions. Though derived for directional distributions at individual frequencies, the definitions can be applied to any directional distribution function. For the purposes of characterizing a frequency-direction spectrum as a whole, the direction spectrum $S\left(\theta_{m}\right)$, as defined by Equation 19, is used herein because it represents total wave energy in any given direction arc.

To define a directional distribution function (one that integrates to unit area) from the direction spectrum, $S\left(\theta_{m}\right)$ must be normalized by its own area. By

Equation 20, this area is identically $\frac{1}{16} H_{m o}^{2}$, so the appropriate directional distribution function is

$$
D\left(\theta_{m}\right)=\frac{16}{H_{m o}^{2}} S\left(\theta_{m}\right) \quad m=1,2, \ldots, M
$$

Circular moments in terms of $D\left(\theta_{m}\right)$ adapted from definitions by Kuik, van Vledder, and Holthuijsen (1988) are

$$
\begin{aligned}
& m_{1}=\sum_{m=1}^{M} \cos \left(\theta_{m}-\theta_{0}\right) D\left(\theta_{m}\right) d \theta \\
& n_{1}=\sum_{m=1}^{M} \sin \left(\theta_{m}-\theta_{0}\right) D\left(\theta_{m}\right) d \theta \\
& m_{2}=\sum_{m=1}^{M} \cos \left(2 \theta_{m}-2 \theta_{0}\right) D\left(\theta_{m}\right) d \theta \\
& n_{2}=\sum_{m=1}^{M} \sin \left(2 \theta_{m}-2 \theta_{0}\right) D\left(\theta_{m}\right) d \theta
\end{aligned}
$$

where $\theta_{0}$ is the mean direction defined by requiring $n_{1}=0$. With this constraint, Equation 23 can be solved to find 


$$
\theta_{0}=\tan ^{-1}\left[\frac{\sum_{m=1}^{M} D\left(\theta_{m}\right) \sin \theta_{m} d \theta}{\sum_{m=1}^{M} D\left(\theta_{m}\right) \cos \theta_{m} d \theta}\right]
$$

With $\theta_{0}$ determined by Equation 26, moments $m_{1}, m_{2}$, and $n_{2}$ can be computed from Equations 22, 24, and 25, respectively.

Kuik, van Vledder, and Holthuijsen (1988) define a measure of directional spread (herein called circular width) $\sigma$ as

$$
\sigma=\left(2-2 \dot{m}_{1}\right)^{1 / 2}
$$

a measure of asymmetry of a directional distribution (circular skewness) $\gamma$ as

$$
\gamma=\frac{-n_{2}}{\left(\frac{1}{2}-\frac{1}{2} m_{2}\right)^{3 / 2}}
$$

and a measure of the flatness of a directional distribution (circular kurtosis) $\delta$ as

$$
\delta=\frac{6-8 m_{1}+2 m_{2}}{\left(2-2 m_{1}\right)^{2}}
$$

\section{Quartile Parameters}

Two parameters that are modestly more intuitive than the corresponding circular parameters, and are also useful for characterizing spread and asymmetry in directional distribution function are the quartile spread $\Delta \theta$ and quartile asymmetry $A$ used by Long and Oltman-Shay (1991). The concept is based on the fact that any directional distribution function integrates to unity such that an integral from the direction of minimum energy $\theta_{m_{\min }}$ (where $m_{\min }$ is the discrete direction index at which minimum energy occurs) to any arbitrary angle creates a function $I\left(\theta_{m}-\theta_{m_{m i n}}\right)$ that increases monotonically from zero to an upper limit of unity. The directions at which this integral (interpolated as necessary from discrete data) has the values $\frac{1}{4}, \frac{1}{2}$, and $\frac{3}{4}$ are the first quartile, median, and third quartile directions of the directional distribution, respectively. Differences among these directions then provide information about the spread and asymmetry of the distribution. 
Using $D\left(\theta_{m}\right)$ as a representative directional distribution function, the integral function is

$$
I\left(\theta_{m}-\theta_{m_{\min }}\right)=\sum_{l=m_{\min }}^{m} D\left(\theta_{l}\right) d \theta
$$

where the cyclic nature of the distribution function is employed if necessary. Quartile directions satisfy

$$
\begin{aligned}
& I\left(\theta_{25 \%}-\theta_{m_{\min }}\right)=0.25 \\
& I\left(\theta_{50 \%}-\theta_{m_{\min }}\right)=0.50
\end{aligned}
$$

and

$$
I\left(\theta_{75 \%}-\theta_{m_{\min }}\right)=0.75
$$

A measure of directional spread $\Delta \theta$ is the span of the two middle quartiles

$$
\Delta \theta=\theta_{75 \%}-\theta_{25 \%}
$$

and has the specific interpretation that it is the arc subtending the central 50 percent of the energy distribution.

A measure of asymmetry of a distribution is the ratio of the directional width of the third quartile to that of the second quartile. By taking the natural logarithm of this ratio, a symmetric distribution has an asymmetry parameter $A$ near zero, and that for a skewed distribution acquires a positive or negative sign if the skewness is toward larger or smaller angles, respectively. The asymmetry parameter is thus defined as

$$
A=\ln \left[\frac{\theta_{75 \%}-\theta_{50 \%}}{\theta_{50 \%}-\theta_{25 \%}}\right]
$$

\section{Summary of Parameters}

The nine bulk parameters $\left(H_{m o}, f_{p}, \theta_{p}, \theta_{0}, \sigma, \gamma, \delta, \Delta \theta\right.$, and $\left.A\right)$ defined here are useful for classifying general wind wave energy distributions. For reference as an index of processed data from the 1994 collection year, these parameters are listed in Appendix A, and plotted as time series in Appendix B. Graphs in Appendix B provide an overview of the directional wave climate at Harvest Plat- 
form, and specific parametric values can be determined from the listing in Appen$\operatorname{dix} A$. An evaluation of the accuracy of these parameters, relationships among these parameters, and examples of frequency-direction spectra classified by ranges of these parameters are given by Long (1995b). 


\section{Accessing Spectra}

Frequency-direction spectra computed from Harvest Platform data are currently stored on electro-optical media in binary, unformatted form, and so are not "on line" in the sense of common data networks. Nonetheless, an individual interested in obtaining these spectra can readily do so by communicating with the FRF via:

$\begin{array}{ll}\text { Surface mail } & \text { Chief, Field Research Facility } \\ & \text { 1261 Duck Road } \\ & \text { Kitty Hawk, NC 27949-4472 }\end{array}$

Telephone $\quad$ (919) 261-3511

FAX (919) 261-4432

or any of the following internet addresses:

C.Long@duck.wes.army.mil
C.Baron@duck.wes.army.mil
W.Birkemeier@duck.wes.army.mil

On request, all or part of the spectral database can be converted to 80-column ASCII format and copied either to portable magnetic tape media or to an anonymous file transfer protocol ( $\mathrm{ftp}$ ) account that is accessible through common computer networks. Data will be in the form of a set of files with one spectral estimate per file. Files will be named HPyymmddhhmm.ASC, where yymmdd represents year, month, and day, and hhmm represents hour and minute (GMT) of a collection start time from which a spectrum is estimated. For convenience, dates and times of parameter listings in Appendix A are in the yymmdd and hhmm mnemonic forms.

On receipt by a user, spectral data files can be read using the format statements shown in the sample FORTRAN program listed in Appendix C. The header of the FORTRAN program listing identifies all the variables contained in a data file. For reference, Appendix D is a listing of a sample data file, and shows locations of variables within the file. 


\section{Summary}

This is the second of a series of reports describing results from a high-resolution directional wave gauge installed on the Texaco Oil Company Harvest Platform. The purpose of this gauge is long-term monitoring of the directional wind wave climate at a deepwater site that can be used to represent open ocean conditions for waves approaching the coast of southern California. This report indexes parameters of and describes a means of access to 2,320 frequency-direction spectral observations made during calendar year 1994.

The primary intent of this report is to publicize these observations so that they can be used by researchers interested in seaward boundary conditions in coastal wave propagation models, studies of ocean wave evolution, comparison studies with locally deployed low-resolution directional wave gauges, and ground truth in remote sensing research. Improved knowledge resulting from such studies will enhance abilities to model the physics of open ocean wave processes, and the consequent nearshore wave climate required in coastal engineering computations as such waves propagate landward. 


\section{References}

Bendat, J. S., and Piersol, A. G. (1971). Random data: Analysis and measurement procedures. Wiley-Interscience, New York.

Davis, R. E., and Regier, L. A. (1977). "Methods for estimating directional wave spectra from multi-element arrays," Journal of Marine Research 35, 453-77.

Dean, R. G., and Dalrymple, R. A. (1984). Water wave mechanics for engineers and scientists. Prentice-Hall, Englewood Cliffs, NJ.

Kuik, A. J., van Vledder, G. Ph., and Holthuijsen, L. H. (1988). "A method for the routine analysis of pitch-and-roll buoy wave data," Journal of Physical Oceanography 18, 1020-34.

Long, C. E. (1995a). "1993 annual index of wind wave directional spectra measured at Harvest Platform," Miscellaneous Paper CERC-95-6, U.S. Army Engineer Waterways Experiment Station, Vicksburg, MS.

Long, C. E. (1995b). "Directional wind wave characteristics at Harvest Platform," Technical Report CERC-95-4, U.S. Army Engineer Waterways Experiment Station, Vicksburg, MS.

Long, C. E., and Oltman-Shay, J. M. (1991). "Directional characteristics of waves in shallow water," Technical Report CERC-91-1, U.S. Army Engineer Waterways Experiment Station, Vicksburg, MS.

Monthly reports. Coastal Data Information Program, Scripps Institution of Oceanography, University of California, San Diego, CA.

Pawka, S. S. (1983). "Island shadows in wave directional spectra," Journal of Geophysical Research 88, 2579-91.

Welch, P. D. (1967). "The use of fast Fourier transform for the estimation of power spectra: A method based on time averaging over short, modified periodograms," IEEE Transactions on Audio and Electroacoustics AU-15, 70-3. 



\section{Appendix A \\ Table of Collection Times and Bulk Parameters}

\begin{tabular}{|c|c|c|c|c|c|c|c|c|c|c|c|}
\hline \multicolumn{12}{|c|}{$\begin{array}{l}\text { Table A1 } \\
\text { Collection Times and Bulk Parameters }\end{array}$} \\
\hline Date & $\begin{array}{l}\text { Time } \\
\text { GMT }\end{array}$ & $\begin{array}{l}H_{m o} \\
\mathrm{~m}\end{array}$ & $\begin{array}{l}f_{p} \\
\mathrm{~Hz}\end{array}$ & $\begin{array}{l}T_{p} \\
\text { sec }\end{array}$ & $\begin{array}{l}\theta_{p} \\
\text { deg }\end{array}$ & $\begin{array}{l}\theta_{0} \\
\text { deg }\end{array}$ & $\sigma$ & $Y$ & $\delta$ & $\begin{array}{l}\Delta \theta \\
\text { deg }\end{array}$ & A \\
\hline 940101 & 0159 & 1.92 & 0.074 & 13.6 & 58 & 78 & 0.56 & 0.64 & 4.05 & 43 & 0.32 \\
\hline 940101 & 0459 & 1.97 & 0.074 & 13.6 & 56 & 75 & 0.55 & 0.62 & 4.32 & 40 & 0.21 \\
\hline 940101 & 0759 & 1.91 & 0.074 & 13.6 & 60 & 74 & 0.54 & 0.72 & 4.42 & 38 & 0.49 \\
\hline 940101 & 1059 & 1.84 & 0.083 & 12.0 & 62 & 78 & 0.52 & 0.65 & 4.81 & 36 & 0.38 \\
\hline 940101 & 1659 & 1.70 & 0.074 & 13.6 & 58 & 78 & 0.56 & 0.50 & 4.06 & 43 & 0.18 \\
\hline 940101 & 1959 & 1.55 & 0.083 & 12.0 & 56 & 75 & 0.59 & 0.64 & 4.34 & 42 & 0.14 \\
\hline 940102 & 0159 & 1.62 & 0.083 & 12.0 & 58 & 74 & 0.53 & 0.73 & 5.13 & 34 & 0.05 \\
\hline 940102 & 0459 & 1.81 & 0.083 & 12.0 & 58 & 75 & 0.51 & 0.71 & 5.08 & 35 & 0.10 \\
\hline 940103 & 0054 & 2.48 & 0.064 & 15.6 & 72 & 75 & 0.44 & 0.34 & 6.35 & 28 & 0.15 \\
\hline 940103 & 0459 & 3.01 & 0.064 & 15.6 & 70 & 71 & 0.37 & 0.59 & 10.36 & 15 & 0.24 \\
\hline 940103 & 0759 & 3.04 & 0.064 & 15.6 & 68 & 71 & 0.37 & 0.35 & 10.17 & 15 & 0.30 \\
\hline 940103 & 1100 & 2.99 & 0.074 & 13.6 & 68 & 72 & 0.41 & 0.83 & 8.47 & 18 & 0.24 \\
\hline 940103 & 1400 & 2.87 & 0.064 & 15.6 & 72 & 74 & 0.42 & 0.53 & 7.43 & 22 & 0.13 \\
\hline 940103 & 1704 & 2.78 & 0.064 & 15.6 & 70 & 72 & 0.42 & 0.59 & 8.14 & 20 & 0.23 \\
\hline 940103 & 2000 & 2.90 & 0.064 & 15.6 & 68 & 70 & 0.40 & 0.71 & 8.53 & 20 & 0.19 \\
\hline 940104 & 0802 & 2.84 & 0.064 & 15.6 & 74 & 72 & 0.45 & 0.54 & 6.78 & 25 & -0.14 \\
\hline 940104 & 1100 & 2.55 & 0.064 & 15.6 & 72 & 72 & 0.48 & 0.58 & 5.82 & 28 & -0.12 \\
\hline 940104 & 1358 & 2.48 & 0.064 & 15.6 & 72 & 74 & 0.47 & 0.51 & 5.57 & 28 & 0.09 \\
\hline 940104 & 1713 & 2.42 & 0.064 & 15.6 & 74 & 73 & 0.48 & 0.65 & 5.50 & 29 & 0.05 \\
\hline 940104 & 1956 & 2.39 & 0.074 & 13.6 & 60 & 73 & 0.47 & 0.66 & 5.66 & 28 & 0.26 \\
\hline 940105 & 0158 & 2.41 & 0.074 & 13.6 & 54 & 70 & 0.53 & 0.61 & 4.76 & 36 & 0.33 \\
\hline 940105 & 0501 & 2.50 & 0.074 & 13.6 & 56 & 63 & 0.52 & 0.39 & 4.69 & 33 & 0.28 \\
\hline 940105 & 0758 & 2.59 & 0.064 & 15.6 & 80 & 62 & 0.55 & 0.26 & 4.02 & 40 & -0.17 \\
\hline 940105 & 1100 & 2.41 & 0.074 & 13.6 & 58 & 64 & 0.54 & 0.46 & 4.23 & 38 & -0.01 \\
\hline 940105 & 1359 & 2.58 & 0.074 & 13.6 & 56 & 62 & 0.51 & 0.51 & 4.30 & 36 & 0.23 \\
\hline 940105 & 1951 & 3.62 & 0.123 & 8.2 & 56 & 55 & 0.46 & 0.16 & 4.27 & 34 & -0.09 \\
\hline 940106 & 0200 & 2.89 & 0.113 & 8.9 & 54 & 57 & 0.47 & 0.59 & 5.27 & 31 & 0.12 \\
\hline 940106 & 0500 & 2.45 & 0.113 & 8.9 & 48 & 57 & 0.46 & 0.77 & 5.78 & 31 & 0.32 \\
\hline 940106 & 1400 & 2.23 & 0.083 & 12.0 & 56 & 58 & 0.42 & 0.70 & 7.11 & 27 & 0.05 \\
\hline
\end{tabular}




\begin{tabular}{|c|c|c|c|c|c|c|c|c|c|c|c|}
\hline \multicolumn{12}{|c|}{ Table A1 (Continued) } \\
\hline Date & $\begin{array}{l}\text { Time } \\
\text { GMT }\end{array}$ & $\begin{array}{l}H_{m o} \\
\mathrm{~m}\end{array}$ & $\begin{array}{l}f_{p} \\
\mathrm{~Hz}\end{array}$ & $\begin{array}{l}T_{p} \\
\sec \end{array}$ & $\begin{array}{l}\theta_{p} \\
\text { deg }\end{array}$ & $\begin{array}{l}\theta_{0} \\
\operatorname{deg}\end{array}$ & $\sigma$ & $\mathrm{Y}$ & $\delta$ & $\begin{array}{l}\Delta \theta \\
\operatorname{deg}\end{array}$ & $A$ \\
\hline 940106 & 1704 & 2.28 & 0.083 & 12.0 & 50 & 59 & 0.44 & 0.72 & 6.50 & 31 & 0.24 \\
\hline 940107 & 0804 & 1.68 & 0.093 & 10.7 & 50 & 51 & 0.49 & 1.08 & 6.78 & 28 & 0.01 \\
\hline 940107 & 1057 & 1.55 & 0.093 & 10.7 & 50 & 52 & 0.48 & 1.44 & 7.00 & 22 & 0.03 \\
\hline 940107 & 1401 & 1.38 & 0.093 & 10.7 & 52 & 55 & 0.50 & 1.40 & 6.40 & 25 & -0.01 \\
\hline 940107 & 1707 & 1.39 & 0.103 & 9.7 & 54 & 55 & 0.51 & 1.12 & 5.63 & 29 & -0.03 \\
\hline 940107 & 1958 & 1.27 & 0.093 & 10.7 & 48 & 55 & 0.51 & 1.34 & 6.10 & 28 & 0.39 \\
\hline 940108 & 0503 & 1.01 & 0.103 & 9.7 & 56 & 61 & 0.60 & 1.49 & 5.15 & 30 & 0.24 \\
\hline 940108 & 0801 & 0.87 & 0.103 & 9.7 & 54 & 65 & 0.67 & 1.22 & 4.21 & 41 & 0.61 \\
\hline 940108 & 1403 & 0.88 & 0.113 & 8.9 & 56 & 64 & 0.63 & 1.87 & 4.96 & 31 & 0.37 \\
\hline 940108 & 1703 & 0.80 & 0.074 & 13.6 & 58 & 65 & 0.67 & 1.33 & 3.89 & 40 & 0.52 \\
\hline 940108 & 2001 & 0.76 & 0.074 & 13.6 & 54 & 71 & 0.73 & 1.09 & 3.26 & 49 & 0.57 \\
\hline 940109 & 0203 & 1.05 & 0.162 & 6.2 & 26 & 52 & 0.69 & 0.98 & 4.12 & 46 & 0.07 \\
\hline 940109 & 0503 & 1.09 & 0.162 & 6.2 & 50 & 56 & 0.59 & 1.08 & 4.53 & 40 & 0.31 \\
\hline 940109 & 0803 & 1.27 & 0.162 & 6.2 & 54 & 59 & 0.52 & 1.14 & 6.36 & 27 & 0.23 \\
\hline 940109 & 1101 & 1.79 & 0.064 & 15.6 & 58 & 59 & 0.49 & 1.01 & 7.02 & 20 & -0.02 \\
\hline 940109 & 1353 & 2.67 & 0.074 & 13.6 & 60 & 61 & 0.38 & 0.61 & 10.14 & 15 & 0.02 \\
\hline 940109 & 1704 & 3.02 & 0.064 & 15.6 & 64 & 61 & 0.38 & 0.41 & 8.64 & 19 & -0.12 \\
\hline 940109 & 2003 & 2.99 & 0.074 & 13.6 & 54 & 60 & 0.43 & 0.76 & 8.42 & 22 & 0.11 \\
\hline 940110 & 0159 & 2.92 & 0.074 & 13.6 & 56 & 58 & 0.43 & 0.49 & 7.56 & 23 & 0.91 \\
\hline 940110 & 0459 & 2.81 & 0.074 & 13.6 & 56 & 58 & 0.42 & 0.40 & 7.89 & 22 & 0.06 \\
\hline 940110 & 0759 & 2.76 & 0.074 & 13.6 & 56 & 58 & 0.45 & 0.74 & 7.10 & 25 & 0.14 \\
\hline 940110 & 1056 & 2.42 & 0.083 & 12.0 & 52 & 60 & 0.48 & 0.97 & 6.76 & 27 & 0.47 \\
\hline 940110 & 1645 & 1.96 & 0.083 & 12.0 & 58 & 62 & 0.52 & 1.04 & 5.95 & 28 & 0.29 \\
\hline 940111 & 0159 & 1.64 & 0.093 & 10.7 & 62 & 69 & 0.53 & 1.30 & 5.78 & 28 & 0.32 \\
\hline 940111 & 0459 & 1.54 & 0.093 & 10.7 & 58 & 67 & 0.52 & 1.24 & 5.86 & 28 & 0.45 \\
\hline 940111 & 0759 & 1.51 & 0.093 & 10.7 & 54 & 63 & 0.55 & 1.36 & 5.48 & 28 & 0.50 \\
\hline 940111 & 1111 & 1.41 & 0.103 & 9.7 & 56 & 66 & 0.57 & 1.46 & 5.51 & 30 & 0.40 \\
\hline 940111 & 1351 & 1.48 & 0.103 & 9.7 & 60 & 65 & 0.60 & 1.26 & 4.99 & 31 & 0.29 \\
\hline 940111 & 1722 & 1.46 & 0.152 & 6.6 & 58 & 62 & 0.59 & 1.39 & 5.30 & 31 & 0.12 \\
\hline 940111 & 1959 & 1.53 & 0.152 & 6.6 & 64 & 62 & 0.50 & 1.05 & 6.36 & 27 & -0.18 \\
\hline 940112 & 0159 & 1.55 & 0.064 & 15.6 & 56 & 63 & 0.54 & 1.14 & 5.60 & 32 & 0.08 \\
\hline 940112 & 0459 & 1.49 & 0.064 & 15.6 & 66 & 63 & 0.53 & 1.45 & 6. & 27 & -0.08 \\
\hline 940112 & 0759 & 1.48 & 0.064 & 15.6 & 54 & 61 & 0.58 & 1.53 & 5.81 & 31 & 0.22 \\
\hline 940112 & 1105 & 1.65 & 0.074 & 13.6 & 56 & 64 & 0.53 & 1.66 & 6.87 & 24 & 0.45 \\
\hline 940112 & 1409 & 1.76 & 0.074 & 13.6 & 58 & 63 & 0.48 & 1.48 & 7.84 & 23 & 0.27 \\
\hline 940112 & 1708 & 1.88 & 0.074 & 13.6 & 60 & 63 & 0.49 & 1.45 & 7.61 & 24 & 0.14 \\
\hline 940112 & 2003 & 1.68 & 0.074 & 13.6 & 58 & 61 & 0.51 & 1.75 & 7.23 & 22 & 0.23 \\
\hline 940113 & 0202 & 1.55 & 0.074 & 13.6 & 54 & 59 & 0.55 & 1.43 & 5.79 & 28 & 0.30 \\
\hline 940113 & 0504 & 1.52 & 0.083 & 12.0 & 52 & 60 & 0.58 & 1.55 & 5.79 & 31 & 0.34 \\
\hline 940113 & 0804 & 1.42 & 0.083 & 12.0 & 58 & 61 & 0.57 & 2.05 & 6.53 & 22 & 0.16 \\
\hline 940113 & 1403 & 1.39 & 0.054 & 18.5 & 52 & 63 & 0.62 & 1.78 & 5.35 & 29 & 0.23 \\
\hline 940113 & 1703 & 1.42 & 0.054 & 18.5 & 68 & 65 & 0.57 & 1.72 & 5.88 & 26 & 0.03 \\
\hline 940113 & 2003 & 1.43 & 0.064 & 15.6 & 64 & 66 & 0.60 & 1.65 & 5.41 & 28 & -0.03 \\
\hline 940114 & 0205 & 1.55 & 0.064 & 15.6 & 66 & 68 & 0.48 & 1.43 & 7.60 & 18 & 0.09 \\
\hline 940114 & 0505 & 1.42 & 0.064 & 15.6 & 70 & 69 & 0.54 & 1.31 & 6.11 & 27 & -0.17 \\
\hline 940114 & 0805 & 1.32 & 0.064 & 15.6 & 70 & 72 & 0.54 & 1.51 & 6.34 & 27 & -0.04 \\
\hline 940114 & 1102 & 1.41 & 0.064 & 15.6 & 62 & 69 & 0.56 & 1.31 & 5.87 & 31 & 0.29 \\
\hline 940114 & 1410 & 1.49 & 0.064 & 15.6 & 64 & 68 & 0.53 & 1.25 & 6.19 & 29 & 0.16 \\
\hline 940114 & 1712 & 1.58 & 0.064 & 15.6 & 68 & 68 & 0.46 & 1.28 & 7.37 & 26 & -0.09 \\
\hline 940114 & 2004 & 1.61 & 0.064 & 15.6 & 70 & 70 & 0.49 & 1.33 & 6.60 & 27 & 0.12 \\
\hline 940115 & 0204 & 1.61 & 0.064 & 15.6 & 70 & 73 & 0.49 & 1.04 & 6.71 & 23 & 0.15 \\
\hline
\end{tabular}




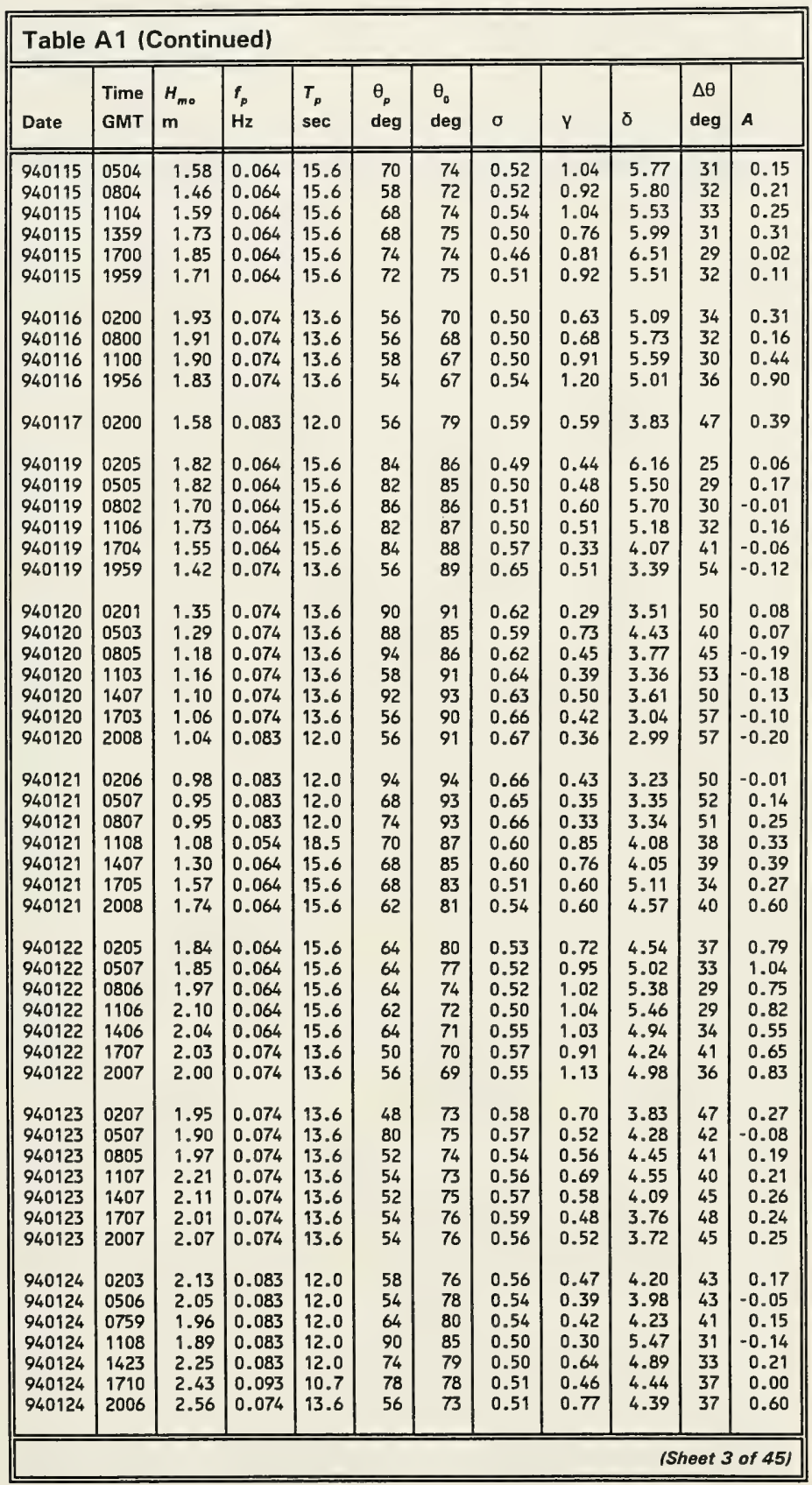




\begin{tabular}{|c|c|c|c|c|c|c|c|c|c|c|c|}
\hline Date & $\begin{array}{l}\text { Time } \\
\text { GMT }\end{array}$ & $\begin{array}{l}H_{m 0} \\
\text { m }\end{array}$ & $\begin{array}{l}f_{p} \\
\mathrm{~Hz}\end{array}$ & $\begin{array}{l}T_{p} \\
\text { sec }\end{array}$ & $\begin{array}{l}\theta_{p} \\
\operatorname{deg}\end{array}$ & $\begin{array}{c}\theta_{0} \\
\operatorname{deg}\end{array}$ & 0 & $Y$ & $\delta$ & $\begin{array}{l}\Delta \theta \\
\operatorname{deg}\end{array}$ & A \\
\hline 940125 & 0209 & 3.24 & 0.083 & 12.0 & 54 & 70 & 0.49 & 0.85 & 5.52 & 32 & 0.16 \\
\hline 940125 & 0510 & 4.18 & .064 & 15.6 & 64 & 66 & 0.44 & 0.81 & 6.73 & 24 & 0.06 \\
\hline 940125 & 1110 & 4.06 & 0.064 & 15.6 & 58 & 64 & 0.43 & 0.98 & 8.02 & 23 & 0.23 \\
\hline 940125 & 1410 & 3.79 & 0.064 & 15.6 & 62 & 66 & 0.43 & 1.08 & 7.51 & 23 & 0.28 \\
\hline 940125 & 1710 & 3.41 & 0.064 & 15.6 & 60 & 66 & 0.45 & 1.21 & 7.48 & 21 & 0.39 \\
\hline 940125 & 2010 & 3.30 & 0.074 & 13.6 & 60 & 67 & 0.46 & 1.17 & 7.10 & 23 & 0.57 \\
\hline 940126 & 0207 & 3.82 & 0.074 & 13.6 & 62 & 64 & 0.40 & 1.04 & 8.41 & 20 & 0.13 \\
\hline 940126 & 0590 & 3.64 & 0.074 & 13.6 & 54 & 63 & 0.42 & 1.23 & 8.15 & 21 & 0.33 \\
\hline 940126 & 0810 & 3.00 & 0.083 & 12.0 & 56 & 64 & 0.46 & 1.04 & 7.24 & 25 & 0.41 \\
\hline 940126 & 1109 & 3.08 & 0.074 & 13.6 & 54 & 63 & 0.42 & 0.98 & 7.84 & 24 & 0.36 \\
\hline 940126 & 1413 & 2.81 & 0.074 & 13.6 & 52 & 63 & 0.44 & 0.93 & 6.80 & 28 & 0.32 \\
\hline 940126 & 1711 & 2.55 & 0.074 & 13.6 & 54 & 64 & 0.45 & 1.08 & 6.74 & 26 & 0.55 \\
\hline 940126 & 2009 & 2.54 & 0.083 & 12.0 & 54 & 63 & 0.45 & 1.21 & 6.65 & 24 & 0.64 \\
\hline 940127 & 0210 & 2.51 & 0.083 & 12.0 & 52 & 63 & 0.47 & 0.77 & 5.48 & 31 & 0.49 \\
\hline 940127 & 0510 & 2.26 & 0.083 & 12.0 & 50 & 63 & 0.49 & 0.71 & 5.11 & 34 & 0.36 \\
\hline 940127 & 0808 & 2.10 & 0.093 & 10.7 & 52 & 63 & 0.48 & 0.74 & 5.99 & 30 & 0.28 \\
\hline 940127 & 1122 & 2.01 & 0.083 & 12.0 & 54 & 67 & 0.49 & 0.57 & 6.09 & 30 & 0.16 \\
\hline 940127 & 1413 & 2.22 & 0.113 & 8.9 & 68 & 65 & 0.44 & 0.60 & 7.46 & 24 & -0.12 \\
\hline 940127 & 1714 & 2.56 & 0.054 & 18.5 & 68 & 65 & 0.40 & 0.41 & 8.46 & 21 & -0.38 \\
\hline 940127 & 2009 & 2.68 & 0.054 & 18.5 & 66 & 63 & 0.44 & 0.50 & 7.40 & 23 & -0.12 \\
\hline 940128 & 0207 & 3.16 & 0.064 & 15.6 & 62 & 63 & 0.40 & 0.72 & 9.55 & 18 & -0.02 \\
\hline 940128 & 0509 & 2.81 & 0.064 & 15.6 & 64 & 62 & 0.39 & 0.55 & 9.42 & 19 & -0.23 \\
\hline 940128 & 0805 & 2.78 & 0.064 & 15.6 & 64 & 62 & 0.39 & 0.83 & 9.76 & 18 & -0.20 \\
\hline 940128 & 1101 & 2.77 & 0.064 & 15.6 & 62 & 60 & 0.41 & 0.84 & 8.69 & 20 & -0.14 \\
\hline 940128 & 1425 & 2.99 & 0.064 & 15.6 & 52 & 58 & 0.39 & 0.89 & 8.97 & 21 & 0.03 \\
\hline 940128 & 1718 & 2.81 & 0.064 & 15.6 & 52 & 58 & 0.42 & 0.92 & 8.33 & 23 & 0.06 \\
\hline 940128 & 2007 & 2.66 & 0.074 & 13.6 & 58 & 59 & 0.41 & 0.90 & 8.55 & 22 & 0.00 \\
\hline 940129 & 0210 & 2.02 & 0.074 & 13.6 & 52 & 58 & 0.47 & 1.70 & 8.51 & 18 & 0.28 \\
\hline 940129 & 0509 & 1.71 & 0.083 & 12.0 & 52 & 60 & 0.51 & 1.88 & 7.71 & 22 & 0.50 \\
\hline 940129 & 0809 & 1.58 & 0.093 & 10.7 & 50 & 60 & 0.55 & 1.82 & 6.59 & 26 & 0.74 \\
\hline 940129 & 1109 & 1.51 & 0.074 & 13.6 & 52 & 61 & 0.51 & 1.77 & 7.32 & 22 & 0.57 \\
\hline 940129 & 1409 & 1.45 & 0.074 & 13.6 & 52 & 69 & 0.51 & 1.66 & 6.88 & 25 & 0.62 \\
\hline 940129 & 1709 & 1.45 & 0.074 & 13.6 & 50 & 58 & 0.54 & 1.95 & 6.56 & 25 & 0.51 \\
\hline 940129 & 2009 & 1.42 & 0.083 & 12.0 & 50 & 59 & 0.53 & 2.11 & 7.26 & 22 & 0.29 \\
\hline 940130 & 0209 & 1.19 & 0.083 & 12.0 & 56 & 65 & 0.60 & 2.01 & 5.68 & 26 & 0.49 \\
\hline 940130 & 0509 & 1.11 & 0.074 & 13.6 & 54 & 63 & 0.59 & 1.91 & 5.54 & 28 & 0.59 \\
\hline 940130 & 0809 & 1.17 & 0.054 & 18.5 & 52 & 61 & 0.59 & 1.87 & 5.81 & 28 & 0.10 \\
\hline 940130 & 1109 & 1.10 & 0.074 & 13.6 & 52 & 61 & 0.61 & 1.95 & 5.42 & 29 & 0.39 \\
\hline 940130 & 1407 & 0.96 & 0.054 & 18.5 & 48 & 70 & 0.73 & 1.52 & 3.64 & 45 & 0.64 \\
\hline 940130 & 1709 & 1.01 & 0.054 & 18.5 & 48 & 71 & 0.68 & 1.42 & 3.85 & 39 & 0.11 \\
\hline 940130 & 2009 & 1.05 & 0.054 & 18.5 & 72 & 73 & 0.68 & 1.56 & 4.12 & 33 & 0.00 \\
\hline 940131 & 0207 & 1.00 & 0.054 & 18.5 & 58 & 76 & 0.68 & 1.38 & 4.07 & 38 & 0.17 \\
\hline 940131 & 0504 & & 0.054 & 18.5 & 76 & 76 & 0.6 & 1.34 & & 37 & 0.14 \\
\hline 940131 & 1115 & 1.55 & 0.064 & 15.6 & 72 & 77 & 0.56 & 1.37 & 5.73 & 25 & 0.33 \\
\hline 940131 & 1422 & 1.74 & 0.064 & 15.6 & 80 & 89 & 0.84 & 2.98 & 4.18 & 37 & 0.61 \\
\hline 940131 & 1713 & 1.86 & 0.064 & 15.6 & 80 & 86 & 0.72 & 1.84 & 5.14 & 31 & 0.45 \\
\hline 940131 & 2009 & 1.91 & 0.064 & 15.6 & 82 & 83 & 0.59 & 0.78 & 5.85 & 28 & 0.04 \\
\hline 940131 & 2309 & 1.98 & 0.064 & 15.6 & 80 & 86 & 0.51 & 0.80 & 5.90 & 27 & 0.46 \\
\hline 940201 & 0209 & 1.89 & 0.064 & 15.6 & 82 & 88 & 0.53 & 0.92 & 5.87 & 29 & 0.40 \\
\hline 940201 & 0509 & 1.83 & 0.074 & 13.6 & 82 & 90 & 0.61 & 1.13 & 4.95 & 37 & 0.33 \\
\hline 940201 & 0807 & 1.94 & 0.064 & 15.6 & 78 & 87 & 0.70 & 1.74 & 4.80 & 38 & 0.62 \\
\hline 940201 & 1122 & 1.83 & 0.074 & 13.6 & 72 & 94 & 0.91 & 2.26 & 3.29 & 60 & 0.72 \\
\hline 940201 & 1430 & 1.87 & 0.074 & 13.6 & 76 & 93 & 0.90 & 2.16 & 3.39 & 58 & 0.91 \\
\hline \multicolumn{12}{|c|}{ (Sheet 4 of 45 ) } \\
\hline
\end{tabular}




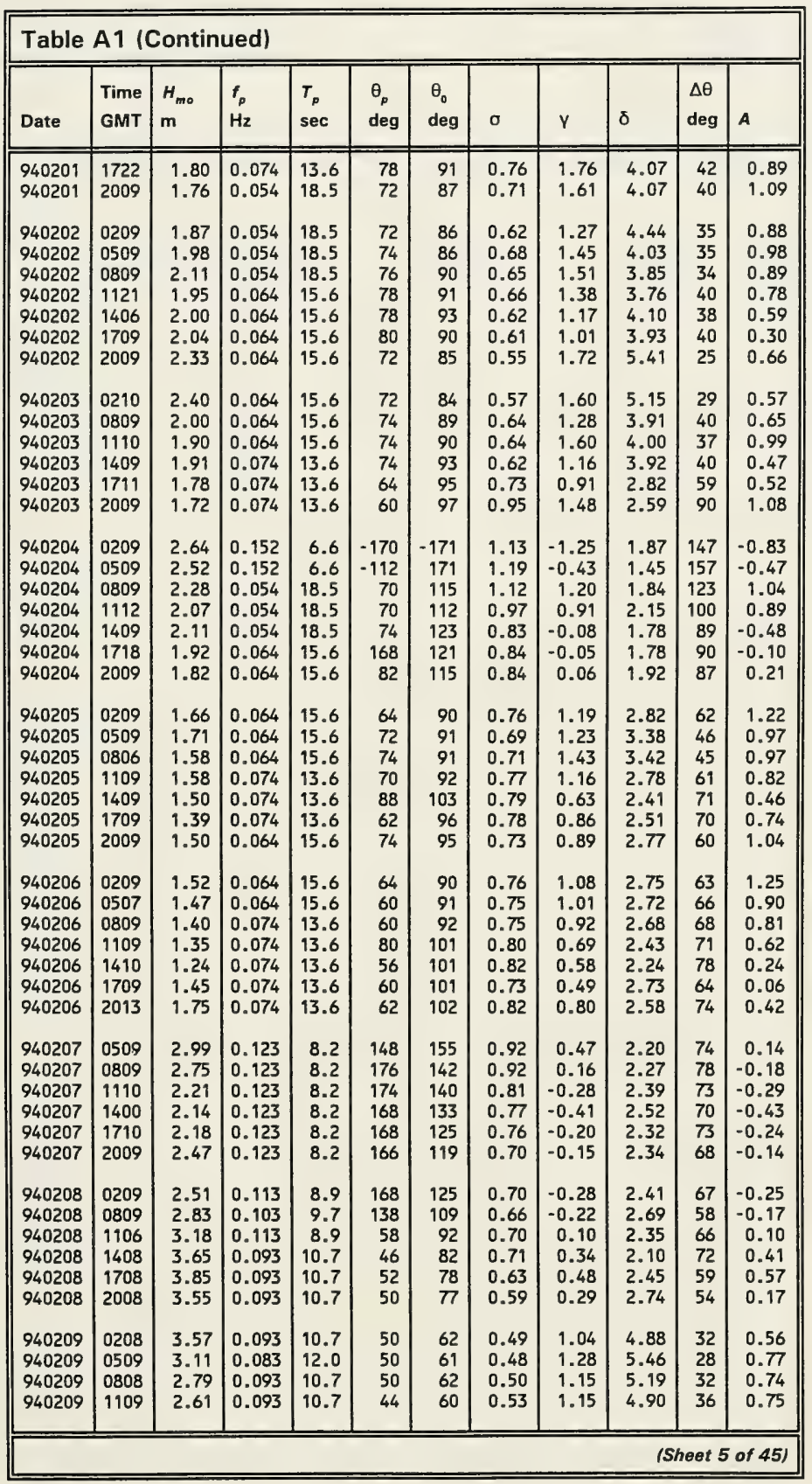




\begin{tabular}{|c|c|c|c|c|c|c|c|c|c|c|c|}
\hline Date & $\begin{array}{l}\text { Time } \\
\text { GMT }\end{array}$ & $\begin{array}{l}H_{m 0} \\
m\end{array}$ & $\begin{array}{l}f_{p} \\
\mathrm{~Hz}\end{array}$ & $\begin{array}{l}T_{p} \\
\mathrm{sec}\end{array}$ & $\begin{array}{l}\theta_{p} \\
\text { deg }\end{array}$ & $\begin{array}{l}\theta_{0} \\
\operatorname{deg}\end{array}$ & $\sigma$ & $Y$ & $\delta$ & $\begin{array}{l}\Delta \theta \\
\operatorname{deg}\end{array}$ & A \\
\hline $\begin{array}{l}940209 \\
940209\end{array}$ & $\begin{array}{l}1409 \\
1715\end{array}$ & $\begin{array}{l}2.64 \\
2.47\end{array}$ & $\begin{array}{l}0.093 \\
0.103\end{array}$ & $\begin{array}{r}10.7 \\
9.7\end{array}$ & $\begin{array}{l}42 \\
46\end{array}$ & $\begin{array}{l}59 \\
58\end{array}$ & $\begin{array}{l}0.52 \\
0.53\end{array}$ & $\begin{array}{l}1.17 \\
1.36\end{array}$ & $\begin{array}{l}5.00 \\
5.11\end{array}$ & $\begin{array}{l}33 \\
32\end{array}$ & $\begin{array}{l}0.40 \\
0.65\end{array}$ \\
\hline 940210 & 0208 & 1.98 & 0.064 & 15.6 & 50 & 61 & 0.56 & 1.11 & 4.94 & 37 & 0.42 \\
\hline 940210 & 0509 & 1.72 & 0.074 & 13.6 & 50 & 63 & 0.58 & 1.10 & 4.63 & 37 & 0.40 \\
\hline 940210 & 0808 & 1.68 & 0.074 & 13.6 & 54 & 66 & 0.59 & 1.06 & 4.31 & 39 & 0.65 \\
\hline 940210 & 1108 & 1.69 & 0.074 & 13.6 & 52 & 63 & 0.58 & 1.39 & 4.90 & 34 & 0.74 \\
\hline 940210 & 1403 & 1.66 & 0.074 & 13.6 & 52 & 65 & 0.57 & 1.04 & 4.66 & 40 & 0.63 \\
\hline 940211 & 0209 & 1.77 & 0.162 & 6.2 & 48 & 57 & 0.56 & 0.85 & 4.37 & 41 & 0.56 \\
\hline 940211 & 0509 & 2.47 & 0.132 & 7.6 & 50 & 51 & 0.50 & 0.85 & 4.72 & 37 & 0.09 \\
\hline 940211 & 0809 & 2.66 & 0.123 & 8.2 & 48 & 55 & 0.42 & 1.27 & 7.03 & 24 & 0.40 \\
\hline 940211 & 1107 & 2.80 & 0.113 & 8.9 & 48 & 55 & 0.41 & 1.27 & 7.96 & 22 & 0.37 \\
\hline 940211 & 1409 & 3.04 & 0.074 & 13.6 & 48 & 53 & 0.40 & 1.27 & 8.35 & 22 & 0.36 \\
\hline 940212 & 1357 & 1.86 & 0.083 & 12.0 & 48 & 56 & 0.63 & 1.70 & 6.78 & 21 & 0.48 \\
\hline 940213 & 0209 & 1.55 & 0.083 & 12.0 & 50 & 61 & 0.55 & 2.21 & 6.70 & 27 & 0.61 \\
\hline 940213 & 0509 & 1.77 & 0.083 & 12.0 & 50 & 58 & 0.53 & 1.71 & 6.94 & 27 & 0.53 \\
\hline 940213 & 1407 & 1.81 & 0.054 & 18.5 & 66 & 63 & 0.55 & 1.56 & 6.57 & 27 & -0.16 \\
\hline 940213 & 1709 & 1.96 & 0.054 & 18.5 & 68 & 65 & 0.49 & 1.55 & 7.58 & 21 & -0.57 \\
\hline 940213 & 2009 & 2.10 & 0.054 & 18.5 & 70 & 68 & 0.45 & 2.20 & 9.76 & 11 & -0.33 \\
\hline 940214 & 0209 & 1.83 & 0.054 & 18.5 & 54 & 65 & 0.55 & 1.76 & 6.31 & 27 & 0.14 \\
\hline 940214 & 0509 & 2.29 & 0.074 & 13.6 & 46 & 62 & 0.51 & 1.39 & 6.18 & 32 & 0.66 \\
\hline 940214 & 0809 & 2.54 & 0.074 & 13.6 & 50 & 59 & 0.46 & 1.41 & 7.73 & 24 & 0.60 \\
\hline 940214 & 2004 & 2.90 & 0.074 & 13.6 & 60 & 61 & 0.38 & 1.57 & 12.20 & 13 & -0.02 \\
\hline 940215 & 0809 & 2.71 & 0.064 & 15.6 & 58 & 60 & 0.42 & 1.60 & 9.80 & 17 & 0.06 \\
\hline 940215 & 1108 & 2.58 & 0.074 & 13.6 & 56 & 61 & 0.45 & 1.38 & 8.69 & 19 & 0.37 \\
\hline 940215 & 1409 & 2.66 & 0.074 & 13.6 & 58 & 61 & 0.44 & 1.52 & 8.86 & 19 & 0.14 \\
\hline 940215 & 2007 & 2.72 & 0.074 & 13.6 & 52 & 58 & 0.46 & 1.63 & 8.00 & 19 & 0.41 \\
\hline 940216 & 0208 & 2.03 & 0.074 & 13.6 & 52 & 61 & 0.51 & 1.70 & 7.36 & 23 & 0.72 \\
\hline 940216 & 1406 & 1.71 & 0.074 & 13.6 & 56 & 66 & 0.53 & 1.54 & 6.38 & 27 & 0.50 \\
\hline 940216 & 1709 & 1.54 & 0.083 & 12.0 & 52 & 65 & 0.58 & 1.55 & 5.23 & 34 & 0.68 \\
\hline 940217 & 0209 & 1.03 & 0.083 & 12.0 & 56 & 94 & 1.16 & 1.98 & 2.17 & 135 & 1.10 \\
\hline 940217 & 0509 & 1.27 & 0.162 & 6.2 & -100 & 173 & 1.25 & -0.17 & 1.39 & 163 & -0.01 \\
\hline 940218 & 0509 & 4.06 & 0.093 & 10.7 & 78 & 86 & 0.51 & 0.23 & 3.92 & 38 & 0.23 \\
\hline 940218 & 0809 & 3.86 & 0.083 & 12.0 & 84 & 83 & 0.51 & 0.36 & 4.31 & 37 & -0.08 \\
\hline 940218 & 1107 & 3.63 & 0.083 & 12.0 & 78 & 82 & 0.53 & 0.39 & 4.08 & 41 & 0.14 \\
\hline 940218 & 1405 & 3.29 & 0.083 & 12.0 & 80 & 82 & 0.55 & 0.48 & 4.07 & 42 & 0.10 \\
\hline 940218 & 1657 & 3.01 & 0.083 & 12.0 & 58 & 78 & 0.59 & 0.77 & 3.79 & 46 & 0.50 \\
\hline 940218 & 2306 & 3.13 & 0.083 & 12.0 & 54 & 72 & 0.54 & 0.70 & 4.20 & 40 & 0.36 \\
\hline 940219 & 0208 & 3.20 & 0.083 & 12.0 & 54 & 72 & 0.52 & 0.88 & 4.83 & 38 & 0.44 \\
\hline 940219 & 0505 & 2.96 & 0.083 & 12.0 & 58 & 74 & 0.53 & 0.82 & 4.6 & 38 & 0.48 \\
\hline 940219 & 0810 & 2.89 & 0.083 & 12.0 & 54 & 70 & 0.53 & 1.16 & 5.00 & 36 & 0.68 \\
\hline 940219 & 1110 & 2.66 & 0.083 & 12.0 & 56 & 70 & 0.53 & 1.09 & 4.95 & 36 & 0.65 \\
\hline 940219 & 1406 & 2.32 & 0.083 & 12.0 & 54 & 72 & 0.60 & 1.10 & 4.13 & 43 & 0.76 \\
\hline 940219 & 1710 & 2.09 & 0.083 & 12.0 & 54 & 73 & 0.63 & 1.35 & 4.17 & 44 & 0.93 \\
\hline 940219 & 2010 & 2.15 & 0.093 & 10.7 & 52 & 75 & 0.61 & 1.14 & 4.04 & 45 & 0.50 \\
\hline 940219 & 2310 & 2.60 & 0.093 & 10.7 & 56 & 88 & 0.68 & 0.70 & 2.75 & 59 & 0.36 \\
\hline 940220 & 0210 & 2.48 & 0.142 & 7.0 & 58 & 92 & 0.72 & 0.58 & 2.55 & 64 & 0.37 \\
\hline 940220 & 0510 & 2.33 & 0.132 & 7.6 & 52 & 88 & 0.71 & 0.46 & 2.47 & 65 & 0.18 \\
\hline 940220 & 0810 & 2.08 & 0.093 & 10.7 & 52 & 83 & 0.72 & 0.61 & 2.72 & 62 & 0.38 \\
\hline 940220 & 1110 & 2.05 & 0.093 & 10.7 & 54 & 76 & 0.70 & 0.97 & 3.01 & 55 & 0.59 \\
\hline
\end{tabular}




\begin{tabular}{|c|c|c|c|c|c|c|c|c|c|c|c|}
\hline \multicolumn{12}{|c|}{ Table A1 (Continued) } \\
\hline Date & $\begin{array}{l}\text { Time } \\
\text { GMT }\end{array}$ & $\begin{array}{l}H_{m 0} \\
m\end{array}$ & $\begin{array}{l}f_{p} \\
H_{2}\end{array}$ & $\begin{array}{l}T_{p} \\
\text { sec }\end{array}$ & $\begin{array}{l}\theta_{p} \\
\text { deg }\end{array}$ & $\begin{array}{l}\theta_{0} \\
\text { deg }\end{array}$ & $\sigma$ & $Y$ & 0 & $\begin{array}{l}\Delta \theta \\
\operatorname{deg}\end{array}$ & $A$ \\
\hline 940220 & 1410 & 2.24 & 0.093 & 10.7 & 54 & 76 & 0.64 & 0.87 & 3.32 & 48 & 0.17 \\
\hline 940220 & 1715 & 2.27 & 0.103 & 9.7 & 56 & 73 & 0.60 & 0.86 & 3.73 & 44 & 0.38 \\
\hline 940220 & 2010 & 2.53 & 0.093 & 10.7 & 58 & 68 & 0.57 & 0.88 & 4.16 & 40 & 0.34 \\
\hline 940221 & 0210 & 2.47 & 0.093 & 10.7 & 56 & 68 & 0.59 & 0.81 & 4.00 & 43 & 0.40 \\
\hline 940221 & 0507 & 2.39 & 0.083 & 12.0 & 58 & 67 & 0.54 & 1.04 & 4.70 & 34 & 0.54 \\
\hline 940221 & 0810 & 2.08 & 0.093 & 10.7 & 50 & 67 & 0.60 & 1.33 & 4.60 & 40 & 0.68 \\
\hline 940221 & 1110 & 2.21 & 0.093 & 10.7 & 50 & 64 & 0.54 & 1.46 & 5.35 & 34 & 0.70 \\
\hline 940221 & 1410 & 2.16 & 0.093 & 10.7 & 52 & 62 & 0.53 & 1.34 & 5.30 & 33 & 0.50 \\
\hline 940221 & 1710 & 2.08 & 0.093 & 10.7 & 54 & 64 & 0.57 & 1.09 & 4.42 & 40 & 0.47 \\
\hline 940221 & 2010 & 1.88 & 0.093 & 10.7 & 50 & 64 & 0.60 & 1.13 & 4.31 & 44 & 0.39 \\
\hline 940222 & 0202 & 1.73 & 0.093 & 10.7 & 56 & 64 & 0.56 & 0.90 & 4.46 & 37 & 0.18 \\
\hline 940222 & 0510 & 1.62 & 0.093 & 10.7 & 50 & 63 & 0.56 & 1.06 & 4.73 & 37 & 0.42 \\
\hline 940222 & 0759 & 1.82 & 0.093 & 10.7 & 48 & 59 & 0.50 & 1.20 & 5.66 & 31 & 0.65 \\
\hline 940222 & 1112 & 2.41 & 0.093 & 10.7 & 44 & 55 & 0.45 & 1.33 & 5.91 & 28 & 0.82 \\
\hline 940222 & 1409 & 2.66 & 0.083 & 12.0 & 42 & 51 & 0.45 & 1.36 & 5.91 & 25 & 0.63 \\
\hline 940222 & 1794 & 2.85 & 0.074 & 13.6 & 44 & 50 & 0.44 & 1.49 & 7.32 & 20 & 0.41 \\
\hline 940222 & 2009 & 2.65 & 0.074 & 13.6 & 46 & 54 & 0.47 & 1.13 & 6.03 & 28 & 0.55 \\
\hline 940223 & 0209 & 2.19 & 0.074 & 13.6 & 44 & 53 & 0.45 & 1.29 & 7.07 & 26 & 0.48 \\
\hline 940223 & 0509 & 2.21 & 0.083 & 12.0 & 50 & 53 & 0.45 & 1.17 & 7.15 & 23 & 0.15 \\
\hline 940223 & 0809 & 2.07 & 0.083 & 12.0 & 46 & 54 & 0.45 & 1.48 & 7.51 & 23 & 0.65 \\
\hline 940223 & 1112 & 2.04 & 0.083 & 12.0 & 42 & 54 & 0.47 & 1.29 & 6.55 & 29 & 0.68 \\
\hline 940223 & 1409 & 1.96 & 0.083 & 12.0 & 40 & 53 & 0.49 & 1.24 & 6.44 & 31 & 0.28 \\
\hline 940223 & 1713 & 9.80 & 0.083 & 12.0 & 50 & 54 & 0.50 & 1.22 & 6.16 & 30 & 0.16 \\
\hline 940223 & 2009 & 1.61 & 0.083 & 12.0 & 48 & 57 & 0.53 & 1.63 & 6.35 & 27 & 0.63 \\
\hline 940224 & 0209 & 1.42 & 0.093 & 10.7 & 54 & 61 & 0.51 & 1.60 & 6.77 & 27 & 0.28 \\
\hline 940224 & 0508 & 1.34 & 0.074 & 13.6 & 50 & 62 & 0.55 & 1.70 & 5.75 & 30 & 0.39 \\
\hline 940224 & 1410 & 1.45 & 0.064 & 15.6 & 58 & 60 & 0.56 & 1.70 & 5.54 & 30 & 0.12 \\
\hline 940224 & 1707 & 1.34 & 0.064 & 15.6 & 54 & 64 & 0.69 & 1.59 & 4.52 & 36 & 0.30 \\
\hline 940224 & 2002 & 1.28 & 0.064 & 15.6 & 54 & 77 & 0.84 & 0.82 & 2.72 & 65 & 0.48 \\
\hline 940225 & 0247 & 1.30 & 0.074 & 13.6 & 54 & 79 & 0.68 & 9.43 & 3.65 & 43 & 0.76 \\
\hline 940225 & 0809 & 1.26 & 0.074 & 13.6 & 54 & 69 & 0.71 & 1.50 & 3.33 & 45 & 0.81 \\
\hline 940225 & 1128 & 1.04 & 0.074 & 13.6 & 50 & 80 & 0.83 & 1.21 & 2.51 & 78 & 1.32 \\
\hline 940226 & 0208 & 0.98 & 0.074 & 13.6 & 44 & 91 & 0.87 & 0.47 & 1.90 & 95 & 0.50 \\
\hline 940226 & 0510 & 0.98 & 0.074 & 13.6 & 148 & 94 & 0.84 & 0.38 & 1.97 & 88 & 0.52 \\
\hline 940226 & 1410 & 1.08 & 0.074 & 13.6 & 64 & 101 & 0.84 & 0.57 & 2.03 & 86 & 0.88 \\
\hline 940226 & 1708 & 1.26 & 0.074 & 13.6 & 60 & 88 & 0.78 & 1.07 & 2.52 & 71 & 1.02 \\
\hline 940226 & 2010 & 1.44 & 0.074 & 13.6 & 80 & 85 & 0.67 & 0.97 & 3.26 & 42 & 0.31 \\
\hline 940227 & 0850 & 1.51 & 0.074 & 13.6 & 64 & 80 & 0.67 & 0.96 & 3.54 & 44 & 0.38 \\
\hline 940227 & 1109 & 1.55 & 0.074 & 13.6 & 58 & 82 & 0.69 & & 3.10 & 53 & 0.37 \\
\hline 940227 & 1409 & 1.70 & 0.074 & 13.6 & 70 & 79 & 0.61 & 0.78 & 3.72 & 40 & 0.17 \\
\hline 940227 & 1709 & 1.79 & 0.074 & 13.6 & 60 & 79 & 0.60 & 0.94 & 4.18 & 37 & 0.29 \\
\hline 940227 & 2009 & 1.71 & 0.083 & 12.0 & 54 & 74 & 0.64 & 0.84 & 3.53 & 46 & 0.09 \\
\hline 940228 & 0207 & 1.92 & 0.083 & 12.0 & 60 & 70 & 0.57 & 0.52 & 3.91 & 43 & 0.02 \\
\hline 940228 & 0509 & 1.92 & 0.083 & 12.0 & 56 & 69 & 0.58 & 0.65 & & 45 & 0.32 \\
\hline 940228 & 0809 & 1.93 & 0.083 & 12.0 & 54 & 65 & 0.57 & 1.04 & 4.33 & 39 & 0.42 \\
\hline 940228 & 1413 & 1.95 & 0.083 & 12.0 & 54 & 67 & 0.58 & 0.89 & 3.85 & 41 & 0.45 \\
\hline 940228 & 1715 & 1.95 & 0.083 & 12.0 & 56 & 69 & 0.58 & 0.56 & 3.82 & 45 & 0.28 \\
\hline 940228 & 2009 & 1.91 & 0.083 & 12.0 & 68 & 66 & 0.58 & 0.44 & 3.86 & 43 & -0.08 \\
\hline 940301 & 0211 & 1.85 & 0.083 & 12.0 & 60 & 69 & 0.57 & 0.46 & 4.22 & 39 & 0.12 \\
\hline 940301 & 0511 & 1.83 & 0.093 & 10.7 & 60 & 68 & 0.54 & 0.56 & 4.63 & 38 & 0.17 \\
\hline 940301 & 0811 & 2.09 & 0.093 & 10.7 & 52 & 62 & 0.57 & 0.77 & 4.29 & 42 & 0.28 \\
\hline
\end{tabular}




\begin{tabular}{|c|c|c|c|c|c|c|c|c|c|c|c|}
\hline \multicolumn{12}{|c|}{ Table A1 (Continued) } \\
\hline Date & $\begin{array}{l}\text { Time } \\
\text { GMT }\end{array}$ & $\begin{array}{l}H_{m o} \\
m\end{array}$ & $\begin{array}{l}f_{p} \\
\mathrm{~Hz}\end{array}$ & $\begin{array}{l}T_{p} \\
\text { sec }\end{array}$ & $\begin{array}{l}\theta_{p} \\
\operatorname{deg}\end{array}$ & $\begin{array}{c}\theta_{0} \\
\text { deg }\end{array}$ & o & Y & $\delta$ & $\begin{array}{l}\Delta \theta \\
\operatorname{deg}\end{array}$ & $A$ \\
\hline 940301 & 1121 & 1.90 & 0.093 & 10.7 & 62 & 62 & 0.58 & 0.57 & 4.14 & 40 & -0.05 \\
\hline 940301 & 1409 & 2.01 & 0.064 & 15.6 & 64 & 67 & 0.58 & 0.62 & 4.16 & 37 & 0.36 \\
\hline 940301 & 1708 & 1.97 & 0.064 & 15.6 & 70 & 67 & 0.57 & 0.52 & 4.34 & 35 & -0.25 \\
\hline 940301 & 2006 & 2.07 & 0.064 & 15.6 & 68 & 66 & 0.55 & 0.78 & 4.79 & 36 & 0.09 \\
\hline 940302 & 0209 & 2.28 & 0.074 & 13.6 & 62 & 65 & 0.50 & 0.92 & 6.44 & 25 & 0.08 \\
\hline 940302 & 0509 & 2.26 & 0.074 & 13.6 & 60 & 65 & 0.53 & 0.75 & 5.42 & 33 & 0.17 \\
\hline 940302 & 1109 & 2.15 & 0.083 & 12.0 & 64 & 65 & 0.53 & 0.62 & 5.00 & 34 & -0.03 \\
\hline 940302 & 1406 & 2.10 & 0.083 & 12.0 & 64 & 67 & 0.50 & 0.79 & 6.11 & 29 & 0.09 \\
\hline 940302 & 1709 & 2.04 & 0.083 & 12.0 & 64 & 70 & 0.51 & 0.77 & 5.70 & 30 & 0.13 \\
\hline 940302 & 2009 & 2.07 & 0.083 & 12.0 & 58 & 67 & 0.56 & 0.53 & 4.53 & 41 & 0.03 \\
\hline 940303 & 0509 & 1.84 & 0.083 & 12.0 & 62 & 74 & 0.54 & 0.96 & 5.32 & 37 & 0.27 \\
\hline 940303 & 0804 & 2.00 & 0.083 & 12.0 & 56 & 70 & 0.53 & 0.76 & 4.82 & 39 & 0.45 \\
\hline 940303 & 1107 & 1.98 & 0.083 & 12.0 & 56 & 71 & 0.55 & 0.94 & 4.91 & 37 & 0.20 \\
\hline 940303 & 1408 & 2.00 & 0.083 & 12.0 & 84 & 74 & 0.51 & 0.82 & 5.56 & 33 & -0.04 \\
\hline 940303 & 1708 & 1.83 & 0.083 & 12.0 & 62 & 77 & 0.57 & 0.81 & 4.56 & 41 & 0.57 \\
\hline 940303 & 2006 & 1.79 & 0.083 & 12.0 & 54 & 72 & 0.58 & 0.78 & 4.36 & 42 & 0.21 \\
\hline 940304 & 0209 & 2.51 & 0.074 & 13.6 & 60 & 69 & 0.48 & 1.00 & 6.42 & 27 & 0.40 \\
\hline 940304 & 0508 & 2.53 & 0.074 & 13.6 & 56 & 69 & 0.50 & 1.12 & 6.01 & 31 & 0.58 \\
\hline 940304 & 0808 & 2.34 & 0.083 & 12.0 & 70 & 71 & 0.54 & 0.79 & 5.11 & 33 & 0.10 \\
\hline 940304 & 1120 & 2.20 & 0.083 & 12.0 & 62 & 68 & 0.55 & 0.93 & 5.28 & 34 & 0.24 \\
\hline 940304 & 1408 & 2.09 & 0.083 & 12.0 & 60 & 71 & 0.58 & 0.90 & 4.44 & 40 & 0.29 \\
\hline 940304 & 1715 & 1.98 & 0.083 & 12.0 & 54 & 75 & 0.60 & 0.94 & 4.26 & 44 & 0.32 \\
\hline 940304 & 2011 & 1.95 & 0.083 & 12.0 & 60 & 72 & 0.61 & 0.71 & 3.75 & 45 & 0.15 \\
\hline 940305 & 0810 & 1.72 & 0.093 & 10.7 & 58 & 78 & 0.70 & 1.07 & 3.29 & 53 & 0.45 \\
\hline 940305 & 1110 & 1.73 & 0.083 & 12.0 & 58 & 79 & 0.69 & 0.94 & 3.32 & 53 & 0.34 \\
\hline 940305 & 1410 & 1.86 & 0.083 & 12.0 & 50 & 78 & 0.66 & 0.82 & 3.17 & 53 & 0.17 \\
\hline 940305 & 1710 & 2.08 & 0.083 & 12.0 & 58 & 77 & 0.64 & 0.91 & 3.51 & 47 & 0.30 \\
\hline 940305 & 2008 & 1.93 & 0.083 & 12.0 & 60 & 73 & 0.63 & 1.14 & 3.87 & 42 & 0.51 \\
\hline 940306 & 0210 & 2.08 & 0.083 & 12.0 & 56 & 67 & 0.57 & 1.43 & 4.79 & 33 & 0.70 \\
\hline 940306 & 0510 & 2.02 & 0.083 & 12.0 & 48 & 67 & 0.6 & 1.2 & 4.0 & 44 & 0.91 \\
\hline 940306 & 0810 & 2.26 & 0.083 & 12.0 & 48 & 62 & 0.59 & 1.6 & 4.85 & 36 & 0.88 \\
\hline 940306 & 1110 & 2.58 & 0.083 & 12.0 & 46 & 63 & 0.60 & 1.72 & 4.81 & 38 & 0.71 \\
\hline 940306 & 1410 & 2.99 & 0.083 & 12.0 & 52 & 58 & & 1.10 & 5.68 & 29 & 0.21 \\
\hline 940306 & 1710 & 2.90 & 0.083 & 12.0 & 46 & 56 & 0.58 & 1.68 & 5.23 & 34 & 0.67 \\
\hline 940306 & 2008 & 2.27 & 0.083 & 12.0 & 44 & 62 & 0.62 & 1.76 & 4.89 & 37 & 0.61 \\
\hline 940307 & 0210 & 2.66 & 0.083 & 12.0 & 44 & 55 & 0.55 & 1.56 & 5.56 & 33 & 0.43 \\
\hline & 0510 & 2.68 & 0.083 & 12.0 & 44 & 52 & 0.51 & 1.89 & 6.78 & 25 & 0.62 \\
\hline 940307 & 1125 & 2.24 & 0.083 & 12.0 & 44 & 55 & 0.52 & 1.61 & 6.05 & 30 & 0.61 \\
\hline 940308 & 0208 & 1.74 & 0.093 & 10.7 & 44 & 56 & 0.54 & 1.61 & 5.89 & 33 & 0.81 \\
\hline 940308 & 0511 & & 0.093 & 10.7 & 40 & 55 & & 1.6 & 5.7 & 33 & 0.74 \\
\hline 940308 & 0811 & 1.37 & 0.093 & 10.7 & 40 & 56 & 0.61 & 1.82 & 4.99 & 36 & 0.94 \\
\hline 940308 & 1109 & & 0.093 & 10.7 & 38 & 57 & & 1.53 & 5.22 & 38 & 0.68 \\
\hline 940308 & 1410 & 1.49 & 0.103 & 9.7 & 40 & 52 & 0.56 & 1.68 & 5.39 & 32 & 0.64 \\
\hline 940308 & 1716 & 1.23 & 0.103 & 9.7 & 38 & 57 & 0.61 & 1.67 & 4.89 & 40 & 0.92 \\
\hline 940308 & 2010 & 1.02 & 0.103 & 9.7 & 34 & 56 & 0.67 & 1.88 & 4.25 & 43 & 0.88 \\
\hline 940309 & 0210 & 0.94 & 0.113 & 8.9 & 44 & 62 & 0.63 & 1.87 & 4.63 & 36 & 0.33 \\
\hline & 0510 & 0.89 & 0.123 & 8.2 & 44 & 65 & 0.68 & 1.72 & 4.11 & 40 & 0.32 \\
\hline 940309 & 0810 & 0.87 & 0.113 & 8.9 & 42 & 62 & 0.68 & 2.13 & 4.46 & 37 & 0.49 \\
\hline 940309 & 1104 & 0.80 & 0.064 & 15.6 & 38 & 68 & 0.76 & 1.66 & 3.42 & 49 & 0.29 \\
\hline 940309 & 1410 & 0.94 & 0.064 & 15.6 & 76 & 74 & 0.71 & 1.65 & 3.78 & 38 & -0.15 \\
\hline 940309 & 1705 & 0.94 & 0.064 & 15.6 & 72 & 74 & 0.71 & 1.56 & 3.68 & 37 & 0.15 \\
\hline 940309 & 2008 & 1.15 & 0.074 & 13.6 & 54 & 73 & 0.67 & 1.53 & 4.18 & 41 & 0.40 \\
\hline & & & & & & & & & & & \\
\hline
\end{tabular}




\begin{tabular}{|c|c|c|c|c|c|c|c|c|c|c|c|}
\hline \multicolumn{12}{|c|}{ Table A1 (Continued) } \\
\hline Date & $\begin{array}{l}\text { Time } \\
\text { GMT }\end{array}$ & $\begin{array}{l}H_{m o} \\
m\end{array}$ & $\begin{array}{l}f_{p} \\
\mathrm{~Hz}\end{array}$ & $\begin{array}{l}T_{p} \\
\text { sec }\end{array}$ & $\begin{array}{l}\theta_{p} \\
\text { deg }\end{array}$ & $\begin{array}{l}\theta_{0} \\
\text { deg }\end{array}$ & $\sigma$ & Y & $\delta$ & $\begin{array}{l}\Delta \theta \\
\operatorname{deg}\end{array}$ & $A$ \\
\hline 940310 & 0210 & 1.11 & 0.074 & 13.6 & 60 & 75 & 0.68 & 1.84 & 4.24 & 35 & 0.52 \\
\hline 940310 & 0510 & 1.01 & 0.074 & 13.6 & 54 & 79 & 0.74 & 1.47 & 3.40 & 46 & 0.21 \\
\hline 940310 & 1109 & 1.03 & 0.074 & 13.6 & 56 & 86 & 0.80 & 1.34 & 2.82 & 57 & 0.52 \\
\hline 940310 & 1409 & 1.25 & 0.083 & 12.0 & 82 & 80 & 0.71 & 1.36 & 3.52 & 43 & -0.14 \\
\hline 940310 & 1707 & 1.20 & 0.083 & 12.0 & 56 & 76 & 0.71 & 1.81 & 3.80 & 41 & 0.50 \\
\hline 940310 & 2010 & 1.15 & 0.083 & 12.0 & 48 & 75 & 0.79 & 1.70 & 3.13 & 54 & 0.96 \\
\hline 940311 & 0508 & 2.02 & 0.142 & 7.0 & 50 & 57 & 0.61 & 1.48 & 4.82 & 41 & 0.45 \\
\hline 940311 & 0810 & 2.69 & 0.132 & 7.6 & 48 & 52 & 0.55 & 1.51 & 5.71 & 34 & 0.22 \\
\hline 940311 & 1059 & 2.52 & 0.074 & 13.6 & 52 & 59 & 0.51 & 1.86 & 6.79 & 25 & 0.38 \\
\hline 940311 & 1350 & 2.73 & 0.123 & 8.2 & 48 & 59 & 0.51 & 1.37 & 5.87 & 32 & 0.40 \\
\hline 940311 & 1700 & 2.81 & 0.123 & 8.2 & 54 & 58 & 0.54 & 1.28 & 5.62 & 35 & 0.12 \\
\hline 940312 & 0203 & 2.99 & 0.083 & 12.0 & 50 & 62 & 0.54 & 1.56 & 5.81 & 32 & 0.51 \\
\hline 940312 & 0509 & 2.73 & 0.074 & 13.6 & 54 & 62 & 0.57 & 1.57 & 5.58 & 34 & 0.42 \\
\hline 940312 & 0809 & 2.53 & 0.074 & 13.6 & 52 & 65 & 0.66 & 1.90 & 4.64 & 37 & 0.78 \\
\hline 940313 & 0209 & 1.98 & 0.083 & 12.0 & 56 & 77 & 0.71 & 1.40 & 3.46 & 47 & 0.52 \\
\hline 940313 & 0509 & 1.73 & 0.074 & 13.6 & 52 & 86 & 0.82 & 0.90 & 2.56 & 67 & 0.34 \\
\hline 940313 & 1108 & 1.55 & 0.064 & 15.6 & 56 & 88 & 0.82 & 0.85 & 2.41 & 71 & 0.53 \\
\hline 940313 & 1408 & 1.54 & 0.064 & 15.6 & 54 & 89 & 0.77 & 0.76 & 2.74 & 61 & 0.18 \\
\hline 940313 & 1704 & 1.55 & 0.064 & 15.6 & 60 & 98 & 0.88 & 0.49 & 2.08 & 88 & 0.48 \\
\hline 940313 & 2008 & 1.65 & 0.064 & 15.6 & 78 & 97 & 0.85 & 0.83 & 2.19 & 83 & 1.04 \\
\hline 940314 & 0209 & 1.86 & 0.074 & 13.6 & 62 & 87 & 0.75 & 1.10 & 2.82 & 60 & 0.94 \\
\hline 940314 & 0509 & 2.03 & 0.074 & 13.6 & 62 & 87 & 0.78 & 1.22 & 2.81 & 58 & 0.72 \\
\hline 940314 & 0808 & 1.98 & 0.074 & 13.6 & 56 & 84 & 0.83 & 1.24 & 2.53 & 73 & 1.17 \\
\hline 940314 & 1108 & 2.05 & 0.074 & 13.6 & 56 & 82 & 0.79 & 1.43 & 2.82 & 58 & 0.94 \\
\hline 940314 & 1408 & 2.11 & 0.074 & 13.6 & 56 & 93 & 0.84 & 0.97 & 2.34 & 77 & 0.55 \\
\hline 940314 & 1708 & 1.96 & 0.083 & 12.0 & 58 & 84 & 0.81 & 1.46 & 2.79 & 57 & 0.64 \\
\hline 940314 & 2008 & 1.95 & 0.083 & 12.0 & 70 & 92 & 0.82 & 0.91 & 2.45 & 67 & 0.66 \\
\hline 940315 & 0209 & 1.85 & 0.083 & 12.0 & 58 & 89 & 0.75 & 1.00 & 2.80 & 58 & 0.20 \\
\hline 940315 & 0500 & 1.80 & 0.083 & 12.0 & 56 & 86 & 0.77 & 1.07 & 2.78 & 56 & 0.10 \\
\hline 940315 & 0808 & 1.55 & 0.083 & 12.0 & 52 & 92 & 0.87 & 0.94 & 2.22 & 90 & 0.61 \\
\hline 940315 & 1108 & 1.71 & 0.083 & 12.0 & 66 & 94 & 0.79 & 0.69 & 2.49 & 67 & 0.40 \\
\hline 940315 & 1408 & 1.64 & 0.093 & 10.7 & 68 & 92 & 0.78 & 0.91 & 2.64 & 59 & 0.35 \\
\hline 940315 & 1713 & 1.54 & 0.074 & 13.6 & 96 & 92 & 0.80 & 0.65 & 2.44 & 69 & -0.06 \\
\hline 940315 & 2005 & 1.52 & 0.074 & 13.6 & 94 & 96 & 0.80 & 0.68 & 2.39 & 72 & 0.30 \\
\hline 940316 & 0205 & 1.68 & 0.083 & 12.0 & 104 & 97 & 0.72 & 0.83 & 2.92 & 51 & -0.11 \\
\hline 940316 & 0509 & 1.69 & 0.083 & 12.0 & 62 & 92 & 0.72 & 0.77 & 2.89 & 54 & 0.02 \\
\hline 940316 & 0808 & 2.01 & 0.054 & 18.5 & 62 & 86 & 0.69 & 1.06 & 3.29 & 47 & 0.44 \\
\hline 940316 & 1108 & 2.14 & 0.054 & 18.5 & 62 & 83 & 0.71 & 1.16 & 3.10 & 53 & 0.87 \\
\hline 940316 & 1409 & 2.40 & 0.064 & 15.6 & 58 & 76 & 0.65 & 1.52 & 3.98 & 44 & 1.43 \\
\hline 940316 & 1708 & 2.65 & 0.064 & 15.6 & 64 & 74 & 0.58 & 1.57 & 5.04 & 29 & 0.86 \\
\hline 940316 & 2008 & 2.42 & 0.064 & 15.6 & 60 & 78 & 0.66 & 1.29 & 3.94 & 42 & 0.73 \\
\hline 940317 & 0209 & 2.37 & 0.074 & 13.6 & 58 & 73 & 0.65 & 1.33 & 3.84 & 45 & 1.11 \\
\hline 940317 & 0508 & 2.78 & 0.064 & 15.6 & 60 & 67 & 0.56 & 1.50 & 5.08 & 28 & 0.46 \\
\hline 940317 & 0805 & 2.69 & 0.074 & 13.6 & 50 & 63 & 0.58 & 1.78 & 5.22 & 31 & 0.52 \\
\hline 940317 & 1108 & 2.72 & 0.074 & 13.6 & 54 & 63 & 0.57 & 1.83 & 5.67 & 29 & 0.33 \\
\hline 940317 & 1421 & 2.87 & 0.074 & 13.6 & 50 & 62 & 0.53 & 2.03 & 6.75 & 27 & 0.58 \\
\hline 940317 & 2152 & 2.60 & 0.074 & 13.6 & 54 & 63 & 0.60 & 1.99 & 5.56 & 31 & 0.27 \\
\hline 940318 & 0208 & 2.23 & 0.074 & 13.6 & 52 & 60 & 0.58 & 2.91 & 6.57 & 22 & 0.64 \\
\hline 940318 & 0509 & 2.17 & 0.074 & 13.6 & 50 & 68 & 0.67 & 2.09 & 4.56 & 39 & 0.64 \\
\hline 940318 & 0808 & 2.08 & 0.074 & 13.6 & 50 & 66 & 0.70 & 1.79 & 4.12 & 42 & 0.72 \\
\hline 940318 & 1100 & 1.91 & 0.083 & 12.0 & 48 & 62 & 0.71 & 2.45 & 4.45 & 37 & 0.85 \\
\hline 940318 & 2009 & 1.97 & 0.083 & 12.0 & 54 & 67 & 0.66 & 2.25 & 4.65 & 33 & 0.59 \\
\hline
\end{tabular}




\begin{tabular}{|c|c|c|c|c|c|c|c|c|c|c|c|}
\hline Date & $\begin{array}{l}\text { Time } \\
\text { GMT }\end{array}$ & $\begin{array}{l}H_{m 0} \\
\mathrm{~m}\end{array}$ & $\begin{array}{l}f_{p} \\
\mathrm{~Hz}\end{array}$ & $\begin{array}{l}T_{p} \\
\text { sec }\end{array}$ & $\begin{array}{l}\theta_{p} \\
\text { deg }\end{array}$ & $\begin{array}{c}\theta_{0} \\
\operatorname{deg}\end{array}$ & $\sigma$ & Y & $\delta$ & $\begin{array}{l}\Delta \theta \\
\operatorname{deg}\end{array}$ & A \\
\hline 940319 & 0209 & 2.09 & 0.083 & 12.0 & 52 & 62 & 0.58 & 2.54 & 6.15 & 26 & 0.55 \\
\hline 940319 & 0809 & .95 & .083 & 12.0 & 58 & 66 & 0.62 & 2.85 & & 24 & 0.62 \\
\hline 940319 & 1108 & 1.80 & 0.083 & 12.0 & 50 & 67 & 0.74 & 2.53 & 4.26 & 38 & 0.94 \\
\hline 940319 & 1408 & 2.15 & 0.083 & 12.0 & 50 & 61 & 0.59 & 2.93 & 6.26 & 23 & 0.77 \\
\hline 940319 & 1708 & 2.47 & 0.083 & 12.0 & 50 & 61 & 0.52 & 2.12 & 6.70 & 25 & 0.63 \\
\hline 940319 & 2008 & 2.77 & 0.074 & 13.6 & 48 & 57 & 0.51 & 2.32 & 7.47 & 23 & 0.43 \\
\hline 940319 & 2304 & 2.90 & 0.074 & 13.6 & 50 & 57 & 0.48 & 2.08 & 7.83 & 23 & 0.45 \\
\hline 940320 & 0208 & 3.19 & 0.074 & 13.6 & 52 & 58 & 0.43 & 2.39 & 9.64 & 18 & 0.43 \\
\hline 940320 & 0509 & 3.52 & 0.074 & 13.6 & 48 & 58 & 0.47 & 2.07 & 7.95 & 23 & 0.71 \\
\hline 940320 & 0808 & 3.56 & 0.074 & 13.6 & 50 & 58 & 0.48 & 2.12 & 7.88 & 23 & 0.69 \\
\hline 940320 & 1108 & 3.22 & 0.074 & 13.6 & 48 & 56 & 0.46 & 2.40 & 9.07 & 21 & 0.48 \\
\hline 940320 & 1408 & 3.20 & 0.074 & 13.6 & 52 & 58 & 0.42 & 2.33 & 10.47 & 16 & 0.39 \\
\hline 940320 & 1708 & 2.90 & 0.074 & 13.6 & 48 & 56 & 0.48 & 2.62 & 8.89 & 19 & 0.67 \\
\hline 940320 & 2008 & 2.55 & 0.074 & 13.6 & 50 & 59 & 0.53 & 2.44 & 7.29 & 24 & 0.59 \\
\hline 940321 & 0208 & 2.56 & 0.074 & 13.6 & 50 & 58 & 0.45 & 2.99 & 10.07 & 16 & 0.52 \\
\hline 940321 & 0509 & 2.40 & 0.074 & 13.6 & 52 & 61 & 0.53 & 2.61 & 7.29 & 22 & 0.51 \\
\hline 940321 & 0808 & 2.47 & 0.074 & 13.6 & 52 & 60 & 0.48 & 2.08 & 8.02 & 24 & 0.41 \\
\hline 940321 & 1108 & 2.13 & 0.083 & 12.0 & 50 & 59 & 0.52 & 2.88 & 7.82 & 23 & 0.47 \\
\hline 940321 & 1408 & 1.99 & 0.083 & 12.0 & 50 & 58 & 0.55 & 3.30 & 7.40 & 20 & 0.69 \\
\hline 940321 & 1709 & 1.75 & 0.083 & 12.0 & 52 & 65 & 0.65 & 2.51 & 5.20 & 31 & 0.64 \\
\hline 940321 & 2008 & 1.97 & 0.083 & 12.0 & 52 & 62 & 0.61 & 1.96 & 5.58 & 30 & 0.28 \\
\hline 940322 & 0158 & 2.94 & 0.083 & 12.0 & 46 & 53 & 0.52 & 2.27 & 7.19 & 24 & 0.49 \\
\hline 940322 & 0509 & 3.43 & 0.074 & 13.6 & 48 & 54 & 0.45 & 1.69 & 8.45 & 22 & 0.34 \\
\hline 940322 & 0807 & 3.98 & 0.074 & 13.6 & 52 & 53 & 0.48 & 1.08 & 7.11 & 26 & 0.03 \\
\hline 940322 & 1408 & 3.40 & 0.074 & 13.6 & 50 & 55 & 0.45 & 1.56 & 8.40 & 21 & 0.31 \\
\hline 940322 & 1709 & 3.58 & 0.074 & 13.6 & 52 & 58 & 0.47 & 1.78 & 8.04 & 21 & 0.35 \\
\hline 940323 & 0213 & 3.83 & 0.064 & 15.6 & 56 & 57 & 0.41 & 1.16 & 9.44 & 19 & 0.07 \\
\hline 940323 & 0513 & 3.58 & 0.064 & 15.6 & 56 & 57 & 0.41 & 1.80 & 10.57 & 14 & -0.03 \\
\hline 940323 & 0811 & 3.55 & 0.064 & 15.6 & 56 & 59 & 0.44 & 1.84 & 9.12 & 17 & 0.33 \\
\hline 940323 & 1112 & 3.41 & 0.074 & 13.6 & 52 & 58 & 0.44 & 1.50 & 8.38 & 21 & 0.36 \\
\hline 940323 & 1413 & 3.53 & 0.074 & 13.6 & 52 & 57 & 0.48 & 1.72 & 7.85 & 23 & 0.30 \\
\hline 940323 & 1702 & 3.23 & 0.074 & 13.6 & 50 & 55 & 0.47 & 1.85 & 8.13 & 21 & 0.36 \\
\hline 940323 & 2013 & 2.78 & 0.074 & 13.6 & 54 & 58 & 0.51 & 1.55 & 6.60 & 27 & 0.19 \\
\hline 940324 & 0213 & 2.22 & 0.074 & 13.6 & 52 & 64 & 0.57 & 1.72 & 5.54 & 32 & 0.35 \\
\hline 940324 & 0511 & 1.78 & 0.074 & 13.6 & 48 & 70 & & 1.55 & & 43 & 0.43 \\
\hline 940324 & 0813 & 1.50 & 0.083 & 12.0 & 50 & $\pi 7$ & 0.82 & 1.51 & 3.06 & 57 & 0.68 \\
\hline 940324 & 1201 & 2.09 & .162 & & -106 & -176 & 1.18 & -1.25 & 1.85 & 151 & -0.87 \\
\hline 940324 & 1409 & 2.78 & 0.152 & 6.6 & -180 & 171 & 1.12 & -0.59 & 1.73 & 138 & -0.49 \\
\hline 940324 & 1714 & 3.14 & 0.132 & 7.6 & 74 & 131 & 0.97 & 0.28 & 1.96 & 97 & -0.11 \\
\hline 940324 & 2013 & 2.94 & 0.123 & 8.2 & 60 & 110 & 0.86 & 0.36 & 1.98 & 92 & 0.05 \\
\hline 940325 & 0213 & 2.85 & 0.083 & 12.0 & 54 & 82 & 0.74 & 1.07 & 2.75 & 66 & 1.15 \\
\hline 940325 & 0513 & 3.36 & 0.074 & 13.6 & 56 & 77 & 0.71 & 1.29 & 3.17 & 54 & 1.11 \\
\hline 940325 & 0813 & & 0.074 & & 56 & 80 & & 1.24 & & 58 & 0.95 \\
\hline & 1113 & 2.72 & 0.083 & 12.0 & 58 & 81 & 0.73 & & & 58 & 0.96 \\
\hline 940325 & 1411 & 2.52 & 0.083 & 12.0 & 60 & 84 & 0.72 & 0.82 & 2.91 & 58 & 0.48 \\
\hline 940325 & 1712 & 2.24 & 0.083 & 12.0 & 60 & 91 & 0.72 & 0.62 & 2.65 & 63 & 0.62 \\
\hline 940325 & 2003 & 2.17 & 0.064 & 15.6 & 60 & 87 & 0.73 & 0.88 & 2.75 & 63 & 1.06 \\
\hline 940326 & 0202 & 2.11 & 0.06 & 15. & 58 & 81 & 0.72 & 1.21 & 2. & 61 & 1.37 \\
\hline & 0503 & & 0.074 & 13.6 & 56 & 79 & 0.71 & 1.01 & 2. & 61 & 9.05 \\
\hline 940326 & 0803 & 2.17 & 0.074 & 13.6 & 54 & 75 & 0.68 & 1.18 & 3.32 & 52 & 0.90 \\
\hline 940326 & 1103 & 2.15 & 0.074 & 13.6 & 56 & 74 & 0.65 & 1.28 & 3.82 & 42 & 0.63 \\
\hline 940326 & 1403 & 2.21 & 0.074 & 13.6 & 54 & 70 & 0.66 & 1.46 & 4.05 & 37 & 0.25 \\
\hline 940326 & 1702 & 2.04 & 0.044 & 22.5 & 76 & 78 & 0.74 & 1.35 & 3.25 & 43 & 0.22 \\
\hline
\end{tabular}

(Sheet 10 of 45) 


\begin{tabular}{|c|c|c|c|c|c|c|c|c|c|c|c|}
\hline \multicolumn{12}{|c|}{ Table A1 (Continued) } \\
\hline Date & $\begin{array}{l}\text { Time } \\
\text { GMT }\end{array}$ & $\begin{array}{l}H_{m o} \\
\mathrm{~m}\end{array}$ & $\begin{array}{l}f_{p} \\
\mathrm{~Hz}\end{array}$ & $\begin{array}{l}T_{p} \\
\text { sec }\end{array}$ & $\begin{array}{l}\theta_{p} \\
\operatorname{deg}\end{array}$ & $\begin{array}{l}\theta_{0} \\
\text { deg }\end{array}$ & $\sigma$ & v & $\delta$ & $\begin{array}{l}\Delta \theta \\
\operatorname{deg}\end{array}$ & A \\
\hline $\begin{array}{l}940327 \\
940327\end{array}$ & \begin{tabular}{|l|}
0203 \\
1924
\end{tabular} & $\begin{array}{l}2.19 \\
1.91\end{array}$ & $\begin{array}{l}0.054 \\
0.064\end{array}$ & \begin{tabular}{|l|}
18.5 \\
15.6
\end{tabular} & $\begin{array}{l}68 \\
68\end{array}$ & $\begin{array}{l}72 \\
83\end{array}$ & $\begin{array}{l}0.63 \\
0.87\end{array}$ & $\begin{array}{l}1.98 \\
1.39\end{array}$ & $\begin{array}{l}4.79 \\
2.50\end{array}$ & $\begin{array}{l}21 \\
81\end{array}$ & $\begin{array}{l}0.27 \\
1.48\end{array}$ \\
\hline 940328 & 0200 & 2.40 & 0.064 & 15.6 & 66 & 76 & 0.67 & 2.09 & 4.29 & 26 & 0.74 \\
\hline 940328 & 0759 & 2.14 & 0.064 & 15.6 & 64 & $\pi$ & 0.79 & 2.16 & 3.94 & 30 & 0.87 \\
\hline 940328 & 1103 & 1.82 & 0.064 & 15.6 & 64 & 84 & 0.84 & 1.75 & 2.90 & 63 & 1.70 \\
\hline 940328 & 1411 & 1.89 & 0.064 & 15.6 & 68 & 81 & 0.75 & 1.61 & 3.28 & 40 & 1.08 \\
\hline 940328 & 1713 & 1.73 & 0.064 & 15.6 & 66 & 79 & 0.77 & 1.85 & 3.23 & 46 & 0.93 \\
\hline 940328 & 2009 & 1.72 & 0.074 & 13.6 & 70 & 87 & 0.80 & 1.17 & 2.61 & 71 & 1.41 \\
\hline 940329 & 0213 & 1.62 & 0.074 & 13.6 & 60 & 85 & 0.78 & 1.12 & 2.60 & 68 & 1.06 \\
\hline 940329 & 0513 & 1.33 & 0.074 & 13.6 & 46 & 90 & 0.86 & 0.82 & 2.28 & 85 & 0.50 \\
\hline 940329 & 0813 & 1.31 & 0.074 & 13.6 & 64 & 92 & 0.83 & 1.05 & 2.35 & 80 & 1.11 \\
\hline 940329 & 1113 & 1.30 & 0.074 & 13.6 & 82 & 100 & 0.77 & 0.29 & 2.53 & 65 & 0.58 \\
\hline 940329 & 1411 & 1.25 & 0.074 & 13.6 & 60 & 90 & 0.74 & 0.89 & 2.80 & 59 & 0.49 \\
\hline 940329 & 1711 & 1.28 & 0.074 & 13.6 & 88 & 96 & 0.75 & 0.52 & 2.45 & 66 & 0.30 \\
\hline 940329 & 2013 & 1.28 & 0.074 & 13.6 & 60 & 93 & 0.74 & 0.80 & 2.60 & 63 & 0.43 \\
\hline 940330 & 0213 & 1.58 & 0.083 & 12.0 & 72 & 88 & 0.63 & 0.92 & 3.71 & 42 & 0.39 \\
\hline 940330 & 0511 & 1.69 & 0.083 & 12.0 & 68 & 83 & 0.60 & 0.94 & 3.99 & 40 & 0.46 \\
\hline 940330 & 0813 & 2.01 & 0.083 & 12.0 & 82 & 75 & 0.59 & 0.60 & 4.10 & 37 & -0.31 \\
\hline 940330 & 1116 & 2.15 & 0.093 & 10.7 & 56 & 73 & 0.59 & 0.74 & 3.79 & 43 & 0.48 \\
\hline 940330 & 1415 & 2.28 & 0.103 & 9.7 & 54 & 79 & 0.61 & 0.71 & 3.42 & 49 & 0.63 \\
\hline 940330 & 1714 & 2.49 & 0.142 & 7.0 & 52 & 67 & 0.59 & 0.68 & 3.78 & 46 & 0.26 \\
\hline 940330 & 2013 & 2.93 & 0.132 & 7.6 & 58 & 59 & 0.53 & 0.69 & 4.37 & 40 & 0.07 \\
\hline 940331 & 0211 & 2.73 & 0.132 & 7.6 & 56 & 58 & 0.55 & 1.01 & 4.56 & 39 & 0.10 \\
\hline 940331 & 0513 & 2.68 & 0.123 & 8.2 & 50 & 63 & 0.56 & 9.10 & 4.55 & 40 & 0.39 \\
\hline 940331 & 0813 & 2.47 & 0.113 & 8.9 & 56 & 62 & 0.55 & 1.37 & 5.38 & 33 & 0.35 \\
\hline 940331 & 1113 & 2.35 & 0.064 & 15.6 & 56 & 63 & 0.57 & 1.52 & 5.16 & 31 & 0.40 \\
\hline 940331 & 1413 & 2.42 & 0.074 & 13.6 & 52 & 59 & 0.58 & 1.46 & 5.21 & 29 & 0.55 \\
\hline 940331 & 1713 & 2.50 & 0.074 & 13.6 & 56 & 65 & 0.58 & 1.1 & 4.73 & 33 & 0.54 \\
\hline 940331 & 2013 & 2.84 & 0.074 & 13.6 & 54 & 65 & 0.55 & 1.21 & 5.09 & 35 & 0.29 \\
\hline 940401 & 0211 & 2.65 & 0.074 & 13.6 & 56 & 66 & 0.54 & 1.60 & 5.82 & 28 & 0.50 \\
\hline 940401 & 0511 & 2.64 & 0.074 & 13.6 & 54 & 62 & 0.5 & 1.4 & 5.4 & 32 & 0.49 \\
\hline 940401 & 0813 & 2.36 & 0.074 & 13.6 & 54 & 66 & 0.55 & 1.2 & 5.07 & 36 & 0.58 \\
\hline 940401 & 1114 & 2.38 & 0.083 & 12.0 & 56 & 68 & 0.57 & 1.47 & 5.13 & 35 & 0.64 \\
\hline 940401 & 1415 & 2.48 & 0.054 & 18.5 & 78 & 72 & 0.56 & 1.2 & 5.12 & 32 & -0.53 \\
\hline 940401 & 2011 & 2.99 & 0.054 & 18.5 & 78 & 74 & 0.49 & 0.99 & 6.29 & 22 & -0.55 \\
\hline 940402 & 0513 & 3.24 & 0.064 & 15.6 & 74 & 74 & 0.48 & 0.91 & 6.44 & 24 & -0.02 \\
\hline 940402 & 0811 & 3.55 & 0.064 & 15.6 & 76 & 76 & 0.4 & 1.0 & 7.1 & 20 & -0.07 \\
\hline 940402 & 1113 & 3.45 & 0.064 & 15.6 & 76 & 75 & 0.47 & 1.21 & 7.20 & 20 & -0.21 \\
\hline 940402 & 1413 & 3.21 & 0.064 & 15.6 & 76 & 75 & 0.47 & 0.95 & 6.38 & 22 & -0.19 \\
\hline 940402 & 1711 & 3.31 & 0.064 & 15.6 & 70 & 75 & 0.48 & 0.88 & 5.98 & 24 & 0.36 \\
\hline 940402 & 2013 & 3.15 & 0.064 & 15.6 & 74 & 72 & 0.47 & 0.98 & 6.48 & 25 & -0.19 \\
\hline 940403 & 0219 & 3.00 & 0.064 & 15.6 & 56 & 70 & 0.53 & 0.92 & 4.81 & 34 & 0.16 \\
\hline 940403 & 0511 & 3.02 & 0.074 & 13.6 & 58 & 66 & 0.51 & 0.9 & 5.49 & 31 & 0.18 \\
\hline 940403 & 0813 & 3.08 & 0.074 & 13.6 & 58 & 69 & 0.5 & 0.7 & 4.7 & 34 & 0.22 \\
\hline 940403 & 1910 & 3.15 & 0.074 & 13.6 & 60 & 64 & 0.49 & 0.8 & 5.32 & 28 & 0.27 \\
\hline 940403 & 1410 & 2.91 & 0.074 & 13.6 & 58 & 64 & 0.54 & 1.0 & 4.64 & 32 & 0.23 \\
\hline 940403 & 1713 & 2.81 & 0.074 & 13.6 & 56 & 64 & 0.55 & 1.24 & 4.61 & 33 & 0.43 \\
\hline 940403 & 2010 & 2.66 & 0.074 & 13.6 & 56 & 61 & 0.56 & 1.01 & 4.63 & 38 & 0.24 \\
\hline 940404 & 0211 & & & & 56 & 61 & 0.57 & 1.0 & 4.14 & 38 & 0.09 \\
\hline 940404 & 0513 & 2.82 & 0.083 & 12.0 & 54 & 61 & 0.49 & 1.2 & 5.61 & 28 & 0.28 \\
\hline 940404 & 0816 & 2.60 & 0.083 & 12.0 & 54 & 61 & 0.49 & 0.96 & 5.42 & 33 & 0.22 \\
\hline 940404 & 1113 & 2.58 & 0.083 & 12.0 & 58 & 59 & 0.50 & 1.14 & 5.68 & 31 & 0.00 \\
\hline
\end{tabular}




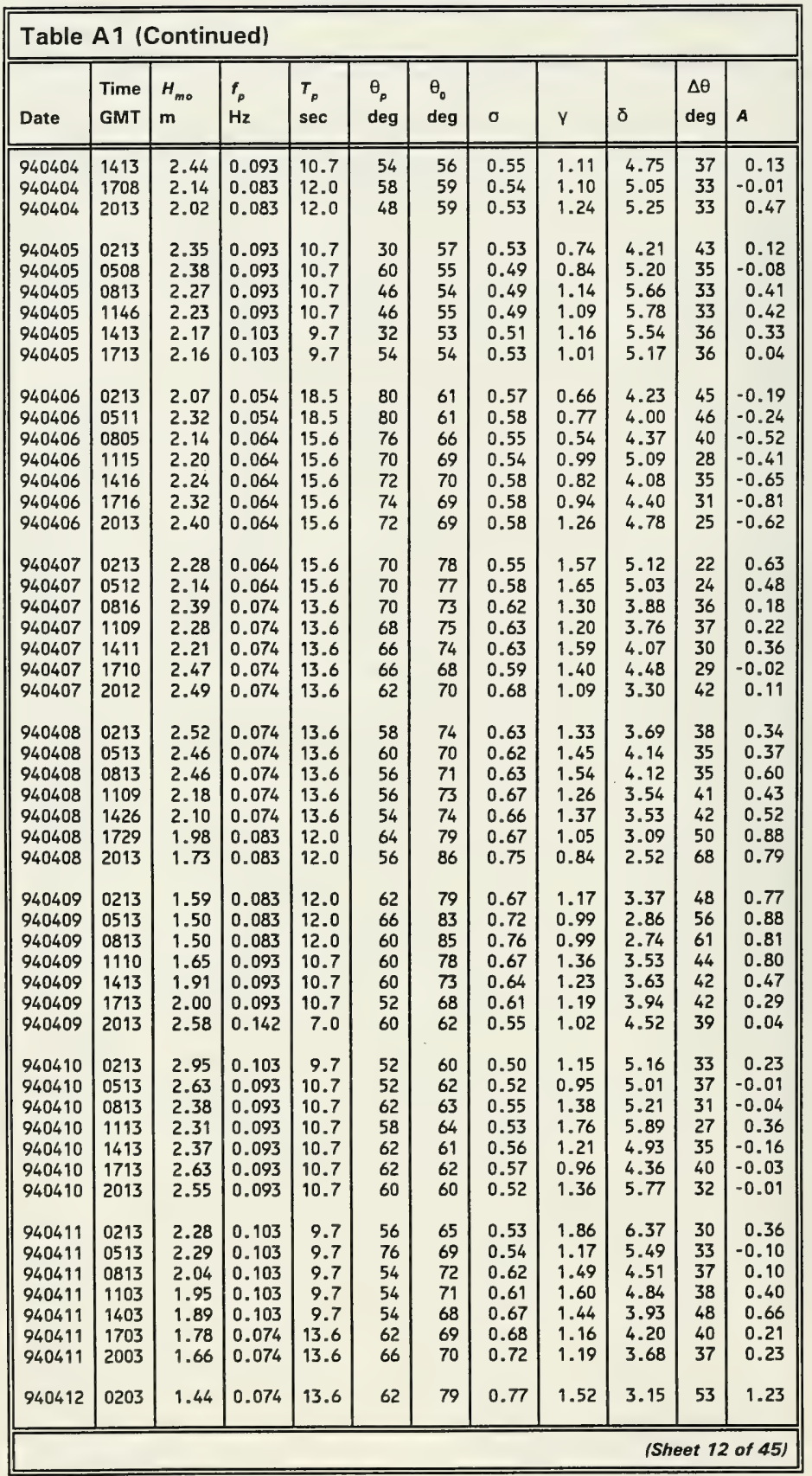




\begin{tabular}{|c|c|c|c|c|c|c|c|c|c|c|c|}
\hline Date & $\begin{array}{l}\text { Time } \\
\text { GMT }\end{array}$ & $\begin{array}{l}H_{m 0} \\
\mathrm{~m}\end{array}$ & $\begin{array}{l}f_{p} \\
H z\end{array}$ & $\begin{array}{l}T_{p} \\
\text { sec }\end{array}$ & $\begin{array}{l}\theta_{p} \\
\text { deg }\end{array}$ & $\begin{array}{c}\theta_{0} \\
\text { deg }\end{array}$ & $\sigma$ & $Y$ & $\delta$ & $\begin{array}{c}\Delta \theta \\
\operatorname{deg}\end{array}$ & A \\
\hline $\begin{array}{l}940412 \\
940412 \\
940412 \\
940412 \\
940412 \\
940412\end{array}$ & $\begin{array}{l}0503 \\
0803 \\
1103 \\
1359 \\
1702 \\
2003\end{array}$ & $\begin{array}{l}1.42 \\
1.38 \\
1.47 \\
1.35 \\
1.38 \\
1.42\end{array}$ & $\begin{array}{l}0.074 \\
0.064 \\
0.074 \\
0.074 \\
0.074 \\
0.074\end{array}$ & $\begin{array}{l}13.6 \\
15.6 \\
13.6 \\
13.6 \\
13.6 \\
13.6\end{array}$ & $\begin{array}{l}64 \\
62 \\
66 \\
58 \\
66 \\
64\end{array}$ & $\begin{array}{l}78 \\
75 \\
79 \\
80 \\
77 \\
75\end{array}$ & $\begin{array}{l}0.75 \\
0.74 \\
0.75 \\
0.80 \\
0.65 \\
0.67\end{array}$ & $\begin{array}{l}1.74 \\
1.63 \\
1.25 \\
1.50 \\
1.39 \\
1.87\end{array}$ & $\begin{array}{l}3.49 \\
3.50 \\
2.94 \\
2.99 \\
4.06 \\
4.26\end{array}$ & $\begin{array}{l}38 \\
40 \\
52 \\
53 \\
35 \\
31\end{array}$ & $\begin{array}{l}0.64 \\
0.50 \\
1.00 \\
0.84 \\
0.50 \\
0.49\end{array}$ \\
\hline $\begin{array}{l}940413 \\
940413 \\
940413 \\
940413 \\
940413 \\
940413 \\
940413\end{array}$ & $\begin{array}{l}0159 \\
0503 \\
0803 \\
1102 \\
1358 \\
1703 \\
2003\end{array}$ & $\begin{array}{l}1.51 \\
1.53 \\
1.72 \\
1.76 \\
1.93 \\
2.10 \\
2.37\end{array}$ & $\begin{array}{l}0.083 \\
0.083 \\
0.083 \\
0.083 \\
0.132 \\
0.132 \\
0.123\end{array}$ & $\begin{array}{r}12.0 \\
12.0 \\
12.0 \\
12.0 \\
7.6 \\
7.6 \\
8.2\end{array}$ & $\begin{array}{l}58 \\
60 \\
66 \\
58 \\
56 \\
56 \\
68\end{array}$ & $\begin{array}{l}68 \\
69 \\
63 \\
61 \\
58 \\
54 \\
56\end{array}$ & $\begin{array}{l}0.67 \\
0.71 \\
0.64 \\
0.61 \\
0.56 \\
0.55 \\
0.56\end{array}$ & $\begin{array}{l}2.01 \\
1.89 \\
1.75 \\
1.74 \\
2.02 \\
1.24 \\
0.87\end{array}$ & $\begin{array}{l}4.41 \\
3.97 \\
4.90 \\
5.23 \\
6.34 \\
6.01 \\
5.27\end{array}$ & $\begin{array}{l}34 \\
39 \\
34 \\
32 \\
26 \\
34 \\
38\end{array}$ & $\begin{array}{r}0.26 \\
0.41 \\
-0.10 \\
0.10 \\
0.06 \\
-0.11 \\
-0.23\end{array}$ \\
\hline $\begin{array}{l}940414 \\
940414 \\
940414 \\
940414 \\
940414\end{array}$ & $\begin{array}{l}0203 \\
1103 \\
1406 \\
1659 \\
2006\end{array}$ & $\begin{array}{l}2.25 \\
2.57 \\
2.36 \\
2.14 \\
2.14\end{array}$ & $\begin{array}{l}0.083 \\
0.093 \\
0.083 \\
0.083 \\
0.093\end{array}$ & $\begin{array}{l}12.0 \\
10.7 \\
12.0 \\
12.0 \\
10.7\end{array}$ & $\begin{array}{l}52 \\
52 \\
48 \\
52 \\
52\end{array}$ & $\begin{array}{l}56 \\
57 \\
57 \\
55 \\
54\end{array}$ & $\begin{array}{l}0.55 \\
0.52 \\
0.56 \\
0.61 \\
0.63\end{array}$ & $\begin{array}{l}1.70 \\
1.62 \\
2.30 \\
2.23 \\
1.89\end{array}$ & $\begin{array}{l}6.18 \\
6.72 \\
6.50 \\
5.87 \\
5.31\end{array}$ & $\begin{array}{l}28 \\
30 \\
28 \\
31 \\
38\end{array}$ & $\begin{array}{l}0.10 \\
0.11 \\
0.41 \\
0.21 \\
0.07\end{array}$ \\
\hline $\begin{array}{l}940415 \\
940415 \\
940415 \\
940415 \\
940415 \\
940415\end{array}$ & $\begin{array}{l}0156 \\
0506 \\
0806 \\
1106 \\
2006 \\
2306\end{array}$ & $\begin{array}{l}2.02 \\
1.90 \\
1.74 \\
1.83 \\
1.36 \\
1.50\end{array}$ & $\begin{array}{l}0.093 \\
0.103 \\
0.093 \\
0.093 \\
0.093 \\
0.093\end{array}$ & $\begin{array}{r}10.7 \\
9.7 \\
10.7 \\
10.7 \\
10.7 \\
10.7\end{array}$ & $\begin{array}{l}48 \\
44 \\
64 \\
58 \\
48 \\
58\end{array}$ & $\begin{array}{l}58 \\
63 \\
70 \\
68 \\
73 \\
71\end{array}$ & $\begin{array}{l}0.60 \\
0.66 \\
0.71 \\
0.71 \\
0.83 \\
0.80\end{array}$ & $\begin{array}{l}2.65 \\
2.03 \\
1.91 \\
2.25 \\
1.70 \\
2.01\end{array}$ & $\begin{array}{l}5.95 \\
4.59 \\
4.00 \\
4.12 \\
2.98 \\
3.38\end{array}$ & $\begin{array}{l}27 \\
38 \\
36 \\
34 \\
58 \\
42\end{array}$ & $\begin{array}{l}0.31 \\
0.52 \\
0.19 \\
0.30 \\
0.89 \\
0.83\end{array}$ \\
\hline $\begin{array}{l}940416 \\
940416 \\
940416 \\
940416 \\
940416 \\
940416\end{array}$ & $\begin{array}{l}0206 \\
0506 \\
0803 \\
1106 \\
1706 \\
2007\end{array}$ & $\begin{array}{l}1.57 \\
1.46 \\
1.37 \\
1.51 \\
1.52 \\
1.54\end{array}$ & $\begin{array}{l}0.074 \\
0.074 \\
0.074 \\
0.074 \\
0.083 \\
0.083\end{array}$ & $\begin{array}{l}13.6 \\
13.6 \\
13.6 \\
13.6 \\
12.0 \\
12.0\end{array}$ & $\begin{array}{l}48 \\
50 \\
52 \\
46 \\
54 \\
66\end{array}$ & $\begin{array}{l}68 \\
69 \\
73 \\
69 \\
70 \\
77\end{array}$ & $\begin{array}{l}0.76 \\
0.78 \\
0.78 \\
0.81 \\
0.75 \\
0.75\end{array}$ & $\begin{array}{l}2.47 \\
2.43 \\
2.15 \\
2.16 \\
2.37 \\
1.72\end{array}$ & $\begin{array}{l}3.65 \\
3.59 \\
3.38 \\
3.26 \\
3.79 \\
3.36\end{array}$ & $\begin{array}{l}36 \\
41 \\
45 \\
48 \\
36 \\
40\end{array}$ & $\begin{array}{l}1.05 \\
1.20 \\
0.99 \\
0.97 \\
1.01 \\
0.56\end{array}$ \\
\hline $\begin{array}{l}940417 \\
940417 \\
940417 \\
940417 \\
940417\end{array}$ & $\begin{array}{l}0204 \\
0751 \\
1103 \\
1403 \\
1701\end{array}$ & $\begin{array}{l}1.49 \\
1.38 \\
1.32 \\
1.28 \\
1.28\end{array}$ & $\begin{array}{l}0.083 \\
0.083 \\
0.083 \\
0.083 \\
0.083\end{array}$ & $\begin{array}{l}12.0 \\
12.0 \\
12.0 \\
12.0 \\
12.0\end{array}$ & $\begin{array}{l}50 \\
50 \\
58 \\
58 \\
60\end{array}$ & $\begin{array}{l}70 \\
76 \\
77 \\
78 \\
77\end{array}$ & $\begin{array}{l}0.77 \\
0.81 \\
0.75 \\
0.74 \\
0.76\end{array}$ & $\begin{array}{l}2.25 \\
1.69 \\
1.79 \\
1.68 \\
1.63\end{array}$ & $\begin{array}{l}3.68 \\
2.92 \\
3.20 \\
3.26 \\
3.15\end{array}$ & $\begin{array}{l}39 \\
55 \\
45 \\
44 \\
48\end{array}$ & $\begin{array}{l}0.63 \\
0.76 \\
1.08 \\
0.93 \\
1.02\end{array}$ \\
\hline $\begin{array}{l}940418 \\
940418 \\
940418 \\
940418 \\
940418\end{array}$ & $\begin{array}{l}0501 \\
0808 \\
1148 \\
1407 \\
2005\end{array}$ & $\begin{array}{l}0.92 \\
0.92 \\
0.93 \\
0.97 \\
1.53\end{array}$ & $\begin{array}{l}0.083 \\
0.074 \\
0.093 \\
0.083 \\
0.162\end{array}$ & $\begin{array}{r}12.0 \\
13.6 \\
10.7 \\
12.0 \\
6.2\end{array}$ & $\begin{array}{l}48 \\
60 \\
68 \\
60 \\
62\end{array}$ & $\begin{array}{l}90 \\
92 \\
87 \\
83 \\
66\end{array}$ & $\begin{array}{l}0.90 \\
0.88 \\
0.82 \\
0.83 \\
0.70\end{array}$ & $\begin{array}{l}0.83 \\
0.64 \\
1.11 \\
1.14 \\
1.22\end{array}$ & $\begin{array}{l}1.92 \\
1.99 \\
2.54 \\
2.56 \\
3.69\end{array}$ & $\begin{array}{l}99 \\
93 \\
73 \\
71 \\
41\end{array}$ & $\begin{array}{r}1.33 \\
0.96 \\
1.15 \\
1.14 \\
-0.03\end{array}$ \\
\hline $\begin{array}{l}940419 \\
940419 \\
940419 \\
940419\end{array}$ & $\begin{array}{l}1012 \\
1401 \\
1705 \\
2002\end{array}$ & $\begin{array}{l}2.96 \\
3.09 \\
3.06 \\
2.97\end{array}$ & $\begin{array}{l}0.074 \\
0.083 \\
0.083 \\
0.083\end{array}$ & $\begin{array}{l}13.6 \\
12.0 \\
12.0 \\
12.0\end{array}$ & $\begin{array}{l}70 \\
74 \\
64 \\
66\end{array}$ & $\begin{array}{l}59 \\
62 \\
60 \\
60\end{array}$ & $\begin{array}{l}0.58 \\
0.54 \\
0.53 \\
0.51\end{array}$ & $\begin{array}{l}0.62 \\
0.34 \\
0.71 \\
0.73\end{array}$ & $\begin{array}{l}4.72 \\
4.61 \\
5.56 \\
5.52\end{array}$ & $\begin{array}{l}37 \\
36 \\
32 \\
32\end{array}$ & $\begin{array}{l}-0.46 \\
-0.39 \\
-0.15 \\
-0.14\end{array}$ \\
\hline $\begin{array}{l}940420 \\
940420 \\
940420 \\
940420 \\
940420 \\
940420\end{array}$ & $\begin{array}{l}0203 \\
0503 \\
0803 \\
1406 \\
1710 \\
2006\end{array}$ & $\begin{array}{l}2.69 \\
2.64 \\
2.37 \\
2.34 \\
2.24 \\
2.14\end{array}$ & $\begin{array}{l}0.132 \\
0.123 \\
0.132 \\
0.132 \\
0.132 \\
0.132\end{array}$ & $\begin{array}{l}7.6 \\
8.2 \\
7.6 \\
7.6 \\
7.6 \\
7.6\end{array}$ & $\begin{array}{l}60 \\
64 \\
60 \\
60 \\
64 \\
58\end{array}$ & $\begin{array}{l}58 \\
64 \\
65 \\
61 \\
63 \\
63\end{array}$ & $\begin{array}{l}0.51 \\
0.49 \\
0.53 \\
0.52 \\
0.52 \\
0.50\end{array}$ & $\begin{array}{l}1.23 \\
1.21 \\
1.73 \\
1.34 \\
1.02 \\
1.11\end{array}$ & $\begin{array}{l}5.91 \\
6.07 \\
6.30 \\
6.22 \\
5.90 \\
5.70\end{array}$ & $\begin{array}{l}30 \\
29 \\
30 \\
30 \\
28 \\
29\end{array}$ & $\begin{array}{r}-0.17 \\
-0.10 \\
0.20 \\
-0.01 \\
-0.11 \\
0.22\end{array}$ \\
\hline 940421 & 0203 & 1.89 & 0.132 & 7.6 & 64 & 64 & 0.54 & 0.98 & 5.19 & 33 & -0.02 \\
\hline
\end{tabular}

(Sheet 13 of 45) 


\begin{tabular}{|c|c|c|c|c|c|c|c|c|c|c|c|}
\hline Date & $\begin{array}{l}\text { Time } \\
\text { GMT }\end{array}$ & $\begin{array}{l}H_{m o} \\
\mathrm{~m}\end{array}$ & $\begin{array}{l}f_{p} \\
H_{z}\end{array}$ & $\begin{array}{l}T_{p} \\
\text { sec }\end{array}$ & $\begin{array}{l}\theta_{p} \\
\text { deg }\end{array}$ & $\begin{array}{l}\theta_{0} \\
\text { deg }\end{array}$ & 0 & $Y$ & $\delta$ & $\begin{array}{c}\Delta \theta \\
\operatorname{deg}\end{array}$ & A \\
\hline $\begin{array}{l}940421 \\
940421 \\
940421 \\
940421 \\
940421 \\
940421\end{array}$ & $\begin{array}{l}0503 \\
0836 \\
1106 \\
1406 \\
1711 \\
2006\end{array}$ & $\begin{array}{l}1.86 \\
1.63 \\
1.34 \\
1.17 \\
1.19 \\
1.40\end{array}$ & $\begin{array}{l}0.142 \\
0.152 \\
0.152 \\
0.162 \\
0.162 \\
0.162\end{array}$ & $\begin{array}{l}7.0 \\
6.6 \\
6.6 \\
6.2 \\
6.2 \\
6.2\end{array}$ & $\begin{array}{l}62 \\
58 \\
56 \\
62 \\
66 \\
62\end{array}$ & $\begin{array}{l}62 \\
64 \\
66 \\
71 \\
72 \\
66\end{array}$ & $\begin{array}{l}0.55 \\
0.57 \\
0.64 \\
0.67 \\
0.66 \\
0.61\end{array}$ & $\begin{array}{l}1.05 \\
1.58 \\
1.55 \\
1.37 \\
1.35 \\
1.33\end{array}$ & $\begin{array}{l}5.22 \\
5.14 \\
4.18 \\
3.92 \\
4.05 \\
4.57\end{array}$ & $\begin{array}{l}34 \\
32 \\
39 \\
40 \\
37 \\
34\end{array}$ & $\begin{array}{l}0.03 \\
0.28 \\
0.48 \\
0.30 \\
0.36 \\
0.32\end{array}$ \\
\hline $\begin{array}{l}940422 \\
940422 \\
940422 \\
940422 \\
940422 \\
940422 \\
940422\end{array}$ & $\begin{array}{l}0204 \\
0506 \\
0806 \\
1106 \\
1406 \\
1702 \\
2002\end{array}$ & $\begin{array}{l}2.45 \\
2.33 \\
2.42 \\
2.48 \\
2.61 \\
2.36 \\
2.16\end{array}$ & $\begin{array}{l}0.123 \\
0.123 \\
0.074 \\
0.074 \\
0.074 \\
0.074 \\
0.074\end{array}$ & $\begin{array}{r}8.2 \\
8.2 \\
13.6 \\
13.6 \\
13.6 \\
13.6 \\
13.6\end{array}$ & $\begin{array}{l}66 \\
76 \\
74 \\
64 \\
76 \\
70 \\
64\end{array}$ & $\begin{array}{l}61 \\
66 \\
66 \\
66 \\
70 \\
68 \\
69\end{array}$ & $\begin{array}{l}0.48 \\
0.50 \\
0.47 \\
0.46 \\
0.44 \\
0.49 \\
0.50\end{array}$ & $\begin{array}{l}0.92 \\
1.08 \\
1.06 \\
1.28 \\
0.77 \\
0.84 \\
1.29\end{array}$ & $\begin{array}{l}5.74 \\
5.79 \\
6.63 \\
7.35 \\
7.00 \\
6.08 \\
6.74\end{array}$ & $\begin{array}{l}33 \\
32 \\
27 \\
25 \\
25 \\
27 \\
25\end{array}$ & $\begin{array}{r}-0.24 \\
-0.18 \\
-0.32 \\
0.03 \\
-0.17 \\
-0.07 \\
0.09\end{array}$ \\
\hline $\begin{array}{l}940423 \\
940423 \\
940423 \\
940423 \\
940423 \\
940423 \\
940423\end{array}$ & $\begin{array}{l}0202 \\
0502 \\
0802 \\
1102 \\
1402 \\
1702 \\
2002\end{array}$ & $\begin{array}{l}2.48 \\
2.45 \\
2.34 \\
2.12 \\
1.85 \\
1.69 \\
1.54\end{array}$ & $\begin{array}{l}0.083 \\
0.083 \\
0.083 \\
0.083 \\
0.083 \\
0.083 \\
0.083\end{array}$ & $\begin{array}{l}12.0 \\
12.0 \\
12.0 \\
12.0 \\
12.0 \\
12.0 \\
12.0\end{array}$ & $\begin{array}{l}68 \\
68 \\
66 \\
62 \\
68 \\
74 \\
68\end{array}$ & $\begin{array}{l}71 \\
70 \\
69 \\
69 \\
72 \\
75 \\
76\end{array}$ & $\begin{array}{l}0.48 \\
0.45 \\
0.45 \\
0.52 \\
0.55 \\
0.61 \\
0.59\end{array}$ & $\begin{array}{l}1.02 \\
1.17 \\
1.84 \\
1.75 \\
1.47 \\
1.26 \\
1.93\end{array}$ & $\begin{array}{l}6.44 \\
7.88 \\
8.49 \\
6.61 \\
5.57 \\
4.89 \\
5.36\end{array}$ & $\begin{array}{l}25 \\
20 \\
18 \\
24 \\
27 \\
25 \\
23\end{array}$ & $\begin{array}{l}0.03 \\
0.08 \\
0.10 \\
0.32 \\
0.12 \\
0.01 \\
0.46\end{array}$ \\
\hline $\begin{array}{l}940424 \\
940424 \\
940424 \\
940424 \\
940424 \\
940424 \\
940424\end{array}$ & $\begin{array}{l}0202 \\
0502 \\
0802 \\
1102 \\
1359 \\
1702 \\
2000\end{array}$ & $\begin{array}{l}2.17 \\
2.25 \\
2.35 \\
2.53 \\
2.73 \\
2.79 \\
2.68\end{array}$ & $\begin{array}{l}0.083 \\
0.093 \\
0.093 \\
0.093 \\
0.093 \\
0.093 \\
0.093\end{array}$ & $\begin{array}{l}12.0 \\
10.7 \\
10.7 \\
10.7 \\
10.7 \\
10.7 \\
10.7\end{array}$ & $\begin{array}{l}74 \\
70 \\
56 \\
58 \\
78 \\
52 \\
56\end{array}$ & $\begin{array}{l}70 \\
68 \\
66 \\
68 \\
69 \\
66 \\
67\end{array}$ & $\begin{array}{l}0.51 \\
0.52 \\
0.54 \\
0.52 \\
0.53 \\
0.56 \\
0.58\end{array}$ & $\begin{array}{l}0.95 \\
0.78 \\
1.12 \\
1.10 \\
0.89 \\
0.87 \\
1.05\end{array}$ & $\begin{array}{l}5.57 \\
4.98 \\
5.08 \\
5.21 \\
4.84 \\
4.39 \\
4.76\end{array}$ & $\begin{array}{l}30 \\
33 \\
35 \\
33 \\
36 \\
44 \\
37\end{array}$ & $\begin{array}{r}-0.20 \\
-0.14 \\
0.14 \\
0.16 \\
-0.08 \\
0.18 \\
0.15\end{array}$ \\
\hline $\begin{array}{l}940425 \\
940425 \\
940425 \\
940425 \\
940425 \\
940425 \\
940425 \\
940425\end{array}$ & $\begin{array}{l}0159 \\
0459 \\
0803 \\
1102 \\
1403 \\
1703 \\
2002 \\
2302\end{array}$ & $\begin{array}{l}2.33 \\
2.20 \\
1.99 \\
2.15 \\
2.28 \\
2.27 \\
2.24 \\
2.55\end{array}$ & $\begin{array}{l}0.093 \\
0.064 \\
0.093 \\
0.074 \\
0.074 \\
0.113 \\
0.113 \\
0.093\end{array}$ & $\begin{array}{r}10.7 \\
15.6 \\
10.7 \\
13.6 \\
13.6 \\
8.9 \\
8.9 \\
10.7\end{array}$ & $\begin{array}{l}58 \\
56 \\
64 \\
60 \\
60 \\
76 \\
70 \\
58\end{array}$ & $\begin{array}{l}70 \\
73 \\
77 \\
76 \\
77 \\
78 \\
75 \\
70\end{array}$ & $\begin{array}{l}0.55 \\
0.63 \\
0.69 \\
0.63 \\
0.63 \\
0.60 \\
0.60 \\
0.57\end{array}$ & $\begin{array}{l}1.40 \\
1.48 \\
1.31 \\
1.25 \\
1.02 \\
1.01 \\
1.32 \\
1.63\end{array}$ & $\begin{array}{l}5.15 \\
4.20 \\
3.69 \\
3.82 \\
3.71 \\
4.27 \\
4.73 \\
5.07\end{array}$ & $\begin{array}{l}34 \\
40 \\
44 \\
40 \\
45 \\
34 \\
28 \\
30\end{array}$ & $\begin{array}{l}0.38 \\
0.58 \\
0.60 \\
0.65 \\
0.52 \\
0.06 \\
0.20 \\
0.33\end{array}$ \\
\hline $\begin{array}{l}940426 \\
940426 \\
940426 \\
940426 \\
940426 \\
940426 \\
940426\end{array}$ & $\begin{array}{l}0202 \\
0502 \\
0802 \\
1102 \\
1403 \\
1703 \\
2003\end{array}$ & $\begin{array}{l}3.61 \\
4.06 \\
3.95 \\
3.83 \\
4.03 \\
3.87 \\
3.46\end{array}$ & $\begin{array}{l}0.074 \\
0.074 \\
0.064 \\
0.074 \\
0.064 \\
0.064 \\
0.074\end{array}$ & $\begin{array}{l}13.6 \\
13.6 \\
15.6 \\
13.6 \\
15.6 \\
15.6 \\
13.6\end{array}$ & $\begin{array}{l}54 \\
52 \\
68 \\
58 \\
56 \\
58 \\
52\end{array}$ & $\begin{array}{r}65 \\
64 \\
67 \\
65 \\
65 \\
67 \\
65\end{array}$ & $\begin{array}{l}0.49 \\
0.50 \\
0.51 \\
0.49 \\
0.50 \\
0.54 \\
0.54\end{array}$ & $\begin{array}{l}1.44 \\
1.38 \\
1.16 \\
1.35 \\
1.15 \\
1.36 \\
1.31\end{array}$ & $\begin{array}{l}5.95 \\
5.73 \\
6.12 \\
6.69 \\
6.35 \\
5.58 \\
5.64\end{array}$ & $\begin{array}{l}29 \\
31 \\
27 \\
25 \\
29 \\
30 \\
32\end{array}$ & $\begin{array}{r}0.66 \\
0.65 \\
-0.01 \\
0.20 \\
0.31 \\
0.34 \\
0.12\end{array}$ \\
\hline $\begin{array}{l}940427 \\
940427 \\
940427 \\
940427 \\
940427 \\
940427\end{array}$ & $\begin{array}{l}0203 \\
0503 \\
1010 \\
1354 \\
1652 \\
1952\end{array}$ & $\begin{array}{l}3.62 \\
3.72 \\
3.22 \\
3.18 \\
3.27 \\
3.08\end{array}$ & $\begin{array}{l}0.074 \\
0.083 \\
0.083 \\
0.083 \\
0.083 \\
0.093\end{array}$ & $\begin{array}{l}13.6 \\
12.0 \\
12.0 \\
12.0 \\
12.0 \\
10.7\end{array}$ & $\begin{array}{l}56 \\
64 \\
58 \\
54 \\
48 \\
56\end{array}$ & $\begin{array}{l}64 \\
67 \\
62 \\
60 \\
62 \\
59\end{array}$ & $\begin{array}{l}0.50 \\
0.49 \\
0.54 \\
0.55 \\
0.59 \\
0.57\end{array}$ & $\begin{array}{l}1.32 \\
1.09 \\
1.45 \\
1.36 \\
1.18 \\
1.43\end{array}$ & $\begin{array}{l}6.12 \\
6.19 \\
5.97 \\
5.20 \\
4.40 \\
5.22\end{array}$ & $\begin{array}{l}28 \\
29 \\
31 \\
35 \\
44 \\
35\end{array}$ & $\begin{array}{l}0.37 \\
0.08 \\
0.18 \\
0.28 \\
0.85 \\
0.05\end{array}$ \\
\hline $\begin{array}{l}940428 \\
940428 \\
940428\end{array}$ & $\begin{array}{l}0152 \\
0453 \\
0752\end{array}$ & $\begin{array}{l}3.07 \\
3.01 \\
2.46\end{array}$ & $\begin{array}{l}0.093 \\
0.093 \\
0.093\end{array}$ & $\begin{array}{l}10.7 \\
10.7 \\
10.7\end{array}$ & $\begin{array}{l}42 \\
56 \\
54\end{array}$ & $\begin{array}{l}57 \\
64 \\
65\end{array}$ & $\begin{array}{l}0.53 \\
0.55 \\
0.58\end{array}$ & $\begin{array}{l}1.37 \\
1.27 \\
1.18\end{array}$ & $\begin{array}{l}5.50 \\
5.04 \\
4.87\end{array}$ & $\begin{array}{l}35 \\
37 \\
40\end{array}$ & $\begin{array}{l}0.42 \\
0.54 \\
0.47\end{array}$ \\
\hline 940429 & 0158 & 1.46 & 0.103 & 9.7 & 58 & 66 & 0.68 & 1.34 & 3.77 & 45 & 0.31 \\
\hline
\end{tabular}

(Sheet 14 of 45 ) 


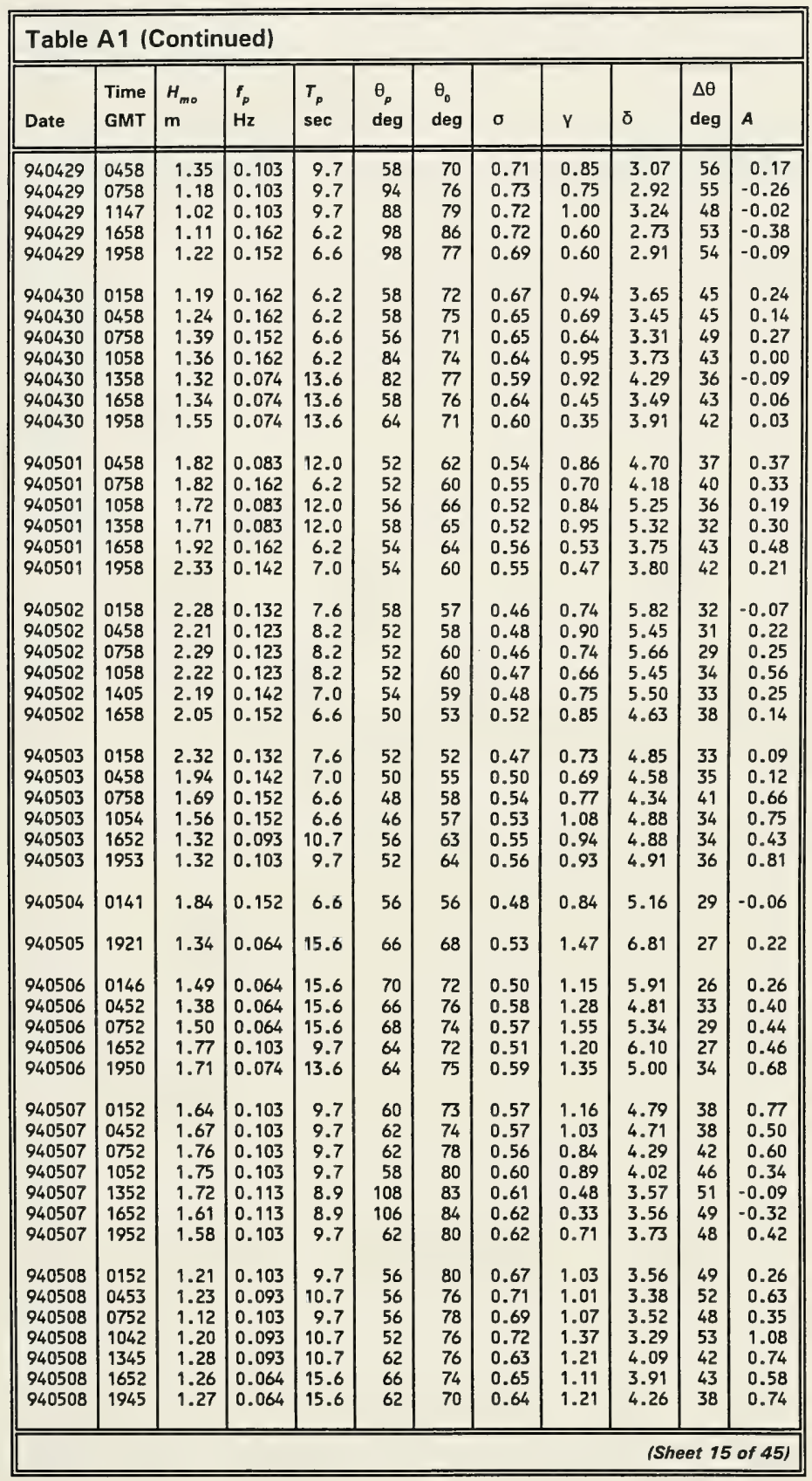




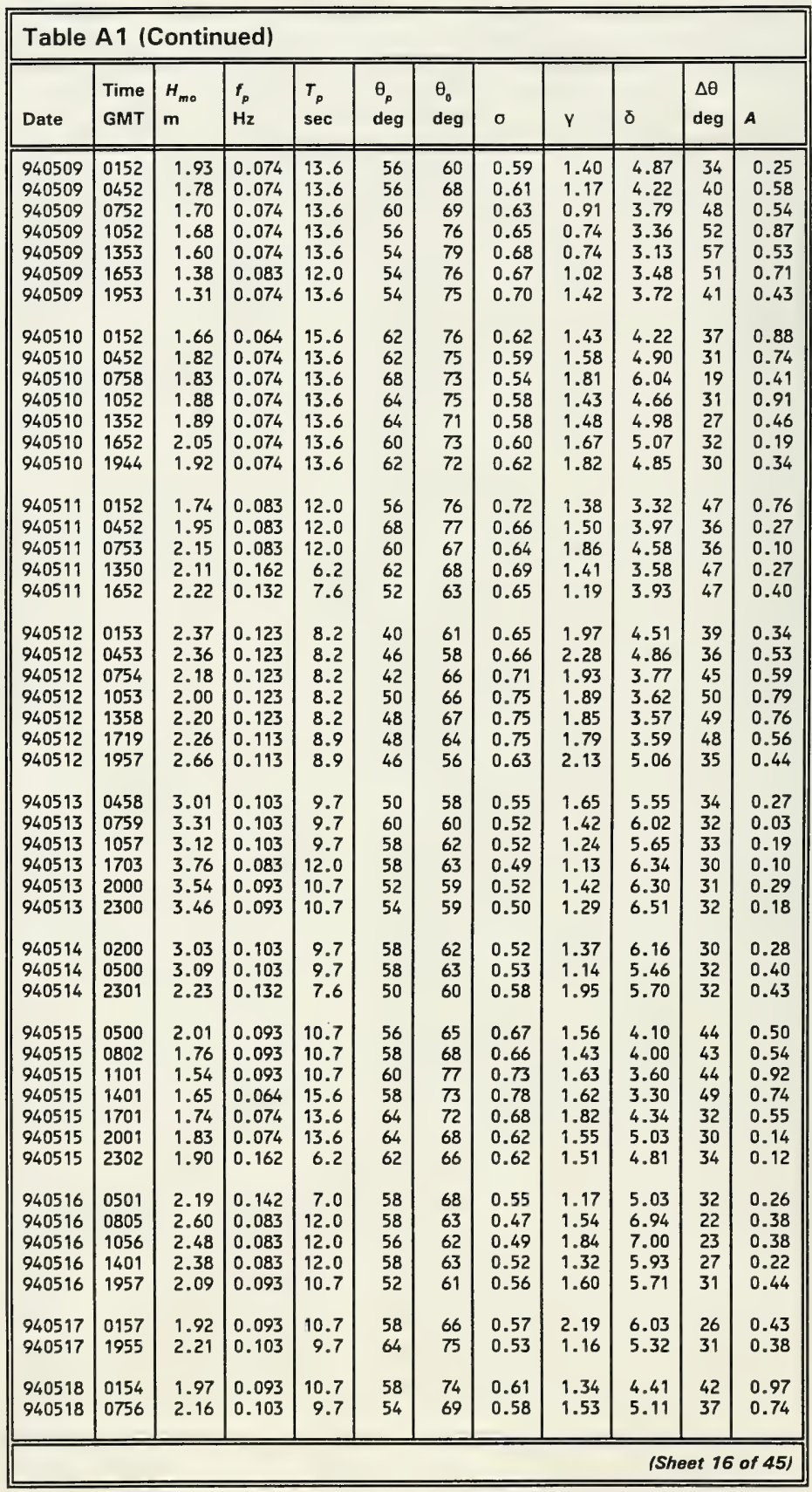




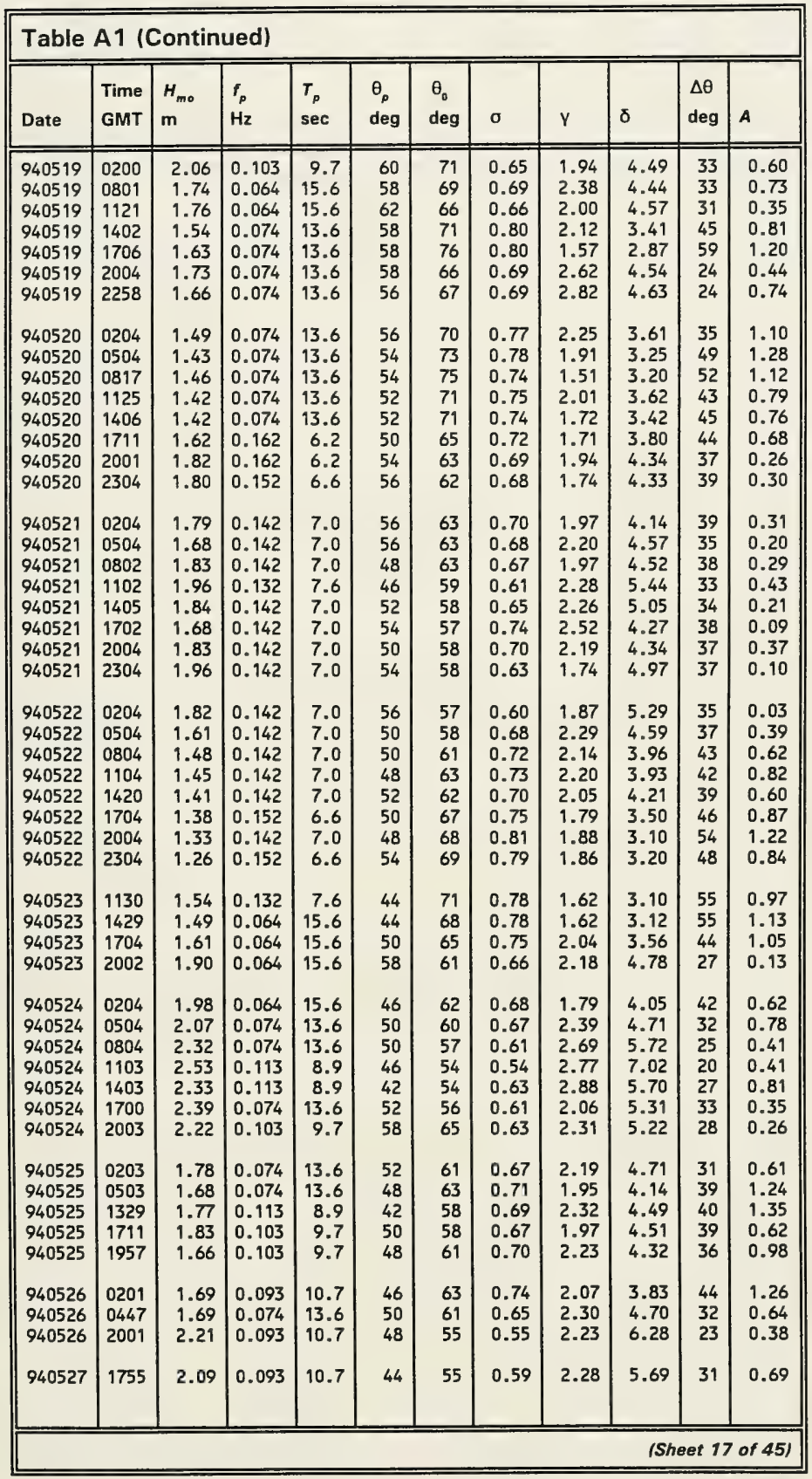




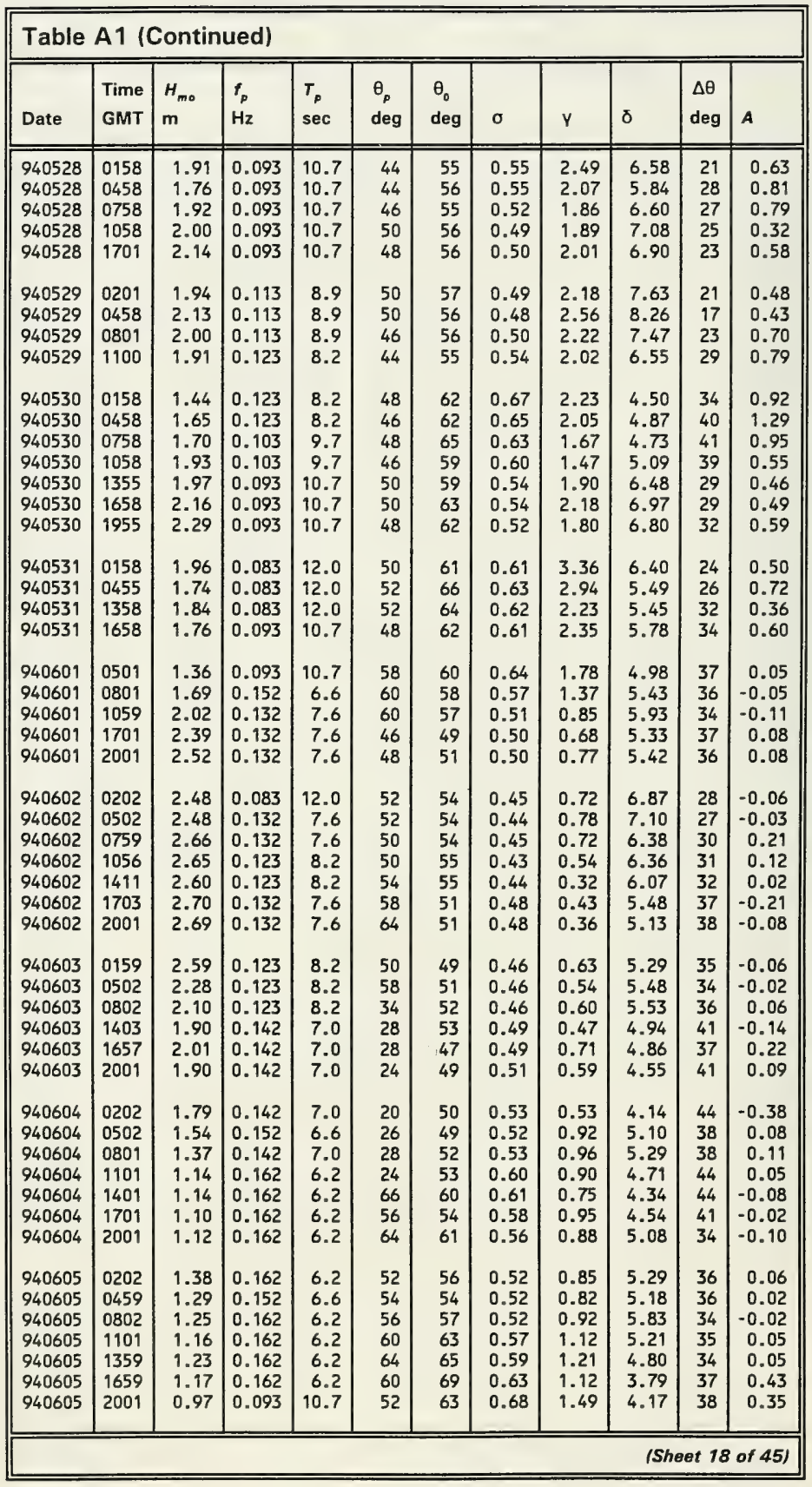




\begin{tabular}{|c|c|c|c|c|c|c|c|c|c|c|c|}
\hline \multicolumn{12}{|c|}{ Table A1 (Continued) } \\
\hline Date & $\begin{array}{l}\text { Time } \\
\text { GMT }\end{array}$ & $\begin{array}{l}H_{m o} \\
m\end{array}$ & $\begin{array}{l}f_{p} \\
\mathrm{~Hz}\end{array}$ & $\begin{array}{l}T_{p} \\
\sec \end{array}$ & $\begin{array}{l}\theta_{p} \\
\text { deg }\end{array}$ & $\begin{array}{l}\theta_{0} \\
\text { deg }\end{array}$ & $\sigma$ & $r$ & $\delta$ & $\begin{array}{l}\Delta \theta \\
\text { deg }\end{array}$ & $A$ \\
\hline 940606 & 0202 & 0.81 & 0.103 & 9.7 & 68 & 74 & 0.68 & 1.10 & 3.71 & 43 & 0.28 \\
\hline 940606 & 0502 & 0.77 & 0.103 & 9.7 & 64 & 75 & 0.72 & 1.04 & 3.11 & 50 & 0.48 \\
\hline 940606 & 0756 & 0.75 & 0.113 & 8.9 & 66 & 74 & 0.77 & 0.91 & 2.79 & 61 & 0.39 \\
\hline 940606 & 1103 & 0.77 & 0.113 & 8.9 & 56 & 77 & 0.78 & 0.70 & 2.53 & 69 & 0.57 \\
\hline 940606 & 1359 & 1.19 & 0.162 & 6.2 & 62 & 69 & 0.64 & 0.63 & 3.51 & 48 & 0.17 \\
\hline 940606 & 1703 & 1.90 & 0.162 & 6.2 & 56 & 59 & 0.54 & 0.64 & 4.45 & 42 & 0.06 \\
\hline 940606 & 1959 & 2.08 & 0.142 & 7.0 & 28 & 52 & 0.51 & 0.86 & 4.77 & 39 & 0.17 \\
\hline 940607 & 0201 & 2.79 & 0.123 & 8.2 & 56 & 53 & 0.46 & 0.81 & 4.81 & 33 & 0.03 \\
\hline 940607 & 0502 & 2.93 & 0.123 & 8.2 & 56 & 58 & 0.42 & 0.52 & 5.22 & 30 & 0.09 \\
\hline 940607 & 0802 & 2.84 & 0.113 & 8.9 & 54 & 59 & 0.44 & 0.36 & 4.89 & 35 & 0.06 \\
\hline 940607 & 1103 & 2.70 & 0.123 & 8.2 & 52 & 59 & 0.46 & 0.73 & 5.13 & 32 & 0.15 \\
\hline 940607 & 1403 & 2.70 & 0.123 & 8.2 & 50 & 59 & 0.48 & 0.66 & 5.19 & 34 & 0.33 \\
\hline 940607 & 1701 & 3.03 & 0.113 & 8.9 & 50 & 60 & 0.45 & 0.48 & 4.86 & 34 & 0.21 \\
\hline 940607 & 2001 & 3.05 & 0.103 & 9.7 & 46 & 56 & 0.43 & 0.65 & 5.06 & 33 & 0.37 \\
\hline 940608 & 0202 & 3.22 & 0.103 & 9.7 & 50 & 56 & 0.39 & 0.51 & 5.88 & 28 & 0.25 \\
\hline 940608 & 0502 & 2.81 & 0.113 & 8.9 & 52 & 56 & 0.43 & 0.61 & 5.65 & 32 & 0.16 \\
\hline 940608 & 0802 & 2.40 & 0.113 & 8.9 & 48 & 56 & 0.45 & 0.65 & 6.06 & 33 & 0.12 \\
\hline 940608 & 1101 & 2.23 & 0.113 & 8.9 & 54 & 61 & 0.45 & 1.02 & 6.41 & 26 & 0.16 \\
\hline 940608 & 1359 & 2.31 & 0.113 & 8.9 & 56 & 64 & 0.50 & 0.62 & 5.38 & 34 & 0.13 \\
\hline 940608 & 1701 & 2.22 & 0.123 & 8.2 & 46 & 61 & 0.51 & 0.60 & 4.58 & 39 & 0.46 \\
\hline 940608 & 2001 & 2.15 & 0.123 & 8.2 & 64 & 65 & 0.48 & 0.38 & 5.25 & 34 & -0.02 \\
\hline 940609 & 0201 & 2.16 & 0.113 & 8.9 & 50 & 60 & 0.46 & 1.14 & 6.77 & 29 & 0.40 \\
\hline 940609 & 0459 & 2.21 & 0.113 & 8.9 & 56 & 63 & 0.48 & 1.02 & 6.75 & 29 & 0.19 \\
\hline 940609 & 0801 & 2.07 & 0.113 & 8.9 & 46 & 62 & 0.50 & 1.33 & 6.22 & 33 & 0.31 \\
\hline 940609 & 1402 & 2.09 & 0.123 & 8.2 & 62 & 71 & 0.55 & 0.76 & 4.70 & 39 & 0.11 \\
\hline 940609 & 1701 & 1.85 & 0.123 & 8.2 & 50 & 69 & 0.57 & 1.16 & 4.67 & 39 & 0.05 \\
\hline 940609 & 2003 & 1.69 & 0.113 & 8.9 & 70 & 68 & 0.59 & 1.25 & 4.94 & 37 & -0.15 \\
\hline 940610 & 0201 & 1.39 & 0.113 & 8.9 & 56 & 76 & 0.68 & 1.24 & 3.76 & 43 & 0.28 \\
\hline 940610 & 0502 & 1.49 & 0.113 & 8.9 & 54 & 73 & 0.68 & 1.5 & 3.73 & 40 & 0.47 \\
\hline 940610 & 0801 & 1.42 & 0.113 & 8.9 & 56 & 76 & 0.72 & 1.08 & 3.20 & 46 & 0.14 \\
\hline 940610 & 1059 & 1.37 & 0.074 & 13.6 & 54 & 79 & 0.78 & 1.11 & 2.8 & 53 & 0.59 \\
\hline 940610 & 1701 & 1.51 & 0.083 & 12.0 & 46 & 77 & 0.75 & 1.35 & 3.11 & 50 & 0.10 \\
\hline 940610 & 2002 & 1.43 & 0.074 & 13.6 & 58 & 81 & 0.78 & 1.28 & 2.76 & 59 & 0.83 \\
\hline 940611 & 0202 & 1.32 & 0.074 & 13.6 & 56 & 86 & 0.78 & 1.19 & 2.5 & 78 & 1.33 \\
\hline 940611 & 0501 & 1.36 & 0.083 & 12.0 & 52 & 81 & 0.7 & 1.3 & 2.7 & 62 & 0.87 \\
\hline 940611 & 0759 & 1.34 & 0.083 & 12.0 & 56 & 91 & 0.89 & 0.82 & 2.04 & 95 & 1.04 \\
\hline 940611 & 1101 & 1.32 & 0.083 & 12.0 & 48 & 82 & 0.86 & & & 86 & 1.07 \\
\hline 940611 & 1401 & 1.44 & 0.083 & 12.0 & 52 & 81 & 0.83 & 1.38 & 2.54 & 80 & 1.23 \\
\hline 940611 & 1702 & 1.34 & 0.093 & 10.7 & 52 & 88 & 0.85 & 1.13 & 2.20 & 94 & 1.30 \\
\hline 940611 & 2001 & 1.21 & 0.064 & 15.6 & 62 & 99 & 0.87 & 0.65 & 1.80 & 96 & 1.31 \\
\hline 940612 & 0159 & 1.27 & 0.064 & 15.6 & 56 & 97 & 0.90 & 0.67 & 1.78 & 98 & 1.23 \\
\hline & 0502 & 1.25 & 0.064 & 15.6 & 54 & 87 & 0.87 & 1.16 & 2.22 & 94 & 1.33 \\
\hline 940612 & 0801 & 1.30 & 0.064 & 15.6 & 54 & 84 & 0.86 & 1.2 & 2.28 & 95 & 1.52 \\
\hline 940612 & 1101 & 1.22 & 0.064 & 15.6 & 56 & 89 & 0.87 & 1.15 & 2.18 & 95 & 1.64 \\
\hline 940612 & 1359 & 1.37 & 0.083 & 12.0 & 52 & 76 & 0.78 & 1.77 & 3.10 & 52 & 1.04 \\
\hline 940612 & 1702 & 1.80 & 0.123 & 8.2 & 52 & 71 & 0.71 & 1.90 & 3.61 & 39 & 0.54 \\
\hline 940612 & 2001 & 1.76 & 0.113 & 8.9 & 54 & 67 & 0.66 & 1.97 & 4.35 & 34 & 0.47 \\
\hline & & & & & 44 & 58 & & 2.02 & & 33 & 0.22 \\
\hline 940613 & 0502 & 1.97 & 0.123 & 8.2 & 48 & 60 & 0.57 & 2.0 & 5.79 & 31 & 0.32 \\
\hline 940613 & 0802 & 1.77 & 0.132 & 7.6 & 44 & 61 & 0.62 & 2.39 & 5.26 & 33 & 0.41 \\
\hline & 1102 & 1.56 & 0.132 & 7.6 & 56 & 60 & 0.59 & 2.16 & 5.84 & 30 & 0.04 \\
\hline 940613 & 1403 & 1.53 & 0.132 & 7.6 & 42 & 58 & 0.62 & 2.07 & 5.21 & 34 & 0.36 \\
\hline 940613 & 1703 & 1.87 & 0.142 & 7.0 & 40 & 57 & 0.57 & 1.22 & 5.01 & 39 & 0.04 \\
\hline
\end{tabular}




\begin{tabular}{|c|c|c|c|c|c|c|c|c|c|c|c|}
\hline \multicolumn{12}{|c|}{ Table A1 (Continued) } \\
\hline Date & $\begin{array}{l}\text { Time } \\
\text { GMT }\end{array}$ & $\begin{array}{l}H_{m o} \\
\mathrm{~m}\end{array}$ & $\begin{array}{l}f_{p} \\
\mathrm{~Hz}\end{array}$ & $\begin{array}{l}T_{p} \\
\text { sec }\end{array}$ & $\begin{array}{l}\theta_{p} \\
\text { deg }\end{array}$ & $\begin{array}{l}\theta_{0} \\
\text { deg }\end{array}$ & $\sigma$ & Y & $\delta$ & $\begin{array}{l}\Delta \theta \\
\text { deg }\end{array}$ & A \\
\hline 940613 & 2003 & 1.79 & 0.142 & 7.0 & 34 & 58 & 0.56 & 1.08 & 5.26 & 39 & 0.00 \\
\hline 940614 & 0202 & 2.12 & 0.123 & 8.2 & 44 & 52 & 0.48 & 1.22 & 6.46 & 31 & 0.23 \\
\hline 940614 & 0459 & 2.17 & 0.123 & 8.2 & 50 & 55 & 0.45 & 1.66 & 8.24 & 24 & 0.23 \\
\hline 940614 & 0801 & 2.18 & 0.123 & 8.2 & 48 & 57 & 0.45 & 2.25 & 8.70 & 22 & 0.45 \\
\hline 940614 & 1102 & 2.34 & 0.113 & 8.9 & 48 & 58 & 0.47 & 1.85 & 7.34 & 25 & 0.45 \\
\hline 940614 & 1358 & 2.37 & 0.113 & 8.9 & 48 & 55 & 0.45 & 1.37 & 7.45 & 25 & 0.42 \\
\hline 940614 & 1710 & 2.70 & 0.103 & 9.7 & 48 & 58 & 0.46 & 1.24 & 6.72 & 29 & 0.66 \\
\hline 940614 & 2001 & 3.00 & 0.103 & 9.7 & 48 & 59 & 0.43 & 0.94 & 6.80 & 29 & 0.37 \\
\hline 940615 & 0202 & 3.03 & 0.103 & 9.7 & 48 & 57 & 0.40 & 1.19 & 8.31 & 25 & 0.51 \\
\hline 940615 & 0502 & 3.04 & 0.103 & 9.7 & 50 & 59 & 0.40 & 1.13 & 8.32 & 24 & 0.45 \\
\hline 940615 & 0801 & 3.09 & 0.093 & 10.7 & 52 & 61 & 0.41 & 1.05 & 7.48 & 26 & 0.42 \\
\hline 940615 & 1101 & 3.12 & 0.103 & 9.7 & 54 & 60 & 0.40 & 0.79 & 7.60 & 27 & 0.17 \\
\hline 940615 & 1401 & 2.82 & 0.093 & 10.7 & 52 & 59 & 0.41 & 0.76 & 7.42 & 26 & 0.23 \\
\hline 940615 & 1703 & 2.75 & 0.093 & 10.7 & 50 & 59 & 0.42 & 0.70 & 6.74 & 29 & 0.35 \\
\hline 940615 & 2001 & 2.86 & 0.093 & 10.7 & 52 & 57 & 0.43 & 0.71 & 6.70 & 29 & 0.22 \\
\hline 940616 & 0204 & 2.59 & 0.113 & 8.9 & 48 & 54 & 0.44 & 0.84 & 5.96 & 30 & 0.37 \\
\hline 940616 & 0504 & 2.31 & 0.113 & 8.9 & 52 & 59 & 0.45 & 0.96 & 6.36 & 29 & 0.48 \\
\hline 940616 & 0807 & 2.10 & 0.123 & 8.2 & 52 & 60 & 0.42 & 1.29 & 8.12 & 25 & 0.60 \\
\hline 940616 & 1104 & 2.13 & 0.123 & 8.2 & 52 & 58 & 0.43 & 1.51 & 8.77 & 23 & 0.38 \\
\hline 940616 & 1401 & 1.95 & 0.123 & 8.2 & 48 & 56 & 0.45 & 1.33 & 7.33 & 26 & 0.47 \\
\hline 940616 & 1704 & 2.15 & 0.132 & 7.6 & 50 & 57 & 0.46 & 0.68 & 6.21 & 30 & 0.36 \\
\hline 940616 & 2004 & 2.19 & 0.132 & 7.6 & 54 & 55 & 0.46 & 0.37 & 5.82 & 29 & 0.10 \\
\hline 940617 & 0204 & 2.00 & 0.123 & 8.2 & 48 & 52 & 0.48 & 0.78 & 5.75 & 31 & 0.16 \\
\hline 940617 & 0454 & 1.95 & 0.123 & 8.2 & 48 & 54 & 0.46 & 0.94 & 6.23 & 29 & 0.28 \\
\hline 940617 & 0805 & 2.03 & 0.123 & 8.2 & 50 & 54 & 0.43 & 0.92 & 7.16 & 25 & 0.21 \\
\hline 940617 & 1049 & 2.17 & 0.123 & 8.2 & 54 & 55 & 0.41 & 0.70 & 6.83 & 27 & 0.02 \\
\hline 940617 & 1400 & 1.96 & 0.123 & 8.2 & 52 & 57 & 0.44 & 0.97 & 7.12 & 26 & 0.24 \\
\hline 940617 & 1700 & 1.92 & 0.123 & 8.2 & 52 & 58 & 0.45 & 1.06 & 7.22 & 27 & 0.27 \\
\hline 940617 & 1956 & 2.05 & 0.123 & 8.2 & 54 & 57 & 0.46 & 0.90 & 7.05 & 25 & 0.19 \\
\hline 940618 & 0202 & 2.22 & 0.113 & 8.9 & 52 & 52 & 0.46 & 1.18 & 6.54 & 25 & -0.06 \\
\hline 940618 & 0502 & 2.15 & 0.113 & 8.9 & 50 & 54 & 0.47 & 1.79 & 7.30 & 23 & 0.29 \\
\hline 940618 & 0803 & 2.28 & 0.113 & 8.9 & 48 & 56 & 0.49 & 1.80 & 6.72 & 27 & 0.51 \\
\hline 940618 & 1052 & 2.33 & 0.123 & 8.2 & 48 & 59 & 0.51 & 1.38 & 5.65 & 34 & 0.49 \\
\hline 940618 & 1351 & 2.29 & 0.113 & 8.9 & 46 & 60 & 0.54 & 1.42 & 5.22 & 35 & 0.43 \\
\hline 940618 & 1702 & 1.98 & 0.123 & 8.2 & 48 & 57 & 0.59 & 1.64 & 5.23 & 32 & 0.37 \\
\hline 940618 & 2002 & 1.92 & 0.123 & 8.2 & 42 & 55 & 0.59 & 1.73 & 5.21 & 35 & 0.46 \\
\hline 940619 & 0202 & 1.68 & 0.123 & 8.2 & 46 & 61 & 0.70 & 1.92 & 3.86 & 41 & 0.62 \\
\hline 940619 & 0502 & 1.55 & 0.123 & 8.2 & 46 & 65 & 0.72 & 1.78 & 3.47 & 45 & 0.79 \\
\hline 940619 & 0802 & 1.44 & 0.123 & 8.2 & 48 & 70 & 0.76 & 1.84 & 3.37 & 43 & 0.38 \\
\hline 940619 & 1102 & 1.40 & 0.123 & 8.2 & 44 & 69 & 0.80 & 1.77 & 2.95 & 53 & 0.89 \\
\hline 940619 & 1359 & 1.40 & 0.123 & 8.2 & 42 & 77 & 0.86 & 1.32 & 2.32 & 96 & 1.26 \\
\hline 940619 & 1702 & 1.40 & 0.113 & 8.9 & 44 & 74 & 0.88 & 1.64 & 2.46 & 93 & 1.64 \\
\hline 940619 & 2001 & 1.34 & 0.123 & 8.2 & 46 & 76 & 0.88 & 1.43 & 2.34 & 99 & 1.72 \\
\hline 940620 & 0201 & 1.38 & 0.064 & 15.6 & 42 & 78 & 0.87 & 1.18 & 2.20 & 104 & 1.38 \\
\hline 940620 & 0457 & 1.39 & 0.064 & 15.6 & 42 & 80 & 0.92 & 1.09 & 1.94 & 112 & 1.51 \\
\hline 940620 & 0802 & 1.30 & 0.064 & 15.6 & 40 & 83 & 0.95 & 0.94 & 1.86 & 112 & 1.31 \\
\hline 940620 & 1111 & 1.27 & 0.064 & 15.6 & 38 & 91 & 0.95 & 0.60 & 1.63 & 114 & 0.97 \\
\hline 940620 & 1405 & 1.24 & 0.064 & 15.6 & 170 & 97 & 0.96 & 0.38 & 1.55 & 114 & 0.77 \\
\hline 940620 & 1703 & 1.16 & 0.064 & 15.6 & 44 & 95 & 0.96 & 0.55 & 1.62 & 113 & 0.94 \\
\hline 940620 & 2003 & 1.18 & 0.064 & 15.6 & 42 & 93 & 0.99 & 0.60 & 1.63 & 116 & 0.96 \\
\hline 940621 & 0203 & 1.48 & 0.152 & 6.6 & 48 & 76 & 0.85 & 1.36 & 2.54 & 67 & 0.83 \\
\hline 940621 & 0500 & 1.56 & 0.142 & 7.0 & 44 & 70 & 0.84 & 1.79 & 2.81 & 59 & 1.06 \\
\hline
\end{tabular}




\begin{tabular}{|c|c|c|c|c|c|c|c|c|c|c|c|}
\hline \multicolumn{12}{|c|}{ Table A1 (Continued) } \\
\hline Date & $\begin{array}{l}\text { Time } \\
\text { GMT }\end{array}$ & $\begin{array}{l}H_{m o} \\
\text { m }\end{array}$ & $\begin{array}{l}f_{p} \\
\mathrm{~Hz}\end{array}$ & $\begin{array}{l}T_{p} \\
\text { sec }\end{array}$ & $\begin{array}{l}\theta_{p} \\
\text { deg }\end{array}$ & $\begin{array}{l}\theta_{0} \\
\text { deg }\end{array}$ & $\sigma$ & $\checkmark$ & $\delta$ & $\begin{array}{l}\Delta \theta \\
\operatorname{deg}\end{array}$ & A \\
\hline 940621 & 0801 & 1.59 & 0.152 & 6.6 & 46 & 63 & 0.77 & 2.31 & 3.61 & 43 & 0.95 \\
\hline 940621 & 1102 & 1.67 & 0.142 & 7.0 & 44 & 61 & 0.76 & 2.41 & 3.73 & 45 & 1.28 \\
\hline 940621 & 1402 & 1.57 & 0.142 & 7.0 & 44 & 65 & 0.79 & 2.19 & 3.49 & 48 & 0.84 \\
\hline 940621 & 1716 & 1.68 & 0.142 & 7.0 & 44 & 61 & 0.77 & 2.12 & 3.64 & 47 & 0.61 \\
\hline 940621 & 2000 & 1.57 & 0.142 & 7.0 & 48 & 64 & 0.84 & 2.35 & 3.27 & 44 & 0.80 \\
\hline 940622 & 0200 & 1.53 & 0.132 & 7.6 & 44 & 72 & 0.89 & 1.56 & 2.46 & 86 & 1.37 \\
\hline 940622 & 0500 & 1.62 & 0.132 & 7.6 & 50 & 70 & 0.81 & 1.44 & 2.88 & 55 & 0.43 \\
\hline 940622 & 0814 & 1.64 & 0.132 & 7.6 & 34 & 68 & 0.88 & 1.63 & 2.56 & 69 & 0.87 \\
\hline 940622 & 1114 & 1.53 & 0.142 & 7.0 & 42 & 68 & 0.89 & 1.79 & 2.61 & 77 & 1.44 \\
\hline 940622 & 1704 & 1.39 & 0.152 & 6.6 & 34 & 77 & 0.94 & 1.21 & 2.08 & 113 & 1.19 \\
\hline 940622 & 2004 & 1.30 & 0.142 & 7.0 & 42 & 72 & 0.94 & 1.44 & 2.21 & 110 & 1.56 \\
\hline 940623 & 0201 & 1.55 & 0.132 & 7.6 & 38 & 65 & 0.81 & 1.72 & 3.07 & 53 & 0.46 \\
\hline 940623 & 0504 & 1.70 & 0.132 & 7.6 & 40 & 59 & 0.69 & 2.58 & 4.45 & 38 & 0.72 \\
\hline 940623 & 0808 & 1.73 & 0.132 & 7.6 & 42 & 57 & 0.69 & 2.94 & 4.72 & 32 & 0.70 \\
\hline 940623 & 1108 & 1.72 & 0.123 & 8.2 & 42 & 61 & 0.70 & 2.14 & 4.19 & 41 & 0.66 \\
\hline 940623 & 1406 & 1.75 & 0.132 & 7.6 & 40 & 59 & 0.72 & 2.09 & 3.96 & 46 & 0.88 \\
\hline 940623 & 1712 & 1.93 & 0.132 & 7.6 & 42 & 56 & 0.62 & 1.79 & 4.86 & 40 & 0.66 \\
\hline 940623 & 2004 & 1.96 & 0.132 & 7.6 & 44 & 56 & 0.60 & 2.32 & 5.84 & 33 & 0.50 \\
\hline 940624 & 0201 & 2.09 & 0.123 & 8.2 & 42 & 56 & 0.55 & 1.71 & 5.62 & 37 & 0.66 \\
\hline 940624 & 0501 & 2.10 & 0.113 & 8.9 & 38 & 58 & 0.52 & 1.73 & 6.26 & 35 & 0.25 \\
\hline 940624 & 0801 & 2.05 & 0.123 & 8.2 & 44 & 58 & 0.54 & 1.74 & 6.13 & 34 & 0.51 \\
\hline 940624 & 1103 & 1.96 & 0.123 & 8.2 & 42 & 57 & 0.57 & 2.08 & 5.85 & 33 & 0.25 \\
\hline 940624 & 1406 & 2.04 & 0.123 & 8.2 & 42 & 54 & 0.55 & 2.12 & 6.17 & 30 & 0.36 \\
\hline 940624 & 1702 & 2.38 & 0.123 & 8.2 & 46 & 52 & 0.48 & 1.58 & 7.21 & 28 & 0.18 \\
\hline 940625 & 0150 & 2.30 & 0.113 & 8.9 & 44 & 52 & 0.51 & 2.26 & 7.37 & 25 & 0.49 \\
\hline 940625 & 0502 & 2.25 & 0.113 & 8.9 & 44 & 55 & 0.57 & 2.32 & 5.86 & 31 & 0.44 \\
\hline 940625 & 0802 & 2.14 & 0.113 & 8.9 & 44 & 57 & 0.55 & 2.31 & 6.14 & 29 & 0.58 \\
\hline 940625 & 1101 & 2.12 & 0.113 & 8.9 & 40 & 53 & 0.53 & 2.28 & 6.83 & 28 & 0.64 \\
\hline 940625 & 1401 & 2.44 & 0.113 & 8.9 & 38 & 49 & 0.54 & 2.05 & 6.38 & 31 & 0.45 \\
\hline 940625 & 1658 & 2.79 & 0.113 & 8.9 & 52 & 50 & 0.52 & 1.77 & 6.34 & 31 & 0.00 \\
\hline 940625 & 2004 & 2.65 & 0.113 & 8.9 & 44 & 52 & 0.53 & 2.79 & 7.41 & 24 & 0.32 \\
\hline 940626 & 0202 & 2.49 & 0.113 & 8.9 & 42 & 50 & 0.52 & 2.85 & 7.62 & 23 & 0.53 \\
\hline 940626 & 0502 & 2.19 & 0.113 & 8.9 & 44 & 53 & 0.58 & 2.89 & 6.32 & 26 & 0.41 \\
\hline 940626 & 0804 & 2.21 & 0.113 & 8.9 & 50 & 57 & 0.58 & 2.74 & 6.29 & 25 & 0.34 \\
\hline 940626 & 1102 & 2.02 & 0.113 & 8.9 & 48 & 57 & 0.59 & 2.35 & 5.84 & 30 & 0.36 \\
\hline 940626 & 1401 & 2.04 & 0.123 & 8.2 & 48 & 56 & 0.60 & 2.47 & 5.98 & 29 & 0.20 \\
\hline 940626 & 1704 & 1.85 & 0.123 & 8.2 & 40 & 54 & 0.64 & 2.09 & 5.03 & 36 & 0.35 \\
\hline 940626 & 2004 & 1.76 & 0.113 & 8.9 & 50 & 58 & 0.59 & 2.51 & 5.74 & 26 & 0.37 \\
\hline 940627 & 0204 & 1.61 & 0.123 & 8.2 & 48 & 59 & 0.62 & 2.23 & 5.19 & 30 & 0.49 \\
\hline 940627 & 0504 & 1.33 & 0.123 & 8.2 & 38 & 69 & 0.69 & 1.92 & 4.16 & 44 & 0.58 \\
\hline 940627 & 0823 & 1.28 & 0.132 & 7.6 & 52 & 63 & 0.65 & 2.14 & 4.73 & 34 & 0.47 \\
\hline 940627 & 1410 & 1.32 & 0.123 & 8.2 & 60 & 64 & 0.64 & 1.89 & 4.55 & 37 & 0.15 \\
\hline 940627 & 1704 & 1.38 & 0.132 & 7.6 & 58 & 61 & 0.61 & 1.93 & 5.16 & 33 & 0.10 \\
\hline 940627 & 2004 & 1.46 & 0.142 & 7.0 & 44 & 54 & 0.58 & 1.88 & 5.66 & 32 & 0.32 \\
\hline 940628 & 0205 & 1.43 & 0.132 & 7.6 & 52 & 55 & 0.59 & 2.29 & 6.03 & 26 & 0.05 \\
\hline & 0504 & 1.41 & 0.142 & 7.0 & 56 & 57 & 0.59 & 2.02 & 5.85 & 29 & 0.07 \\
\hline 940628 & 0801 & 1.35 & 0.142 & 7.0 & 46 & 58 & 0.60 & 2.02 & 5.48 & 31 & 0.62 \\
\hline 940628 & 1407 & 1.17 & 0.152 & 6.6 & 48 & 60 & 0.66 & 2.12 & 4.82 & 34 & 0.49 \\
\hline 940628 & 1703 & 1.15 & 0.152 & 6.6 & 44 & 63 & 0.70 & 1.76 & 4.12 & 45 & 0.62 \\
\hline 940628 & 2004 & 1.26 & 0.162 & 6.2 & 46 & 57 & 0.60 & 1.77 & 5.25 & 36 & 0.41 \\
\hline 940629 & 0204 & 1.56 & 0.142 & 7.0 & 52 & 53 & 0.52 & 1.73 & 6.53 & 27 & -0.02 \\
\hline 940629 & 0504 & 2.00 & 0.123 & 8.2 & 46 & 50 & 0.49 & 2.05 & 7.54 & 23 & 0.20 \\
\hline
\end{tabular}




\begin{tabular}{|c|c|c|c|c|c|c|c|c|c|c|c|}
\hline Date & $\begin{array}{l}\text { Time } \\
\text { GMT }\end{array}$ & $\begin{array}{l}H_{m 0} \\
m\end{array}$ & $\begin{array}{l}f_{p} \\
H z\end{array}$ & $\begin{array}{l}T_{p} \\
\text { sec }\end{array}$ & $\begin{array}{l}\theta_{p} \\
\text { deg }\end{array}$ & $\begin{array}{l}\theta_{0} \\
\operatorname{deg}\end{array}$ & $\sigma$ & Y & $\delta$ & $\begin{array}{l}\Delta \theta \\
\operatorname{deg}\end{array}$ & A \\
\hline 940629 & 0813 & 2.31 & 0.123 & 8.2 & 42 & 49 & 0.44 & 1.61 & 7.97 & 24 & 0.48 \\
\hline 940629 & 1107 & 2.31 & 0.113 & 8.9 & 42 & 48 & 0.41 & 1.52 & 8.64 & 23 & 0.40 \\
\hline 940629 & 1410 & 2.12 & 0.113 & 8.9 & 40 & 47 & 0.45 & 1.95 & 8.03 & 22 & 0.51 \\
\hline 940629 & 2001 & 2.47 & 0.113 & 8.9 & 42 & 52 & 0.43 & 0.87 & 6.15 & 31 & 0.44 \\
\hline 940630 & 0159 & 2.06 & 0.113 & 8.9 & 42 & 50 & 0.40 & 1.74 & 9.21 & 22 & 0.47 \\
\hline 940630 & 0502 & 2.11 & 0.113 & 8.9 & 40 & 48 & 0.44 & 1.55 & 7.92 & 24 & 0.44 \\
\hline 940630 & 0803 & 2.02 & 0.113 & 8.9 & 40 & 48 & 0.44 & 9.69 & 8.34 & 24 & 0.44 \\
\hline 940630 & 1100 & 1.93 & 0.113 & 8.9 & 42 & 49 & 0.44 & 2.21 & 9.32 & 20 & 0.41 \\
\hline 940630 & 1400 & 2.09 & 0.113 & 8.9 & 44 & 47 & 0.39 & 2.08 & 11.78 & 16 & 0.32 \\
\hline 940630 & 1704 & 2.00 & 0.113 & 8.9 & 46 & 53 & 0.43 & 1.81 & 9.29 & 22 & 0.43 \\
\hline 940630 & 2001 & 2.15 & 0.113 & 8.9 & 46 & 52 & 0.42 & 1.76 & 9.64 & 21 & 0.50 \\
\hline 940701 & 0204 & 1.94 & 0.113 & 8.9 & 44 & 51 & 0.45 & 2.19 & 8.98 & 21 & 0.47 \\
\hline 940701 & 0804 & 1.81 & 0.113 & 8.9 & 50 & 57 & 0.46 & 2.66 & 9.87 & 19 & 0.63 \\
\hline 940701 & 1100 & 1.75 & 0.113 & 8.9 & 46 & 54 & 0.52 & 2.26 & 7.80 & 25 & 0.52 \\
\hline 940701 & 1403 & 1.72 & 0.123 & 8.2 & 42 & 49 & 0.50 & 2.14 & 8.03 & 24 & 0.44 \\
\hline 940701 & 2001 & 1.93 & 0.123 & 8.2 & 48 & 53 & 0.49 & 1.07 & 6.80 & 31 & 0.03 \\
\hline 940702 & 0202 & 1.91 & 0.132 & 7.6 & 42 & 50 & 0.49 & 1.32 & 6.77 & 32 & 0.13 \\
\hline 940702 & 1059 & 1.77 & 0.123 & 8.2 & 42 & 51 & 0.48 & 2.86 & 9.30 & 21 & 0.55 \\
\hline 940702 & 1403 & 1.88 & 0.123 & 8.2 & 42 & 49 & 0.48 & 2.79 & 9.34 & 19 & 0.46 \\
\hline 940702 & 2002 & 1.70 & 0.113 & 8.9 & 46 & 57 & 0.62 & 2.73 & 6.11 & 28 & 0.36 \\
\hline 940703 & 0202 & 1.61 & 0.123 & 8.2 & 42 & 56 & 0.74 & 3.28 & 4.77 & 34 & 0.58 \\
\hline 940703 & 0502 & 1.69 & 0.132 & 7.6 & 38 & 56 & 0.73 & 2.84 & 4.72 & 36 & 0.34 \\
\hline 940703 & 0802 & 1.66 & 0.142 & 7.0 & 42 & 57 & 0.77 & 3.01 & 4.24 & 37 & 0.58 \\
\hline 940703 & 1359 & 1.46 & 0.132 & 7.6 & 50 & 72 & 0.77 & 1.87 & 3.57 & 45 & 0.46 \\
\hline 940703 & 2002 & 1.69 & 0.113 & 8.9 & 48 & 68 & 0.74 & 2.23 & 3.95 & 40 & 0.47 \\
\hline 940704 & 0202 & 1.72 & 0.113 & 8.9 & 44 & 64 & 0.71 & 2.37 & 4.39 & 36 & 0.31 \\
\hline 940704 & 0502 & 1.77 & 0.103 & 9.7 & 44 & 66 & 0.74 & 2.01 & 3.80 & 41 & 0.29 \\
\hline 940704 & 0802 & 1.80 & 0.103 & 9.7 & 40 & 61 & 0.70 & 1.97 & 4.31 & 38 & 0.23 \\
\hline 940704 & 1101 & 1.90 & 0.113 & 8.9 & 42 & 62 & 0.67 & 1.97 & 4.50 & 38 & 0.32 \\
\hline 940704 & 1400 & 2.07 & 0.103 & 9.7 & 44 & 65 & 0.67 & 1.70 & 4.26 & 42 & 0.50 \\
\hline 940704 & 1703 & 2.03 & 0.103 & 9.7 & 46 & 68 & 0.68 & 1.64 & 4.16 & 42 & 0.27 \\
\hline 940704 & 2002 & 1.84 & 0.103 & 9.7 & 40 & 65 & 0.73 & 1.75 & 3.74 & 45 & 0.45 \\
\hline 940705 & 0202 & 1.65 & 0.113 & 8.9 & 40 & 60 & 0.73 & 2.53 & 4.22 & 40 & 0.71 \\
\hline 940705 & 0503 & 1.83 & 0.113 & 8.9 & 44 & 60 & 0.69 & 2.49 & 4.45 & 35 & 0.54 \\
\hline 940705 & 0802 & 1.69 & 0.103 & 9.7 & 42 & 61 & 0.76 & 2.58 & 3.80 & 41 & 1.25 \\
\hline 940705 & 1102 & 1.73 & 0.103 & 9.7 & 46 & 61 & 0.74 & 2.66 & 4.01 & 38 & 1.05 \\
\hline 940705 & 2005 & 1.62 & 0.103 & 9.7 & 46 & 61 & 0.75 & 2.82 & 4.13 & 35 & 0.89 \\
\hline 940706 & 0157 & 1.74 & 0.103 & 9.7 & 46 & 58 & 0.70 & 3.07 & 4.78 & 31 & 0.88 \\
\hline 940706 & 0834 & 1.59 & 0.093 & 10.7 & 46 & 59 & 0.69 & 2.82 & 4.72 & 31 & 0.72 \\
\hline 940706 & 1104 & 1.78 & 0.093 & 10.7 & 42 & 55 & 0.65 & 3.17 & 5.55 & 27 & 0.76 \\
\hline 940706 & 1402 & 1.63 & 0.103 & 9.7 & 46 & 59 & 0.66 & 2.62 & 4.94 & 30 & 0.75 \\
\hline 940706 & 1702 & 1.54 & 0.093 & 10.7 & 44 & 57 & 0.67 & 2.75 & 5.01 & 32 & 0.93 \\
\hline 940706 & 2003 & 1.43 & 0.103 & 9.7 & 46 & 62 & 0.69 & 2.54 & 4.63 & 33 & 0.69 \\
\hline 940707 & 0202 & 1.37 & 0.103 & 9.7 & 48 & 60 & 0.63 & 2.77 & 5.36 & 27 & 0.65 \\
\hline & 0502 & 1.27 & 0.103 & & 44 & 61 & 0.68 & 2.04 & 4.34 & 37 & 0.81 \\
\hline 940707 & 0802 & 1.24 & 0.093 & 10.7 & 46 & 66 & 0.71 & 1.85 & 3.77 & 42 & 0.77 \\
\hline 940707 & 1102 & 1.18 & 0.093 & 10.7 & 46 & 66 & 0.73 & 2.11 & 3.74 & 39 & 0.73 \\
\hline 940707 & 1402 & 1.18 & 0.093 & 10.7 & 46 & 64 & 0.74 & 2.27 & 3.89 & 39 & 0.96 \\
\hline 940707 & 1702 & 1.07 & 0.103 & 9.7 & 44 & 71 & 0.81 & 1.64 & 2.86 & 58 & 1.01 \\
\hline 940707 & 2002 & 1.07 & 0.103 & 9.7 & 58 & 74 & 0.82 & 1.58 & 2.88 & 58 & 0.98 \\
\hline 940708 & 0202 & 1.23 & 0.103 & 9.7 & 50 & 69 & 0.73 & 1.69 & 3.31 & 42 & 0.80 \\
\hline
\end{tabular}




\begin{tabular}{|c|c|c|c|c|c|c|c|c|c|c|c|}
\hline \multicolumn{12}{|c|}{ Table A1 (Continued) } \\
\hline Date & $\begin{array}{l}\text { Time } \\
\text { GMT }\end{array}$ & $\begin{array}{l}H_{m o} \\
\mathrm{~m}\end{array}$ & $\begin{array}{l}f_{p} \\
H z\end{array}$ & $\begin{array}{l}T_{p} \\
\text { sec }\end{array}$ & $\begin{array}{l}\theta_{p} \\
\operatorname{deg}\end{array}$ & $\begin{array}{l}\theta_{0} \\
\text { deg }\end{array}$ & 0 & $\mathrm{y}$ & $\delta$ & $\begin{array}{l}\Delta \theta \\
\operatorname{deg}\end{array}$ & A \\
\hline 940708 & 0459 & 1.24 & 0.103 & 9.7 & 50 & 67 & 0.76 & 1.61 & 3.25 & 47 & 0.68 \\
\hline 940708 & 0826 & 1.08 & 0.103 & 9.7 & 60 & 76 & 0.82 & 1.18 & 2.60 & 71 & 1.03 \\
\hline 940708 & 1102 & 1.18 & 0.103 & 9.7 & 48 & 78 & 0.87 & 1.14 & 2.25 & 91 & 1.44 \\
\hline 940708 & 1405 & 1.07 & 0.103 & 9.7 & 46 & 78 & 0.89 & 1.07 & 2.14 & 100 & 1.62 \\
\hline 940708 & 1702 & 1.07 & 0.064 & 15.6 & 44 & 81 & 0.89 & 1.05 & 2.11 & 101 & 1.25 \\
\hline 940708 & 2002 & 0.99 & 0.064 & 15.6 & 48 & 96 & 0.93 & 0.31 & 1.54 & 106 & 0.80 \\
\hline 940709 & 0202 & 0.88 & 0.064 & 15.6 & 44 & 101 & 0.93 & 0.14 & 1.69 & 101 & 0.24 \\
\hline 940709 & 0759 & 1.03 & 0.064 & 15.6 & 44 & 98 & 0.95 & 0.26 & 1.51 & 109 & 0.67 \\
\hline 940709 & 1102 & 0.96 & 0.064 & 15.6 & 46 & 98 & 0.95 & 0.31 & 1.63 & 104 & 0.65 \\
\hline 940709 & 1402 & 1.00 & 0.064 & 15.6 & 42 & 93 & 0.96 & 0.47 & 1.53 & 111 & 0.99 \\
\hline 940709 & 1702 & 1.14 & 0.103 & 9.7 & 38 & 77 & 0.97 & 1.15 & 1.89 & 116 & 1.70 \\
\hline 940709 & 2002 & 1.08 & 0.064 & 15.6 & 52 & 86 & 0.91 & 0.83 & 1.91 & 97 & 1.46 \\
\hline 940710 & 0202 & 0.93 & 0.064 & 15.6 & 154 & 102 & 0.96 & 0.20 & 1.54 & 106 & 0.40 \\
\hline 940710 & 1059 & 1.01 & 0.074 & 13.6 & 48 & 83 & 0.95 & 1.02 & 1.97 & 106 & 1.31 \\
\hline 940710 & 1359 & 1.04 & 0.074 & 13.6 & 40 & 82 & 0.97 & 1.01 & 1.90 & 110 & 1.28 \\
\hline 940711 & 0502 & 0.94 & 0.113 & 8.9 & 40 & 66 & 0.86 & 2.27 & 3.03 & 66 & 1.46 \\
\hline 940711 & 0802 & 0.98 & 0.113 & 8.9 & 40 & 64 & 0.89 & 2.35 & 2.94 & 74 & 1.64 \\
\hline 940711 & 1103 & 1.00 & 0.113 & 8.9 & 42 & 62 & 0.87 & 2.47 & 3.12 & 59 & 1.35 \\
\hline 940711 & 1703 & 1.06 & 0.113 & 8.9 & 40 & 66 & 0.84 & 2.26 & 3.19 & 50 & 0.58 \\
\hline 940711 & 2002 & 0.99 & 0.113 & 8.9 & 52 & 67 & 0.85 & 2.51 & 3.33 & 43 & 0.72 \\
\hline 940712 & 0202 & 0.96 & 0.113 & 8.9 & 48 & 68 & 0.86 & 2.07 & 3.00 & 51 & 0.76 \\
\hline 940712 & 0502 & 1.03 & 0.113 & 8.9 & 40 & 65 & 0.86 & 2.24 & 3.01 & 55 & 0.93 \\
\hline 940712 & 0806 & 1.12 & 0.113 & 8.9 & 38 & 60 & 0.76 & 2.57 & 3.85 & 40 & 0.64 \\
\hline 940712 & 1103 & 1.22 & 0.113 & 8.9 & 42 & 60 & 0.76 & 2.61 & 3.93 & 38 & 0.55 \\
\hline 940712 & 1402 & 1.27 & 0.113 & 8.9 & 42 & 60 & 0.79 & 2.60 & 3.77 & 39 & 0.83 \\
\hline 940712 & 2002 & 1.29 & 0.113 & 8.9 & 40 & 59 & 0.79 & 2.55 & 3.67 & 42 & 0.89 \\
\hline 940713 & 0503 & 1.32 & 0.113 & 8.9 & 42 & 62 & 0.78 & 2.36 & 3.58 & 41 & 0.72 \\
\hline 940713 & 0802 & 1.34 & 0.103 & 9.7 & 42 & 63 & 0.77 & 2.21 & 3.51 & 48 & 1.20 \\
\hline 940713 & 1102 & 1.41 & 0.103 & 9.7 & 42 & 61 & 0.74 & 2.23 & 3.73 & 44 & 1.14 \\
\hline 940713 & 1402 & 1.41 & 0.103 & 9.7 & 42 & 62 & 0.78 & 2.38 & 3.65 & 41 & 1.05 \\
\hline 940713 & 1702 & 1.57 & 0.103 & 9.7 & 44 & 59 & 0.66 & 2.35 & 4.74 & 37 & 0.80 \\
\hline 940713 & 2002 & 1.41 & 0.103 & 9.7 & 48 & 62 & 0.72 & 2.18 & 4.03 & 38 & 0.66 \\
\hline 940714 & 0200 & 1.26 & 0.103 & 9.7 & 48 & 66 & 0.73 & 2.13 & 3.74 & 37 & 0.79 \\
\hline 940714 & 0816 & 1.53 & 0.113 & 8.9 & 42 & 60 & 0.65 & 2.23 & 4.6 & 40 & 1.06 \\
\hline 940714 & 1101 & 1.40 & 0.123 & 8.2 & 44 & 66 & 0.72 & 1.89 & 3.78 & 45 & 0.50 \\
\hline 940714 & 1404 & 1.58 & 0.103 & 9.7 & 48 & 65 & 0.69 & 2.18 & 4.18 & 36 & 0.55 \\
\hline 940714 & 1702 & 1.70 & 0.103 & 9.7 & 42 & 61 & 0.68 & 2.31 & 4.47 & 39 & 1.16 \\
\hline 940714 & 2002 & 1.66 & 0.103 & 9.7 & 44 & 60 & 0.71 & 2.82 & 4.52 & 36 & 1.08 \\
\hline 940715 & 0202 & 1.50 & 0.103 & 9.7 & 48 & 66 & 0.74 & 2.4 & 3.8 & 37 & 1.08 \\
\hline 940715 & 0502 & 1.53 & 0.103 & 9.7 & 46 & 68 & 0.72 & 1.94 & 3.69 & 40 & 0.61 \\
\hline 940715 & 0802 & 1.44 & 0.103 & 9.7 & 42 & 67 & 0.77 & 2.14 & 3.48 & 45 & 0.86 \\
\hline 940715 & 1059 & 1.61 & 0.103 & 9.7 & 42 & 59 & 0.70 & 2.6 & 4. & 36 & 1.24 \\
\hline 940715 & 1758 & 2.03 & 0.093 & 10.7 & 42 & 53 & 0.62 & 3.13 & 5.73 & 23 & 0.92 \\
\hline 940715 & 1959 & 1.90 & 0.093 & 10.7 & 42 & 55 & 0.66 & 2.89 & 5.10 & 29 & 1.00 \\
\hline 940716 & 0202 & 1.80 & 0.093 & 10.7 & 42 & 56 & 0.67 & 3.19 & 4.99 & 24 & 1.09 \\
\hline 940716 & 0802 & 1.54 & 0.093 & 10. & 40 & 61 & 0.74 & 2.46 & 3.87 & 40 & 1.38 \\
\hline 940716 & 1105 & 1.54 & 0.103 & 9.7 & 40 & 61 & 0.76 & 2.33 & 3.75 & 41 & 1.17 \\
\hline 940716 & 1355 & 1.51 & 0.103 & 9.7 & 40 & 60 & 0.75 & 2.46 & 3.81 & 44 & 1.47 \\
\hline 940716 & 1659 & 1.34 & 0.103 & 9.7 & 42 & 70 & 0.83 & 1.65 & 2.78 & 70 & 1.44 \\
\hline 940716 & 2006 & 1.36 & 0.103 & 9.7 & 40 & 67 & 0.84 & 1.77 & 2.86 & 60 & 1.20 \\
\hline 940717 & 1359 & 1.18 & 0.093 & 10.7 & 40 & 71 & 0.87 & 1.43 & 2.41 & 90 & 1.79 \\
\hline
\end{tabular}




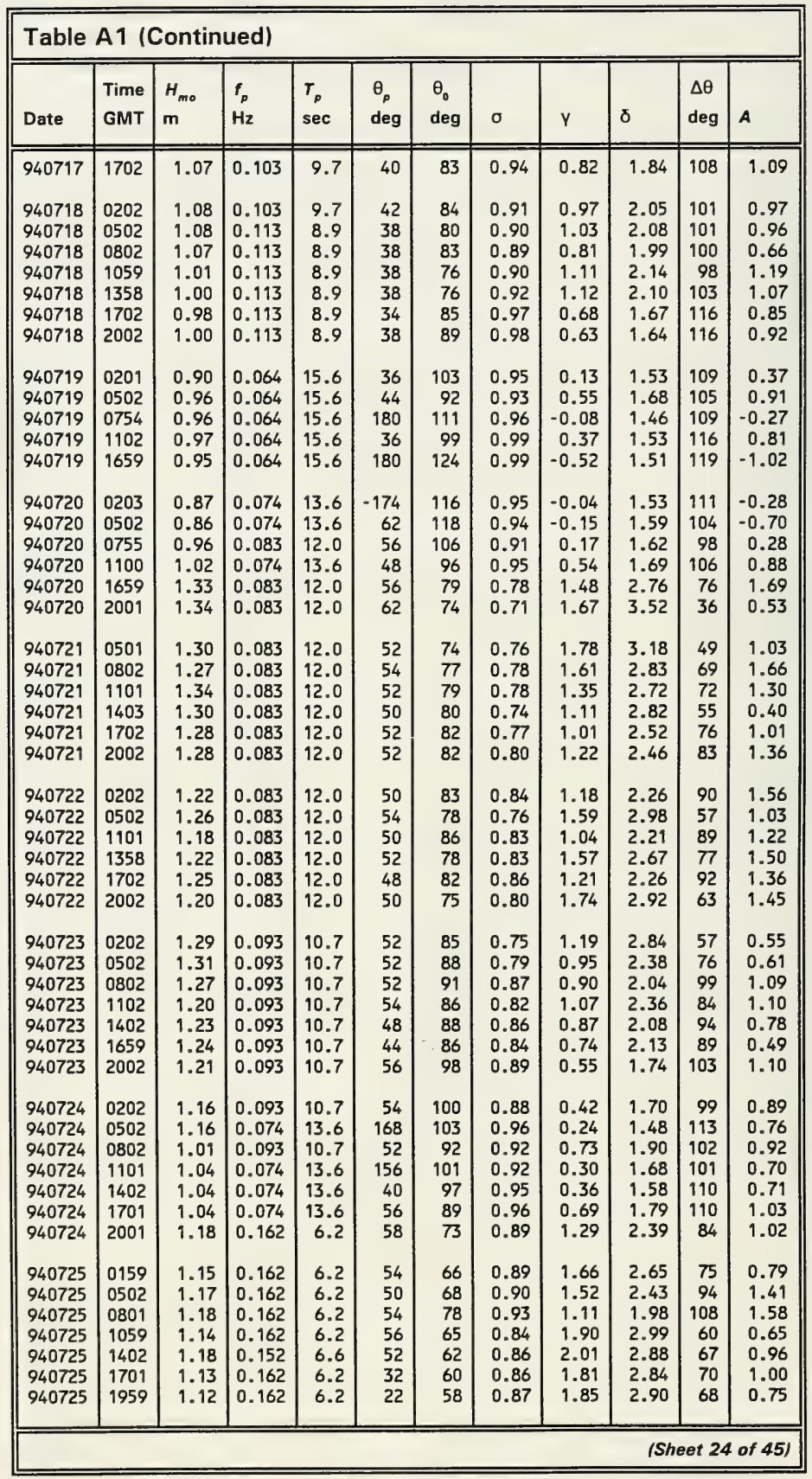




\begin{tabular}{|c|c|c|c|c|c|c|c|c|c|c|c|}
\hline Date & $\begin{array}{l}\text { Time } \\
\text { GMT }\end{array}$ & $\begin{array}{l}H_{m o} \\
m\end{array}$ & $\begin{array}{l}f_{p} \\
H_{z}\end{array}$ & $\begin{array}{l}T_{p} \\
\text { sec }\end{array}$ & $\begin{array}{l}\theta_{p} \\
\operatorname{deg}\end{array}$ & $\begin{array}{l}\theta_{0} \\
\text { deg }\end{array}$ & $\sigma$ & Y & $\delta$ & $\begin{array}{l}\Delta \theta \\
\operatorname{deg}\end{array}$ & A \\
\hline 940726 & 0202 & 0.90 & 0.162 & 6.2 & 50 & 72 & 0.89 & 1.36 & 2.40 & 88 & 1.30 \\
\hline 940726 & 0502 & 0.88 & 0.162 & 6.2 & 54 & 73 & 0.90 & 1.25 & 2.26 & 94 & 1.30 \\
\hline 940726 & 0827 & 0.86 & 0.162 & 6.2 & 44 & 81 & 0.95 & 1.01 & 1.94 & 107 & 1.35 \\
\hline 940726 & 1102 & 0.84 & 0.162 & 6.2 & 50 & 77 & 0.90 & 1.27 & 2.24 & 98 & 1.55 \\
\hline 940726 & 1402 & 1.07 & 0.162 & 6.2 & 28 & 61 & 0.81 & 1.90 & 3.15 & 59 & 0.83 \\
\hline 940726 & 1702 & 1.35 & 0.152 & 6.6 & 26 & 55 & 0.77 & 2.06 & 3.56 & 49 & 0.54 \\
\hline 940726 & 2002 & 1.70 & 0.142 & 7.0 & 26 & 43 & 0.62 & 2.39 & 5.65 & 30 & 0.15 \\
\hline 940727 & 0205 & 1.73 & 0.132 & 7.6 & 44 & 49 & 0.57 & 2.75 & 6.70 & 23 & 0.20 \\
\hline 940727 & 0505 & 1.81 & 0.123 & 8.2 & 42 & 47 & 0.58 & 2.92 & 6.40 & 22 & 0.19 \\
\hline 940727 & 0802 & 1.87 & 0.132 & 7.6 & 44 & 48 & 0.60 & 2.38 & 5.59 & 27 & 0.08 \\
\hline 940727 & 1101 & 1.86 & 0.132 & 7.6 & 44 & 46 & 0.59 & 2.55 & 6.20 & 28 & 0.10 \\
\hline 940727 & 1405 & 1.72 & 0.123 & 8.2 & 46 & 53 & 0.63 & 2.50 & 5.50 & 29 & 0.36 \\
\hline 940727 & 1659 & 1.88 & 0.132 & 7.6 & 38 & 54 & 0.64 & 1.64 & 4.41 & 44 & 0.68 \\
\hline 940727 & 1959 & 2.02 & 0.123 & 8.2 & 48 & 53 & 0.58 & 1.56 & 5.33 & 33 & 0.28 \\
\hline 940728 & 0201 & 1.69 & 0.123 & 8.2 & 48 & 50 & 0.59 & 2.22 & 6.13 & 29 & 0.10 \\
\hline 940728 & 0502 & 1.60 & 0.132 & 7.6 & 42 & 54 & 0.65 & 2.26 & 4.87 & 34 & 0.84 \\
\hline 940728 & 0802 & 1.65 & 0.123 & 8.2 & 36 & 55 & 0.62 & 1.93 & 5.09 & 37 & 0.50 \\
\hline 940728 & 1102 & 1.75 & 0.123 & 8.2 & 46 & 55 & 0.59 & 1.79 & 5.25 & 36 & 0.37 \\
\hline 940728 & 1402 & 1.70 & 0.123 & 8.2 & 44 & 55 & 0.58 & 2.30 & 5.85 & 28 & 0.59 \\
\hline 940728 & 1659 & 1.73 & 0.123 & 8.2 & 44 & 53 & 0.57 & 1.83 & 5.71 & 32 & 0.61 \\
\hline 940728 & 2001 & 1.88 & 0.123 & 8.2 & 46 & 50 & 0.57 & 1.60 & 5.49 & 33 & 0.15 \\
\hline 940729 & 0202 & 1.71 & 0.132 & 7.6 & 44 & 51 & 0.64 & 2.74 & 5.75 & 26 & 0.47 \\
\hline 940729 & 0459 & 1.77 & 0.123 & 8.2 & 40 & 49 & 0.60 & 2.53 & 5.90 & 26 & 0.51 \\
\hline 940729 & 0802 & 1.56 & 0.123 & 8.2 & 42 & 56 & 0.62 & 2.35 & 5.31 & 33 & 1.02 \\
\hline 940729 & 1058 & 1.55 & 0.132 & 7.6 & 44 & 63 & 0.65 & 1.52 & 4.13 & 50 & 1.21 \\
\hline 940729 & 1358 & 1.52 & 0.123 & 8.2 & 40 & 61 & 0.64 & 1.78 & 4.39 & 45 & 1.19 \\
\hline 940729 & 1651 & 1.38 & 0.123 & 8.2 & 42 & 64 & 0.69 & 1.93 & 4.09 & 45 & 0.95 \\
\hline 940729 & 2001 & 1.33 & 0.123 & 8.2 & 44 & 63 & 0.68 & 1.93 & 4.21 & 44 & 0.87 \\
\hline 940730 & 0202 & 1.29 & 0.123 & 8.2 & 48 & 67 & 0.72 & 2.06 & 3.95 & 44 & 1.05 \\
\hline 940730 & 0502 & 1.33 & 0.123 & 8.2 & 46 & 66 & 0.72 & 2.19 & 4.09 & 44 & 0.98 \\
\hline 940730 & 0802 & 1.33 & 0.132 & 7.6 & 44 & 67 & 0.71 & 1.82 & 3.92 & 47 & 0.91 \\
\hline 940730 & 1101 & 1.28 & 0.132 & 7.6 & 44 & 67 & 0.69 & 2.03 & 4.24 & 43 & 0.78 \\
\hline 940730 & 1401 & 1.13 & 0.142 & 7.0 & 46 & 72 & 0.73 & 1.77 & 3.74 & 47 & 0.69 \\
\hline 940730 & 1701 & 1.20 & 0.123 & 8.2 & 46 & 67 & 0.71 & 2.05 & 4.11 & 43 & 0.83 \\
\hline 940731 & 1059 & 1.42 & 0.113 & 8.9 & 42 & 64 & 0.71 & 1.76 & 3.55 & 47 & 1.02 \\
\hline 940731 & 1402 & 1.23 & 0.113 & 8.9 & 44 & 70 & 0.77 & 1.72 & 3.26 & 53 & 0.96 \\
\hline 940731 & 1701 & 1.44 & 0.113 & 8.9 & 40 & 67 & 0.79 & 2.02 & 3.30 & 50 & 0.92 \\
\hline 940731 & 1959 & 1.51 & 0.103 & 9.7 & 42 & 63 & 0.72 & 2.20 & 3.87 & 41 & 1.11 \\
\hline 940801 & 0159 & 1.40 & 0.074 & 13.6 & 44 & 69 & 0.74 & 1.86 & 3.30 & 46 & 0.96 \\
\hline 940801 & 0502 & 1.27 & 0.074 & 13.6 & 44 & 74 & 0.78 & 1.32 & 2.78 & 65 & 0.92 \\
\hline 940801 & 0801 & 1.39 & 0.074 & 13.6 & 44 & 69 & 0.73 & 1.57 & 3.33 & 48 & 0.69 \\
\hline 940801 & 1051 & 1.37 & 0.162 & 6.2 & 42 & 70 & 0.74 & 1.47 & 3.13 & 48 & 0.51 \\
\hline 940801 & 1357 & 1.33 & 0.162 & 6.2 & 42 & 67 & 0.76 & 1.91 & 3.38 & 46 & 0.70 \\
\hline 940801 & 1657 & 1.31 & 0.162 & 6.2 & 58 & 70 & 0.80 & 1.50 & 3.06 & 54 & 0.61 \\
\hline 940801 & 1958 & 1.40 & 0.162 & 6.2 & 56 & 70 & 0.75 & 1.58 & 3.20 & 44 & 0.60 \\
\hline 940802 & 0155 & 1.62 & 0.152 & 6.6 & 54 & 64 & 0.69 & 1.57 & 3.91 & 41 & 0.23 \\
\hline 940802 & 0458 & 1.86 & 0.132 & 7.6 & 38 & 57 & 0.65 & 1.91 & 4.38 & 40 & 0.56 \\
\hline 940802 & 0758 & 1.82 & 0.132 & 7.6 & 46 & 58 & 0.67 & 1.97 & 4.30 & 37 & 0.26 \\
\hline 940802 & 1112 & 1.78 & 0.132 & 7.6 & 44 & 58 & 0.64 & 2.02 & 4.63 & 36 & 0.29 \\
\hline 940802 & 1358 & 1.83 & 0.132 & 7.6 & 44 & 59 & 0.66 & 2.04 & 4.40 & 37 & 0.42 \\
\hline 940802 & 1658 & 1.74 & 0.132 & 7.6 & 40 & 57 & 0.72 & 2.08 & 3.87 & 42 & 0.61 \\
\hline 940802 & 1958 & 1.63 & 0.142 & 7.0 & 24 & 56 & 0.74 & 1.99 & 3.68 & 44 & 0.19 \\
\hline \multicolumn{12}{|c|}{ (Sheet 25 of 45) } \\
\hline
\end{tabular}




\begin{tabular}{|c|c|c|c|c|c|c|c|c|c|c|c|}
\hline Date & $\begin{array}{l}\text { Time } \\
\text { GMT }\end{array}$ & $\begin{array}{l}H_{m 0} \\
m\end{array}$ & $\begin{array}{l}f_{p} \\
H z\end{array}$ & $\begin{array}{l}T_{p} \\
\text { sec }\end{array}$ & $\begin{array}{l}\theta_{p} \\
\text { deg }\end{array}$ & $\begin{array}{l}\theta_{0} \\
\text { deg }\end{array}$ & $\sigma$ & $Y$ & $\delta$ & $\begin{array}{c}\Delta \theta \\
\text { deg }\end{array}$ & $A$ \\
\hline $\begin{array}{l}940803 \\
940803 \\
940803 \\
940803 \\
940803 \\
940803 \\
940803\end{array}$ & $\begin{array}{l}0155 \\
0458 \\
0758 \\
1058 \\
1355 \\
1655 \\
1959\end{array}$ & $\begin{array}{l}1.62 \\
1.68 \\
1.77 \\
2.00 \\
1.95 \\
1.81 \\
1.81\end{array}$ & $\begin{array}{l}0.142 \\
0.132 \\
0.132 \\
0.132 \\
0.132 \\
0.132 \\
0.132\end{array}$ & $\begin{array}{l}7.0 \\
7.6 \\
7.6 \\
7.6 \\
7.6 \\
7.6 \\
7.6\end{array}$ & $\begin{array}{l}26 \\
42 \\
34 \\
34 \\
34 \\
38 \\
62\end{array}$ & $\begin{array}{l}55 \\
56 \\
57 \\
57 \\
57 \\
58 \\
55\end{array}$ & $\begin{array}{l}0.71 \\
0.71 \\
0.71 \\
0.62 \\
0.62 \\
0.66 \\
0.63\end{array}$ & $\begin{array}{l}1.94 \\
2.13 \\
2.23 \\
1.63 \\
1.63 \\
1.95 \\
1.79\end{array}$ & $\begin{array}{l}3.80 \\
3.88 \\
3.95 \\
4.47 \\
4.50 \\
4.31 \\
4.76\end{array}$ & $\begin{array}{l}44 \\
39 \\
41 \\
43 \\
42 \\
37 \\
35\end{array}$ & $\begin{array}{r}0.15 \\
0.42 \\
0.46 \\
0.25 \\
0.33 \\
0.21 \\
-0.10\end{array}$ \\
\hline $\begin{array}{l}940804 \\
940804 \\
940804 \\
940804 \\
940804 \\
940804 \\
940804\end{array}$ & $\begin{array}{l}0200 \\
0500 \\
0800 \\
1059 \\
1358 \\
1659 \\
1959\end{array}$ & $\begin{array}{l}1.75 \\
1.83 \\
1.80 \\
1.87 \\
2.12 \\
2.10 \\
2.13\end{array}$ & $\begin{array}{l}0.132 \\
0.123 \\
0.132 \\
0.132 \\
0.132 \\
0.132 \\
0.123\end{array}$ & $\begin{array}{l}7.6 \\
8.2 \\
7.6 \\
7.6 \\
7.6 \\
7.6 \\
8.2\end{array}$ & $\begin{array}{l}48 \\
54 \\
44 \\
54 \\
48 \\
46 \\
42\end{array}$ & $\begin{array}{l}58 \\
57 \\
57 \\
56 \\
53 \\
52 \\
49\end{array}$ & $\begin{array}{l}0.62 \\
0.60 \\
0.65 \\
0.60 \\
0.58 \\
0.53 \\
0.53\end{array}$ & $\begin{array}{l}2.43 \\
2.13 \\
2.56 \\
2.36 \\
2.57 \\
2.18 \\
2.26\end{array}$ & $\begin{array}{l}5.07 \\
5.16 \\
4.81 \\
5.47 \\
5.89 \\
6.53 \\
6.88\end{array}$ & $\begin{array}{l}29 \\
31 \\
31 \\
27 \\
27 \\
26 \\
26\end{array}$ & $\begin{array}{l}0.24 \\
0.08 \\
0.48 \\
0.05 \\
0.11 \\
0.24 \\
0.06\end{array}$ \\
\hline $\begin{array}{l}940805 \\
940805 \\
940805 \\
940805 \\
940805 \\
940805 \\
940805\end{array}$ & $\begin{array}{l}0156 \\
0459 \\
0801 \\
1058 \\
1359 \\
1708 \\
1958\end{array}$ & $\begin{array}{l}1.88 \\
1.87 \\
1.79 \\
1.81 \\
1.74 \\
1.90 \\
1.94\end{array}$ & $\begin{array}{l}0.123 \\
0.123 \\
0.123 \\
0.123 \\
0.132 \\
0.123 \\
0.123\end{array}$ & $\begin{array}{l}8.2 \\
8.2 \\
8.2 \\
8.2 \\
7.6 \\
8.2 \\
8.2\end{array}$ & $\begin{array}{l}48 \\
44 \\
42 \\
46 \\
42 \\
42 \\
44\end{array}$ & $\begin{array}{l}53 \\
54 \\
54 \\
55 \\
53 \\
50 \\
50\end{array}$ & $\begin{array}{l}0.52 \\
0.53 \\
0.53 \\
0.55 \\
0.55 \\
0.51 \\
0.49\end{array}$ & $\begin{array}{l}2.41 \\
2.65 \\
2.85 \\
3.01 \\
2.84 \\
2.54 \\
2.15\end{array}$ & $\begin{array}{l}7.09 \\
7.18 \\
7.59 \\
7.07 \\
6.91 \\
7.67 \\
7.91\end{array}$ & $\begin{array}{l}25 \\
25 \\
25 \\
23 \\
24 \\
24 \\
26\end{array}$ & $\begin{array}{l}0.24 \\
0.52 \\
0.55 \\
0.37 \\
0.59 \\
0.33 \\
0.12\end{array}$ \\
\hline $\begin{array}{l}940806 \\
940806 \\
940806 \\
940806 \\
940806 \\
940806 \\
940806\end{array}$ & $\begin{array}{l}0200 \\
0500 \\
0759 \\
1059 \\
1357 \\
1659 \\
1959\end{array}$ & $\begin{array}{l}1.64 \\
1.56 \\
1.64 \\
1.59 \\
1.59 \\
1.61 \\
1.61\end{array}$ & $\begin{array}{l}0.123 \\
0.123 \\
0.113 \\
0.123 \\
0.113 \\
0.103 \\
0.103\end{array}$ & $\begin{array}{l}8.2 \\
8.2 \\
8.9 \\
8.2 \\
8.9 \\
9.7 \\
9.7\end{array}$ & $\begin{array}{l}48 \\
44 \\
42 \\
48 \\
44 \\
48 \\
48\end{array}$ & $\begin{array}{l}52 \\
55 \\
55 \\
57 \\
57 \\
58 \\
58\end{array}$ & $\begin{array}{l}0.58 \\
0.63 \\
0.60 \\
0.58 \\
0.64 \\
0.62 \\
0.65\end{array}$ & $\begin{array}{l}2.34 \\
2.90 \\
3.14 \\
3.08 \\
3.50 \\
3.31 \\
3.02\end{array}$ & $\begin{array}{l}6.20 \\
5.77 \\
6.39 \\
6.62 \\
6.17 \\
6.23 \\
5.74\end{array}$ & $\begin{array}{l}28 \\
31 \\
28 \\
26 \\
26 \\
25 \\
28\end{array}$ & $\begin{array}{l}0.15 \\
0.45 \\
0.46 \\
0.39 \\
0.84 \\
0.45 \\
0.35\end{array}$ \\
\hline $\begin{array}{l}940807 \\
940807 \\
940807 \\
940807 \\
940807 \\
940807 \\
940807\end{array}$ & $\begin{array}{l}0159 \\
0500 \\
0800 \\
1059 \\
1359 \\
1659 \\
1958\end{array}$ & $\begin{array}{l}1.72 \\
1.70 \\
1.60 \\
1.60 \\
1.61 \\
1.66 \\
1.55\end{array}$ & $\begin{array}{l}0.113 \\
0.113 \\
0.113 \\
0.113 \\
0.113 \\
0.113 \\
0.113\end{array}$ & $\begin{array}{l}8.9 \\
8.9 \\
8.9 \\
8.9 \\
8.9 \\
8.9 \\
8.9\end{array}$ & $\begin{array}{l}50 \\
54 \\
48 \\
50 \\
50 \\
40 \\
46\end{array}$ & $\begin{array}{l}63 \\
65 \\
63 \\
64 \\
62 \\
56 \\
60\end{array}$ & $\begin{array}{l}0.66 \\
0.65 \\
0.68 \\
0.65 \\
0.65 \\
0.63 \\
0.67\end{array}$ & $\begin{array}{l}2.69 \\
2.99 \\
3.01 \\
3.01 \\
3.17 \\
2.61 \\
2.84\end{array}$ & $\begin{array}{l}5.18 \\
5.35 \\
5.05 \\
5.51 \\
5.64 \\
5.50 \\
5.08\end{array}$ & $\begin{array}{l}34 \\
28 \\
31 \\
28 \\
28 \\
33 \\
32\end{array}$ & $\begin{array}{l}0.42 \\
0.41 \\
0.67 \\
0.66 \\
0.67 \\
0.70 \\
0.63\end{array}$ \\
\hline $\begin{array}{l}940808 \\
940808 \\
940808 \\
940808\end{array}$ & $\begin{array}{l}0456 \\
0755 \\
1101 \\
2005\end{array}$ & $\begin{array}{l}1.56 \\
1.61 \\
1.40 \\
1.78\end{array}$ & $\begin{array}{l}0.123 \\
0.123 \\
0.123 \\
0.113\end{array}$ & $\begin{array}{l}8.2 \\
8.2 \\
8.2 \\
8.9\end{array}$ & $\begin{array}{l}46 \\
46 \\
54 \\
40\end{array}$ & $\begin{array}{l}62 \\
61 \\
67 \\
57\end{array}$ & $\begin{array}{l}0.68 \\
0.70 \\
0.73 \\
0.62\end{array}$ & $\begin{array}{l}2.52 \\
2.98 \\
2.49 \\
2.38\end{array}$ & $\begin{array}{l}4.83 \\
4.81 \\
4.12 \\
5.48\end{array}$ & $\begin{array}{l}37 \\
33 \\
31 \\
36\end{array}$ & $\begin{array}{l}0.48 \\
0.41 \\
0.28 \\
0.64\end{array}$ \\
\hline $\begin{array}{l}940809 \\
940809 \\
940809 \\
940809 \\
940809 \\
940809\end{array}$ & $\begin{array}{l}0205 \\
0502 \\
1110 \\
1406 \\
1718 \\
2003\end{array}$ & $\begin{array}{l}1.72 \\
1.64 \\
1.60 \\
1.54 \\
1.44 \\
1.34\end{array}$ & $\begin{array}{l}0.113 \\
0.123 \\
0.123 \\
0.123 \\
0.132 \\
0.132\end{array}$ & $\begin{array}{l}8.9 \\
8.2 \\
8.2 \\
8.2 \\
7.6 \\
7.6\end{array}$ & $\begin{array}{l}54 \\
42 \\
64 \\
56 \\
56 \\
56\end{array}$ & $\begin{array}{l}65 \\
61 \\
62 \\
62 \\
61 \\
62\end{array}$ & $\begin{array}{l}0.67 \\
0.66 \\
0.68 \\
0.65 \\
0.63 \\
0.67\end{array}$ & $\begin{array}{l}2.16 \\
2.23 \\
1.97 \\
2.09 \\
2.18 \\
2.33\end{array}$ & $\begin{array}{l}4.62 \\
4.76 \\
4.64 \\
4.88 \\
5.02 \\
4.63\end{array}$ & $\begin{array}{l}36 \\
37 \\
36 \\
35 \\
34 \\
36\end{array}$ & $\begin{array}{r}0.27 \\
0.21 \\
-0.01 \\
0.15 \\
0.15 \\
0.24\end{array}$ \\
\hline $\begin{array}{l}940810 \\
940810 \\
940810 \\
940810 \\
940810 \\
940810\end{array}$ & $\begin{array}{l}0206 \\
0506 \\
0806 \\
1107 \\
1702 \\
2005\end{array}$ & $\begin{array}{l}1.13 \\
1.13 \\
1.15 \\
1.17 \\
1.07 \\
1.28\end{array}$ & $\begin{array}{l}0.142 \\
0.142 \\
0.152 \\
0.142 \\
0.152 \\
0.142\end{array}$ & $\begin{array}{l}7.0 \\
7.0 \\
6.6 \\
7.0 \\
6.6 \\
7.0\end{array}$ & $\begin{array}{l}72 \\
32 \\
28 \\
52 \\
54 \\
50\end{array}$ & $\begin{array}{l}70 \\
69 \\
61 \\
64 \\
73 \\
62\end{array}$ & $\begin{array}{l}0.79 \\
0.79 \\
0.76 \\
0.77 \\
0.84 \\
0.70\end{array}$ & $\begin{array}{l}1.50 \\
1.72 \\
1.76 \\
2.33 \\
2.12 \\
3.03\end{array}$ & $\begin{array}{l}3.15 \\
3.10 \\
3.50 \\
3.70 \\
3.08 \\
4.73\end{array}$ & $\begin{array}{l}48 \\
49 \\
52 \\
41 \\
52 \\
31\end{array}$ & $\begin{array}{r}-0.14 \\
0.22 \\
0.11 \\
0.34 \\
0.75 \\
0.53\end{array}$ \\
\hline 940811 & 0505 & 1.12 & 0.152 & 6.6 & 50 & 59 & 0.80 & 2.59 & 3.71 & 39 & 0.44 \\
\hline
\end{tabular}

(Sheet 26 of 45) 


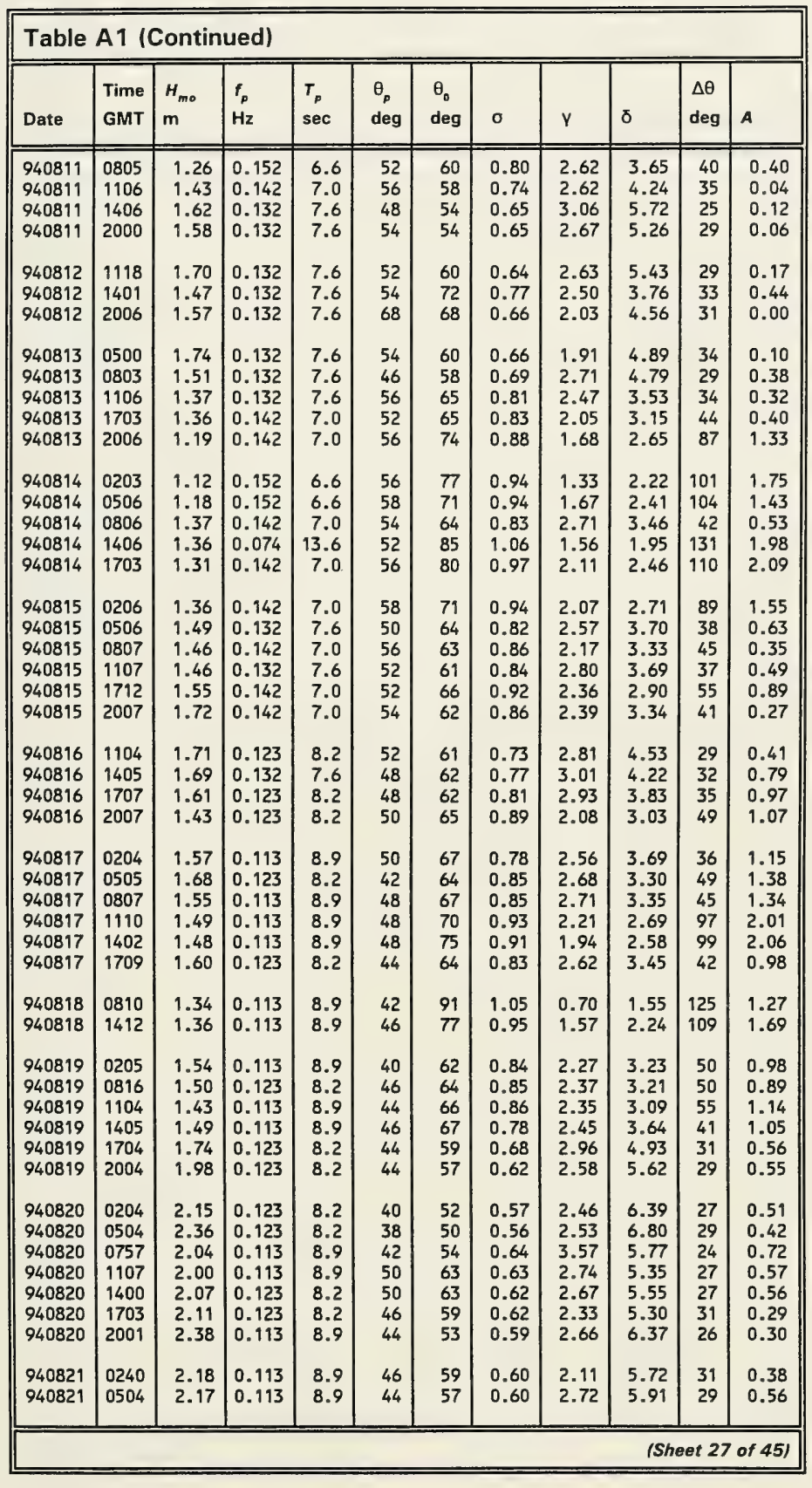




\begin{tabular}{|c|c|c|c|c|c|c|c|c|c|c|c|}
\hline Date & $\begin{array}{l}\text { Time } \\
\text { GMT }\end{array}$ & $\begin{array}{l}H_{m o} \\
\mathbf{m}\end{array}$ & $\begin{array}{l}f_{p} \\
\mathrm{~Hz}\end{array}$ & $\begin{array}{l}T_{p} \\
\text { sec }\end{array}$ & $\begin{array}{l}\theta_{p} \\
\text { deg }\end{array}$ & $\begin{array}{l}\theta_{0} \\
\operatorname{deg}\end{array}$ & 0 & $Y$ & $\delta$ & $\begin{array}{l}\Delta \theta \\
\operatorname{deg}\end{array}$ & $A$ \\
\hline 940821 & 0804 & 2.09 & 0.123 & 8.2 & 42 & 58 & 0.66 & 2.82 & 5.18 & 31 & 0.63 \\
\hline 940821 & 1401 & 1.69 & 0.123 & 8.2 & 52 & 72 & 0.71 & 2.31 & 4.22 & 33 & 0.41 \\
\hline 940821 & 1701 & 1.66 & 0.123 & 8.2 & 52 & 70 & 0.73 & 2.40 & 3.98 & 33 & 0.40 \\
\hline 940821 & 2004 & 1.89 & 0.123 & 8.2 & 64 & 66 & 0.66 & 2.55 & 4.80 & 28 & -0.02 \\
\hline 940822 & 0204 & 1.47 & 0.132 & 7.6 & 56 & 77 & 0.83 & 1.48 & 2.81 & 59 & 0.83 \\
\hline 940822 & 0501 & 1.70 & 0.132 & 7.6 & 52 & 66 & 0.79 & 2.08 & 3.55 & 41 & 0.43 \\
\hline 940822 & 0804 & 1.60 & 0.132 & 7.6 & 50 & 70 & 0.83 & 1.83 & 2.99 & 53 & 0.72 \\
\hline 940822 & 1101 & 1.48 & 0.132 & 7.6 & 50 & 73 & 0.81 & 1.92 & 3.27 & 46 & 0.48 \\
\hline 940822 & 1403 & 1.29 & 0.132 & 7.6 & 52 & 87 & 0.92 & 1.18 & 2.16 & 104 & 1.38 \\
\hline 940822 & 1703 & 1.26 & 0.132 & 7.6 & 52 & 90 & 0.97 & 0.85 & 1.81 & 112 & 1.27 \\
\hline 940822 & 1959 & 1.33 & 0.142 & 7.0 & 62 & 80 & 0.91 & 1.31 & 2.17 & 107 & 1.41 \\
\hline 940823 & 0802 & 1.14 & 0.054 & 18.5 & 62 & 96 & 1.01 & 0.52 & 1.60 & 115 & 1.24 \\
\hline 940823 & 1057 & 1.19 & 0.054 & 18.5 & 180 & 101 & 1.03 & 0.28 & 1.46 & 119 & 0.93 \\
\hline 940823 & 1655 & 1.27 & 0.132 & 7.6 & 56 & 91 & 0.96 & 0.84 & 1.79 & 110 & 1.21 \\
\hline 940823 & 2000 & 1.24 & 0.064 & 15.6 & 44 & 93 & 0.99 & 0.64 & 1.61 & 118 & 1.14 \\
\hline 940824 & 0201 & 1.15 & 0.162 & 6.2 & 56 & 82 & 0.94 & 1.12 & 2.12 & 106 & 1.43 \\
\hline 940824 & 0501 & 1.26 & 0.162 & 6.2 & 50 & 76 & 0.88 & 1.51 & 2.49 & 90 & 1.37 \\
\hline 940824 & 0801 & 1.17 & 0.162 & 6.2 & 56 & 82 & 0.92 & 1.17 & 2.10 & 107 & 1.35 \\
\hline 940824 & 1101 & 1.08 & 0.064 & 15.6 & 62 & 99 & 0.99 & 0.40 & 1.57 & 113 & 1.07 \\
\hline 940824 & 1406 & 1.21 & 0.064 & 15.6 & 48 & 85 & 0.95 & 0.95 & 1.90 & 113 & 1.26 \\
\hline 940824 & 1658 & 1.30 & 0.162 & 6.2 & 56 & 69 & 0.88 & 1.49 & 2.72 & 62 & 0.43 \\
\hline 940824 & 2000 & 1.47 & 0.162 & 6.2 & 26 & 61 & 0.81 & 1.85 & 3.21 & 52 & 0.26 \\
\hline 940825 & 0200 & 1.54 & 0.152 & 6.6 & 66 & 67 & 0.72 & 1.99 & 3.98 & 36 & 0.07 \\
\hline 940825 & 0500 & 1.45 & 0.142 & 7.0 & 50 & 67 & 0.74 & 2.09 & 3.80 & 37 & 0.36 \\
\hline 940825 & 0800 & 1.45 & 0.132 & 7.6 & 58 & 64 & 0.75 & 1.86 & 3.66 & 40 & 0.17 \\
\hline 940825 & 1058 & 1.40 & 0.142 & 7.0 & 40 & 61 & 0.79 & 2.12 & 3.52 & 44 & 0.39 \\
\hline 940825 & 1702 & 1.35 & 0.113 & 8.9 & 26 & 65 & 0.82 & 1.59 & 2.88 & 58 & 0.16 \\
\hline 940825 & 2001 & 1.28 & 0.113 & 8.9 & 36 & 63 & 0.83 & 1.79 & 3.05 & 55 & 0.48 \\
\hline 940826 & 0201 & 1.17 & 0.064 & 15.6 & 36 & 82 & 0.91 & 0.87 & 1.99 & 104 & 0.94 \\
\hline 940826 & 0501 & 1.18 & 0.162 & 6.2 & 68 & 73 & 0.81 & 1.54 & 2.93 & 55 & 0.44 \\
\hline 940826 & 0803 & 1.29 & 0.152 & 6.6 & 32 & 66 & 0.80 & 1.67 & 3.07 & 53 & 0.35 \\
\hline 940826 & 1102 & 1.29 & 0.162 & 6.2 & 34 & 65 & 0.85 & 1.80 & 2.82 & 63 & 0.83 \\
\hline 940826 & 1410 & 1.17 & 0.162 & 6.2 & 56 & 77 & 0.93 & 1.15 & 2.11 & 106 & 1.30 \\
\hline 940826 & 1700 & 1.25 & 0.162 & 6.2 & 50 & 76 & 0.88 & 1.31 & 2.36 & 92 & 1.33 \\
\hline 940826 & 2000 & 1.14 & 0.162 & 6.2 & 34 & 82 & 0.95 & 1.05 & 1.93 & 111 & 1.32 \\
\hline 940827 & 0203 & 0.94 & 0.064 & 15.6 & 162 & 117 & 0.96 & -0.56 & 1.58 & 112 & -1.24 \\
\hline & 0503 & 0.88 & 0.064 & 15.6 & 150 & 121 & 0.94 & -0.52 & 1.75 & 104 & -1.18 \\
\hline 940827 & 0803 & 0.86 & 0.064 & 15.6 & -176 & 116 & 0.98 & -0.26 & 1.56 & 112 & -0.59 \\
\hline 940827 & 1101 & 0.89 & 0.064 & 15.6 & 154 & 129 & 0.92 & -0.74 & 1.92 & 100 & -1.17 \\
\hline 940827 & 1402 & 0.78 & 0.064 & 15.6 & -180 & 125 & 0.92 & -0.49 & 1.84 & 104 & -0.73 \\
\hline 940827 & 1702 & 0.78 & 0.064 & 15.6 & 152 & 126 & 0.92 & -0.54 & 1.84 & 104 & -1.01 \\
\hline 940827 & 2005 & 0.91 & 0.064 & 15.6 & -178 & 109 & 0.97 & 0.08 & 1.52 & 111 & 0.08 \\
\hline 940827 & 2301 & 0.87 & 0.064 & 15.6 & 150 & 110 & 0.92 & -0.02 & 1.61 & 99 & -0.18 \\
\hline 940828 & 0159 & 0.89 & 0.064 & 15.6 & 154 & 114 & 0.91 & -0.20 & 1.61 & 100 & -0.55 \\
\hline 940828 & 0458 & 0.87 & 0.064 & 15.6 & 150 & 114 & 0.89 & -0.16 & 1.73 & 94 & -0.43 \\
\hline 940828 & 0801 & 0.86 & 0.064 & 15.6 & 152 & 105 & 0.90 & 0.10 & 1.76 & 92 & 0.56 \\
\hline 940828 & 1401 & 1.03 & 0.054 & 18.5 & 150 & 105 & 0.95 & -0.11 & 1.55 & 104 & -0.32 \\
\hline 940829 & 0201 & 1.71 & 0.152 & 6.6 & 26 & 60 & 0.75 & 1.53 & 3.21 & 53 & 0.39 \\
\hline 940829 & 0501 & 1.86 & 0.142 & 7.0 & 28 & 62 & 0.75 & 1.57 & 3.10 & 52 & 0.35 \\
\hline 940829 & 0801 & 1.84 & 0.142 & 7.0 & 46 & 57 & 0.73 & 1.92 & 3.61 & 47 & 0.63 \\
\hline 940829 & 1101 & 1.89 & 0.132 & 7.6 & 48 & 59 & 0.73 & 1.80 & 3.38 & 48 & 0.66 \\
\hline 940829 & 1401 & 1.79 & 0.142 & 7.0 & 30 & 56 & 0.73 & 2.09 & 3.76 & 44 & 0.62 \\
\hline \multicolumn{12}{|c|}{ (Sheet 28 of 45 ) } \\
\hline
\end{tabular}




\begin{tabular}{|c|c|c|c|c|c|c|c|c|c|c|c|}
\hline Date & $\begin{array}{l}\text { Time } \\
\text { GMT }\end{array}$ & $\begin{array}{l}H_{m 0} \\
\mathrm{~m}\end{array}$ & $\begin{array}{l}f_{b} \\
\mathrm{~Hz}\end{array}$ & $\begin{array}{l}T_{p} \\
\text { sec }\end{array}$ & $\begin{array}{l}\theta_{p} \\
\text { deg }\end{array}$ & $\begin{array}{l}\theta_{0} \\
\operatorname{deg}\end{array}$ & 0 & $\mathrm{Y}$ & $\delta$ & $\begin{array}{l}\Delta \theta \\
\operatorname{deg}\end{array}$ & A \\
\hline 940829 & 2001 & 1.75 & 0.142 & 7.0 & 48 & 65 & 0.74 & 1.44 & 3.21 & 49 & 0.59 \\
\hline 940830 & 0201 & 1.54 & 0.132 & 7.6 & 62 & 76 & 0.73 & 1.17 & 2.79 & 54 & 0.54 \\
\hline 940830 & 0458 & 1.89 & 0.123 & 8.2 & 56 & 68 & 0.68 & 1.65 & 3.59 & 42 & 0.45 \\
\hline 940830 & 0801 & 2.26 & 0.123 & 8.2 & 38 & 60 & 0.57 & 1.60 & 5.05 & 36 & 0.19 \\
\hline 940830 & 1101 & 2.30 & 0.123 & 8.2 & 56 & 61 & 0.58 & 1.71 & 5.04 & 34 & 0.11 \\
\hline 940830 & 1401 & 2.05 & 0.123 & 8.2 & 62 & 66 & 0.63 & 1.69 & 4.36 & 33 & -0.01 \\
\hline 940830 & 1708 & 1.71 & 0.123 & 8.2 & 68 & 67 & 0.69 & 1.59 & 3.78 & 38 & -0.03 \\
\hline 940830 & 2001 & 1.73 & 0.132 & 7.6 & 68 & 66 & 0.61 & 1.73 & 4.65 & 31 & -0.27 \\
\hline 940831 & 0201 & 1.59 & 0.123 & 8.2 & 56 & 68 & 0.63 & 2.12 & 4.66 & 29 & 0.27 \\
\hline 940831 & 0459 & 1.65 & 0.123 & 8.2 & 46 & 62 & 0.62 & 2.45 & 5.00 & 29 & 0.54 \\
\hline 940831 & 0800 & 1.65 & 0.123 & 8.2 & 46 & 61 & 0.62 & 2.47 & 4.92 & 28 & 0.61 \\
\hline 940831 & 1059 & 1.57 & 0.113 & 8.9 & 46 & 62 & 0.65 & 2.61 & 4.69 & 29 & 0.93 \\
\hline 940831 & 1958 & 1.68 & 0.113 & 8.9 & 42 & 58 & 0.63 & 2.63 & 5.22 & 28 & 0.36 \\
\hline 940901 & 0158 & 1.31 & 0.113 & 8.9 & 44 & 62 & 0.69 & 2.39 & 4.32 & 36 & 0.80 \\
\hline 940901 & 0457 & 1.30 & 0.123 & 8.2 & 46 & 68 & 0.77 & 1.82 & 3.14 & 50 & 0.96 \\
\hline 940901 & 0752 & 1.37 & 0.142 & 7.0 & 44 & 61 & 0.71 & 2.71 & 4.25 & 35 & 1.12 \\
\hline 940901 & 1059 & 1.40 & 0.123 & 8.2 & 42 & 61 & 0.76 & 2.42 & 3.71 & 41 & 0.92 \\
\hline 940901 & 1358 & 1.30 & 0.132 & 7.6 & 44 & 64 & 0.75 & 2.07 & 3.64 & 47 & 1.37 \\
\hline 940901 & 1651 & 1.32 & 0.064 & 15.6 & 46 & 67 & 0.78 & 2.06 & 3.22 & 49 & 1.18 \\
\hline 940901 & 1956 & 1.25 & 0.064 & 15.6 & 46 & 67 & 0.80 & 2.04 & 3.31 & 46 & 0.83 \\
\hline 940902 & 0156 & 1.21 & 0.113 & 8.9 & 44 & 65 & 0.79 & 2.16 & 3.25 & 51 & 1.42 \\
\hline 940902 & 0459 & 1.51 & 0.113 & 8.9 & 38 & 58 & 0.66 & 2.38 & 4.64 & 37 & 0.74 \\
\hline 940902 & 0759 & 1.65 & 0.123 & 8.2 & 44 & 58 & 0.63 & 3.15 & 5.92 & 27 & 0.29 \\
\hline 940902 & 1358 & 1.55 & 0.113 & 8.9 & 50 & 63 & 0.64 & 2.58 & 5.06 & 29 & 0.68 \\
\hline 940902 & 1703 & 1.72 & 0.123 & 8.2 & 46 & 59 & 0.60 & 2.62 & 5.69 & 30 & 0.73 \\
\hline 940903 & 0153 & 2.10 & 0.123 & 8.2 & 40 & 56 & 0.50 & 2.21 & 7.24 & 30 & 0.33 \\
\hline 940903 & 0456 & 1.77 & 0.123 & 8.2 & 42 & 59 & 0.59 & 2.34 & 5.74 & 34 & 0.55 \\
\hline 940903 & 0759 & 1.72 & 0.123 & 8.2 & 48 & 62 & 0.57 & 1.99 & 5.85 & 31 & 0.33 \\
\hline 940903 & 1059 & 1.66 & 0.132 & 7.6 & 44 & 59 & 0.60 & 2.12 & 5.49 & 33 & 0.52 \\
\hline 940903 & 1359 & 1.58 & 0.132 & 7.6 & 48 & 61 & 0.62 & 1.98 & 5.28 & 34 & 0.42 \\
\hline 940903 & 1653 & 1.59 & 0.142 & 7.0 & 46 & 57 & 0.65 & 2.04 & 5.10 & 36 & 0.49 \\
\hline 940903 & 1959 & 1.60 & 0.142 & 7.0 & 48 & 59 & 0.66 & 2.32 & 5.14 & 30 & 0.02 \\
\hline 940904 & 0151 & 1.44 & 0.132 & 7.6 & 52 & 66 & 0.68 & 2.40 & 4.78 & 29 & 0.17 \\
\hline 940904 & 0759 & 1.50 & 0.132 & 7.6 & 56 & 72 & 0.66 & 2.56 & 4.80 & 27 & 0.26 \\
\hline 940904 & 1359 & 1.55 & 0.132 & 7.6 & 54 & 69 & 0.65 & 2.41 & 4.82 & 27 & 0.16 \\
\hline 940904 & 1659 & 1.58 & 0.132 & 7.6 & 56 & 68 & 0.62 & 2.84 & 5.52 & 24 & 0.45 \\
\hline 940905 & 0157 & 1.43 & 0.093 & 10.7 & 54 & 71 & 0.73 & 2.19 & 3.89 & 32 & 0.33 \\
\hline 940905 & 0459 & 1.50 & 0.103 & 9.7 & 54 & 69 & 0.73 & 2.25 & 4.01 & 34 & 0.41 \\
\hline 940905 & 0759 & 1.50 & 0.093 & 10.7 & 64 & 71 & 0.72 & 2.23 & 3.96 & 31 & 0.28 \\
\hline 940905 & 1100 & 1.52 & 0.093 & 10.7 & 54 & 72 & 0.74 & 2.15 & 3.74 & 34 & 0.44 \\
\hline 940905 & 1659 & 1.75 & 0.093 & 10.7 & 54 & 66 & 0.61 & 2.62 & 5.44 & 26 & 0.44 \\
\hline 940905 & 1959 & 1.56 & 0.093 & 10.7 & 52 & 69 & 0.70 & 2.04 & 4.01 & 34 & 0.34 \\
\hline 940906 & 0459 & 1.57 & 0.103 & 9.7 & 66 & 70 & 0.72 & 1.74 & 3.66 & 38 & 0.02 \\
\hline 940906 & 0759 & 1.56 & 0.103 & 9.7 & 64 & 72 & 0.76 & 1.68 & 3.22 & 43 & 0.29 \\
\hline 940906 & 1547 & 1.34 & 0.113 & 8.9 & 34 & 70 & 0.80 & 1.66 & 3.03 & 52 & 0.34 \\
\hline 940906 & 1730 & 1.30 & 0.103 & 9.7 & 64 & 78 & 0.83 & 1.31 & 2.67 & 63 & 0.59 \\
\hline 940906 & 1959 & 1.22 & 0.103 & 9.7 & 68 & 86 & 0.91 & 0.97 & 2.05 & 100 & 1.11 \\
\hline & 0151 & 1.14 & 0.113 & 8.9 & 70 & 91 & 0.93 & 0.82 & 1.87 & 100 & 1.35 \\
\hline 940907 & 0459 & 1.06 & 0.054 & 18.5 & 70 & 105 & 0.92 & 0.41 & 1.64 & 100 & 1.30 \\
\hline 940907 & 0800 & 1.08 & 0.064 & 15.6 & 68 & 105 & 0.93 & 0.42 & 1.59 & 103 & 1.27 \\
\hline 940907 & 1356 & 1.07 & 0.074 & 13.6 & 54 & 107 & 0.99 & 0.30 & 1.51 & 112 & 0.78 \\
\hline
\end{tabular}




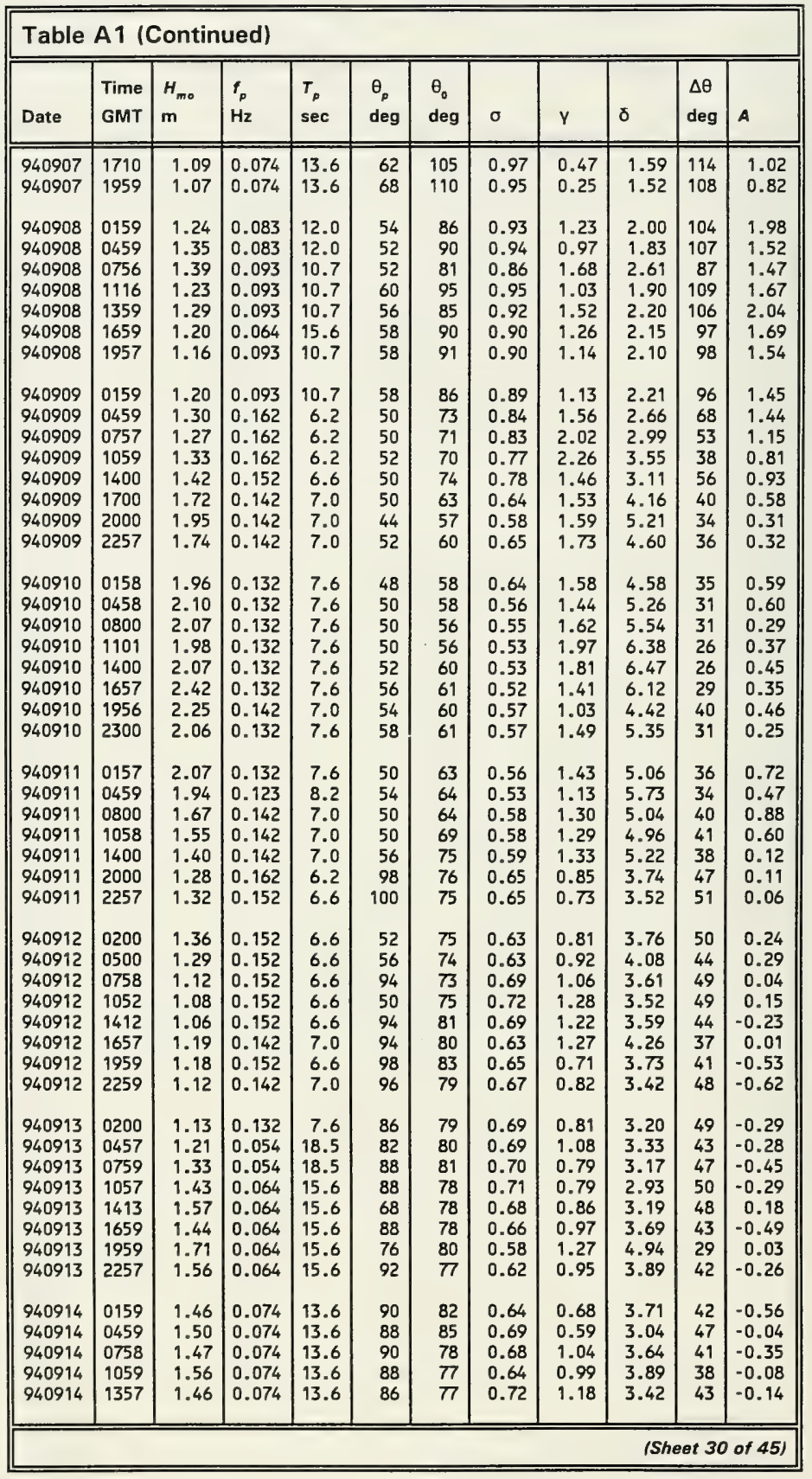




\begin{tabular}{|c|c|c|c|c|c|c|c|c|c|c|c|}
\hline Date & $\begin{array}{l}\text { Time } \\
\text { GMT }\end{array}$ & $\begin{array}{l}H_{m 0} \\
m\end{array}$ & $\begin{array}{l}f_{p} \\
\mathrm{~Hz}\end{array}$ & $\begin{array}{l}T_{p} \\
\text { sec }\end{array}$ & $\begin{array}{l}\theta_{p} \\
\text { deg }\end{array}$ & $\begin{array}{l}\theta_{0} \\
\text { deg }\end{array}$ & 0 & $\mathrm{Y}$ & $\delta$ & $\begin{array}{l}\Delta \theta \\
\operatorname{deg}\end{array}$ & A \\
\hline 940914 & 1701 & 1.44 & 0.074 & 13.6 & 88 & 81 & 0.73 & 0.97 & 3.22 & 44 & -0.26 \\
\hline 940914 & 2000 & 1.44 & 0.074 & 13.6 & 88 & 86 & 0.73 & 1.09 & 3.21 & 46 & 0.02 \\
\hline 940914 & 2300 & 1.28 & 0.083 & 12.0 & 58 & 78 & 0.73 & 1.47 & 3.48 & 43 & 0.35 \\
\hline 940915 & 0200 & 1.20 & 0.083 & 12.0 & 84 & 85 & 0.76 & 1.25 & 3.04 & 49 & 0.13 \\
\hline 940915 & 0757 & 1.26 & 0.083 & 12.0 & 86 & 82 & 0.72 & 1.26 & 3.31 & 41 & -0.18 \\
\hline 940915 & 1114 & 1.25 & 0.083 & 12.0 & 86 & 78 & 0.67 & 1.30 & 3.88 & 39 & -0.22 \\
\hline 940915 & 1402 & 1.15 & 0.162 & 6.2 & 56 & 78 & 0.74 & 1.60 & 3.43 & 43 & 0.17 \\
\hline 940915 & 1657 & 1.04 & 0.093 & 10.7 & 84 & 87 & 0.80 & 1.38 & 2.83 & 60 & 0.65 \\
\hline 940915 & 1951 & 0.94 & 0.074 & 13.6 & 84 & 94 & 0.84 & 0.92 & 2.41 & 82 & 0.95 \\
\hline 940915 & 2300 & 0.89 & 0.093 & 10.7 & 84 & 94 & 0.80 & 0.82 & 2.50 & 76 & 0.96 \\
\hline 940916 & 0157 & 0.99 & 0.074 & 13.6 & 60 & 87 & 0.82 & 1.24 & 2.58 & 76 & 1.14 \\
\hline 940916 & 0500 & 0.99 & 0.083 & 12.0 & 56 & 85 & 0.83 & 1.06 & 2.55 & 75 & 1.11 \\
\hline 940916 & 0757 & 1.04 & 0.083 & 12.0 & 52 & 80 & 0.81 & 1.25 & 2.78 & 66 & 0.76 \\
\hline 940916 & 1059 & 1.11 & 0.083 & 12.0 & 46 & 69 & 0.81 & 1.83 & 3.15 & 53 & 0.83 \\
\hline 940916 & 1400 & 1.05 & 0.103 & 9.7 & 48 & 77 & 0.80 & 1.53 & 2.95 & 54 & 0.47 \\
\hline 940916 & 1659 & 1.12 & 0.103 & 9.7 & 48 & 74 & 0.79 & 1.52 & 3.14 & 50 & 0.26 \\
\hline 940916 & 1959 & 1.11 & 0.093 & 10.7 & 54 & 76 & 0.74 & 1.57 & 3.36 & 43 & 0.35 \\
\hline 940916 & 2259 & 1.09 & 0.103 & 9.7 & 52 & 71 & 0.75 & 1.72 & 3.55 & 43 & 0.45 \\
\hline 940917 & 0159 & 1.05 & 0.113 & 8.9 & 48 & 72 & 0.79 & 1.83 & 3.22 & 49 & 0.75 \\
\hline 940917 & 0500 & 1.05 & 0.132 & 7.6 & 48 & 69 & 0.75 & 1.97 & 3.40 & 45 & 0.82 \\
\hline 940917 & 0800 & 1.04 & 0.103 & 9.7 & 48 & 71 & 0.78 & 1.60 & 3.07 & 52 & 1.01 \\
\hline 940917 & 1057 & 1.04 & 0.113 & 8.9 & 40 & 66 & 0.77 & 1.53 & 3.18 & 54 & 1.11 \\
\hline 940917 & 1400 & 1.01 & 0.103 & 9.7 & 38 & 74 & 0.83 & 1.04 & 2.46 & 74 & 0.66 \\
\hline 940917 & 1659 & 1.04 & 0.074 & 13.6 & 48 & 73 & 0.81 & 1.48 & 2.79 & 66 & 1.20 \\
\hline 940917 & 1959 & 1.11 & 0.074 & 13.6 & 48 & 74 & 0.80 & 1.48 & 2.82 & 69 & 1.54 \\
\hline 940917 & 2259 & 1.31 & 0.074 & 13.6 & 44 & 65 & 0.77 & 1.99 & 3.55 & 46 & 1.42 \\
\hline 940918 & 0157 & 1.41 & 0.074 & 13.6 & 46 & 64 & 0.69 & 1.84 & 3.88 & 41 & 1.26 \\
\hline 940918 & 0500 & 1.49 & 0.074 & 13.6 & 50 & 63 & 0.64 & 2.51 & 4.84 & 28 & 1.26 \\
\hline 940918 & 0759 & 1.52 & 0.074 & 13.6 & 48 & 62 & 0.64 & 2.30 & 4.81 & 32 & 1.30 \\
\hline 940918 & 1059 & 1.36 & 0.074 & 13.6 & 48 & 61 & 0.65 & 2.13 & 4.74 & 32 & 1.22 \\
\hline 940918 & 1359 & 1.38 & 0.074 & 13.6 & 46 & 67 & 0.67 & 1.23 & 3.58 & 52 & 1.08 \\
\hline 940918 & 1657 & 1.33 & 0.074 & 13.6 & 52 & 71 & 0.70 & 1.07 & 3.08 & 53 & 1.10 \\
\hline 940918 & 1959 & 1.33 & 0.074 & 13.6 & 50 & 72 & 0.66 & 1.38 & 3.75 & 44 & 0.58 \\
\hline 940919 & 0459 & 1.35 & 0.083 & 12.0 & 52 & 74 & 0.72 & 1.34 & 3.48 & 46 & 0.37 \\
\hline 940919 & 0757 & 1.32 & 0.074 & 13.6 & 50 & 70 & 0.64 & 1.61 & 4.21 & 40 & 0.71 \\
\hline 940919 & 1134 & 1.34 & 0.083 & 12.0 & 48 & 67 & 0.62 & 1.50 & 4.56 & 41 & 0.83 \\
\hline 940919 & 1400 & 1.32 & 0.083 & 12.0 & 44 & 68 & 0.70 & 1.08 & 3.29 & 53 & 0.79 \\
\hline 940919 & 1659 & 1.47 & 0.083 & 12.0 & 90 & 67 & 0.64 & 0.77 & 3.66 & 48 & 0.04 \\
\hline 940919 & 2257 & 1.56 & 0.083 & 12.0 & 48 & 63 & 0.58 & 1.43 & 5.13 & 38 & 0.64 \\
\hline 940920 & 0200 & 1.53 & 0.103 & 9.7 & 42 & 64 & 0.62 & 1.08 & 4.07 & 48 & 0.66 \\
\hline 940920 & 0458 & 1.54 & 0.103 & 9.7 & 44 & 60 & 0.59 & 1.62 & 4.85 & 42 & 0.91 \\
\hline 940920 & 0800 & 1.53 & 0.103 & 9.7 & 42 & 58 & 0.60 & 2.03 & 5.46 & 36 & 0.67 \\
\hline 940920 & 1659 & 1.43 & 0.103 & 9.7 & 44 & 59 & 0.64 & 2.11 & 5.15 & 38 & 0.94 \\
\hline 940920 & 2259 & 1.48 & 0.093 & 10.7 & 52 & 65 & 0.63 & 2.49 & 5.32 & 30 & 0.46 \\
\hline 940921 & 0159 & 1.52 & 0.103 & 9.7 & 42 & 59 & 0.61 & 1.98 & 5.44 & 37 & 0.58 \\
\hline 940921 & 0847 & 1.52 & 0.093 & 10.7 & 48 & 62 & 0.62 & 2.3 & 5.52 & 34 & 0.64 \\
\hline 940921 & 1115 & 1.53 & 0.093 & 10.7 & 46 & 61 & 0.65 & 2.2 & 5.08 & 37 & 0.92 \\
\hline 940921 & 1400 & 1.48 & 0.093 & 10.7 & 44 & 60 & 0.64 & 1.98 & 4.96 & 40 & 0.89 \\
\hline 940921 & 1646 & 1.39 & 0.093 & 10.7 & 50 & 63 & 0.66 & 2.50 & 5.05 & 34 & 0.90 \\
\hline 940921 & 2000 & 1.24 & 0.093 & 10.7 & 46 & 62 & 0.74 & 2.31 & 4.08 & 42 & 0.98 \\
\hline 940921 & 2300 & 1.38 & 0.093 & 10.7 & 42 & 64 & 0.74 & 1.79 & 3.63 & 51 & 1.04 \\
\hline 940922 & 0200 & 1.33 & 0.093 & 10.7 & 42 & 66 & 0.75 & 1.77 & 3.48 & 50 & 1.19 \\
\hline \multicolumn{12}{|c|}{ (Sheet 31 of 45) } \\
\hline
\end{tabular}




\begin{tabular}{|c|c|c|c|c|c|c|c|c|c|c|c|}
\hline \multicolumn{12}{|c|}{ Table A1 (Continued) } \\
\hline Date & $\begin{array}{l}\text { Time } \\
\text { GMT }\end{array}$ & $\begin{array}{l}H_{m o} \\
m\end{array}$ & $\begin{array}{l}f_{p} \\
\mathrm{~Hz}\end{array}$ & $\begin{array}{l}T_{p} \\
\text { sec }\end{array}$ & $\begin{array}{l}\theta_{p} \\
\text { deg }\end{array}$ & $\begin{array}{l}\theta_{0} \\
\text { deg }\end{array}$ & $\sigma$ & V & $\delta$ & $\begin{array}{l}\Delta \theta \\
\operatorname{deg}\end{array}$ & $A$ \\
\hline 940922 & 0500 & 1.30 & 0.093 & 10.7 & 44 & 72 & 0.71 & 1.62 & 3.64 & 45 & 0.18 \\
\hline 940922 & 0800 & 1.25 & 0.093 & 10.7 & 44 & 74 & 0.77 & 1.44 & 3.00 & 53 & 0.41 \\
\hline 940923 & 1028 & 1.14 & 0.074 & 13.6 & 40 & 90 & 0.87 & 0.53 & 2.00 & 92 & 0.46 \\
\hline 940923 & 1401 & 1.06 & 0.074 & 13.6 & 38 & 98 & 0.90 & 0.32 & 2.02 & 92 & 0.27 \\
\hline 940923 & 1700 & 1.06 & 0.074 & 13.6 & 78 & 98 & 0.83 & 0.49 & 2.04 & 79 & 0.88 \\
\hline 940923 & 2000 & 1.09 & 0.074 & 13.6 & 58 & 94 & 0.87 & 0.64 & 1.87 & 99 & 1.02 \\
\hline 940924 & 2000 & 1.07 & 0.074 & 13.6 & 50 & 90 & 0.87 & 0.74 & 2.06 & 90 & 0.75 \\
\hline 940925 & 0500 & 1.02 & 0.083 & 12.0 & 76 & 99 & 0.89 & 0.39 & 1.80 & 94 & 0.71 \\
\hline 940925 & 0800 & 1.06 & 0.083 & 12.0 & 48 & 93 & 0.90 & 0.68 & 1.79 & 102 & 0.94 \\
\hline 940925 & 1058 & 1.00 & 0.083 & 12.0 & 60 & 90 & 0.84 & 0.82 & 2.10 & 87 & 1.15 \\
\hline 940925 & 1700 & 1.04 & 0.083 & 12.0 & 46 & 97 & 0.91 & 0.47 & 1.76 & 101 & 0.56 \\
\hline 940925 & 2000 & 0.98 & 0.083 & 12.0 & 52 & 96 & 0.88 & 0.68 & 1.96 & 93 & 0.81 \\
\hline 940925 & 2300 & 0.92 & 0.083 & 12.0 & 52 & 97 & 0.87 & 0.52 & 1.93 & 91 & 0.60 \\
\hline 940926 & 0200 & 0.85 & 0.083 & 12.0 & 60 & 103 & 0.89 & 0.53 & 1.99 & 89 & 0.60 \\
\hline 940926 & 0500 & 0.91 & 0.083 & 12.0 & 42 & 98 & 0.89 & 0.42 & 1.94 & 92 & 0.30 \\
\hline 940926 & 0800 & 0.97 & 0.054 & 18.5 & 64 & 87 & 0.80 & 1.08 & 2.69 & 67 & 1.09 \\
\hline 940926 & 1700 & 1.40 & 0.054 & 18.5 & 62 & 80 & 0.80 & 1.91 & 3.19 & 58 & 1.73 \\
\hline 940926 & 2000 & 1.70 & 0.064 & 15.6 & 64 & 86 & 0.87 & 1.53 & 2.41 & 89 & 1.98 \\
\hline 940926 & 2300 & 1.53 & 0.064 & 15.6 & 56 & 78 & 0.84 & 2.54 & 3.14 & 61 & 2.17 \\
\hline 940927 & 0200 & 1.63 & 0.064 & 15.6 & 60 & 83 & 0.88 & 1.90 & 2.63 & 83 & 2.62 \\
\hline 940927 & 0500 & 1.53 & 0.064 & 15.6 & 58 & 95 & 1.01 & 1.11 & 1.78 & 125 & 2.17 \\
\hline 940927 & 0800 & 1.69 & 0.074 & 13.6 & 54 & 84 & 0.95 & 1.70 & 2.25 & 113 & 2.30 \\
\hline 940927 & 1100 & 1.75 & 0.074 & 13.6 & 54 & 92 & 1.03 & 1.09 & 1.81 & 130 & 1.51 \\
\hline 940927 & 1401 & 1.82 & 0.074 & 13.6 & 54 & 76 & 0.88 & 2.35 & 2.97 & 69 & 1.84 \\
\hline 940927 & 1701 & 1.88 & 0.074 & 13.6 & 48 & 92 & 0.99 & 0.99 & 1.95 & 114 & 1.16 \\
\hline 940927 & 2300 & 1.64 & 0.083 & 12.0 & 48 & 78 & 0.89 & 1.57 & 2.58 & 85 & 1.25 \\
\hline 940928 & 0200 & 1.46 & 0.083 & 12.0 & 50 & 78 & 0.91 & 1.87 & 2.59 & 88 & 1.71 \\
\hline 940928 & 0500 & 1.47 & 0.083 & 12.0 & 48 & 76 & 0.85 & 1.76 & 2.90 & 64 & 0.99 \\
\hline 940928 & 1055 & 1.40 & 0.093 & 10.7 & 48 & 72 & 0.85 & 2.15 & 2.99 & 60 & 1.33 \\
\hline 940928 & 1400 & 1.22 & 0.093 & 10.7 & 46 & 74 & 0.85 & 1.76 & 2.76 & 68 & 1.27 \\
\hline 940928 & 1700 & 1.10 & 0.093 & 10.7 & 48 & 87 & 0.93 & 0.99 & 1.99 & 105 & 1.14 \\
\hline 940928 & 1957 & 1.11 & 0.093 & 10.7 & 46 & 77 & 0.89 & 1.54 & 2.45 & 88 & 1.31 \\
\hline 940929 & 0200 & 0.98 & 0.093 & 10.7 & 48 & 86 & 0.88 & 1.19 & 2.28 & 99 & 1.00 \\
\hline 940929 & 0500 & 0.94 & 0.093 & 10.7 & 46 & 80 & 0.85 & 1.25 & 2.51 & 75 & 0.89 \\
\hline 940929 & 0757 & 0.95 & 0.093 & 10.7 & 48 & 78 & 0.82 & 1.36 & 2.64 & 65 & 0.84 \\
\hline 940929 & 1900 & 0.88 & 0.103 & 9.7 & 42 & 81 & 0.84 & 0.97 & 2.40 & 76 & 0.36 \\
\hline 940929 & 1357 & 0.81 & 0.093 & 10.7 & 48 & 88 & 0.91 & 0.78 & 2.04 & 97 & 0.62 \\
\hline 940929 & 1700 & 0.78 & 0.093 & 10.7 & 44 & 94 & 0.91 & 0.52 & 1.97 & 95 & 0.27 \\
\hline 940929 & 2000 & 0.78 & 0.064 & 15.6 & 46 & 98 & 0.92 & 0.47 & 1.87 & 101 & 0.38 \\
\hline 940930 & 0200 & 0.76 & 0.064 & 15.6 & 50 & 105 & 0.91 & 0.36 & 1.85 & 99 & 0.46 \\
\hline 940930 & 0500 & 0.82 & 0.162 & 6.2 & 54 & 93 & 0.87 & 0.66 & 2.09 & 87 & 0.48 \\
\hline 940930 & 0800 & 0.94 & 0.162 & 6.2 & 50 & 79 & 0.85 & 1.30 & 2.48 & 74 & 1.20 \\
\hline 940930 & 1400 & 1.04 & 0.162 & 6.2 & 40 & 69 & 0.90 & 1.43 & 2.46 & 82 & 1.14 \\
\hline 940930 & 1700 & 0.96 & 0.162 & 6.2 & 54 & 73 & 0.88 & 1.14 & 2.42 & 77 & 0.79 \\
\hline 940930 & 1954 & 1.10 & 0.162 & 6.2 & 42 & 57 & 0.82 & 2.30 & 3.45 & 55 & 1.22 \\
\hline 941001 & 0159 & 0.90 & 0.152 & & 48 & 76 & 0.84 & 1.56 & 2.69 & 68 & 1.33 \\
\hline 941001 & 0459 & 0.86 & 0.152 & 6.6 & 48 & 75 & 0.87 & 1.38 & 2.55 & 74 & 1.24 \\
\hline 941001 & 1059 & 0.89 & 0.142 & 7.0 & 40 & 71 & 0.94 & 1.54 & 2.32 & 99 & 1.73 \\
\hline 941001 & 1358 & 0.91 & 0.152 & 6.6 & 42 & 72 & 0.93 & 1.63 & 2.36 & 100 & 1.70 \\
\hline 941001 & 1659 & 0.90 & 0.152 & 6.6 & 34 & 70 & 0.95 & 1.65 & 2.34 & 104 & 1.66 \\
\hline 941001 & 1959 & 0.81 & 0.064 & 15.6 & 42 & 87 & 0.99 & 0.89 & 1.80 & 195 & 1.11 \\
\hline & & & & & & & & & & & \\
\hline
\end{tabular}




\begin{tabular}{|c|c|c|c|c|c|c|c|c|c|c|c|}
\hline Date & $\begin{array}{l}\text { Time } \\
\text { GMT }\end{array}$ & $\begin{array}{l}H_{m o} \\
\mathbf{m}\end{array}$ & $\begin{array}{l}f_{p} \\
\mathrm{~Hz}\end{array}$ & $\begin{array}{l}T_{p} \\
\mathrm{sec}\end{array}$ & $\begin{array}{c}\theta_{p} \\
\text { deg }\end{array}$ & $\begin{array}{l}\theta_{0} \\
\text { deg }\end{array}$ & $\sigma$ & Y & $\delta$ & $\begin{array}{c}\Delta \theta \\
\text { deg }\end{array}$ & A \\
\hline 941002 & 0159 & 0.87 & 0.123 & 8.2 & 46 & 71 & 0.87 & 2.01 & 2.74 & 77 & 2.05 \\
\hline 941002 & 0459 & 0.81 & 0.132 & 7.6 & 44 & 83 & 1.00 & 1.27 & 1.93 & 118 & 2.04 \\
\hline 941002 & 0756 & 0.75 & 0.064 & 15.6 & 46 & 101 & 1.00 & 0.42 & 1.56 & 120 & 0.46 \\
\hline 941002 & 1359 & 0.81 & 0.064 & 15.6 & 46 & 98 & 1.02 & 0.59 & 1.61 & 125 & 0.64 \\
\hline 941002 & 1658 & 0.79 & 0.074 & 13.6 & 48 & 97 & 1.01 & 0.59 & 1.60 & 116 & 0.79 \\
\hline 941002 & 1956 & 0.87 & 0.074 & 13.6 & 52 & 96 & 1.01 & 0.69 & 1.57 & 120 & 1.62 \\
\hline 941003 & 0759 & 1.05 & 0.113 & 8.9 & 46 & 69 & 0.89 & 2.37 & 2.90 & 71 & 2.09 \\
\hline 941003 & 1104 & 1.10 & 0.113 & 8.9 & 44 & 66 & 0.83 & 2.19 & 3.08 & 62 & 2.03 \\
\hline 941003 & 1345 & 1.22 & 0.113 & 8.9 & 40 & 59 & 0.79 & 2.27 & 3.52 & 52 & 1.24 \\
\hline 941003 & 1659 & 1.09 & 0.113 & 8.9 & 44 & 63 & 0.86 & 2.26 & 3.17 & 62 & 1.63 \\
\hline 941003 & 1956 & 1.18 & 0.103 & 9.7 & 46 & 70 & 0.87 & 1.92 & 2.81 & 68 & 1.61 \\
\hline 941003 & 2258 & 1.34 & 0.093 & 10.7 & 44 & 63 & 0.78 & 2.52 & 3.84 & 45 & 1.76 \\
\hline 941004 & 0159 & 1.36 & 0.093 & 10.7 & 44 & 63 & 0.72 & 2.31 & 4.02 & 41 & 1.37 \\
\hline 941004 & 0459 & 1.15 & 0.093 & 10.7 & 48 & 67 & 0.78 & 2.01 & 3.51 & 51 & 1.54 \\
\hline 941004 & 0803 & 1.17 & 0.103 & 9.7 & 48 & 72 & 0.79 & 1.73 & 3.24 & 56 & 1.26 \\
\hline 941004 & 0951 & 1.24 & 0.103 & 9.7 & 50 & 72 & 0.74 & 1.84 & 3.64 & 46 & 1.19 \\
\hline 941004 & 1151 & 1.21 & 0.103 & 9.7 & 48 & 75 & 0.74 & 1.70 & 3.58 & 49 & 0.75 \\
\hline 941004 & 1319 & 1.25 & 0.103 & 9.7 & 48 & 75 & 0.75 & 1.69 & 3.40 & 52 & 0.89 \\
\hline 941006 & 1520 & 1.34 & 0.083 & 12.0 & 54 & 79 & 0.68 & 0.78 & 3.34 & 50 & -0.03 \\
\hline 941006 & 1723 & 1.53 & 0.162 & 6.2 & 56 & 70 & 0.63 & 0.65 & 3.66 & 46 & 0.30 \\
\hline 941006 & 2123 & 1.74 & 0.083 & 12.0 & 58 & 79 & 0.59 & 0.42 & 3.66 & 45 & 0.39 \\
\hline 941006 & 2323 & 1.81 & 0.083 & 12.0 & 58 & 74 & 0.55 & 0.34 & 4.13 & 41 & 0.15 \\
\hline 941007 & 0123 & 1.82 & 0.083 & 12.0 & 58 & 73 & 0.55 & 0.50 & 4.52 & 39 & 0.23 \\
\hline 941007 & 0323 & 1.85 & 0.132 & 7.6 & 54 & 70 & 0.56 & 0.6 & 4.36 & 42 & 0.36 \\
\hline 941007 & 0523 & 1.77 & 0.083 & 12.0 & 54 & 66 & 0.55 & 0.85 & 4.85 & 39 & 0.60 \\
\hline 941007 & 0723 & 1.64 & 0.093 & 10.7 & 54 & 64 & 0.57 & 0.97 & 4.70 & 38 & 0.66 \\
\hline 941007 & 0920 & 1.53 & 0.103 & 9.7 & 52 & 64 & 0.60 & 1.12 & 4.42 & 40 & 1.02 \\
\hline 941007 & 1123 & 1.66 & 0.093 & 10.7 & 52 & 62 & 0.58 & 1.30 & 4.99 & 34 & 1.02 \\
\hline 941007 & 1323 & 1.65 & 0.093 & 10.7 & 46 & 58 & 0.58 & 1.61 & 5.34 & 32 & 0.95 \\
\hline 941007 & 1523 & 1.64 & 0.103 & 9.7 & 42 & 56 & 0.59 & 1.41 & 4.43 & 38 & 0.99 \\
\hline 941007 & 1723 & 1.74 & 0.103 & 9.7 & 42 & 55 & 0.57 & 1.3 & 4.5 & 39 & 0.81 \\
\hline 941007 & 1923 & 1.74 & 0.103 & 9.7 & 48 & 59 & 0.56 & 1.02 & 4.23 & 43 & 0.79 \\
\hline 941007 & 2120 & 1.53 & 0.103 & 9.7 & 50 & 62 & 0.59 & 1.11 & 4.49 & 40 & 0.96 \\
\hline 941007 & 2323 & 1.43 & 0.103 & 9.7 & 48 & 63 & 0.61 & 1.00 & 4.05 & 43 & 0.83 \\
\hline 941008 & 0123 & 1.36 & 0.103 & 9.7 & 50 & 61 & 0.58 & 1.22 & 4.72 & 36 & 0.77 \\
\hline 941008 & 0723 & 1.23 & 0.113 & 8.9 & 46 & 59 & 0.62 & 1.84 & 4.70 & 32 & 0.89 \\
\hline 941008 & 0923 & 1.20 & 0.103 & 9.7 & 44 & 53 & 0.58 & 2.3 & 5.81 & 25 & 0.54 \\
\hline 941008 & 1123 & 1.21 & 0.103 & 9.7 & 40 & 53 & 0.59 & 2.39 & 5.67 & 25 & 0.74 \\
\hline 941008 & 1325 & 1.26 & 0.113 & 8.9 & 40 & 53 & 0.60 & 2.29 & 5.41 & 26 & 0.84 \\
\hline 941008 & 1523 & 9.25 & 0.113 & 8.9 & 42 & 56 & 0.62 & 1.5 & 4.81 & 32 & 0.98 \\
\hline 941008 & 1723 & 1.18 & 0.123 & 8.2 & 44 & 56 & 0.63 & 1.72 & 4.41 & 37 & 0.85 \\
\hline 941008 & 1923 & 1.09 & 0.113 & 8.9 & 44 & 56 & 0.66 & 1.7 & 4.16 & 39 & 0.85 \\
\hline 941008 & 2119 & 1.02 & 0.113 & 8.9 & 46 & 57 & 0.69 & 1.64 & 3.84 & 41 & 0.72 \\
\hline 941008 & 2323 & 1.01 & 0.113 & 8.9 & 50 & 61 & 0.70 & 1.63 & 3.62 & 40 & 0.88 \\
\hline 941009 & 0123 & 1.00 & 0.074 & 13.6 & 50 & 64 & 0.69 & 9.52 & 3.56 & 42 & 1.02 \\
\hline 941009 & 0323 & & 0.074 & & 48 & 63 & 0.67 & 1.8 & 3.96 & 37 & 1.18 \\
\hline 941009 & 0523 & 1.02 & 0.074 & 13.6 & 44 & 63 & 0.71 & 1.76 & 3.54 & 46 & 1.48 \\
\hline 941009 & 0723 & 1.09 & 0.074 & 13.6 & 48 & 64 & 0.72 & 9.85 & 3.47 & 45 & 1.31 \\
\hline 941009 & 0920 & 1.17 & 0.074 & 13.6 & 46 & 62 & 0.74 & 1.83 & 3.39 & 47 & 1.46 \\
\hline 941009 & 1123 & 1.23 & 0.074 & 13.6 & 48 & 61 & 0.68 & 2.23 & 4.07 & 28 & 1.38 \\
\hline 941009 & 1754 & 1.20 & 0.074 & 13.6 & 50 & 65 & 0.74 & 1.58 & 3.21 & 51 & 1.23 \\
\hline 941009 & 2123 & 1.15 & 0.074 & 13.6 & 50 & 70 & 0.79 & 1.36 & 2.83 & 64 & 1.21 \\
\hline 941009 & 2323 & 1.13 & 0.074 & 13.6 & 48 & 70 & 0.78 & 1.40 & 2.84 & 66 & 1.64 \\
\hline \multicolumn{12}{|c|}{ (Sheet 33 of 45 ) } \\
\hline
\end{tabular}




\begin{tabular}{|c|c|c|c|c|c|c|c|c|c|c|c|}
\hline Date & $\begin{array}{l}\text { Time } \\
\text { GMT }\end{array}$ & $\begin{array}{l}H_{m 0} \\
\mathrm{~m}\end{array}$ & $\begin{array}{l}f_{p} \\
\mathrm{~Hz}\end{array}$ & $\begin{array}{l}T_{p} \\
\mathrm{sec}\end{array}$ & $\begin{array}{l}\theta_{p} \\
\text { deg }\end{array}$ & $\begin{array}{l}\theta_{0} \\
\text { deg }\end{array}$ & $\sigma$ & $Y$ & $\delta$ & $\begin{array}{l}\Delta \theta \\
\text { deg }\end{array}$ & A \\
\hline 941010 & 0123 & 1.18 & 0.074 & 13.6 & 50 & 71 & 0.77 & 1.33 & 2.84 & 68 & 1.68 \\
\hline 941010 & 0323 & 1.17 & 0.074 & 13.6 & 48 & 77 & 0.83 & 0.86 & 2.17 & 86 & 1.84 \\
\hline 941010 & 0523 & 1.10 & 0.083 & 12.0 & 44 & 78 & 0.82 & 0.80 & 2.18 & 86 & 1.44 \\
\hline 941010 & 0723 & 1.09 & 0.083 & 12.0 & 46 & 80 & 0.81 & 0.74 & 2.08 & 85 & 1.50 \\
\hline 941010 & 1058 & 1.08 & 0.083 & 12.0 & 50 & 92 & 0.81 & 0.36 & 1.94 & 85 & 0.41 \\
\hline 941010 & 1357 & 1.11 & 0.064 & 15.6 & 144 & 94 & 0.82 & 0.23 & 1.89 & 88 & 0.47 \\
\hline 941010 & 1657 & 1.10 & 0.064 & 15.6 & 48 & 90 & 0.84 & 0.34 & 1.92 & 90 & 0.49 \\
\hline 941010 & 1955 & 1.14 & 0.064 & 15.6 & 52 & 84 & 0.84 & 0.39 & 2.01 & 85 & 0.68 \\
\hline 941011 & 0157 & 1.28 & 0.162 & 6.2 & 52 & 72 & 0.80 & 0.90 & 2.50 & 71 & 1.11 \\
\hline 941011 & 0457 & 1.61 & 0.152 & 6.6 & 48 & 65 & 0.70 & 1.42 & 3.32 & 44 & 0.71 \\
\hline 941011 & 0757 & 1.79 & 0.142 & 7.0 & 56 & 58 & 0.63 & 1.51 & 4.28 & 38 & 0.07 \\
\hline 941011 & 1057 & 1.76 & 0.132 & 7.6 & 52 & 61 & 0.63 & 1.82 & 4.50 & 31 & 0.52 \\
\hline 941011 & 1357 & 1.60 & 0.083 & 12.0 & 52 & 63 & 0.64 & 1.96 & 4.44 & 28 & 0.78 \\
\hline 941011 & 1657 & 1.82 & 0.093 & 10.7 & 54 & 62 & 0.65 & 1.82 & 4.20 & 30 & 0.41 \\
\hline 941011 & 1956 & 2.32 & 0.093 & 10.7 & 48 & 52 & 0.54 & 1.79 & 5.91 & 27 & 0.08 \\
\hline 941012 & 0157 & 2.27 & 0.103 & 9.7 & 50 & 58 & 0.53 & 1.70 & 5.56 & 27 & 0.46 \\
\hline 941012 & 0457 & 2.28 & 0.103 & 9.7 & 52 & 58 & 0.55 & 1.52 & 5.48 & 30 & 0.32 \\
\hline 941012 & 0757 & 2.88 & 0.113 & 8.9 & 56 & 54 & 0.46 & 1.22 & 6.49 & 27 & -0.14 \\
\hline 941012 & 1057 & 3.24 & 0.113 & 8.9 & 50 & 51 & 0.42 & 1.10 & 7.01 & 26 & 0.02 \\
\hline 941012 & 1357 & 3.64 & 0.103 & 9.7 & 50 & 49 & 0.49 & 0.97 & 7.05 & 25 & -0.11 \\
\hline 941012 & 1657 & 3.46 & 0.113 & 8.9 & 48 & 47 & 0.44 & 1.02 & 6.60 & 29 & -0.05 \\
\hline 941012 & 1955 & 3.57 & 0.103 & 9.7 & 46 & 46 & 0.42 & 0.92 & 6.59 & 28 & 0.06 \\
\hline 941013 & 0158 & 3.15 & 0.113 & 8.9 & 50 & 48 & 0.45 & 0.91 & 6.06 & 30 & -0.15 \\
\hline 941013 & 0457 & 2.88 & 0.103 & 9.7 & 48 & 51 & 0.45 & 1.09 & 6.46 & 27 & 0.25 \\
\hline 941013 & 0757 & 2.81 & 0.113 & 8.9 & 48 & 50 & 0.43 & 1.56 & 8.18 & 23 & 0.07 \\
\hline 941013 & 1057 & 2.66 & 0.103 & 9.7 & 44 & 52 & 0.44 & 1.50 & 6.97 & 26 & 0.36 \\
\hline 941013 & 1355 & 2.61 & 0.103 & 9.7 & 48 & 55 & 0.44 & 1.07 & 6.50 & 28 & 0.40 \\
\hline 941013 & 1658 & 3.30 & 0.103 & 9.7 & 50 & 54 & 0.42 & 0.87 & 6.53 & 26 & 0.22 \\
\hline 941013 & 2002 & 3.35 & 0.093 & 10.7 & 48 & 51 & 0.40 & 1.14 & 7.75 & 21 & 0.22 \\
\hline 941014 & 0202 & 3.44 & 0.093 & 10.7 & 50 & 55 & 0.38 & 1.00 & 8.04 & 22 & 0.22 \\
\hline 941014 & 0502 & 3.27 & 0.093 & 10.7 & 48 & 54 & 0.38 & 1.32 & 8.98 & 20 & 0.45 \\
\hline 941014 & 0802 & 3.09 & 0.093 & 10.7 & 48 & 53 & 0.40 & 1.60 & 10.04 & 19 & 0.46 \\
\hline 941014 & 1059 & 2.51 & 0.093 & 10.7 & 50 & 55 & 0.42 & 1.60 & 8.74 & 20 & 0.36 \\
\hline 941014 & 1403 & 2.35 & 0.103 & 9.7 & 52 & 57 & 0.45 & 1.40 & 7.20 & 24 & 0.39 \\
\hline 941014 & 1700 & 2.50 & 0.103 & 9.7 & 52 & 56 & 0.44 & 0.91 & 6.50 & 27 & 0.23 \\
\hline 941014 & 2002 & 3.14 & 0.113 & 8.9 & 50 & 57 & 0.41 & 0.67 & 5.93 & 27 & 0.32 \\
\hline 941015 & 0204 & 4.38 & 0.103 & 9.7 & 52 & 52 & 0.40 & 0.42 & 4.90 & 29 & -0.03 \\
\hline 941015 & 0502 & 4.56 & 0.093 & 10.7 & 50 & 51 & 0.39 & 0.47 & 5.54 & 28 & 0.03 \\
\hline 941015 & 0805 & 4.30 & 0.083 & 12.0 & 46 & 50 & 0.37 & 0.86 & 8.03 & 21 & 0.32 \\
\hline 941015 & 1102 & 4.10 & 0.093 & 10.7 & 48 & 51 & 0.37 & 0.89 & 8.44 & 20 & 0.23 \\
\hline 941015 & 1404 & 3.96 & 0.093 & 10.7 & 46 & 52 & 0.37 & 0.71 & 6.86 & 24 & 0.36 \\
\hline 941015 & 1704 & 4.38 & 0.093 & 10.7 & 48 & 52 & 0.36 & 0.77 & 8.42 & 21 & 0.30 \\
\hline 941015 & 2002 & 4.12 & 0.093 & 10.7 & 50 & 53 & 0.37 & 0.70 & 7.98 & 22 & 0.21 \\
\hline 941016 & 0202 & 3.18 & 0.093 & 10.7 & 50 & 56 & 0.36 & 1.14 & 10.51 & 20 & 0.33 \\
\hline 941016 & 0505 & 2.91 & 0.093 & 10.7 & 48 & 55 & 0.35 & 1.45 & 11.29 & 18 & 0.51 \\
\hline 941016 & 0805 & 2.70 & 0.093 & 10.7 & 48 & 56 & 0.41 & 1.51 & 9.16 & 19 & 0.59 \\
\hline 941016 & 1104 & 2.29 & 0.093 & 10.7 & 50 & 63 & 0.50 & 1.16 & 5.84 & 33 & 1.26 \\
\hline 941016 & 1404 & 2.42 & 0.093 & 10.7 & 50 & 61 & 0.44 & 1.40 & 7.40 & 26 & 1.09 \\
\hline 941016 & 1705 & 2.09 & 0.103 & 9.7 & 50 & 58 & 0.44 & 1.56 & 7.86 & 23 & 0.81 \\
\hline 941016 & 2005 & 1.92 & 0.103 & 9.7 & 52 & 60 & 0.45 & 1.79 & 9.22 & 19 & 0.62 \\
\hline 941017 & 0205 & 1.81 & 0.083 & 12.0 & 50 & 61 & 0.48 & 1.96 & 7.40 & 22 & 0.88 \\
\hline & 0504 & 1.77 & 0.093 & 10.7 & 52 & 61 & 0.49 & 1.88 & 7.75 & 24 & 0.77 \\
\hline 941017 & 0804 & 1.62 & 0.103 & 9.7 & 52 & 62 & 0.51 & 1.58 & 6.92 & 26 & 0.90 \\
\hline \multicolumn{12}{|c|}{ (Sheet 34 of 45 ) } \\
\hline
\end{tabular}




\begin{tabular}{|c|c|c|c|c|c|c|c|c|c|c|c|}
\hline \multicolumn{12}{|c|}{ Table A1 (Continued) } \\
\hline Date & $\begin{array}{l}\text { Time } \\
\text { GMT }\end{array}$ & $\begin{array}{l}H_{m 0} \\
\mathrm{~m}\end{array}$ & $\begin{array}{l}f_{p} \\
H_{2}\end{array}$ & $\begin{array}{l}T_{p} \\
\text { sec }\end{array}$ & $\begin{array}{l}\theta_{p} \\
\text { deg }\end{array}$ & $\begin{array}{l}\theta_{0} \\
\operatorname{deg}\end{array}$ & $\sigma$ & $\mathbf{Y}$ & $\delta$ & $\begin{array}{l}\Delta \theta \\
\operatorname{deg}\end{array}$ & $A$ \\
\hline 941017 & 1057 & 1.43 & 0.103 & 9.7 & 50 & 69 & 0.59 & 1.01 & 4.38 & 43 & 1.08 \\
\hline 941017 & 1404 & 1.65 & 0.103 & 9.7 & 50 & 67 & 0.51 & 1.17 & 5.44 & 35 & 0.96 \\
\hline 941017 & 1704 & 1.50 & 0.103 & 9.7 & 54 & 65 & 0.53 & 1.66 & 6.60 & 27 & 0.55 \\
\hline 941017 & 2004 & 1.34 & 0.064 & 15.6 & 54 & 70 & 0.60 & 1.50 & 5.42 & 33 & 0.38 \\
\hline 941018 & 0202 & 1.53 & 0.064 & 15.6 & 46 & 70 & 0.61 & 1.16 & 4.53 & 41 & 0.04 \\
\hline 941018 & 0504 & 1.60 & 0.064 & 15.6 & 46 & 68 & 0.60 & 1.17 & 4.60 & 43 & 0.39 \\
\hline 941018 & 0805 & 1.53 & 0.064 & 15.6 & 50 & 71 & 0.62 & 1.43 & 4.79 & 37 & 0.20 \\
\hline 941018 & 1102 & 1.50 & 0.064 & 15.6 & 90 & 87 & 0.62 & 0.80 & 4.13 & 44 & -0.09 \\
\hline 941018 & 1404 & 1.54 & 0.074 & 13.6 & 64 & 84 & 0.58 & 1.33 & 5.05 & 36 & -0.05 \\
\hline 941018 & 1705 & 1.49 & 0.074 & 13.6 & 70 & 84 & 0.61 & 1.36 & 4.38 & 38 & 0.38 \\
\hline 941018 & 2005 & 1.34 & 0.074 & 13.6 & 98 & 88 & 0.72 & 0.88 & 3.17 & 50 & -0.23 \\
\hline 941019 & 0203 & 1.56 & 0.074 & 13.6 & 88 & 82 & 0.60 & 0.95 & 4.24 & 39 & -0.41 \\
\hline 941019 & 0505 & 1.70 & 0.083 & 12.0 & 52 & 74 & 0.69 & 1.68 & 3.94 & 44 & 0.64 \\
\hline 941019 & 0805 & 1.98 & 0.074 & 13.6 & 52 & 70 & 0.70 & 1.94 & 4.06 & 39 & 0.98 \\
\hline 941019 & 1104 & 2.13 & 0.074 & 13.6 & 48 & 68 & 0.66 & 1.88 & 4.25 & 40 & 1.17 \\
\hline 941019 & 1436 & 2.14 & 0.074 & 13.6 & 50 & 69 & 0.71 & 2.02 & 3.99 & 41 & 1.04 \\
\hline 941019 & 1702 & 2.15 & 0.074 & 13.6 & 50 & 62 & 0.64 & 2.32 & 5.16 & 31 & 0.75 \\
\hline 941019 & 1958 & 2.10 & 0.074 & 13.6 & 46 & 66 & 0.80 & 2.26 & 3.48 & 48 & 1.14 \\
\hline 941020 & 0158 & 1.88 & 0.074 & 13.6 & 46 & 67 & 0.74 & 1.94 & 3.60 & 49 & 1.47 \\
\hline 941020 & 0458 & 1.78 & 0.074 & 13.6 & 48 & 70 & 0.76 & 1.66 & 3.36 & 51 & 0.88 \\
\hline 941020 & 0759 & 1.62 & 0.074 & 13.6 & 50 & 70 & 0.82 & 2.23 & 3.22 & 51 & 1.53 \\
\hline 941020 & 1058 & 1.54 & 0.074 & 13.6 & 50 & 68 & 0.76 & 2.24 & 3.71 & 44 & 1.31 \\
\hline 941020 & 1355 & 1.45 & 0.083 & 12.0 & 48 & 68 & 0.77 & 1.81 & 3.48 & 49 & 1.20 \\
\hline 941020 & 1657 & 1.49 & 0.083 & 12.0 & 46 & 64 & 0.79 & 2.11 & 3.44 & 50 & 1.39 \\
\hline 941020 & 2004 & 1.35 & 0.083 & 12.0 & 46 & 72 & 0.87 & 1.78 & 2.82 & 65 & 1.01 \\
\hline 941021 & 0205 & 1.27 & 0.083 & 12.0 & 48 & 76 & 0.88 & 1.66 & 2.58 & 83 & 1.50 \\
\hline 941021 & 0504 & 1.24 & 0.093 & 10.7 & 48 & 71 & 0.85 & 2.11 & 2.95 & 58 & 1.36 \\
\hline 941021 & 0804 & 1.23 & 0.093 & 10.7 & 46 & 73 & 0.88 & 1.79 & 2.66 & 76 & 1.47 \\
\hline 941021 & 1101 & 1.15 & 0.093 & 10.7 & 50 & 82 & 0.92 & 1.31 & 2.27 & 100 & 1.36 \\
\hline 941021 & 1353 & 1.21 & 0.103 & 9.7 & 46 & 75 & 0.84 & 1.66 & 2.91 & 59 & 0.73 \\
\hline 941021 & 1651 & 1.22 & 0.103 & 9.7 & 44 & 66 & 0.76 & 1.79 & 3.52 & 50 & 0.82 \\
\hline 941021 & 1954 & 1.18 & 0.103 & 9.7 & 26 & 63 & 0.78 & 1.48 & 3.39 & 55 & 0.05 \\
\hline 941022 & 0153 & 1.22 & 0.074 & 13.6 & 52 & 72 & 0.75 & 1.70 & 3.56 & 45 & 0.58 \\
\hline 941022 & 0435 & 1.46 & 0.083 & 12.0 & 50 & 65 & 0.65 & 1.76 & 4.45 & 36 & 0.79 \\
\hline 941022 & 0754 & 1.49 & 0.083 & 12.0 & 50 & 61 & 0.65 & 2.24 & 5.01 & 33 & 0.92 \\
\hline 941022 & 1053 & 1.58 & 0.074 & 13.6 & 56 & 63 & 0.67 & 2.10 & 4.92 & 34 & 0.53 \\
\hline 941022 & 1353 & 1.47 & 0.074 & 13.6 & 52 & 68 & 0.64 & 1.68 & 4.86 & 37 & 0.80 \\
\hline 941022 & 1653 & 1.74 & 0.074 & 13.6 & 52 & 60 & 0.51 & 2.02 & 6.91 & 22 & 0.74 \\
\hline 941022 & 1954 & 1.86 & 0.074 & 13.6 & 52 & 57 & 0.54 & 1.65 & 6.56 & 24 & 0.29 \\
\hline 941023 & 0153 & 1.78 & 0.074 & 13.6 & 50 & 64 & 0.59 & 1.61 & 5.25 & 36 & 1.09 \\
\hline 941023 & 0453 & 1.92 & 0.074 & 13.6 & 50 & 61 & 0.52 & 1.60 & 6.50 & 28 & 0.86 \\
\hline 941023 & 0751 & 2.10 & 0.074 & 13.6 & 48 & 58 & 0.52 & 1.56 & 6.54 & 27 & 0.71 \\
\hline 941023 & 1353 & 2.01 & 0.083 & 12.0 & 54 & 63 & 0.50 & 1.65 & 7.31 & 24 & 0.67 \\
\hline 941023 & 1653 & 2.07 & 0.083 & 12.0 & 50 & 61 & 0.50 & 1.84 & 7.37 & 24 & 0.36 \\
\hline 941023 & 1954 & 1.98 & 0.074 & 13.6 & 52 & 62 & 0.56 & 1.46 & 5.49 & 32 & 0.72 \\
\hline 941024 & 0154 & 1.66 & 0.083 & 12.0 & 52 & 67 & 0.62 & 1.42 & 4.55 & 38 & 1.04 \\
\hline 941024 & 0452 & 1.72 & 0.074 & 13.6 & 52 & 69 & 0.64 & 1.28 & 4.12 & 43 & 0.90 \\
\hline 941024 & 0753 & 1.71 & 0.083 & 12.0 & 50 & 68 & 0.64 & 1.14 & 3.83 & 47 & 0.94 \\
\hline 941024 & 1054 & 1.65 & 0.074 & 13.6 & 52 & 71 & 0.66 & 1.03 & 3.65 & 48 & 0.78 \\
\hline 941024 & 1354 & 1.59 & 0.074 & 13.6 & 50 & 73 & 0.65 & 1.01 & 3.56 & 50 & 0.83 \\
\hline 941024 & 1650 & 1.50 & 0.083 & 12.0 & 50 & 74 & 0.66 & 0.72 & 3.26 & 53 & 0.21 \\
\hline 941024 & 1954 & 1.37 & 0.083 & 12.0 & 50 & 77 & 0.72 & 0.59 & 2.81 & 58 & 0.03 \\
\hline 941024 & 2254 & 1.17 & 0.093 & 10.7 & 48 & 82 & 0.81 & 0.56 & 2.33 & 73 & 0.07 \\
\hline
\end{tabular}




\begin{tabular}{|c|c|c|c|c|c|c|c|c|c|c|c|}
\hline \multicolumn{12}{|c|}{ Table A1 (Continued) } \\
\hline Date & $\begin{array}{l}\text { Time } \\
\text { GMT }\end{array}$ & $\begin{array}{l}H_{m o} \\
m\end{array}$ & $\begin{array}{l}f_{p} \\
\mathrm{~Hz}\end{array}$ & $\begin{array}{l}T_{p} \\
\text { sec }\end{array}$ & $\begin{array}{l}\theta_{p} \\
\text { deg }\end{array}$ & $\begin{array}{l}\theta_{0} \\
\text { deg }\end{array}$ & o & $\mathrm{Y}$ & $\delta$ & $\begin{array}{l}\Delta \theta \\
\text { deg }\end{array}$ & A \\
\hline 941025 & 0154 & 1.27 & 0.074 & 13.6 & 50 & 80 & 0.73 & 0.78 & 2.77 & 59 & 0.28 \\
\hline 941025 & 0453 & 1.27 & 0.083 & 12.0 & 42 & 77 & 0.77 & 0.63 & 2.42 & 68 & 0.23 \\
\hline 941025 & 0754 & 1.18 & 0.093 & 10.7 & 46 & 80 & 0.79 & 0.72 & 2.51 & 70 & 0.32 \\
\hline 941025 & 1053 & 1.10 & 0.083 & 12.0 & 36 & 87 & 0.82 & 0.42 & 2.15 & 85 & 0.37 \\
\hline 941025 & 1354 & 1.11 & 0.074 & 13.6 & 142 & 94 & 0.82 & 0.25 & 2.23 & 81 & 0.22 \\
\hline 941025 & 1652 & 1.03 & 0.074 & 13.6 & 140 & 103 & 0.81 & -0.08 & 2.27 & 71 & 0.25 \\
\hline 941025 & 1953 & 0.97 & 0.074 & 13.6 & 146 & 104 & 0.83 & -0.10 & 2.05 & 82 & -0.01 \\
\hline 941025 & 2254 & 1.00 & 0.074 & 13.6 & 146 & 111 & 0.82 & -0.39 & 2.10 & 77 & -0.36 \\
\hline 941026 & 0154 & 1.06 & 0.064 & 15.6 & 142 & 101 & 0.85 & -0.17 & 1.90 & 87 & -0.12 \\
\hline 941026 & 0453 & 1.15 & 0.162 & 6.2 & 144 & 92 & 0.85 & 0.21 & 1.95 & 89 & 0.33 \\
\hline 941026 & 0751 & 1.28 & 0.064 & 15.6 & 142 & 93 & 0.83 & 0.07 & 1.81 & 90 & 0.13 \\
\hline 941026 & 1354 & 1.21 & 0.064 & 15.6 & 142 & 91 & 0.83 & 0.28 & 2.01 & 88 & 0.39 \\
\hline 941026 & 1648 & 1.32 & 0.064 & 15.6 & 48 & 86 & 0.81 & 0.47 & 2.08 & 87 & 0.51 \\
\hline 941026 & 1951 & 1.59 & 0.162 & 6.2 & 40 & 74 & 0.75 & 0.70 & 2.51 & 60 & -0.10 \\
\hline 941026 & 2253 & 1.56 & 0.103 & 9.7 & 76 & 83 & 0.72 & 0.73 & 2.68 & 51 & 0.20 \\
\hline 941027 & 0154 & 1.66 & 0.103 & 9.7 & 72 & 84 & 0.63 & 0.86 & 3.67 & 37 & 0.27 \\
\hline 941027 & 0454 & 1.67 & 0.103 & 9.7 & 76 & 85 & 0.61 & 0.89 & 3.77 & 38 & 0.26 \\
\hline 941027 & 0755 & 1.80 & 0.083 & 12.0 & 54 & 81 & 0.61 & 0.82 & 3.70 & 42 & -0.01 \\
\hline 941027 & 1052 & 1.96 & 0.083 & 12.0 & 54 & 77 & 0.63 & 0.94 & 3.42 & 48 & 0.53 \\
\hline 941027 & 1353 & 1.87 & 0.083 & 12.0 & 52 & 79 & 0.61 & 0.69 & 3.58 & 47 & 0.01 \\
\hline 941027 & 1653 & 2.06 & 0.083 & 12.0 & 58 & 76 & 0.60 & 0.84 & 3.86 & 42 & 0.24 \\
\hline 941027 & 1953 & 2.08 & 0.083 & 12.0 & 54 & 73 & 0.59 & 0.56 & 3.76 & 45 & 0.18 \\
\hline 941027 & 2253 & 2.11 & 0.093 & 10.7 & 54 & 73 & 0.59 & 0.58 & 3.72 & 45 & 0.08 \\
\hline 941028 & 0154 & 2.27 & 0.083 & 12.0 & 48 & 66 & 0.57 & 0.95 & 3.89 & 43 & 0.61 \\
\hline 941028 & 0454 & 2.32 & 0.093 & 10.7 & 48 & 68 & 0.57 & 0.84 & 3.84 & 45 & 0.39 \\
\hline 941028 & 0754 & 2.35 & 0.083 & 12.0 & 50 & 64 & 0.56 & 0.98 & 4.60 & 39 & 0.60 \\
\hline 941028 & 1054 & 2.43 & 0.074 & 13.6 & 52 & 62 & 0.56 & 1.05 & 4.63 & 38 & 0.74 \\
\hline 941028 & 1353 & 2.31 & 0.074 & 13.6 & 50 & 64 & 0.59 & 1.23 & 4.54 & 40 & 0.88 \\
\hline 941028 & 1705 & 2.56 & 0.074 & 13.6 & 52 & 61 & 0.51 & 1.17 & 5.79 & 31 & 0.68 \\
\hline 941028 & 1954 & 2.85 & 0.074 & 13.6 & 50 & 60 & 0.51 & 0.85 & 5.12 & 34 & 0.57 \\
\hline 941028 & 2253 & 2.95 & 0.074 & 13.6 & 50 & 58 & 0.47 & 0.93 & 5.79 & 29 & 0.35 \\
\hline 941029 & 0151 & 2.72 & 0.074 & 13.6 & 56 & 59 & 0.46 & 0.94 & 6.06 & 27 & 0.19 \\
\hline 941029 & 0453 & 2.79 & 0.074 & 13.6 & 50 & 61 & 0.50 & 1.13 & 5.90 & 32 & 0.67 \\
\hline 941029 & 0753 & 3.18 & 0.103 & 9.7 & 50 & 59 & 0.44 & 0.73 & & 29 & 0.39 \\
\hline 941029 & 1053 & 3.04 & 0.074 & 13.6 & 48 & 56 & 0.48 & 1.10 & 5.87 & 30 & 0.60 \\
\hline 941029 & 1354 & 3.17 & 0.074 & 13.6 & 50 & 57 & 0.46 & 0.90 & 5.94 & 29 & 0.42 \\
\hline 941029 & 1653 & 3.24 & 0.093 & 10.7 & 50 & 55 & 0.46 & 0.85 & 5.91 & 29 & 0.37 \\
\hline 941029 & 1953 & 3.61 & 0.093 & 10.7 & 52 & 58 & 0.44 & 0.73 & 6.04 & 27 & 0.37 \\
\hline 941029 & 2253 & 3.58 & 0.103 & 9.7 & 52 & 56 & 0.43 & 0.63 & 5.62 & 28 & 0.20 \\
\hline 941030 & 0154 & 3.72 & 0.093 & 10.7 & 48 & 52 & 0.41 & 0.83 & 6.28 & 25 & 0.19 \\
\hline 941030 & 0451 & 3.40 & 0.093 & 10.7 & 48 & 59 & 0.44 & 0.78 & 5.85 & 29 & 0.14 \\
\hline 941030 & 0753 & 3.42 & 0.093 & 10.7 & 46 & 54 & 0.43 & 0.82 & 6.43 & 27 & 0.44 \\
\hline 941030 & 1053 & 3.14 & 0.103 & 9.7 & 48 & 55 & 0.45 & 0.62 & 5.69 & 33 & 0.39 \\
\hline 941030 & 1353 & 2.92 & 0.093 & 10.7 & 48 & 55 & 0.46 & 0.68 & 6.26 & 28 & 0.34 \\
\hline 941030 & 1654 & 2.53 & 0.103 & 9.7 & 54 & 57 & 0.48 & 0.70 & 6.37 & 29 & 0.19 \\
\hline 941030 & 1954 & 2.73 & 0.103 & 9.7 & 50 & 57 & 0.44 & 0.79 & 6.83 & 26 & 0.44 \\
\hline 941030 & 2253 & 2.52 & 0.103 & 9.7 & 50 & 59 & 0.44 & 0.67 & 6.36 & 30 & 0.33 \\
\hline 941031 & 0154 & 2.17 & 0.083 & 12.0 & 50 & 56 & 0.48 & 1.10 & 6.55 & 30 & 0.46 \\
\hline 941031 & 0451 & & 0.093 & 10.7 & 50 & 57 & 0.48 & 0.95 & 6.18 & 29 & 0.39 \\
\hline 941031 & 0753 & 2.03 & 0.083 & 12.0 & 50 & 57 & 0.49 & 1.44 & 6.36 & 26 & 0.54 \\
\hline 941031 & 1102 & 2.02 & 0.074 & 13.6 & 54 & 69 & 0.50 & 1.15 & 5.89 & 30 & 0.29 \\
\hline 941031 & 1356 & 1.81 & 0.083 & 12.0 & 54 & 62 & 0.53 & 1.79 & 6.34 & 26 & 0.51 \\
\hline 941031 & 1652 & 1.87 & 0.083 & 12.0 & 50 & 59 & 0.51 & 1.83 & 6.84 & 25 & 0.56 \\
\hline 941031 & 1954 & 1.90 & 0.083 & 12.0 & 52 & 61 & 0.48 & 2.06 & 7.75 & 21 & 0.63 \\
\hline & & & & & & & & & & & \\
\hline
\end{tabular}




\begin{tabular}{|c|c|c|c|c|c|c|c|c|c|c|c|}
\hline \multicolumn{12}{|c|}{ Table A1 (Continued) } \\
\hline Date & $\begin{array}{l}\text { Time } \\
\text { GMT }\end{array}$ & $\begin{array}{l}H_{m 0} \\
m\end{array}$ & $\begin{array}{l}f_{p} \\
\mathrm{~Hz}\end{array}$ & $\begin{array}{l}T_{p} \\
\sec \end{array}$ & $\begin{array}{l}\theta_{p} \\
\text { deg }\end{array}$ & $\begin{array}{l}\theta_{0} \\
\operatorname{deg}\end{array}$ & $\sigma$ & $\mathrm{V}$ & $\delta$ & $\begin{array}{l}\Delta \theta \\
\operatorname{deg}\end{array}$ & $A$ \\
\hline 941031 & 2255 & 1.91 & 0.083 & 12.0 & 54 & 62 & 0.53 & 1.88 & 6.40 & 25 & 0.63 \\
\hline 941101 & 0155 & 1.80 & 0.083 & 12.0 & 50 & 62 & 0.57 & 1.69 & 5.39 & 35 & 0.95 \\
\hline 941101 & 0456 & 1.71 & 0.083 & 12.0 & 50 & 63 & 0.59 & 1.81 & 4.92 & 36 & 1.15 \\
\hline 941101 & 0756 & 1.55 & 0.083 & 12.0 & 52 & 65 & 0.58 & 1.83 & 5.25 & 33 & 1.05 \\
\hline 941101 & 1121 & 1.46 & 0.083 & 12.0 & 56 & 69 & 0.60 & 1.62 & 4.76 & 36 & 0.97 \\
\hline 941101 & 1701 & 1.58 & 0.093 & 10.7 & 50 & 62 & 0.64 & 1.68 & 4.34 & 40 & 0.92 \\
\hline 941101 & 1958 & 1.62 & 0.093 & 10.7 & 46 & 59 & 0.59 & 1.79 & 4.98 & 34 & 1.10 \\
\hline 941101 & 2258 & 1.63 & 0.093 & 10.7 & 48 & 61 & 0.63 & 1.59 & 4.28 & 41 & 0.93 \\
\hline 941102 & 0200 & 2.03 & 0.152 & 6.6 & 46 & 53 & 0.53 & 1.56 & 5.38 & 28 & 0.47 \\
\hline 941102 & 0457 & 3.01 & 0.123 & 8.2 & 46 & 47 & 0.45 & 1.59 & 7.04 & 24 & 0.06 \\
\hline 941102 & 1057 & 2.94 & 0.113 & 8.9 & 48 & 50 & 0.47 & 1.22 & 6.01 & 28 & 0.05 \\
\hline 941102 & 1356 & 3.04 & 0.103 & 9.7 & 48 & 50 & 0.44 & 1.28 & 6.83 & 25 & 0.06 \\
\hline 941102 & 1700 & 3.28 & 0.103 & 9.7 & 46 & 46 & 0.45 & 1.09 & 5.84 & 29 & 0.01 \\
\hline 941102 & 2000 & 3.64 & 0.103 & 9.7 & 48 & 46 & 0.45 & 0.95 & 5.16 & 31 & -0.03 \\
\hline 941102 & 2300 & 3.71 & 0.103 & 9.7 & 44 & 48 & 0.42 & 1.24 & 6.53 & 24 & 0.20 \\
\hline 941103 & 0200 & 3.30 & 0.103 & 9.7 & 46 & 51 & 0.42 & 1.17 & 7.23 & 23 & 0.24 \\
\hline 941103 & 0457 & 3.01 & 0.093 & 10.7 & 48 & 53 & 0.42 & 1.15 & 7.31 & 25 & 0.28 \\
\hline 941103 & 0757 & 2.82 & 0.093 & 10.7 & 46 & 54 & 0.46 & 0.98 & 5.75 & 29 & 0.23 \\
\hline 941103 & 1101 & 2.98 & 0.093 & 10.7 & 44 & 52 & 0.47 & 1.42 & 6.50 & 26 & 0.44 \\
\hline 941103 & 1352 & 3.00 & 0.093 & 10.7 & 46 & 51 & 0.45 & 1.11 & 6.05 & 27 & 0.27 \\
\hline 941103 & 1656 & 2.91 & 0.093 & 10.7 & 44 & 49 & 0.44 & 9.08 & 5.90 & 28 & 0.24 \\
\hline 941103 & 1955 & 2.60 & 0.093 & 10.7 & 46 & 49 & 0.46 & 9.41 & 6.85 & 24 & 0.21 \\
\hline 941103 & 2255 & 2.26 & 0.103 & 9.7 & 46 & 50 & 0.50 & 1.26 & 5.88 & 29 & 0.16 \\
\hline 941104 & 0155 & 2.09 & 0.093 & 10.7 & 44 & 53 & 0.50 & 1.28 & 5.95 & 31 & 0.64 \\
\hline 941104 & 0453 & 1.94 & 0.093 & 10.7 & 48 & 54 & 0.49 & 1.63 & 7.17 & 25 & 0.35 \\
\hline 941104 & 0755 & 1.65 & 0.093 & 10.7 & 42 & 53 & 0.55 & 2.03 & 6.00 & 27 & 0.66 \\
\hline 941104 & 1053 & 1.50 & 0.093 & 10.7 & 44 & 56 & 0.61 & 1.79 & 5.02 & 36 & 1.15 \\
\hline 941104 & 1355 & 1.63 & 0.093 & 10.7 & 50 & 57 & 0.62 & 1.76 & 5.07 & 34 & 0.42 \\
\hline 941104 & 1708 & 1.49 & 0.103 & 9.7 & 48 & 56 & 0.59 & 1.79 & 5.31 & 31 & 0.32 \\
\hline 941104 & 1955 & 1.29 & 0.103 & 9.7 & 56 & 60 & 0.67 & 1.93 & 4.50 & 38 & 0.17 \\
\hline 941104 & 2255 & 1.20 & 0.103 & 9.7 & 50 & 68 & 0.74 & 1.46 & 3.31 & 52 & 0.69 \\
\hline 941105 & 0155 & 1.11 & 0.103 & 9.7 & 50 & 71 & 0.77 & 1.61 & 3.29 & 51 & 0.54 \\
\hline 941105 & 0455 & 1.04 & 0.064 & 15.6 & 46 & 77 & 0.81 & 1.24 & 2.65 & 67 & 0.79 \\
\hline 941105 & 0755 & 0.90 & 0.064 & 15.6 & 48 & 88 & 0.92 & 0.91 & 2.22 & 90 & 0.64 \\
\hline 941105 & 1052 & 0.93 & 0.074 & 13.6 & 52 & 82 & 0.83 & 1.05 & 2.54 & 77 & 1.00 \\
\hline 941105 & 1355 & 0.99 & 0.074 & 13.6 & 56 & 78 & 0.77 & 1.69 & 3.26 & 46 & 0.75 \\
\hline 941105 & 1955 & 1.44 & 0.093 & 10.7 & 50 & 65 & 0.6 & 2. & 4. & 36 & 0.80 \\
\hline 941105 & 2255 & 1.53 & 0.093 & 10.7 & 64 & 72 & 0.67 & 1.39 & 3.93 & 42 & 0.45 \\
\hline 941106 & 0152 & 1.73 & 0.064 & 15.6 & 50 & 68 & 0.6 & 1.73 & 5.16 & 34 & 0.54 \\
\hline 941106 & 0531 & 2.10 & 0.064 & 15.6 & 56 & 68 & 0.5 & 1.78 & 5.4 & 30 & 0.91 \\
\hline 941106 & 0755 & 2.10 & 0.064 & 15.6 & 58 & 68 & 0.5 & 2.27 & 6.02 & 29 & 0.78 \\
\hline 941106 & 1055 & 2.01 & 0.074 & 13.6 & 50 & 68 & 0.63 & 2.04 & 4.97 & 34 & 0.65 \\
\hline 941106 & 1355 & 2.21 & 0.064 & 15.6 & 50 & 65 & 0.5 & 2.11 & 5.99 & 29 & 0.70 \\
\hline 941106 & 1655 & 2.55 & 0.064 & 15.6 & 54 & 62 & 0.56 & 2.84 & 6.75 & 23 & 0.85 \\
\hline 941106 & 1955 & 2.48 & 0.064 & 15.6 & 56 & 63 & 0.56 & 2.04 & 6.19 & 28 & 0.46 \\
\hline 941106 & 2255 & 2.32 & 0.074 & 13.6 & 56 & 65 & 0.64 & 2.37 & 5.24 & 33 & 0.72 \\
\hline 941107 & 0155 & 2.55 & 0.064 & 15.6 & 56 & 65 & 0.57 & 2.58 & 6.30 & 24 & 0.62 \\
\hline & 0453 & 2.62 & 0.064 & 15.6 & 54 & 61 & 0.53 & & & 21 & 0.54 \\
\hline 941107 & 0755 & 2.56 & 0.064 & 15.6 & 56 & 63 & 0.53 & 2.47 & 7.45 & 19 & 0.43 \\
\hline 941107 & 1053 & 2.61 & 0.064 & 15.6 & 52 & 59 & 0.49 & 2.45 & 8.13 & 18 & 0.29 \\
\hline 941107 & 1353 & 2.65 & 0.074 & 13.6 & 50 & 61 & 0.51 & 9.87 & 6.87 & 25 & 0.80 \\
\hline 941107 & 1654 & 2.69 & 0.074 & 13.6 & 52 & 66 & 0.51 & 1.53 & 6.07 & 29 & 0.78 \\
\hline 941107 & 1956 & 2.97 & 0.074 & 13.6 & 52 & 62 & 0.45 & 1.71 & 8.04 & 24 & 0.50 \\
\hline
\end{tabular}




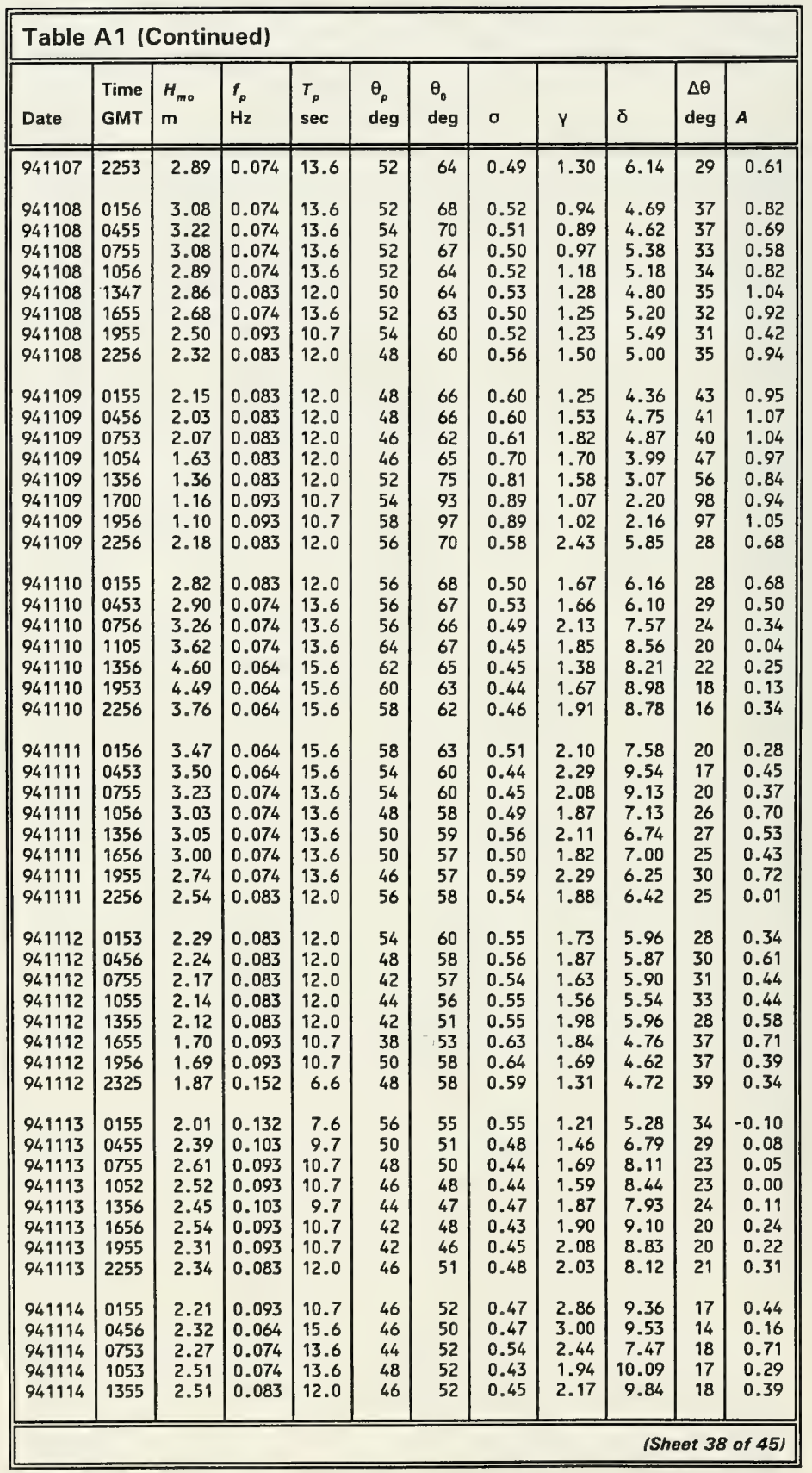




\begin{tabular}{|c|c|c|c|c|c|c|c|c|c|c|c|}
\hline \multicolumn{12}{|c|}{ Table A1 (Continued) } \\
\hline Date & $\begin{array}{l}\text { Time } \\
\text { GMT }\end{array}$ & $\begin{array}{l}H_{m o} \\
m\end{array}$ & $\begin{array}{l}f_{p} \\
\mathrm{~Hz}\end{array}$ & $\begin{array}{l}T_{p} \\
\text { sec }\end{array}$ & $\begin{array}{l}\theta_{p} \\
\text { deg }\end{array}$ & $\begin{array}{l}\theta_{0} \\
\operatorname{deg}\end{array}$ & $\sigma$ & $Y$ & $\delta$ & $\begin{array}{l}\Delta \theta \\
\operatorname{deg}\end{array}$ & A \\
\hline 941114 & 1656 & 2.07 & 0.083 & 12.0 & 44 & 54 & 0.50 & 2.20 & 7.98 & 23 & 0.55 \\
\hline 941114 & 1955 & 1.94 & 0.074 & 13.6 & 48 & 58 & 0.51 & 2.00 & 7.17 & 23 & 0.82 \\
\hline 941114 & 2255 & 1.68 & 0.074 & 13.6 & 46 & 60 & 0.61 & 2.07 & 5.46 & 33 & 1.14 \\
\hline 941115 & 0155 & 1.84 & 0.074 & 13.6 & 48 & 58 & 0.52 & 1.84 & 6.80 & 26 & 0.66 \\
\hline 941115 & 0456 & 1.88 & 0.083 & 12.0 & 46 & 55 & 0.48 & 1.97 & 8.04 & 23 & 0.71 \\
\hline 941115 & 0833 & 1.84 & 0.083 & 12.0 & 44 & 53 & 0.50 & 2.07 & 7.05 & 21 & 0.62 \\
\hline 941115 & 1053 & 1.81 & 0.083 & 12.0 & 44 & 56 & 0.54 & 1.54 & 5.83 & 32 & 1.05 \\
\hline 941115 & 1356 & 1.61 & 0.093 & 10.7 & 46 & 65 & 0.53 & 1.13 & 5.58 & 35 & 0.20 \\
\hline 941115 & 1655 & 1.30 & 0.083 & 12.0 & 52 & 65 & 0.64 & 1.56 & 4.88 & 39 & 0.44 \\
\hline 941115 & 1956 & 1.04 & 0.083 & 12.0 & 60 & 75 & 0.68 & 1.29 & 3.75 & 40 & 0.29 \\
\hline 941115 & 2256 & 1.04 & 0.083 & 12.0 & 60 & 71 & 0.57 & 1.66 & 5.53 & 29 & 0.56 \\
\hline 941116 & 0222 & 2.29 & 0.142 & 7.0 & 56 & 67 & 0.45 & 0.40 & 5.87 & 32 & 0.12 \\
\hline 941116 & 0456 & 2.73 & 0.074 & 13.6 & 58 & 63 & 0.45 & 0.14 & 4.64 & 32 & 0.08 \\
\hline 941116 & 0756 & 3.48 & 0.074 & 13.6 & 52 & 59 & 0.42 & 0.38 & 6.08 & 27 & 0.33 \\
\hline 941116 & 1052 & 4.09 & 0.074 & 13.6 & 48 & 60 & 0.39 & 0.54 & 6.44 & 27 & 0.42 \\
\hline 941116 & 1354 & 4.80 & 0.074 & 13.6 & 50 & 59 & 0.39 & 0.46 & 6.33 & 25 & 0.24 \\
\hline 941116 & 1653 & 5.69 & 0.074 & 13.6 & 48 & 53 & 0.39 & 0.48 & 5.31 & 26 & 0.28 \\
\hline 941116 & 1955 & 5.14 & 0.074 & 13.6 & 48 & 53 & 0.41 & 0.45 & 5.25 & 26 & 0.32 \\
\hline 941116 & 2255 & 4.68 & 0.074 & 13.6 & 48 & 55 & 0.43 & 0.33 & 4.97 & 31 & 0.20 \\
\hline 941117 & 0152 & 4.13 & 0.074 & 13.6 & 46 & 55 & 0.44 & 0.36 & 4.67 & 33 & 0.23 \\
\hline 941117 & 0455 & 3.99 & 0.074 & 13.6 & 48 & 54 & 0.41 & 0.48 & 5.79 & 28 & 0.23 \\
\hline 941117 & 0755 & 3.58 & 0.074 & 13.6 & 50 & 53 & 0.38 & 0.88 & 7.34 & 22 & 0.29 \\
\hline 941117 & 1104 & 3.43 & 0.083 & 12.0 & 48 & 55 & 0.41 & 0.91 & 8.00 & 24 & 0.33 \\
\hline 941117 & 1657 & 3.85 & 0.083 & 12.0 & 46 & 52 & 0.40 & 1.02 & 7.53 & 23 & 0.48 \\
\hline 941117 & 1955 & 3.08 & 0.083 & 12.0 & 44 & 52 & 0.44 & 1.17 & 6.80 & 26 & 0.47 \\
\hline 941117 & 2252 & 3.10 & 0.074 & 13.6 & 48 & 54 & 0.44 & 1.13 & 6.92 & 23 & 0.38 \\
\hline 941118 & 0154 & 3.54 & 0.083 & 12.0 & 46 & 54 & 0.44 & 0.71 & 5.38 & 30 & 0.40 \\
\hline 941118 & 0454 & 4.36 & 0.074 & 13.6 & 48 & 50 & 0.42 & 0.59 & 5.29 & 29 & 0.20 \\
\hline 941118 & 0755 & 4.66 & 0.074 & 13.6 & 44 & 47 & 0.40 & 0.77 & 6.05 & 24 & 0.15 \\
\hline 941118 & 1052 & 4.21 & 0.074 & 13.6 & 46 & 51 & 0.41 & 0.76 & 6.53 & 26 & 0.21 \\
\hline 941118 & 1355 & 4.18 & 0.083 & 12.0 & 46 & 53 & 0.41 & 0.76 & 6.95 & 26 & 0.36 \\
\hline 941118 & 1706 & 4.35 & 0.083 & 12.0 & 48 & 52 & 0.40 & 0.69 & 7.15 & 25 & 0.28 \\
\hline 941118 & 1954 & 4.05 & 0.074 & 13.6 & 48 & 50 & 0.40 & 0.90 & 7.79 & 24 & 0.17 \\
\hline 941118 & 2255 & 3.74 & 0.083 & 12.0 & 48 & 50 & 0.40 & 0.99 & 8.21 & 22 & 0.19 \\
\hline 941119 & 0155 & 3.33 & 0.083 & 12.0 & 48 & 53 & 0.41 & 0.79 & 7.41 & 26 & 0.20 \\
\hline 941119 & 0455 & 3.12 & 0.083 & 12.0 & 48 & 51 & 0.40 & 0.98 & 8.29 & 23 & 0.18 \\
\hline 941119 & 0755 & 2.93 & 0.083 & 12.0 & 46 & 49 & 0.42 & 1.3 & 8.7 & 22 & 0.13 \\
\hline 941119 & 1052 & 2.57 & 0.083 & 12.0 & 44 & 47 & 0.45 & 1.30 & $8 . ?$ & 24 & 0.09 \\
\hline 941119 & 1354 & 2.41 & 0.093 & 10.7 & 50 & 50 & 0.50 & 1.00 & 6.34 & 31 & -0.05 \\
\hline 941119 & 1655 & 2.09 & 0.083 & 12.0 & 44 & 49 & 0.51 & 1.22 & 6. & 29 & 0.21 \\
\hline 941119 & 1954 & 1.94 & 0.093 & 10.7 & 48 & 50 & 0.50 & 1.54 & 7.36 & 25 & -0.01 \\
\hline 941119 & 2255 & 1.68 & 0.093 & 10.7 & 50 & 53 & 0.57 & 1.61 & 5.90 & 31 & 0.12 \\
\hline 941120 & 0155 & 1.75 & 0.093 & 10.7 & 48 & 55 & 0.55 & 1.73 & 6.48 & 26 & 0.41 \\
\hline 941120 & 0454 & 1.79 & 0.093 & 10.7 & 46 & 51 & 0.51 & 2.01 & & 26 & 0.20 \\
\hline 941120 & 0755 & 1.59 & 0.093 & 10.7 & 46 & 54 & 0.58 & 2.07 & 6.22 & 27 & 0.36 \\
\hline 941120 & 1054 & 1.32 & 0.093 & 10.7 & 50 & 58 & 0.63 & 2.33 & 5. & 26 & 0.67 \\
\hline 941120 & 1354 & 1.30 & 0.093 & 10.7 & 46 & 55 & 0.66 & 1.88 & 4.82 & 38 & 0.57 \\
\hline 941120 & 1652 & 1.19 & 0.103 & 9.7 & 56 & 59 & 0.68 & 1.65 & 4.41 & 43 & 0.20 \\
\hline 941120 & 2021 & 1.28 & 0.162 & 6.2 & 46 & 54 & 0.60 & 1.88 & 5.63 & 33 & 0.16 \\
\hline 941120 & 2254 & 1.33 & 0.152 & 6.6 & 52 & 54 & 0.61 & 1.77 & 5.30 & 34 & 0.07 \\
\hline & & & & & & 56 & & 1.73 & & 31 & 0.17 \\
\hline 941121 & 0455 & 1.30 & 0.142 & 7.0 & 48 & 55 & 0.60 & 1.39 & 5.23 & 34 & 0.39 \\
\hline 941121 & 0754 & 1.20 & 0.162 & 6.2 & 52 & 55 & 0.62 & 1.88 & 5.67 & 34 & 0.16 \\
\hline
\end{tabular}




\begin{tabular}{|c|c|c|c|c|c|c|c|c|c|c|c|}
\hline Date & $\begin{array}{l}\text { Time } \\
\text { GMT }\end{array}$ & $\begin{array}{l}H_{m 0} \\
m\end{array}$ & $\begin{array}{l}f_{p} \\
\mathrm{~Hz}\end{array}$ & $\begin{array}{l}T_{p} \\
\text { sec }\end{array}$ & $\begin{array}{l}\theta_{p} \\
\operatorname{deg}\end{array}$ & $\begin{array}{l}\theta_{0} \\
\text { deg }\end{array}$ & $\sigma$ & $Y$ & $\delta$ & $\begin{array}{l}\Delta \theta \\
\operatorname{deg}\end{array}$ & A \\
\hline 941121 & 1054 & 1.33 & 0.152 & 6.6 & 50 & 54 & 0.58 & 1.80 & 6.25 & 32 & 0.10 \\
\hline 941121 & 1358 & 1.44 & 0.152 & 6.6 & 48 & 52 & 0.56 & 1.40 & 5.90 & 31 & 0.05 \\
\hline 941121 & 1658 & 1.81 & 0.103 & 9.7 & 46 & 50 & 0.48 & 1.15 & 6.13 & 30 & 0.06 \\
\hline 941121 & 1954 & 2.25 & 0.103 & 9.7 & 46 & 50 & 0.44 & 1.15 & 6.84 & 26 & 0.19 \\
\hline 941122 & 0155 & 2.02 & 0.093 & 10.7 & 46 & 50 & 0.44 & 2.09 & 10.02 & 18 & 0.29 \\
\hline 941122 & 0455 & 2.18 & 0.093 & 10.7 & 46 & 51 & 0.43 & 1.52 & 8.75 & 22 & 0.51 \\
\hline 941122 & 0754 & 2.27 & 0.093 & 10.7 & 46 & 50 & 0.45 & 1.55 & 8.21 & 23 & 0.40 \\
\hline 941122 & 1055 & 2.14 & 0.093 & 10.7 & 50 & 53 & 0.51 & 1.43 & 7.09 & 27 & 0.30 \\
\hline 941122 & 1355 & 1.82 & 0.103 & 9.7 & 50 & 55 & 0.50 & 2.19 & 8.40 & 21 & 0.60 \\
\hline 941122 & 1658 & 1.71 & 0.103 & 9.7 & 50 & 57 & 0.59 & 2.28 & 7.79 & 22 & 0.59 \\
\hline 941122 & 1954 & 1.69 & 0.083 & 12.0 & 48 & 56 & 0.51 & 1.94 & 7.58 & 24 & 0.52 \\
\hline 941122 & 2255 & 1.58 & 0.083 & 12.0 & 50 & 55 & 0.52 & 2.29 & 7.64 & 21 & 0.45 \\
\hline 941123 & 0152 & 1.36 & 0.083 & 12.0 & 48 & 58 & 0.56 & 2.12 & 6.43 & 25 & 0.68 \\
\hline 941123 & 0455 & 1.37 & 0.083 & 12.0 & 48 & 59 & 0.59 & 1.98 & 6.00 & 30 & 0.78 \\
\hline 941123 & 0755 & 1.41 & 0.083 & 12.0 & 46 & 57 & 0.60 & 1.97 & 5.71 & 30 & 0.51 \\
\hline 941123 & 1054 & 1.36 & 0.093 & 10.7 & 48 & 54 & 0.57 & 1.98 & 6.25 & 30 & 0.34 \\
\hline 941123 & 1404 & 1.18 & 0.093 & 10.7 & 44 & 57 & 0.68 & 2.04 & 4.58 & 39 & 0.86 \\
\hline 941123 & 1659 & 1.08 & 0.093 & 10.7 & 46 & 63 & 0.73 & 1.77 & 3.73 & 51 & 1.31 \\
\hline 941123 & 1959 & 1.06 & 0.093 & 10.7 & 48 & 65 & 0.67 & 1.34 & 4.03 & 46 & 0.64 \\
\hline 941123 & 2259 & 1.01 & 0.103 & 9.7 & 52 & 67 & 0.71 & 1.65 & 3.93 & 43 & 0.51 \\
\hline 941124 & 0159 & 0.92 & 0.074 & 13.6 & 54 & 65 & 0.72 & 1.85 & 4.00 & 41 & 0.74 \\
\hline 941124 & 0500 & 0.98 & 0.074 & 13.6 & 48 & 68 & 0.82 & 1.93 & 3.33 & 51 & 1.06 \\
\hline 941124 & 0759 & 1.17 & 0.074 & 13.6 & 48 & 64 & 0.70 & 1.55 & 3.98 & 42 & 0.45 \\
\hline 941124 & 1051 & 1.20 & 0.074 & 13.6 & 54 & 65 & 0.72 & 1.94 & 4.20 & 36 & 0.70 \\
\hline 941124 & 1359 & 1.25 & 0.054 & 18.5 & 58 & 64 & 0.66 & 2.48 & 5.24 & 26 & 0.54 \\
\hline 941124 & 1659 & 1.61 & 0.064 & 15.6 & 54 & 61 & 0.53 & 2.62 & 7.77 & 17 & 0.12 \\
\hline 941124 & 1959 & 2.01 & 0.064 & 15.6 & 56 & 59 & 0.44 & 2.37 & 10.25 & 14 & 0.12 \\
\hline 941124 & 2259 & 2.17 & 0.064 & 15.6 & 54 & 56 & 0.43 & 2.73 & 11.02 & 13 & 0.17 \\
\hline 941125 & 0159 & 2.36 & 0.064 & 15.6 & 56 & 58 & 0.42 & 2.11 & 10.36 & 12 & 0.18 \\
\hline 941125 & 0459 & 2.18 & 0.064 & 15.6 & 54 & 56 & 0.44 & 2.41 & 10.58 & 14 & 0.11 \\
\hline 941125 & 0800 & 2.07 & 0.064 & 15.6 & 58 & 57 & 0.46 & 2.03 & 9.71 & 18 & -0.17 \\
\hline 941125 & 1056 & 2.06 & 0.074 & 13.6 & 58 & 59 & 0.48 & 1.80 & 8.49 & 20 & 0.03 \\
\hline 941125 & 1359 & 1.91 & 0.074 & 13.6 & 54 & 64 & 0.52 & 1.61 & 7.01 & 27 & 0.35 \\
\hline 941125 & 1659 & 2.07 & 0.083 & 12.0 & 54 & 65 & 0.51 & 1.44 & 6.44 & 29 & 0.42 \\
\hline 941125 & 2259 & 2.03 & 0.074 & 13.6 & 52 & 64 & 0.52 & 1.23 & 5.61 & 32 & 0.58 \\
\hline 941126 & 0159 & 2.63 & 0.093 & 10.7 & 52 & 61 & 0.45 & 1.24 & 6.69 & 26 & 0.57 \\
\hline 941126 & 0459 & 3.08 & 0.093 & 10.7 & 56 & 63 & 0.41 & 0.88 & 6.8 & 25 & 0.32 \\
\hline 941126 & 0759 & 4.00 & 0.083 & 12.0 & 52 & 62 & 0.44 & 0.41 & 4.94 & 32 & 0.16 \\
\hline 941126 & 1059 & 4.70 & 0.074 & 13.6 & 56 & 60 & 0.43 & 0.33 & 4.83 & 28 & 0.01 \\
\hline 941126 & 1359 & 4.81 & 0.074 & 13.6 & 64 & 61 & 0.44 & 0.27 & 5.35 & 26 & -0.03 \\
\hline 941126 & 1659 & 4.77 & 0.064 & 15.6 & 58 & 62 & 0.45 & 0.29 & 5.09 & 29 & 0.06 \\
\hline 941126 & 1956 & 4.86 & 0.064 & 15.6 & 64 & 60 & 0.43 & 0.28 & 5.42 & 26 & -0.15 \\
\hline 941126 & 2259 & 4.66 & 0.064 & 15.6 & 58 & 60 & 0.41 & 0.53 & 6.59 & 23 & 0.18 \\
\hline 941127 & 0159 & 4.74 & 0.064 & 15.6 & 58 & 59 & 0.39 & 0.47 & 7.35 & 21 & 0.07 \\
\hline 941127 & 0459 & 4.55 & 0.074 & 13.6 & 54 & 58 & 0.40 & 0.77 & 7.60 & 22 & 0.28 \\
\hline 941127 & 0759 & 3.75 & 0.074 & 13.6 & 54 & 59 & 0.42 & 0.71 & 7.42 & 23 & 0.28 \\
\hline 941127 & 1056 & 3.08 & 0.074 & 13.6 & 54 & 58 & 0.47 & 0.85 & 6.36 & 29 & 0.20 \\
\hline 941127 & 1359 & 3.09 & 0.083 & 12.0 & 54 & 58 & 0.45 & 0.89 & 6.53 & 24 & 0.32 \\
\hline 941127 & 1657 & 2.55 & 0.074 & 13.6 & 50 & 61 & 0.53 & 1.26 & 5.48 & 30 & 0.74 \\
\hline 941127 & 1959 & 2.61 & 0.074 & 13.6 & 54 & 61 & 0.48 & 0.75 & 6.02 & 27 & 0.44 \\
\hline 941127 & 2259 & 2.67 & 0.083 & 12.0 & 52 & 57 & 0.47 & 1.07 & 6.32 & 25 & 0.39 \\
\hline 941128 & 0159 & 2.46 & 0.083 & 12.0 & 50 & 57 & 0.48 & 1.31 & 6.62 & 23 & 0.41 \\
\hline 941128 & 0459 & 2.20 & 0.083 & 12.0 & 50 & 59 & 0.52 & 1.22 & 5.64 & 31 & 0.83 \\
\hline \multicolumn{12}{|c|}{ (Sheat 40 of 45 ) } \\
\hline
\end{tabular}




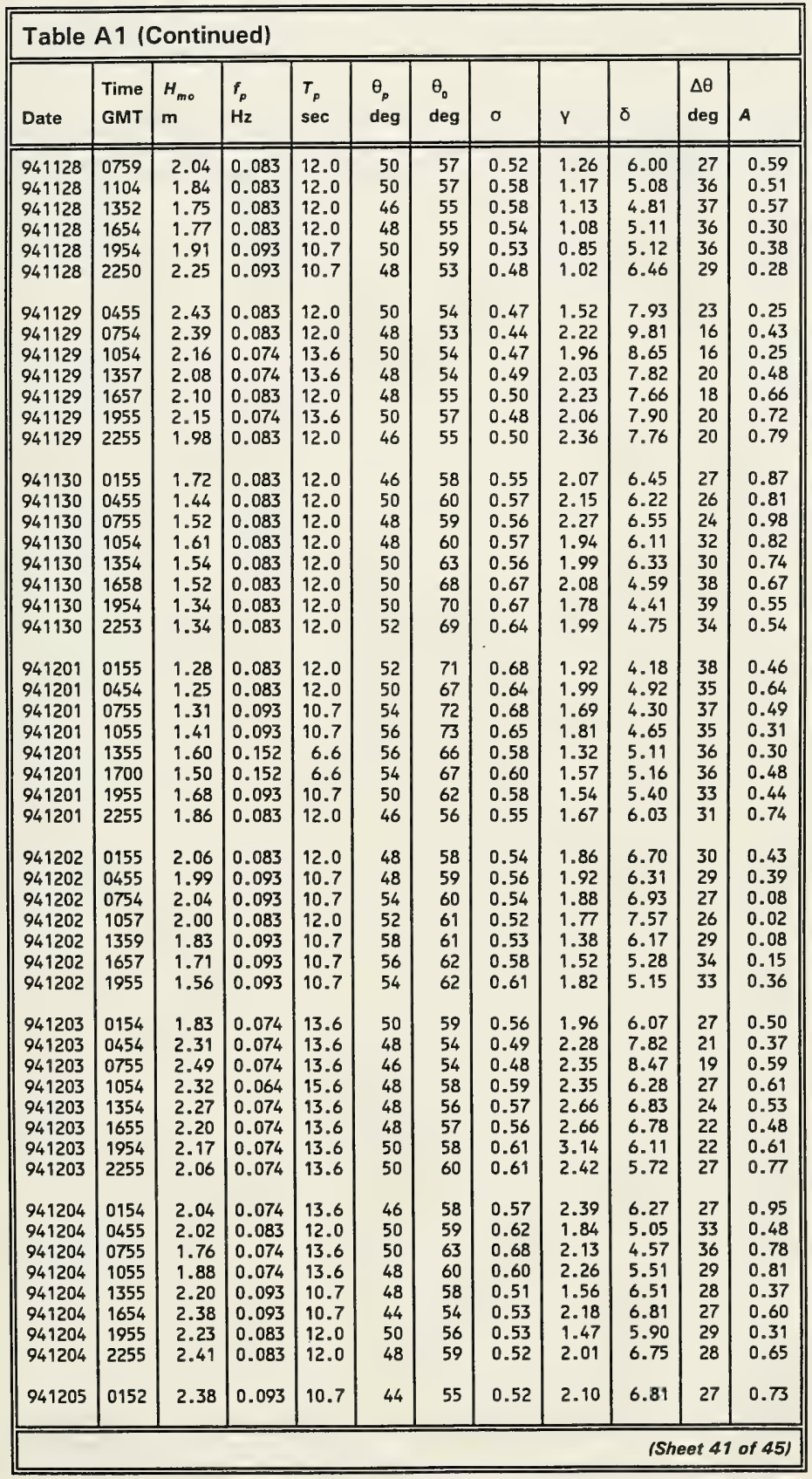




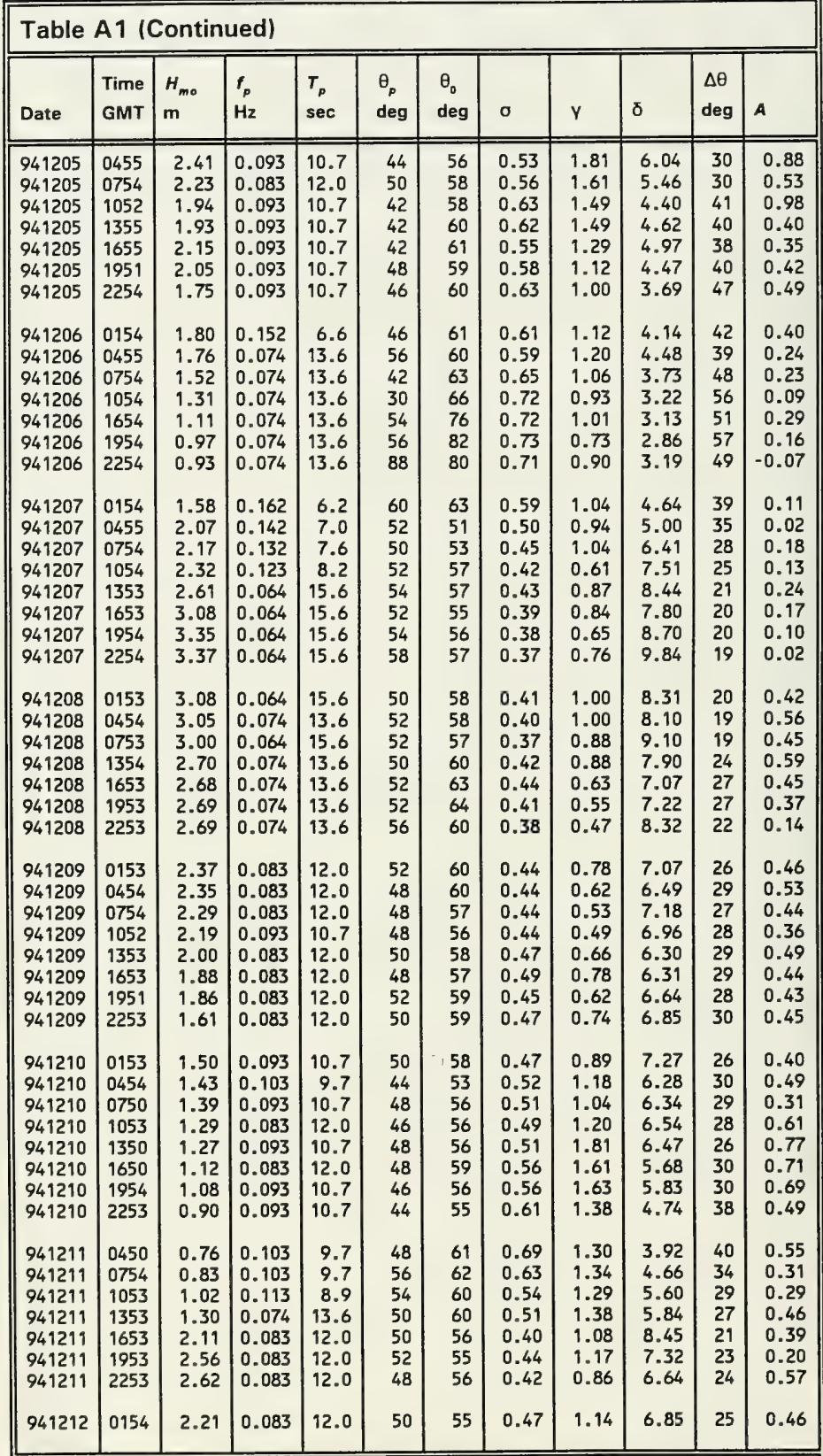

(Sheet 42 of 45) 


\begin{tabular}{|c|c|c|c|c|c|c|c|c|c|c|c|}
\hline Date & $\begin{array}{l}\text { Time } \\
\text { GMT }\end{array}$ & $\begin{array}{l}H_{m 0} \\
m\end{array}$ & $\begin{array}{l}f_{p} \\
\mathrm{~Hz}\end{array}$ & $\begin{array}{l}T_{p} \\
\mathrm{sec}\end{array}$ & $\begin{array}{l}\theta_{p} \\
\text { deg }\end{array}$ & $\begin{array}{l}\theta_{0} \\
\text { deg }\end{array}$ & $\sigma$ & $\mathrm{V}$ & $\delta$ & $\begin{array}{l}\Delta \theta \\
\operatorname{deg}\end{array}$ & A \\
\hline 941212 & 0454 & 1.78 & 0.083 & 12.0 & 50 & 58 & 0.53 & 1.27 & 6.00 & 29 & 0.49 \\
\hline 941212 & 0752 & 1.72 & .083 & 12.0 & 54 & 64 & 0.52 & 1.35 & 6.16 & 27 & 0.26 \\
\hline 941212 & 1055 & 1.67 & 0.093 & 10.7 & 56 & 63 & 0.50 & 1.45 & 6.68 & 27 & 0.33 \\
\hline 941212 & 1431 & 1.72 & 0.093 & 10.7 & 54 & 63 & 0.51 & 1.34 & 6.13 & 29 & 0.47 \\
\hline 941212 & 1655 & 1.78 & 0.093 & 10.7 & 56 & 64 & 0.50 & 1.14 & 6.07 & 28 & 0.21 \\
\hline 941212 & 1957 & 2.02 & 0.093 & 10.7 & 50 & 62 & 0.53 & 1.05 & 5.57 & 31 & 0.47 \\
\hline 941212 & 2256 & 2.28 & 0.093 & 10.7 & 58 & 63 & 0.47 & 1.03 & 6.78 & 26 & 0.23 \\
\hline 941213 & 0156 & 2.56 & 0.093 & 10.7 & 64 & 63 & 0.44 & 1.05 & 7.54 & 23 & -0.03 \\
\hline 941213 & 0456 & 2.64 & 0.083 & 12.0 & 56 & 62 & 0.47 & 1.74 & 7.61 & 22 & 0.37 \\
\hline 941213 & 1056 & 2.91 & 0.083 & 12.0 & 58 & 65 & 0.45 & 1.05 & 7.25 & 24 & 0.16 \\
\hline 941213 & 1355 & 2.97 & 0.083 & 12.0 & 56 & 64 & 0.45 & 1.30 & 7.74 & 23 & 0.30 \\
\hline 941213 & 1656 & 3.01 & 0.074 & 13.6 & 64 & 65 & 0.46 & 1.23 & 6.82 & 25 & 0.12 \\
\hline 941213 & 1956 & 3.18 & 0.074 & 13.6 & 58 & 68 & 0.50 & 1.06 & 5.71 & 29 & 0.40 \\
\hline 941213 & 2256 & 3.63 & 0.074 & 13.6 & 60 & 66 & 0.44 & 0.87 & 7.08 & 23 & 0.08 \\
\hline 941214 & 0156 & 3.65 & 0.074 & 13.6 & 58 & 66 & 0.44 & 0.76 & 6.87 & 26 & 0.06 \\
\hline 941214 & 0456 & 3.04 & .074 & 13.6 & 76 & 66 & 0.48 & 0.79 & 6.06 & 29 & 0.00 \\
\hline 941214 & 0756 & 2.99 & 0.064 & 15.6 & 74 & 69 & 0.48 & 0.51 & 5.97 & 29 & -0.19 \\
\hline 941214 & 1056 & 3.08 & .074 & 13.6 & 62 & 68 & 0.46 & 0.57 & 6.00 & 28 & 0.22 \\
\hline 941214 & 1739 & 2.63 & 0.074 & 13.6 & 78 & 76 & 0.49 & 0.53 & 6.17 & 25 & -0.17 \\
\hline 941214 & 1953 & 2.60 & 0.074 & 13.6 & 76 & 78 & 0.50 & 0.74 & 6.30 & 25 & 0.11 \\
\hline 941214 & 2253 & 2.46 & 0.074 & 13.6 & 72 & 76 & 0.46 & 0.86 & 7.38 & 22 & 0.17 \\
\hline 941215 & 0153 & 2.29 & 0.074 & 13.6 & 72 & 75 & 0.47 & 0.64 & 7.30 & 22 & 0.03 \\
\hline 941215 & 0451 & 2.05 & 0.074 & 13.6 & 72 & 76 & 0.53 & 0.81 & 6.19 & 25 & 0.13 \\
\hline 941215 & 0751 & 2.17 & 0.054 & 18.5 & 68 & 73 & 0.46 & 0.80 & 7.43 & 21 & 0.34 \\
\hline 941215 & 1108 & 2.36 & 0.054 & 18.5 & 70 & 70 & 0.47 & 0.72 & 6.69 & 21 & 0.06 \\
\hline 941215 & 1422 & 2.52 & 0.054 & 18.5 & 70 & 67 & 0.50 & 0.28 & 5.55 & 26 & -0.26 \\
\hline 941215 & 1655 & 2.53 & 0.054 & 18.5 & 70 & 70 & 0.48 & 0.07 & 5.70 & 22 & 0.08 \\
\hline 941215 & 1955 & 2.49 & 0.054 & 18.5 & 70 & 69 & 0.49 & 0.11 & 5.07 & 27 & -0.11 \\
\hline 941215 & 2255 & 2.58 & 0.064 & 15.6 & 66 & 69 & 0.48 & 0.14 & 5.15 & 26 & 0.24 \\
\hline 941216 & 0155 & 2.59 & 0.064 & 15.6 & 70 & 69 & 0.46 & 0.12 & 5.97 & 23 & -0.13 \\
\hline 941216 & 0455 & 2.36 & 0.064 & 15 & 66 & 66 & 0.48 & 0.52 & & 29 & -0.06 \\
\hline 941216 & 0755 & 2.50 & 0.064 & 15.6 & 64 & 67 & 0.47 & 0.40 & 6.19 & 24 & 0.23 \\
\hline 941216 & 1113 & & 0.064 & 15.6 & 68 & 69 & 0.46 & 0.37 & 5.79 & 27 & 0.06 \\
\hline 941216 & 1403 & 2.78 & 0.064 & 15.6 & 66 & 68 & 0.44 & 0.13 & 6.10 & 22 & 0.16 \\
\hline 941216 & 1700 & 2.57 & 0.064 & 15.6 & 66 & 67 & 0.45 & 0.13 & 5.79 & 24 & 0.05 \\
\hline 941216 & 1955 & 2.25 & 0.064 & 15.6 & 70 & 67 & 0.49 & 0.35 & 5.53 & 26 & -0.10 \\
\hline 941217 & 0215 & 2.97 & 0.054 & 18.5 & 68 & 68 & 0.44 & 0.30 & 7.23 & 20 & 0.02 \\
\hline & 0455 & 3.00 & 0.054 & 18.5 & 66 & 66 & 0.37 & 0.37 & 9.60 & 14 & -0.03 \\
\hline 941217 & 0753 & 2.94 & 0.064 & 15.6 & 66 & 65 & 0.40 & 0.72 & 9.91 & 14 & -0.02 \\
\hline 941217 & 1052 & 3.20 & 0.064 & 15.6 & 64 & 65 & 0.41 & 0.92 & 9. & 18 & 0.07 \\
\hline 941217 & 1415 & 3.07 & 0.064 & 15.6 & 66 & 66 & 0.40 & 0.39 & 8.30 & 18 & -0.01 \\
\hline 941217 & 1655 & & 0.064 & 15.6 & 68 & 64 & 0.42 & 0.46 & 7.41 & 21 & -0.30 \\
\hline 941217 & 1955 & 2.78 & 0.064 & 15.6 & 66 & 64 & 0.41 & 0.59 & 7.88 & 20 & -0.20 \\
\hline 941217 & 2255 & 2.61 & 0.064 & 15.6 & 68 & 67 & 0.45 & 0.42 & 6.26 & 24 & -0.09 \\
\hline 941218 & 0155 & 2.4 & 4 & & 66 & 68 & 0.4 & 0. & 5.72 & 26 & 0.09 \\
\hline & 0455 & & 0.074 & & & 68 & 0.44 & 0.61 & 7. & 23 & 0.24 \\
\hline 941218 & 0755 & 2.22 & 0.074 & 13.6 & 66 & 66 & 0.44 & 0.73 & 7.44 & 22 & -0.01 \\
\hline & 1055 & & 0.074 & 13.6 & 68 & 71 & 0.48 & 0.74 & 6.38 & 27 & 0.11 \\
\hline 941218 & 1352 & 2.21 & 0.074 & 13.6 & 76 & 72 & 0.46 & 0.59 & 6. & 26 & -0.09 \\
\hline 941218 & 1655 & 1.94 & 0.074 & 13.6 & 64 & 69 & 0.52 & 0.49 & 5.03 & 34 & 0.19 \\
\hline 941218 & 1955 & 2.10 & 0.074 & 13.6 & 74 & 69 & 0.51 & 0.37 & 4.70 & 35 & -0.14 \\
\hline 941218 & 2255 & 2.59 & 0.074 & 13.6 & 70 & 69 & 0.45 & 0.61 & 6.70 & 23 & -0.02 \\
\hline 941219 & 0155 & 4.29 & 0.064 & 15.6 & 72 & 71 & 0.37 & 0.04 & 8.27 & 96 & -0.10 \\
\hline
\end{tabular}




\begin{tabular}{|c|c|c|c|c|c|c|c|c|c|c|c|}
\hline Date & $\begin{array}{l}\text { Time } \\
\text { GMT }\end{array}$ & $\begin{array}{l}H_{m 0} \\
\mathrm{~m}\end{array}$ & $\begin{array}{l}f_{p} \\
\mathrm{~Hz}\end{array}$ & $\begin{array}{l}T_{p} \\
\sec \end{array}$ & $\begin{array}{l}\theta_{p} \\
\text { deg }\end{array}$ & $\begin{array}{l}\theta_{0} \\
\text { deg }\end{array}$ & $\sigma$ & V & $\delta$ & $\begin{array}{l}\Delta \theta \\
\operatorname{deg}\end{array}$ & A \\
\hline 941219 & 0455 & 4.57 & 0.064 & 15.6 & 78 & 73 & 0.41 & 0.06 & 7.35 & 23 & -0.31 \\
\hline 941219 & 1652 & 4.33 & .064 & 15.6 & 78 & 75 & 0.39 & -0.06 & 8.35 & 17 & -0.20 \\
\hline 941219 & 1957 & 3.78 & 0.064 & 15.6 & 76 & 74 & 0.42 & 0.07 & 7.20 & 20 & -0.10 \\
\hline 941219 & 2258 & 3.48 & 0.064 & 15.6 & 78 & 75 & 0.40 & 0.07 & 7.80 & 20 & -0.29 \\
\hline 941220 & 0158 & 3.27 & 0.064 & 15.6 & 74 & 73 & 0.42 & 0.46 & 7.26 & 21 & -0.10 \\
\hline 941220 & 0458 & 3.11 & 0.064 & 15.6 & 72 & 73 & 0.41 & 0.43 & 7.81 & 22 & 0.06 \\
\hline 941220 & 0758 & 2.98 & 0.064 & 15.6 & 72 & 72 & 0.44 & 0.74 & 7.11 & 23 & 0.02 \\
\hline 941220 & 1058 & 3.01 & 0.074 & 13.6 & 58 & 69 & 0.46 & 0.90 & 7.03 & 27 & 0.16 \\
\hline 941220 & 1355 & 3.17 & 0.074 & 13.6 & 72 & 69 & 0.42 & 0.74 & 7.65 & 23 & -0.21 \\
\hline 941220 & 1656 & 3.25 & 0.064 & 15.6 & 72 & 71 & 0.40 & 0.59 & 8.07 & 21 & -0.12 \\
\hline 941220 & 1955 & 3.36 & 0.064 & 15.6 & 76 & 73 & 0.41 & 0.44 & 7.15 & 24 & -0.21 \\
\hline 941220 & 2255 & 3.13 & 0.074 & 13.6 & 76 & 74 & 0.44 & 0.48 & 6.76 & 25 & -0.17 \\
\hline 941221 & 0157 & 3.46 & 0.074 & 13.6 & 76 & 75 & 0.41 & 0.53 & 7.74 & 22 & -0.09 \\
\hline 941221 & 0451 & 3.86 & 0.064 & 15.6 & 74 & 76 & 0.39 & 0.43 & 7.98 & 19 & 0.23 \\
\hline 941221 & 0818 & 3.92 & 0.064 & 15.6 & 74 & 77 & 0.40 & 0.40 & 8.12 & 19 & 0.38 \\
\hline 941222 & 0157 & 4.00 & 0.064 & 15.6 & 76 & 79 & 0.41 & 0.39 & 7.50 & 21 & 0.25 \\
\hline 941222 & 0458 & 3.74 & 0.064 & 15.6 & 78 & 80 & 0.44 & 0.06 & 6.05 & 28 & 0.03 \\
\hline 941222 & 0757 & 3.66 & 0.064 & 15.6 & 74 & 78 & 0.46 & 0.06 & 5.90 & 26 & 0.17 \\
\hline 941222 & 1058 & 3.67 & 0.064 & 15.6 & 70 & 76 & 0.44 & 0.36 & 6.37 & 25 & 0.40 \\
\hline 941222 & 1357 & 3.71 & 0.064 & 15.6 & 70 & 76 & 0.40 & 0.55 & 7.21 & 22 & 0.55 \\
\hline 941222 & 1750 & 3.57 & 0.064 & 15.6 & 74 & 75 & 0.41 & 0.34 & 7.28 & 20 & 0.10 \\
\hline 941222 & 1958 & 3.44 & 0.064 & 15.6 & 70 & 73 & 0.43 & 0.57 & 7.00 & 20 & 0.26 \\
\hline 941222 & 2258 & 3.90 & 0.064 & 15.6 & 70 & 73 & 0.41 & 0.59 & 7.57 & 19 & 0.18 \\
\hline 941223 & 0157 & 3.87 & 0.064 & 15.6 & 68 & 75 & 0.44 & 0.44 & 6.33 & 27 & 0.30 \\
\hline 941223 & 0457 & 3.63 & 0.064 & 15.6 & 66 & 72 & 0.44 & 0.49 & 6.08 & 27 & 0.47 \\
\hline 941223 & 0758 & 3.47 & 0.064 & 15.6 & 66 & 71 & 0.45 & 0.53 & 6.21 & 25 & 0.31 \\
\hline 941223 & 1051 & 3.62 & 0.064 & 15.6 & 64 & 69 & 0.43 & 0.84 & 7.61 & 21 & 0.38 \\
\hline 941223 & 1351 & 3.85 & 0.064 & 15.6 & 64 & 69 & 0.41 & 0.86 & 7.91 & 21 & 0.25 \\
\hline 941223 & 1657 & 3.72 & 0.064 & 15.6 & 66 & 69 & 0.41 & 1.01 & 8.53 & 17 & 0.21 \\
\hline 941223 & 1951 & 3.44 & 0.064 & 15.6 & 68 & 72 & 0.42 & 0.91 & 8.05 & 22 & 0.25 \\
\hline 941223 & 2258 & 3.19 & 0.064 & 15.6 & 72 & 74 & 0.45 & 1.30 & 8.29 & 19 & 0.05 \\
\hline 941224 & 0157 & 2.82 & 0.064 & 15.6 & 70 & 76 & 0.48 & 1.19 & 6.97 & 25 & 0.40 \\
\hline 941224 & 0457 & 2.71 & 0.074 & 13.6 & 82 & 80 & & 0.70 & & 26 & -0.05 \\
\hline 941224 & 0757 & 2.49 & 0.074 & 13.6 & 72 & 80 & 0.50 & 0.94 & 6.03 & 29 & 0.17 \\
\hline 941224 & 1051 & 2.52 & 0.074 & 13.6 & 76 & 81 & & 1.47 & 7.91 & 20 & 0.23 \\
\hline 941224 & 1351 & 2.62 & 0.074 & 13.6 & 68 & 79 & 0.48 & 1.23 & 6.63 & 26 & 0.63 \\
\hline 941224 & 1658 & 2.41 & 0.074 & 13.6 & 68 & 80 & 0.51 & 0.93 & 5.74 & 31 & 0.44 \\
\hline 941224 & 1958 & 2.61 & 0.074 & 13.6 & 76 & 78 & 0.51 & 0.61 & 5.30 & 34 & 0.15 \\
\hline 941224 & 2251 & 3.10 & 0.074 & 13.6 & 80 & 72 & 0.51 & 0.20 & 4.19 & 37 & -0.06 \\
\hline 941225 & 0157 & 3.28 & 0.123 & 8.2 & 76 & 72 & 0.48 & 0.15 & 4.74 & 33 & -0.09 \\
\hline 941225 & 0457 & 3.42 & 0.093 & 10.7 & 80 & 74 & & 0.12 & 4. & 36 & -0.13 \\
\hline 941225 & 0757 & 4.15 & 0.093 & 10.7 & 82 & 75 & 0.44 & -0.21 & 4.42 & 32 & -0.29 \\
\hline 941225 & 1040 & 4.39 & 0.083 & 12.0 & 82 & 75 & 0.42 & -0.29 & 5.51 & 26 & -0.36 \\
\hline 941225 & 1351 & 4.48 & 0.093 & 10.7 & 84 & 74 & 0.45 & -0.18 & 4.37 & 33 & -0.35 \\
\hline 941225 & 1658 & 4.53 & 0.083 & 12.0 & 68 & 70 & 0.47 & 0.05 & 4.33 & 34 & -0.05 \\
\hline 941225 & 1957 & 4.16 & 0.083 & 12.0 & 80 & 69 & 0.47 & -0.03 & 4.32 & 35 & -0.18 \\
\hline 941225 & 2258 & 4.18 & 0.093 & 10.7 & 72 & 68 & 0.43 & 0.14 & 5.16 & 29 & -0.03 \\
\hline & 0158 & 4.25 & 0.074 & 13.6 & 72 & 69 & 0.44 & 0.11 & 4.88 & 30 & -0.07 \\
\hline 941226 & 0457 & 4.00 & 0.083 & 12.0 & 78 & 70 & 0.4 & 0.12 & 5.37 & 31 & -0.33 \\
\hline 941226 & 0757 & 3.57 & 0.093 & 10.7 & 64 & 68 & 0.46 & 0.17 & 4.69 & 34 & 0.01 \\
\hline 941226 & 1057 & 3.15 & 0.103 & 9.7 & 62 & 66 & 0.48 & 0.22 & 4.50 & 36 & 0.06 \\
\hline 941226 & 1359 & 2.88 & 0.103 & 9.7 & 54 & 66 & 0.51 & 0.34 & 3.69 & 43 & 0.44 \\
\hline 941226 & 1658 & 2.58 & 0.083 & 12.0 & 58 & 68 & 0.52 & 0.27 & 3.78 & 43 & 0.18 \\
\hline \multicolumn{12}{|c|}{ (Sheet 44 of 45 ) } \\
\hline
\end{tabular}




\begin{tabular}{|c|c|c|c|c|c|c|c|c|c|c|c|}
\hline Date & $\begin{array}{l}\text { Time } \\
\text { GMT }\end{array}$ & $\begin{array}{l}H_{m o} \\
\mathrm{~m}\end{array}$ & $\begin{array}{l}f_{p} \\
\mathrm{~Hz}\end{array}$ & $\begin{array}{l}T_{p} \\
\text { sec }\end{array}$ & $\begin{array}{l}\theta_{p} \\
\text { deg }\end{array}$ & $\begin{array}{l}\theta_{0} \\
\operatorname{deg}\end{array}$ & o & V & $\delta$ & $\begin{array}{l}\Delta \theta \\
\operatorname{deg}\end{array}$ & A \\
\hline $\begin{array}{l}941226 \\
941226\end{array}$ & $\begin{array}{l}1957 \\
2257\end{array}$ & $\begin{array}{l}2.63 \\
2.56\end{array}$ & $\begin{array}{l}0.083 \\
0.083\end{array}$ & \begin{tabular}{|l|}
12.0 \\
12.0
\end{tabular} & $\begin{array}{l}56 \\
60\end{array}$ & $\begin{array}{l}66 \\
64\end{array}$ & $\begin{array}{l}0.50 \\
0.48\end{array}$ & $\begin{array}{l}0.27 \\
0.37\end{array}$ & $\begin{array}{l}4.10 \\
4.86\end{array}$ & $\begin{array}{l}39 \\
33\end{array}$ & $\begin{array}{l}0.26 \\
0.15\end{array}$ \\
\hline 941227 & 0158 & 2.67 & 0.083 & 12.0 & 60 & 63 & 0.45 & 0.60 & 5.98 & 27 & 0.22 \\
\hline 941227 & 0458 & 2.71 & 0.074 & 13.6 & 64 & 66 & 0.46 & 0.54 & 6.08 & 28 & 0.16 \\
\hline 941227 & 0757 & 2.72 & 0.074 & 13.6 & 58 & 65 & 0.46 & 0.60 & 6.85 & 27 & 0.20 \\
\hline 941227 & 1058 & 2.98 & 0.074 & 13.6 & 70 & 67 & 0.41 & 0.35 & 7.40 & 23 & -0.04 \\
\hline 941227 & 1357 & 3.18 & 0.074 & 13.6 & 68 & 68 & 0.41 & 0.70 & 8.69 & 21 & 0.03 \\
\hline 941227 & 1657 & 3.63 & 0.074 & 13.6 & 74 & 72 & 0.39 & 0.12 & 8.29 & 21 & -0.20 \\
\hline 941227 & 1958 & 3.30 & 0.074 & 13.6 & 74 & 72 & 0.39 & 0.52 & 8.56 & 21 & -0.16 \\
\hline 941227 & 2257 & 3.28 & 0.074 & 13.6 & 78 & 75 & 0.40 & 0.24 & 7.63 & 22 & -0.18 \\
\hline 941228 & 0158 & 2.85 & 0.064 & 15.6 & 74 & 74 & 0.40 & 0.37 & 8.69 & 21 & 0.03 \\
\hline 941228 & 0458 & 3.02 & 0.074 & 13.6 & 74 & 74 & 0.40 & 0.53 & 8.73 & 21 & -0.02 \\
\hline 941228 & 0758 & 2.90 & 0.074 & 13.6 & 70 & 74 & 0.42 & 0.52 & 7.16 & 24 & 0.13 \\
\hline 941228 & 1047 & 2.74 & 0.074 & 13.6 & 74 & 73 & 0.40 & 0.51 & 7.83 & 22 & -0.06 \\
\hline 941228 & 1247 & 2.63 & 0.074 & 13.6 & 72 & 72 & 0.42 & 0.67 & 7.70 & 23 & 0.01 \\
\hline 941228 & 1455 & 2.58 & 0.083 & 12.0 & 76 & 71 & 0.45 & 0.34 & 5.86 & 28 & -0.03 \\
\hline 941228 & 1655 & 2.70 & 0.074 & 13.6 & 64 & 70 & 0.47 & 0.36 & 5.28 & 30 & 0.08 \\
\hline 941228 & 1848 & 2.75 & 0.083 & 12.0 & 66 & 70 & 0.48 & 0.40 & 5.30 & 30 & 0.14 \\
\hline 941228 & 2055 & 2.84 & 0.074 & 13.6 & 56 & 71 & 0.50 & 0.34 & 4.85 & 35 & 0.17 \\
\hline 941228 & 2255 & 2.94 & 0.083 & 12.0 & 76 & 69 & 0.48 & 0.11 & 4.73 & 34 & -0.02 \\
\hline 941229 & 0055 & 2.67 & 0.083 & 12.0 & 76 & 69 & 0.51 & 0.22 & 4.37 & 36 & -0.01 \\
\hline 941229 & 0255 & 2.65 & 0.083 & 12.0 & 76 & 71 & 0.47 & 0.38 & 5.31 & 32 & 0.00 \\
\hline 941229 & 0455 & 2.55 & 0.083 & 12.0 & 56 & 71 & 0.49 & 0.56 & 5.50 & 33 & 0.17 \\
\hline 941229 & 0655 & 2.56 & 0.093 & 10.7 & 56 & 70 & 0.48 & 0.55 & 5.56 & 32 & 0.18 \\
\hline 941229 & 0855 & 2.49 & 0.093 & 10.7 & 72 & 70 & 0.49 & 0.49 & 5.63 & 31 & 0.03 \\
\hline 941229 & 1055 & 2.39 & 0.083 & 12.0 & 60 & 69 & 0.48 & 0.66 & 6.03 & 31 & 0.16 \\
\hline 941229 & 1255 & 2.47 & 0.074 & 13.6 & 60 & 70 & 0.50 & 0.65 & 5.35 & 33 & 0.18 \\
\hline 941229 & 1510 & 2.46 & 0.093 & 10.7 & 56 & 70 & 0.54 & 0.61 & 4.62 & 39 & 0.33 \\
\hline 941229 & 1704 & 2.36 & 0.074 & 13.6 & 54 & 70 & 0.55 & 0.78 & 4.69 & 38 & 0.46 \\
\hline 941229 & 1855 & 2.34 & 0.083 & 12.0 & 54 & 70 & 0.54 & 0.68 & 4.27 & 40 & 0.27 \\
\hline 941229 & 2055 & 2.48 & 0.093 & 10.7 & 56 & 69 & 0.55 & 0.65 & 4.00 & 40 & 0.41 \\
\hline 941229 & 2255 & 2.44 & 0.093 & 10.7 & 52 & 66 & 0.53 & 0.95 & 5.07 & 36 & 0.37 \\
\hline 941230 & 0055 & 2.33 & 0.093 & 10.7 & 50 & 62 & 0.51 & 1.07 & 5.35 & 34 & 0.46 \\
\hline 941230 & 0255 & 2.16 & 0.093 & 10.7 & 56 & 65 & 0.57 & 1.12 & 4.62 & 38 & 0.33 \\
\hline 941230 & 0455 & 2.07 & 0.093 & 10.7 & 60 & 70 & 0.57 & 0.99 & 4.85 & 35 & 0.14 \\
\hline 941230 & 0655 & 2.01 & 0.103 & 9.7 & 72 & 75 & 0.59 & 0.69 & 4.18 & 40 & 0.23 \\
\hline 941230 & 0855 & 2.06 & 0.103 & 9.7 & 70 & 73 & 0.58 & 0.87 & 4.50 & 37 & 0.29 \\
\hline 941230 & 1056 & 2.07 & 0.054 & 18.5 & 70 & 72 & 0.57 & 1.10 & 4.82 & 33 & 0.10 \\
\hline 941230 & 1339 & 2.21 & 0.054 & 18.5 & 70 & 71 & 0.54 & 1.11 & 5.40 & 23 & 0.02 \\
\hline 941230 & 1440 & 2.30 & 0.054 & 18.5 & 70 & 70 & 0.52 & 1.18 & 5.87 & 21 & -0.19 \\
\hline 941230 & 1655 & 2.20 & 0.054 & 18.5 & 70 & 72 & 0.52 & 1.26 & 5.90 & 22 & 0.06 \\
\hline 941230 & 1855 & 2.26 & 0.054 & 18.5 & 68 & 73 & 0.49 & 1.46 & 6.61 & 18 & 0.29 \\
\hline 941230 & 2055 & 2.39 & 0.054 & 18.5 & 76 & 78 & 0.49 & 0.95 & 5.99 & 24 & 0.08 \\
\hline 941230 & 2255 & 2.44 & 0.054 & 18.5 & 80 & 83 & 0.51 & 0.59 & 5.18 & 29 & 0.11 \\
\hline 941231 & 0055 & 2.38 & 0.054 & 18.5 & 82 & 82 & 0.48 & 0.89 & 6.65 & 22 & -0.12 \\
\hline 941231 & 0255 & 2.35 & 0.064 & 15.6 & 78 & 85 & 0.4 & 0.6 & 6.24 & 25 & 0.39 \\
\hline 941231 & 0455 & 2.57 & 0.064 & 15.6 & 76 & 82 & 0.44 & 0.79 & 7.06 & 22 & 0.36 \\
\hline 941231 & 0655 & 2.47 & 0.064 & 15.6 & 74 & 81 & 0.46 & 1.30 & 7.53 & 17 & 0.55 \\
\hline 941231 & 1057 & 2.55 & 0.064 & 15.6 & 80 & 82 & 0.45 & 1.08 & 8.54 & 15 & 0.03 \\
\hline 941231 & 1358 & 2.48 & 0.064 & 15.6 & 76 & 84 & 0.44 & 1.02 & 7.28 & 22 & 0.45 \\
\hline 941231 & 1658 & 2.57 & 0.064 & 15.6 & 78 & 86 & 0.43 & 1.06 & 7.23 & 22 & 0.43 \\
\hline 941231 & 1955 & 2.87 & 0.064 & 15.6 & 82 & 86 & 0.41 & 0.71 & 7.19 & 20 & 0.27 \\
\hline 941231 & 2257 & 2.89 & 0.064 & 15.6 & 78 & 87 & 0.44 & 0.49 & 6.40 & 25 & 0.30 \\
\hline \multicolumn{12}{|c|}{ (Sheet 45 of 45 ) } \\
\hline
\end{tabular}





\section{Appendix B \\ Time Series Graphs of Bulk Parameters}




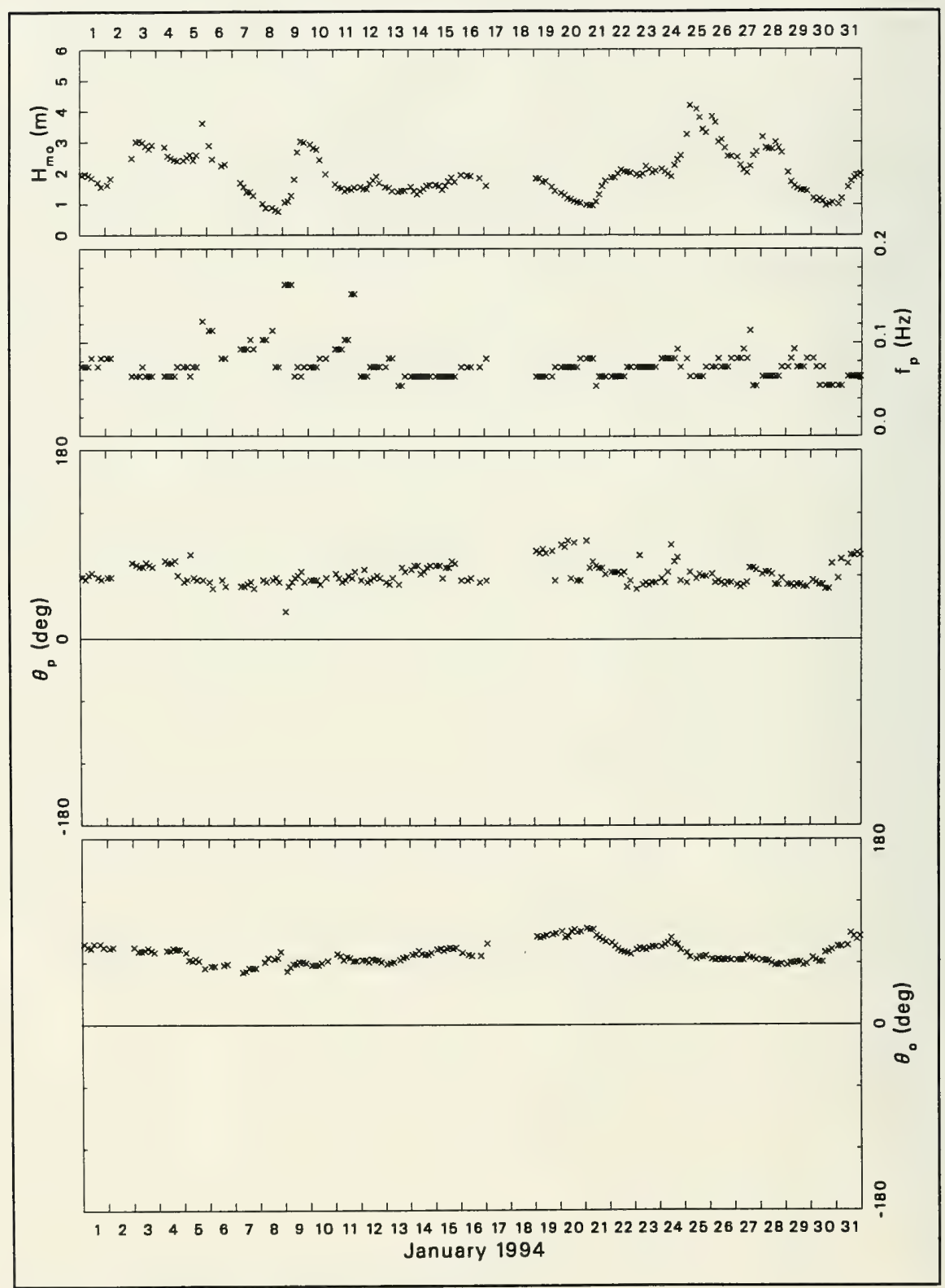

Figure B1. Bulk data for January 1994 (Continued) 


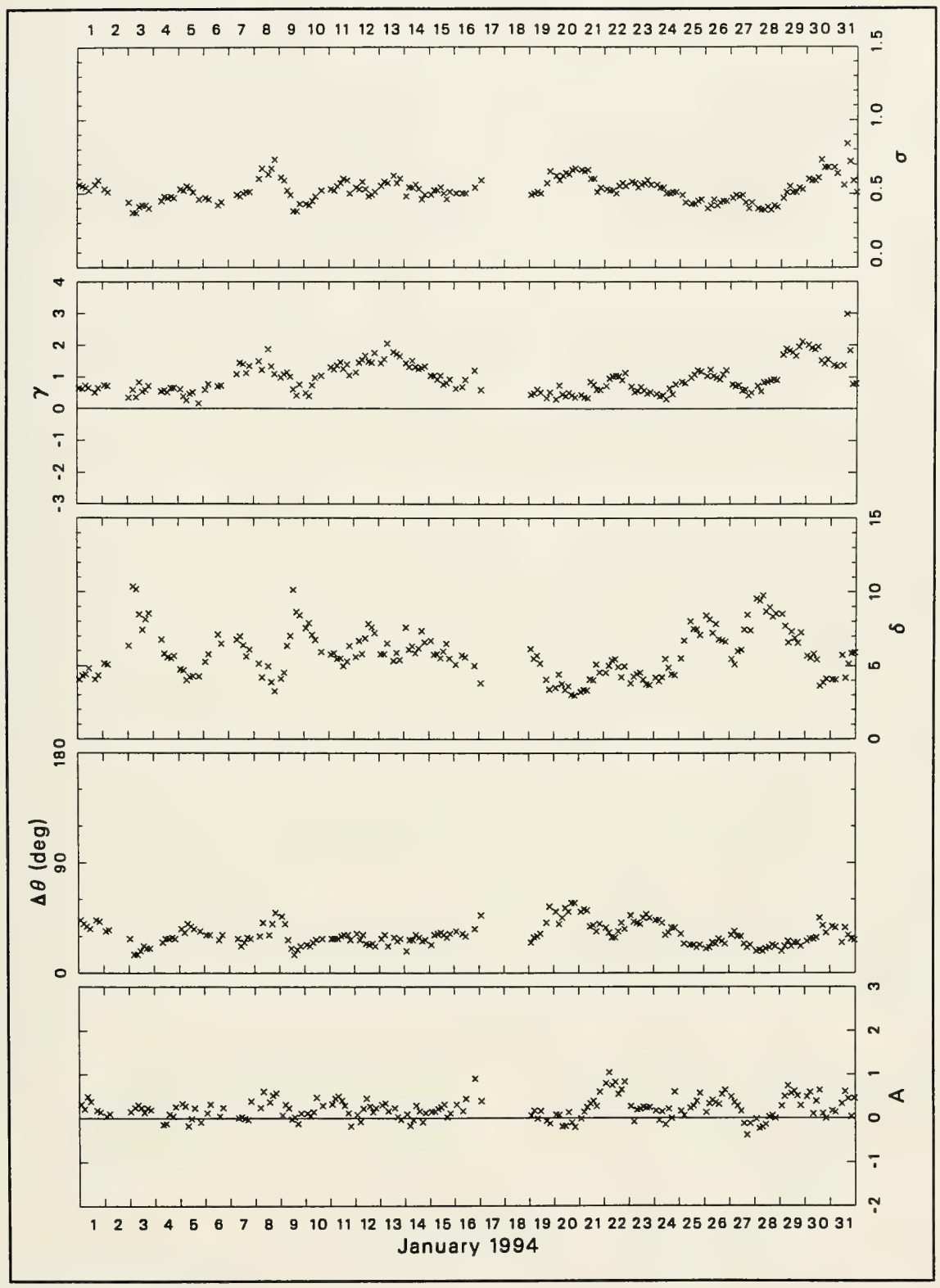

Figure B1. (Concluded) 


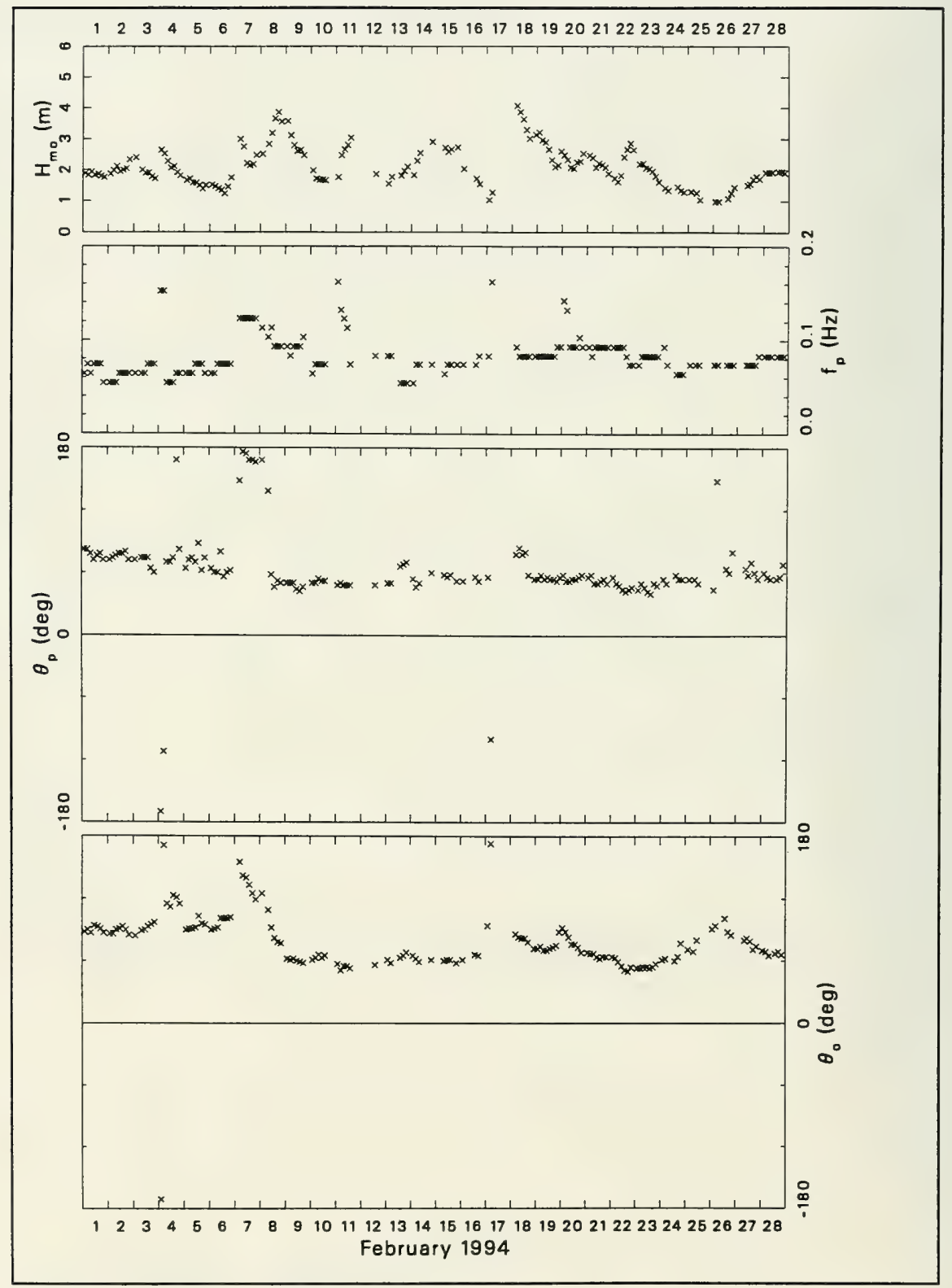

Figure B2. Bulk data for February 1994 (Continued) 


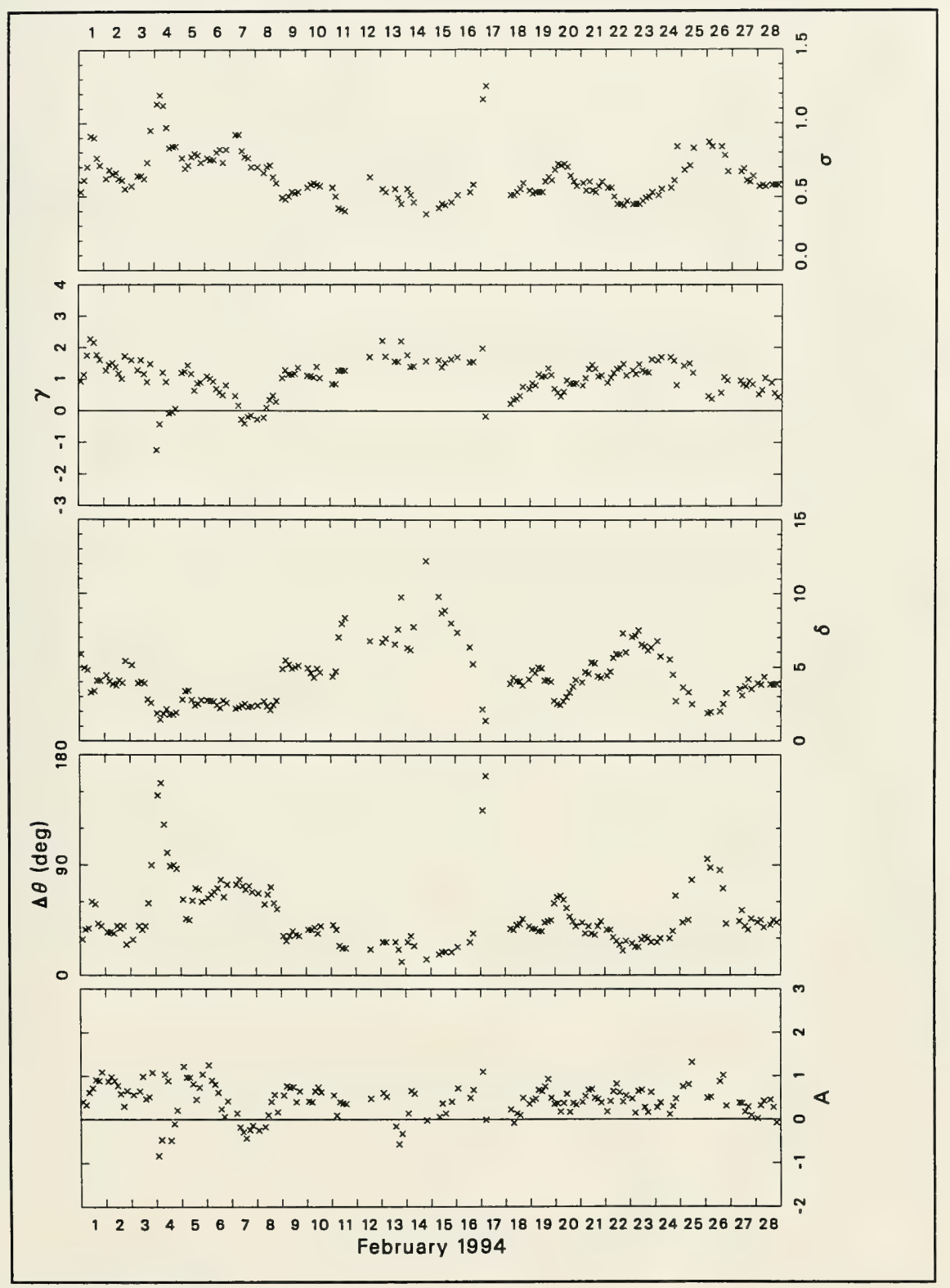

Figure B2. (Concluded) 


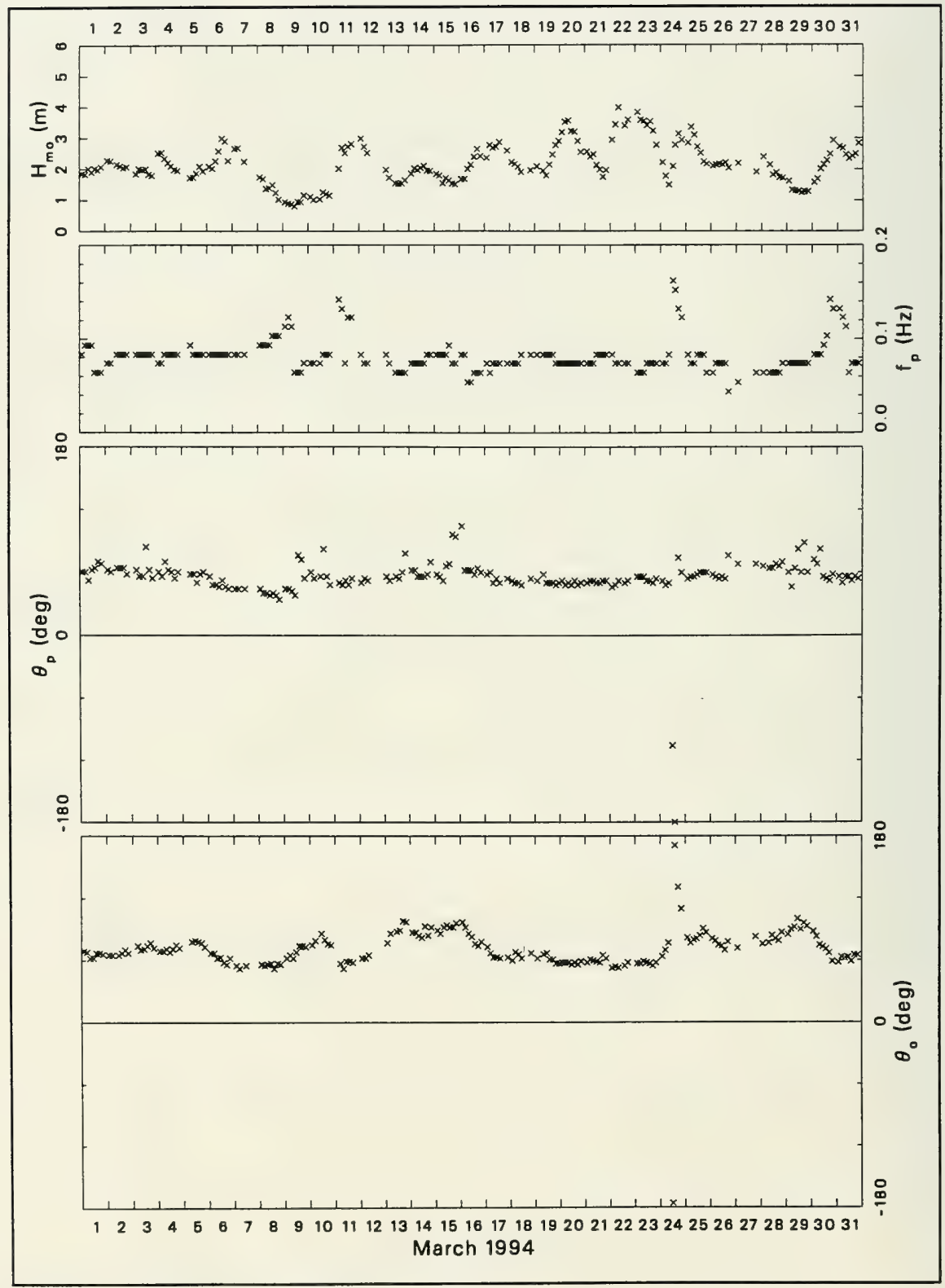

Figure B3. Bulk data for March 1994 (Continued) 


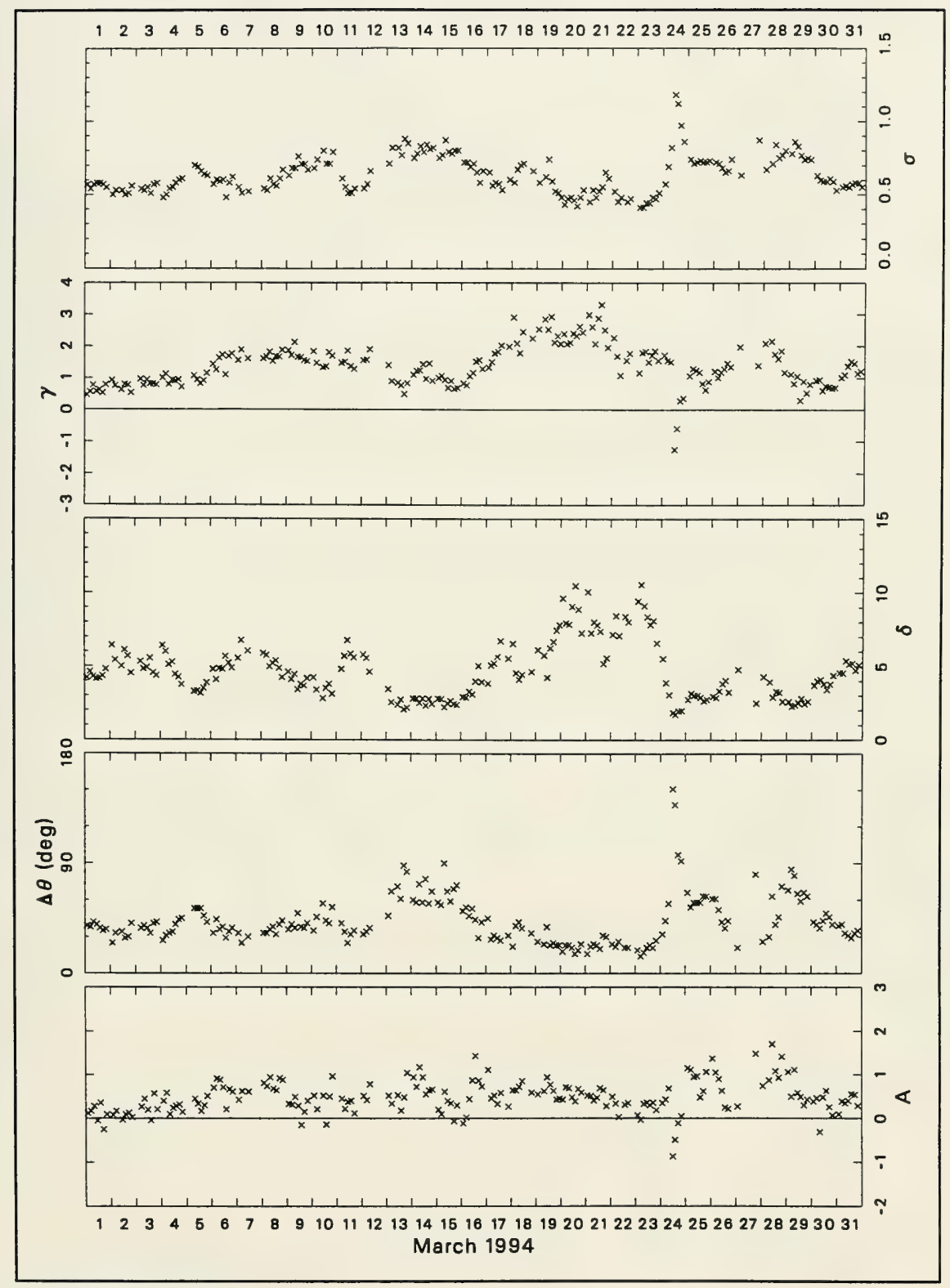

Figure B3. (Concluded) 


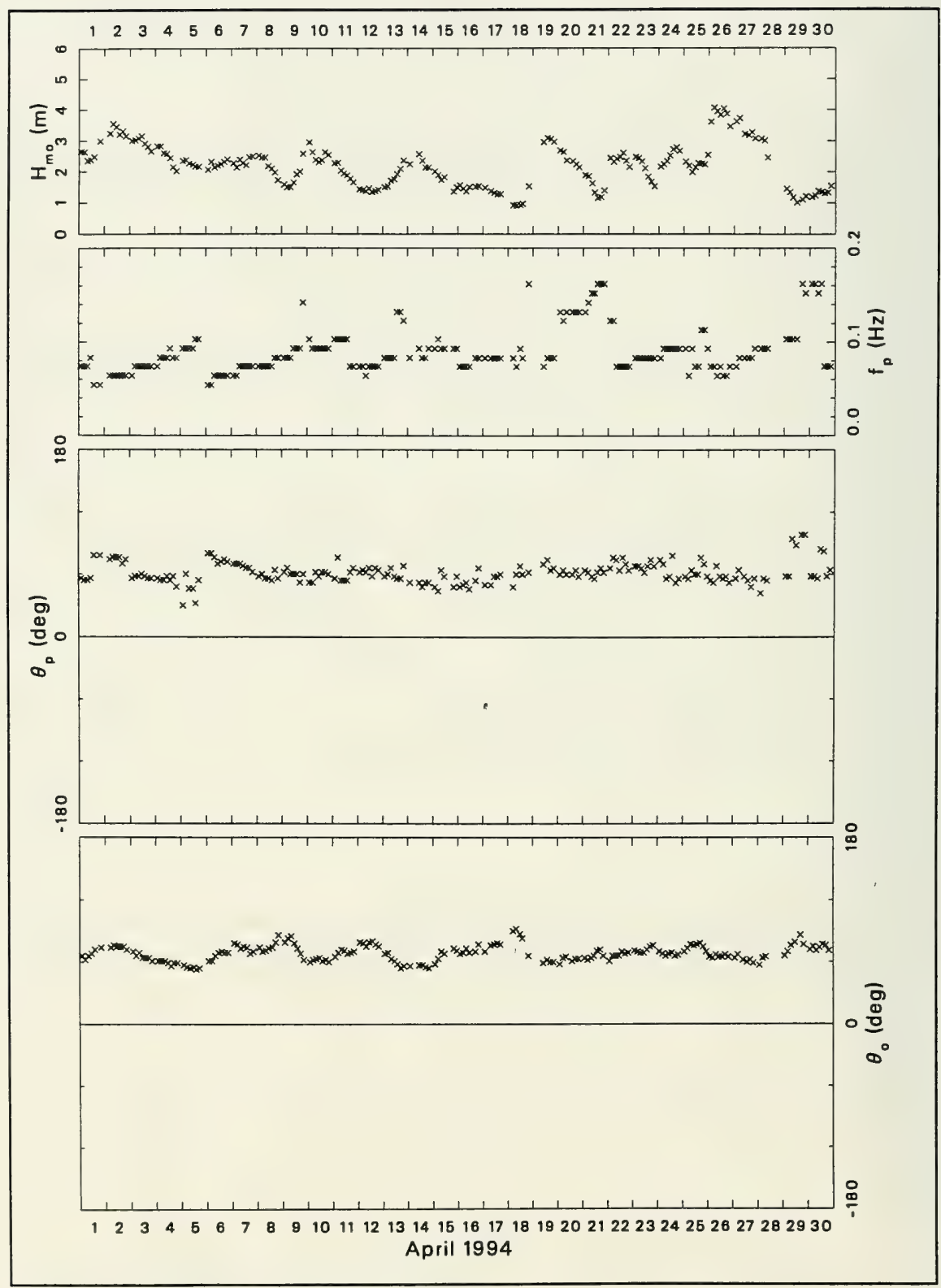

Figure B4. Bulk data for April 1994 (Continued) 


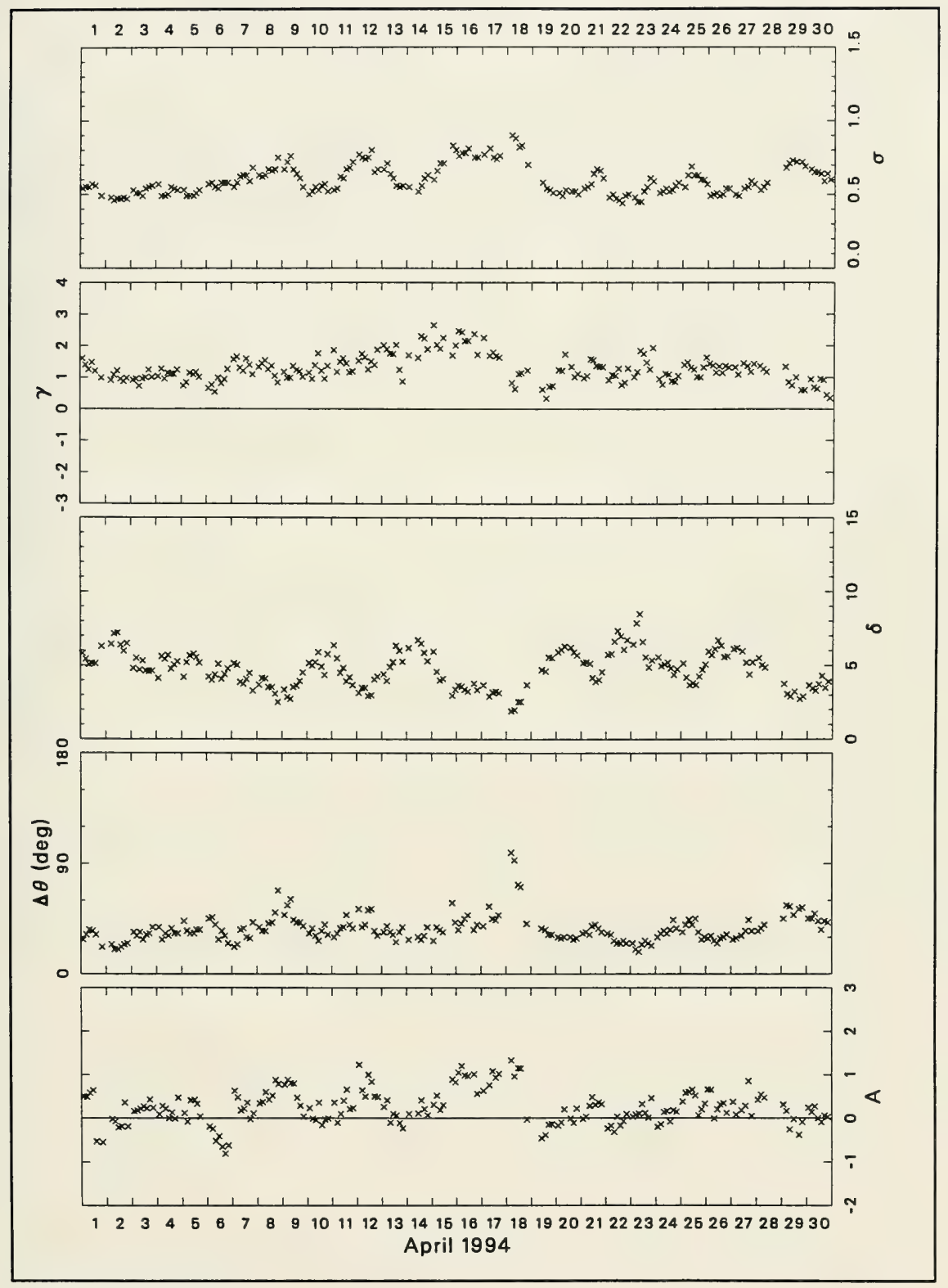

Figure B4. (Concluded) 


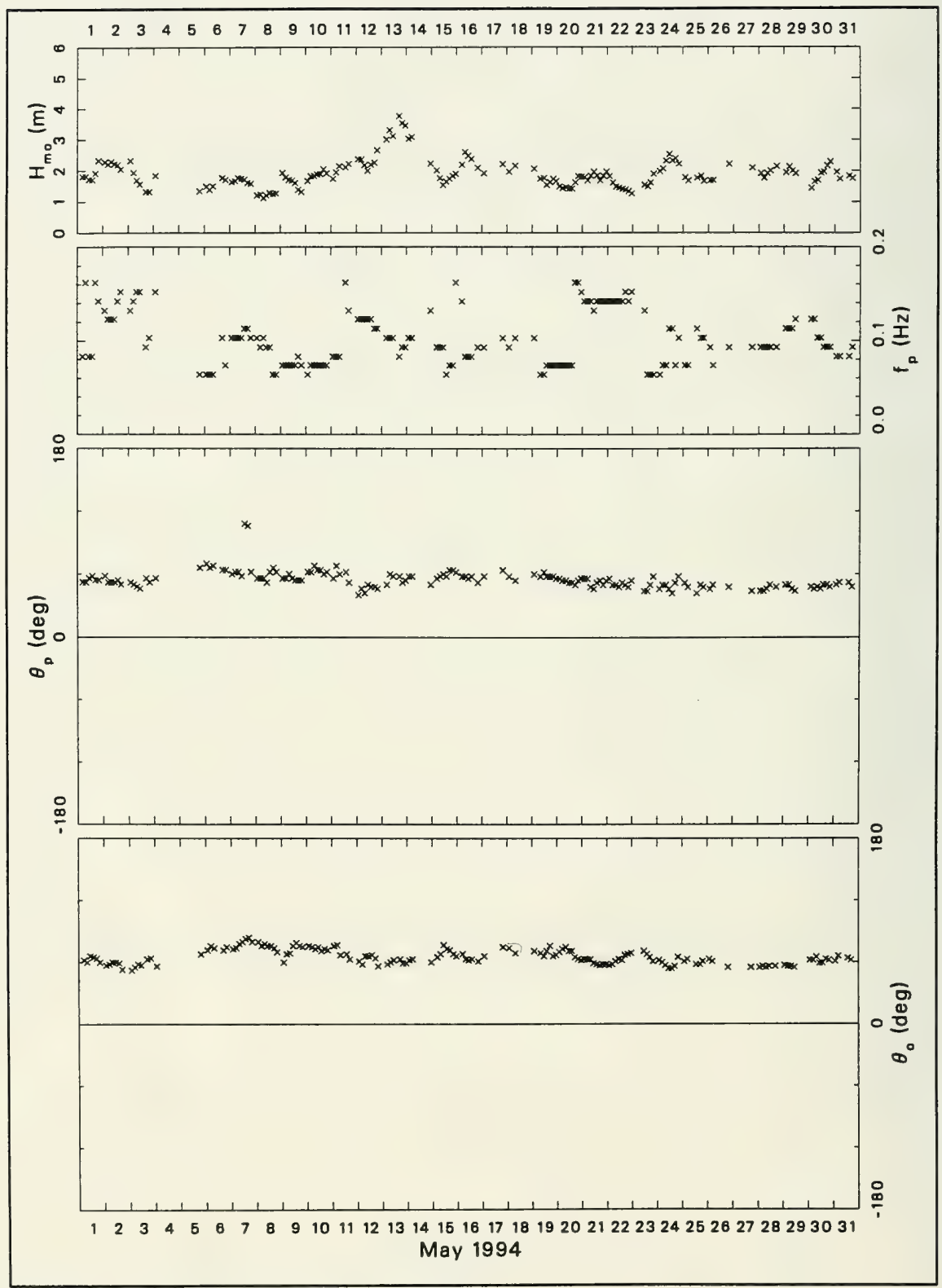

Figure B5. Bulk data for May 1994 (Continued) 


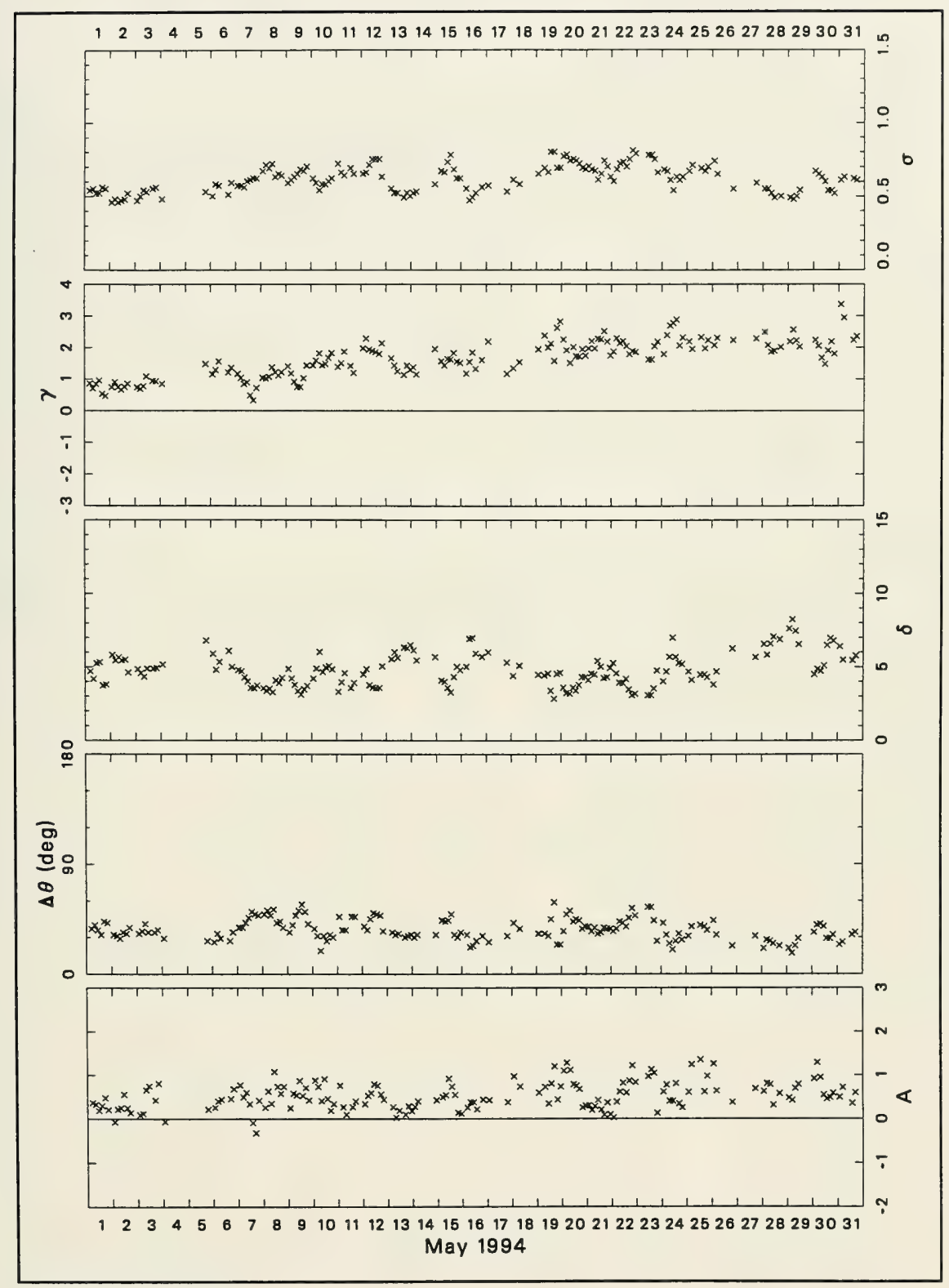

Figure B5. (Concluded) 


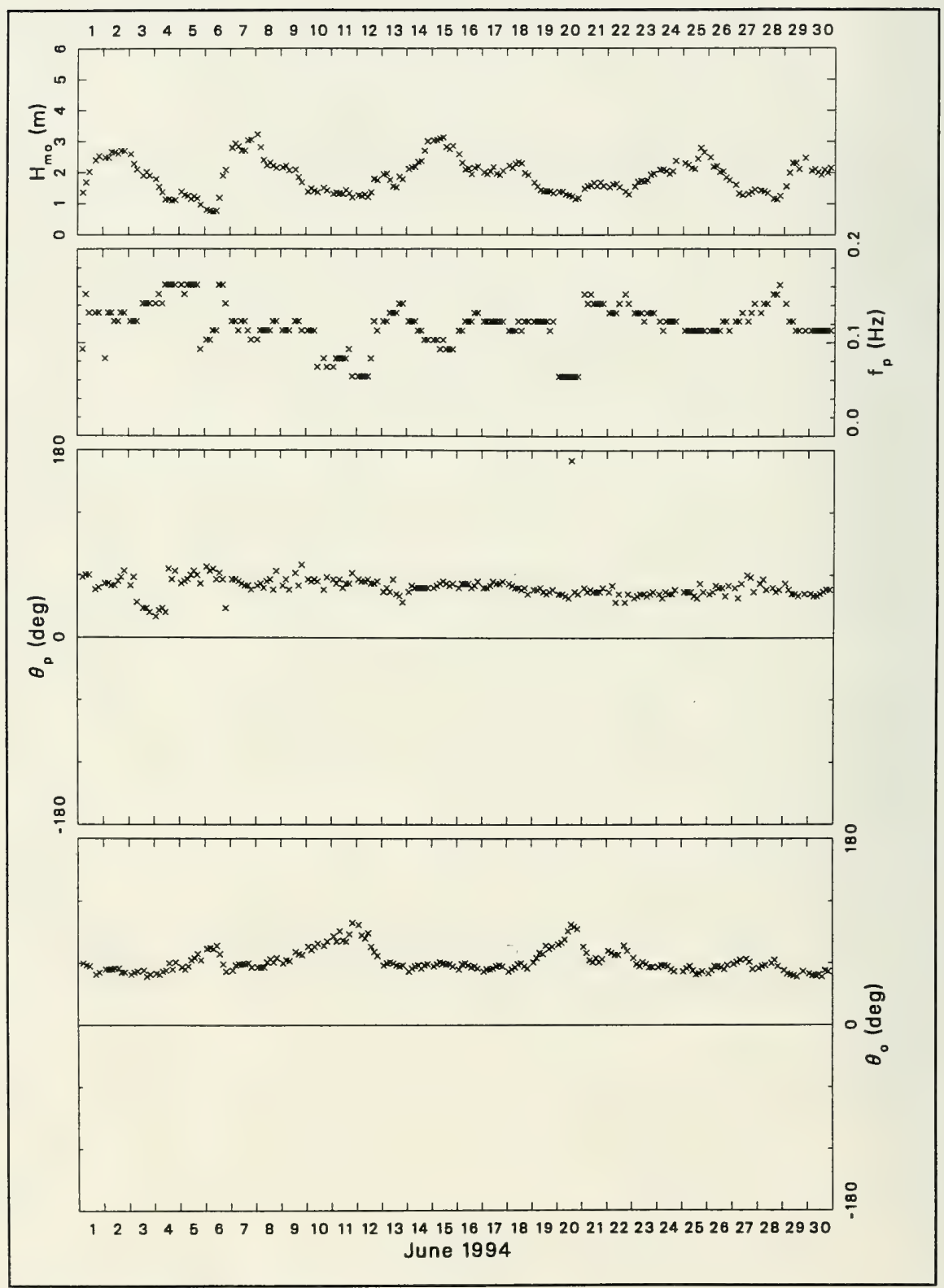

Figure B6. Bulk data for June 1994 (Continued) 


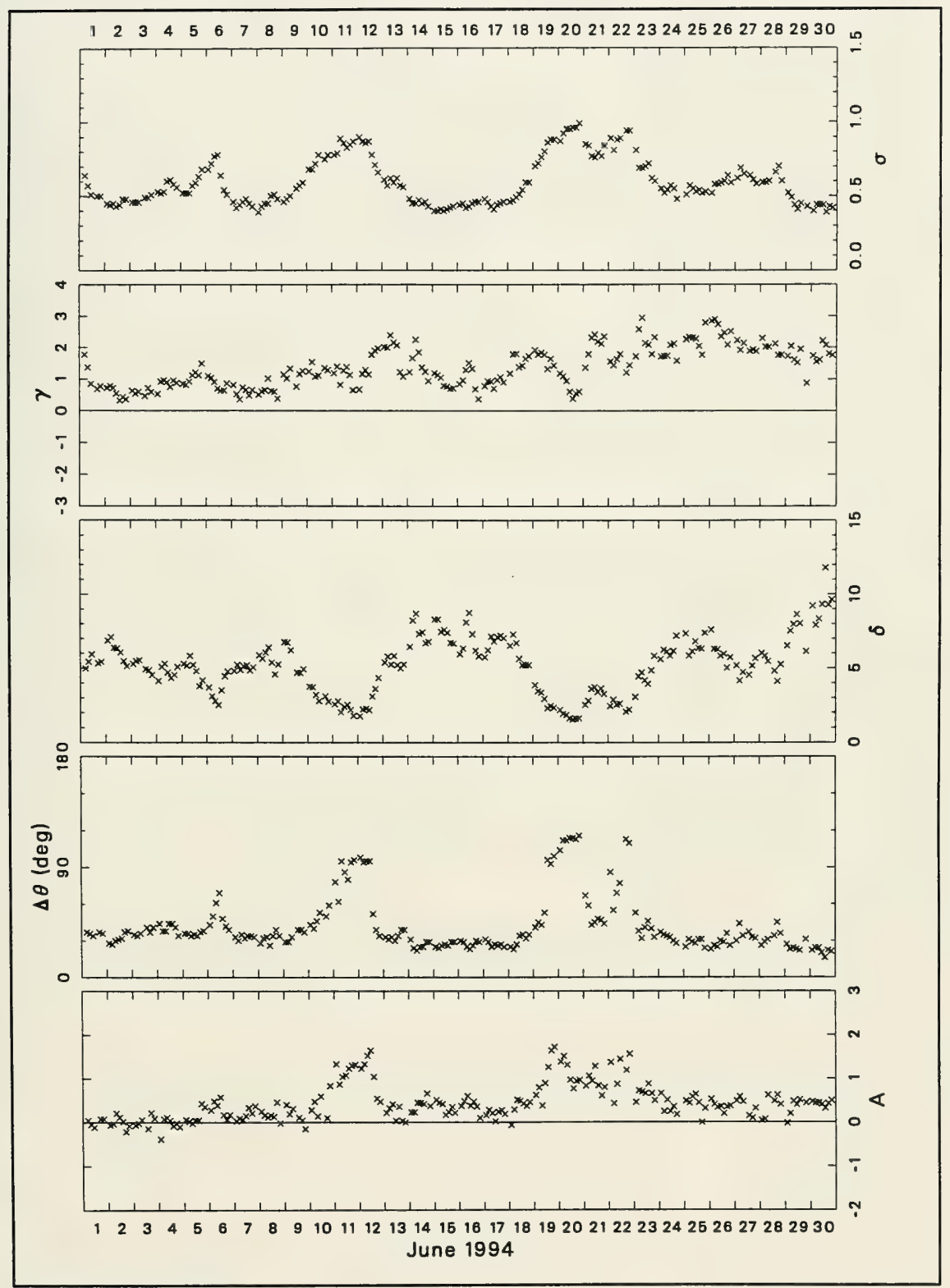

Figure B6. (Concluded) 


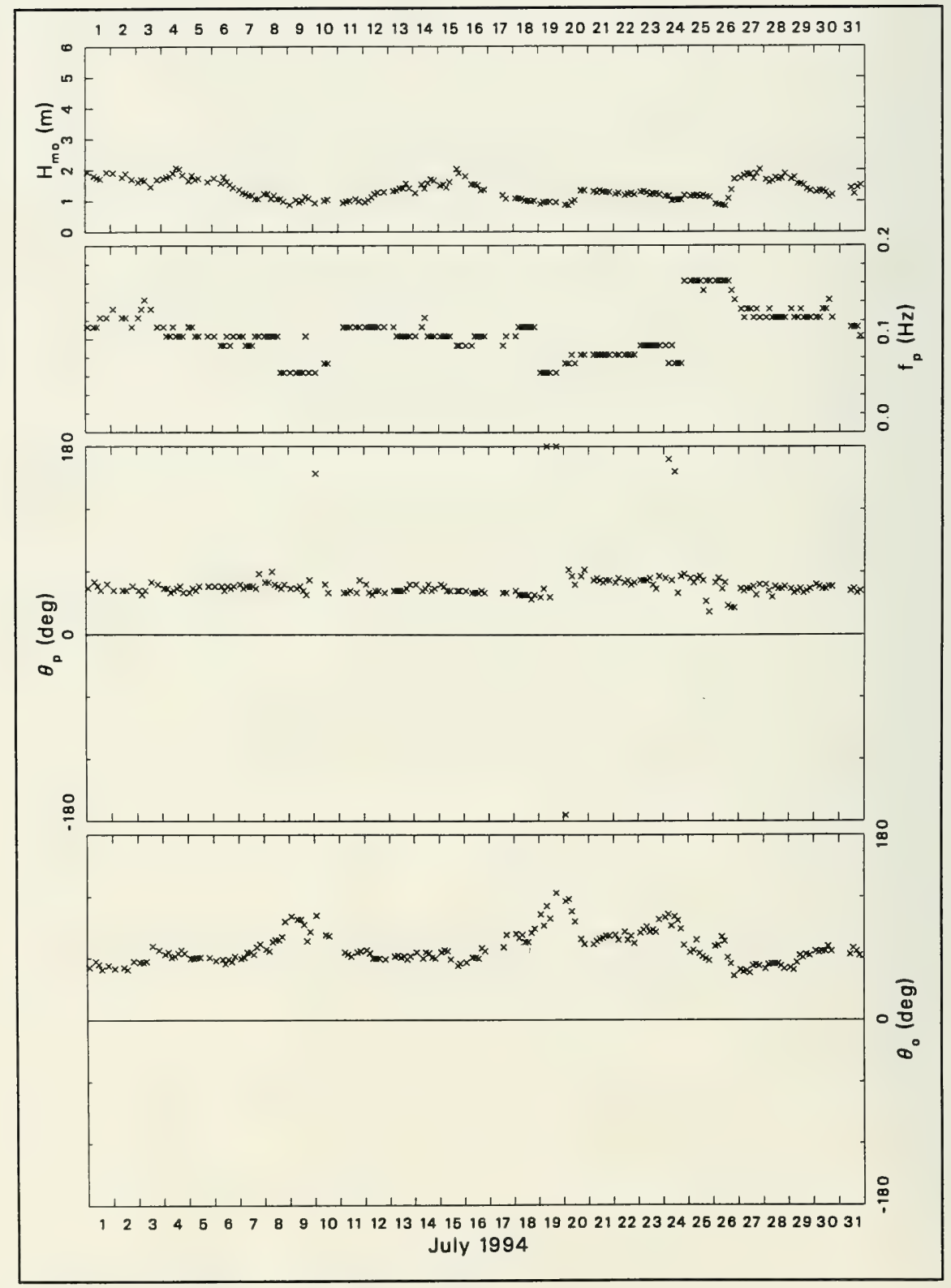

Figure B7. Bulk data for July 1994 (Continued) 

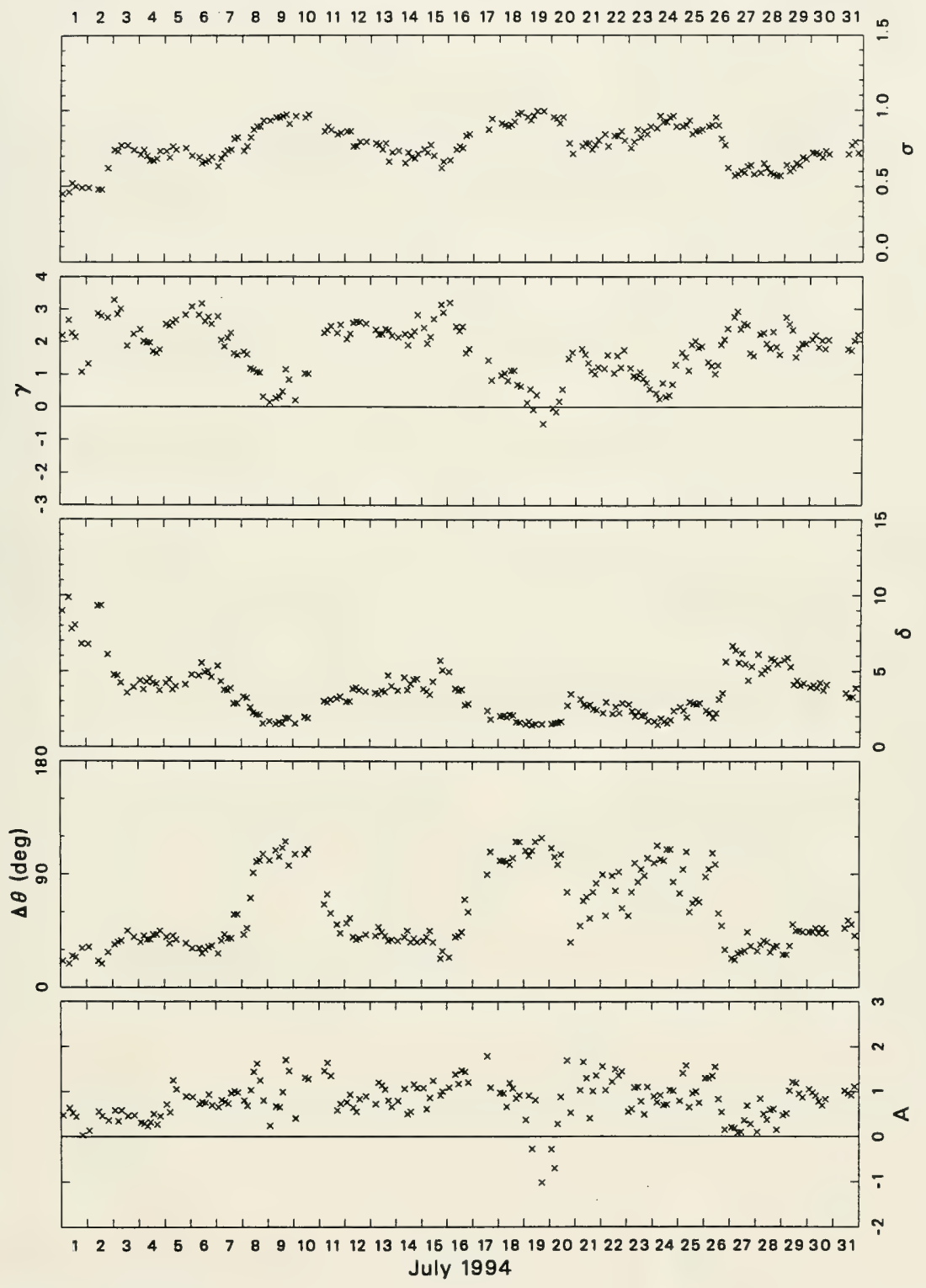

Figure B7. (Concluded)

Appendix B Time Series Graphs of Bulk Parameters

B15 


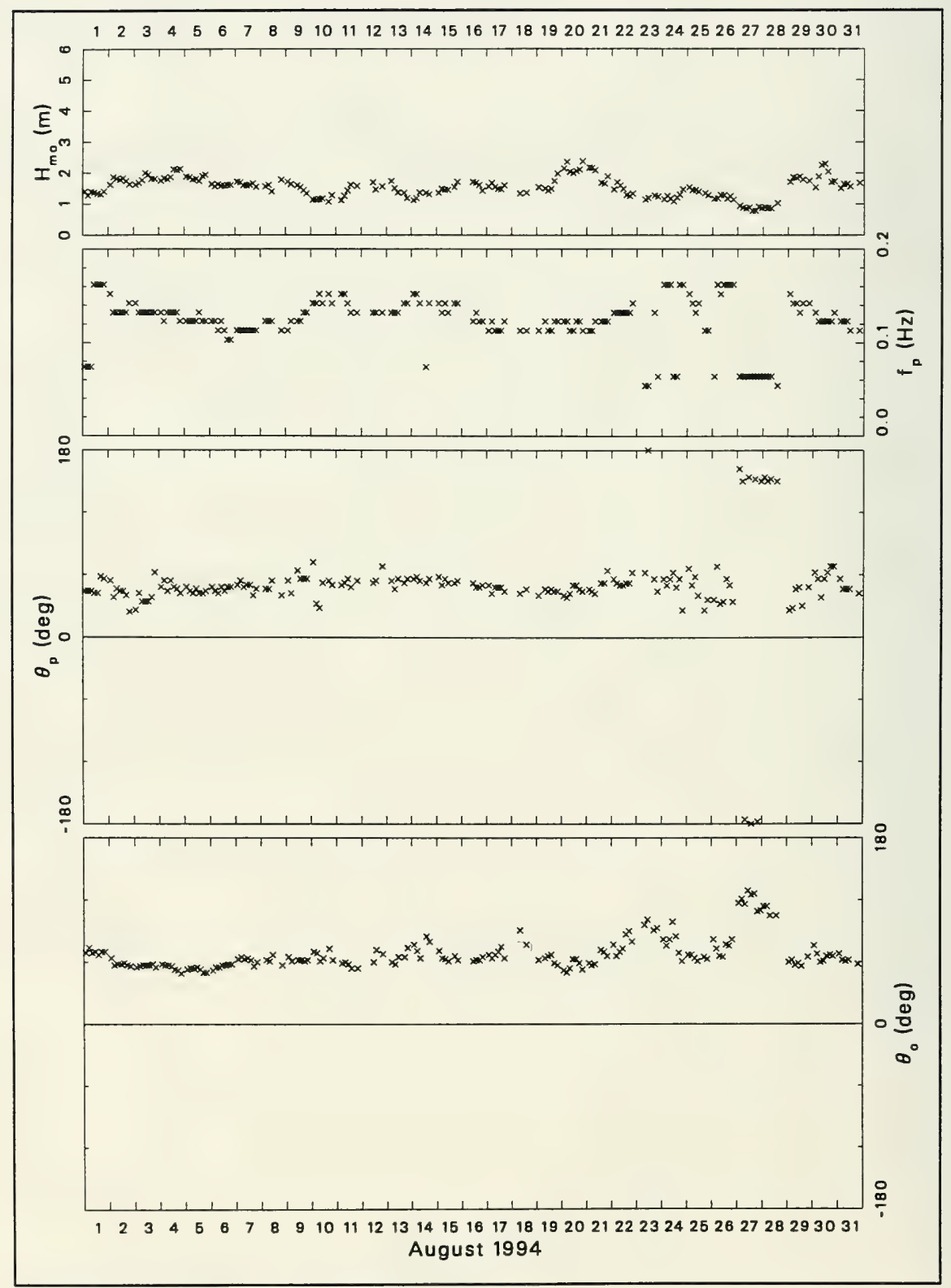

Figure B8. Bulk data for August 1994 (Continued) 


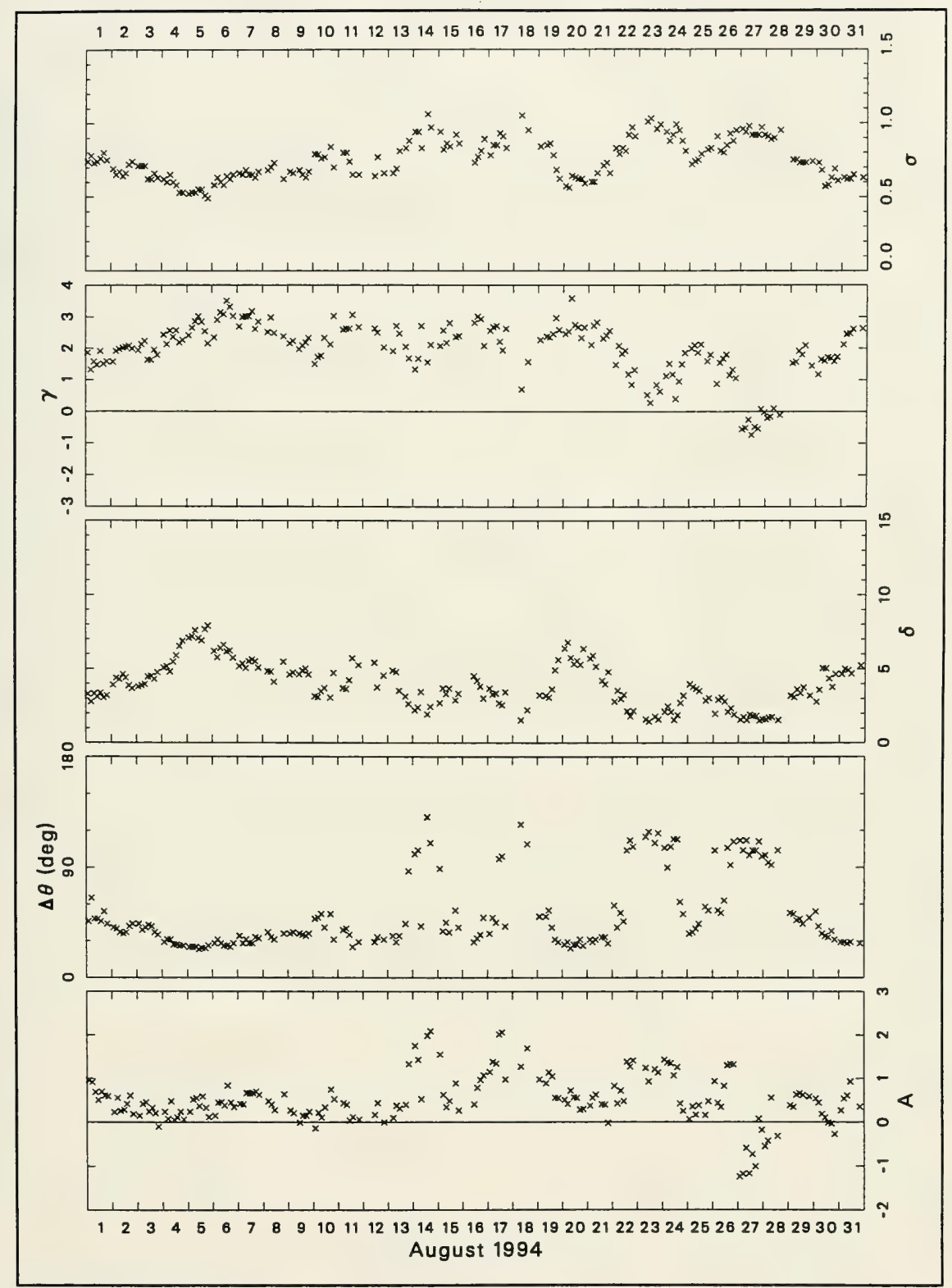

Figure B8. (Concluded) 


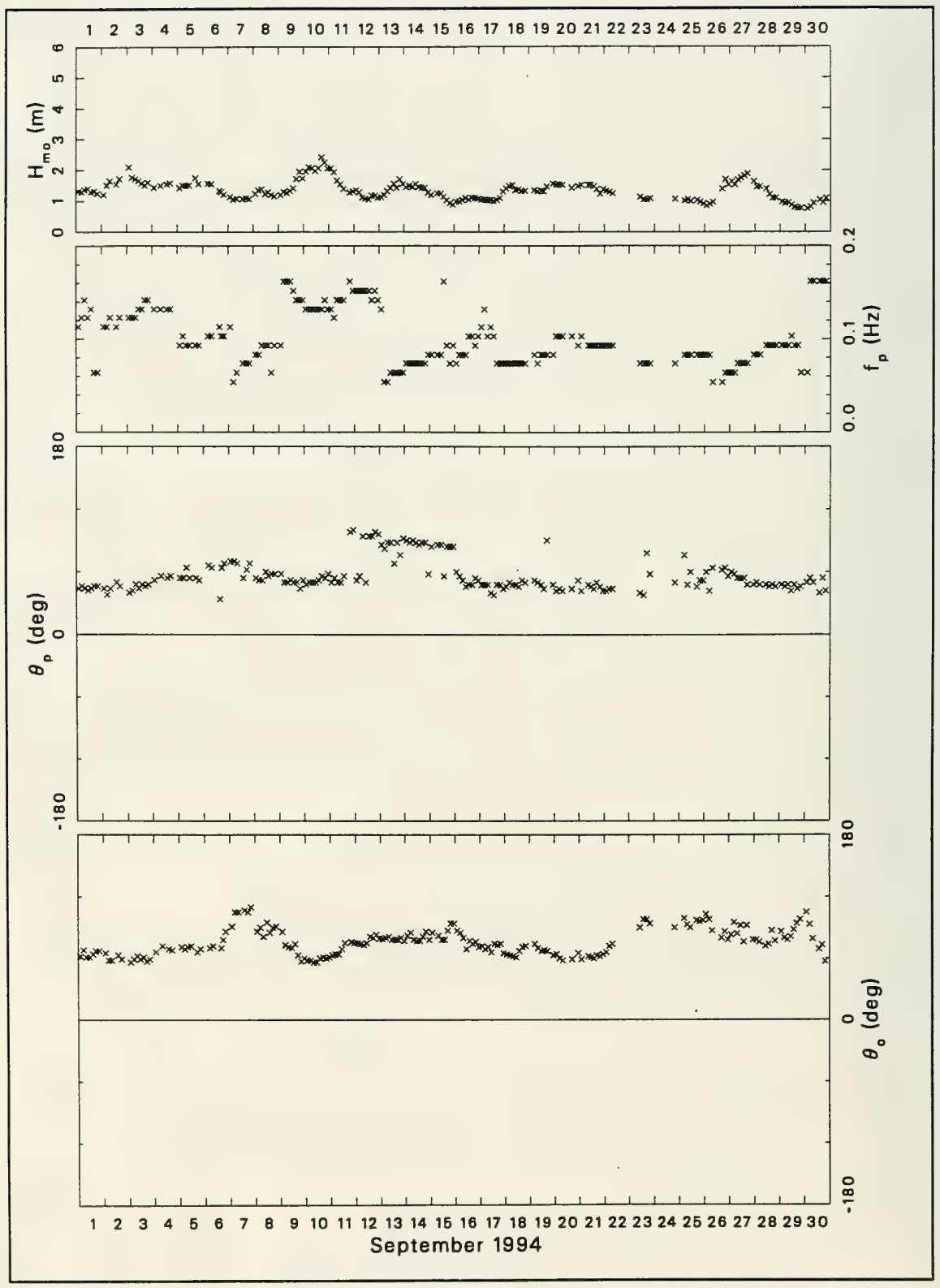

Figure B9. Bulk data for September 1994 (Continued) 


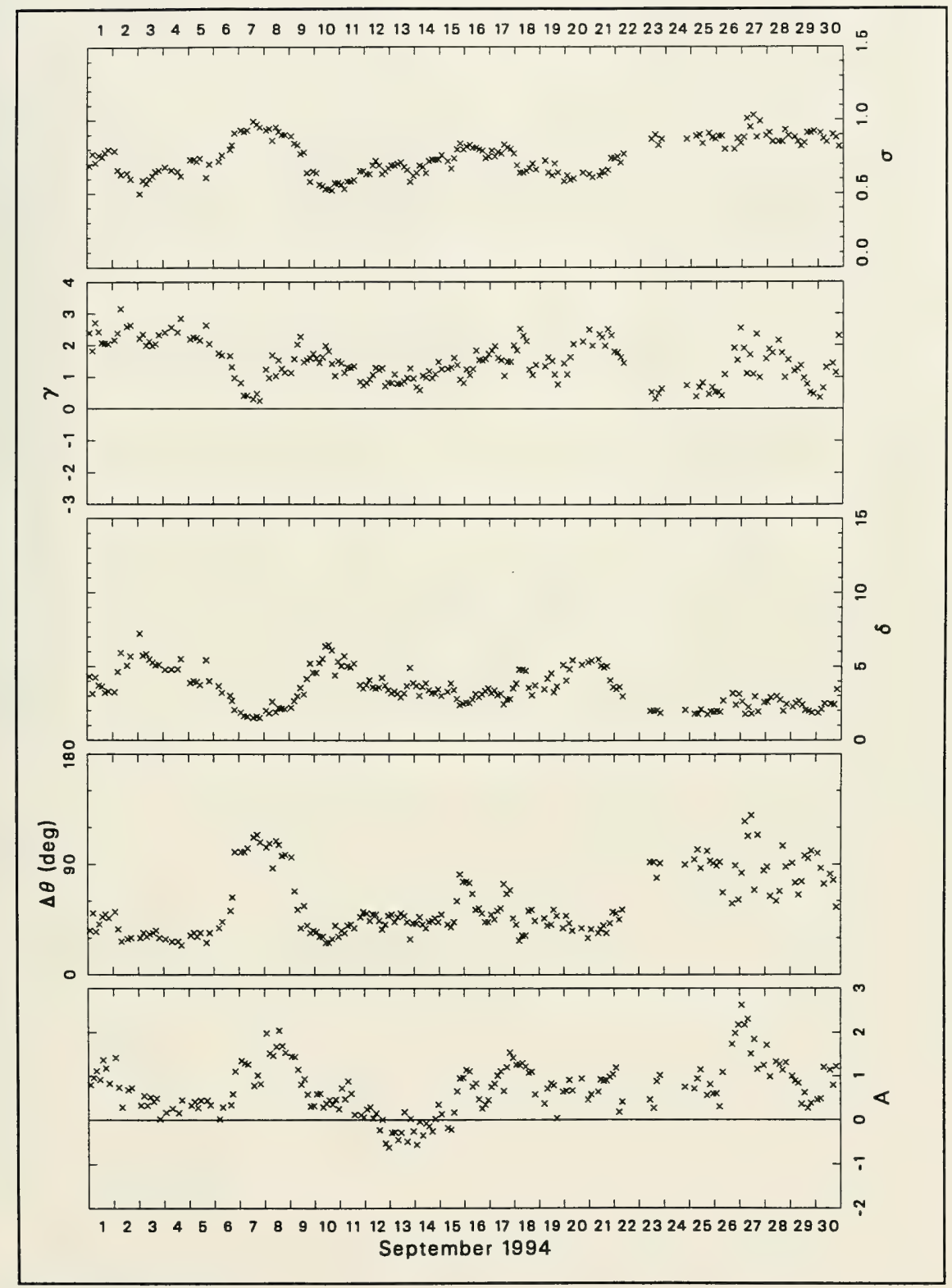

Figure B9. (Concluded) 


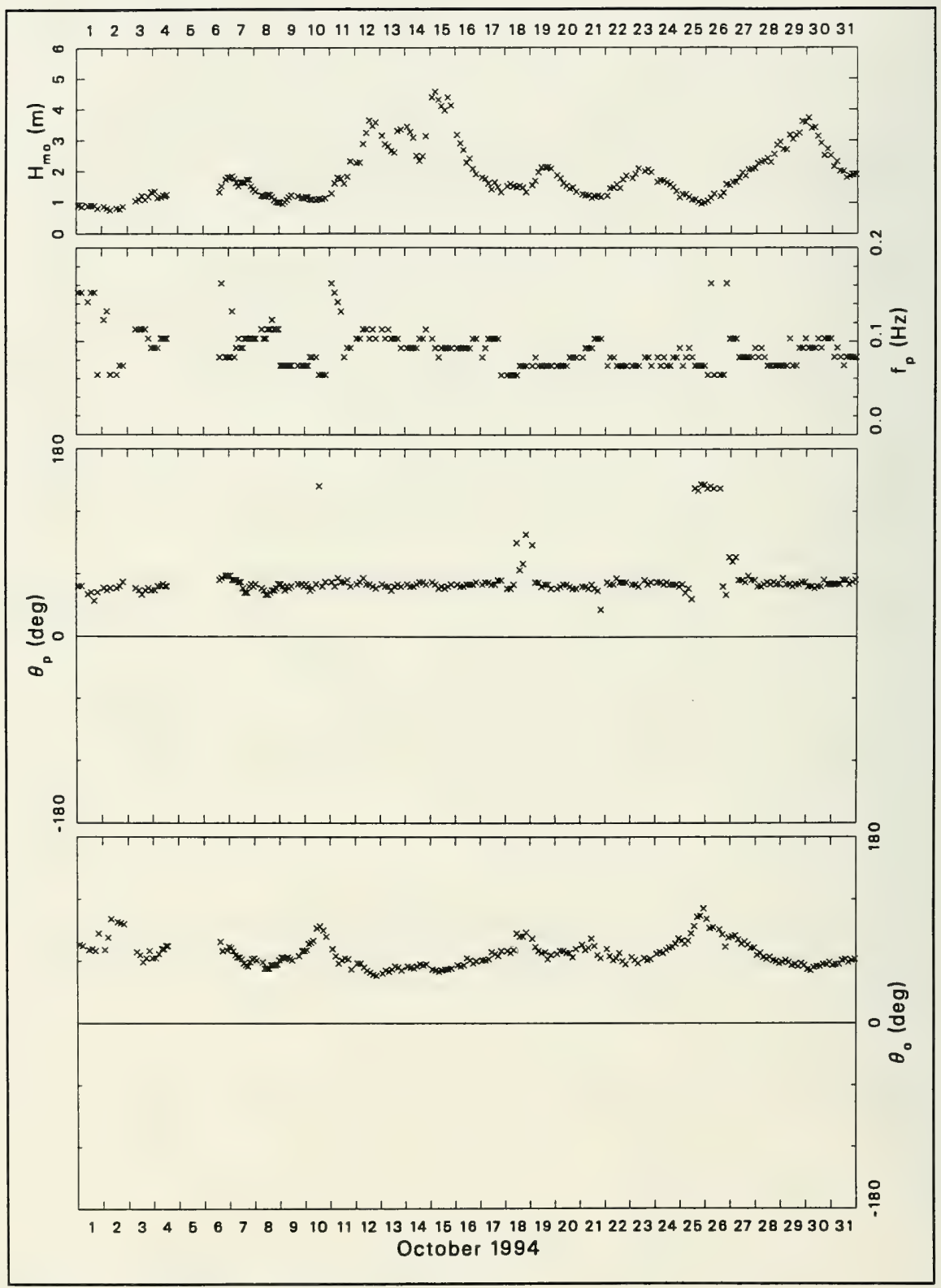

Figure B10. Bulk data for October 1994 (Continued) 


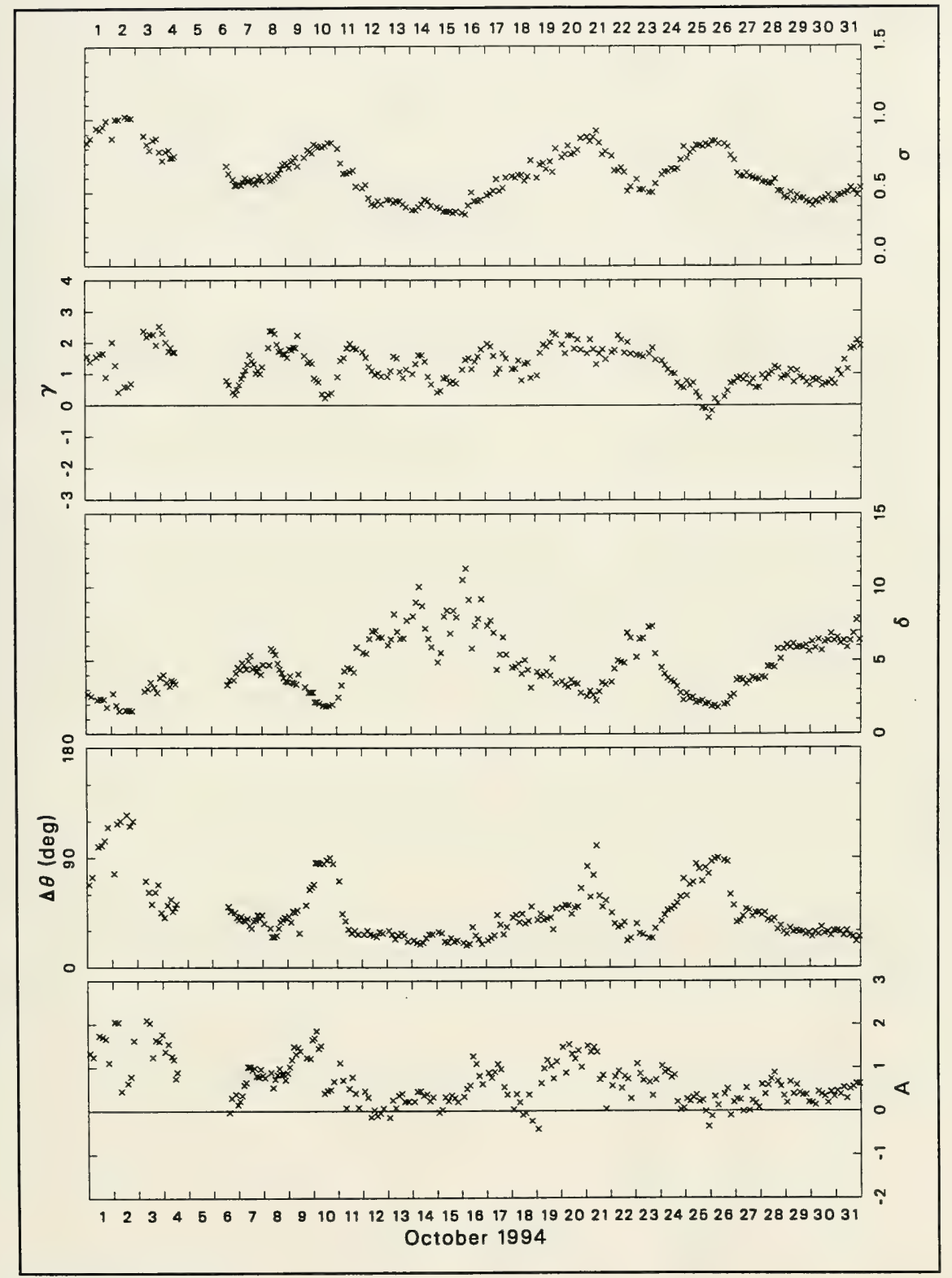

Figure B10. (Concluded) 


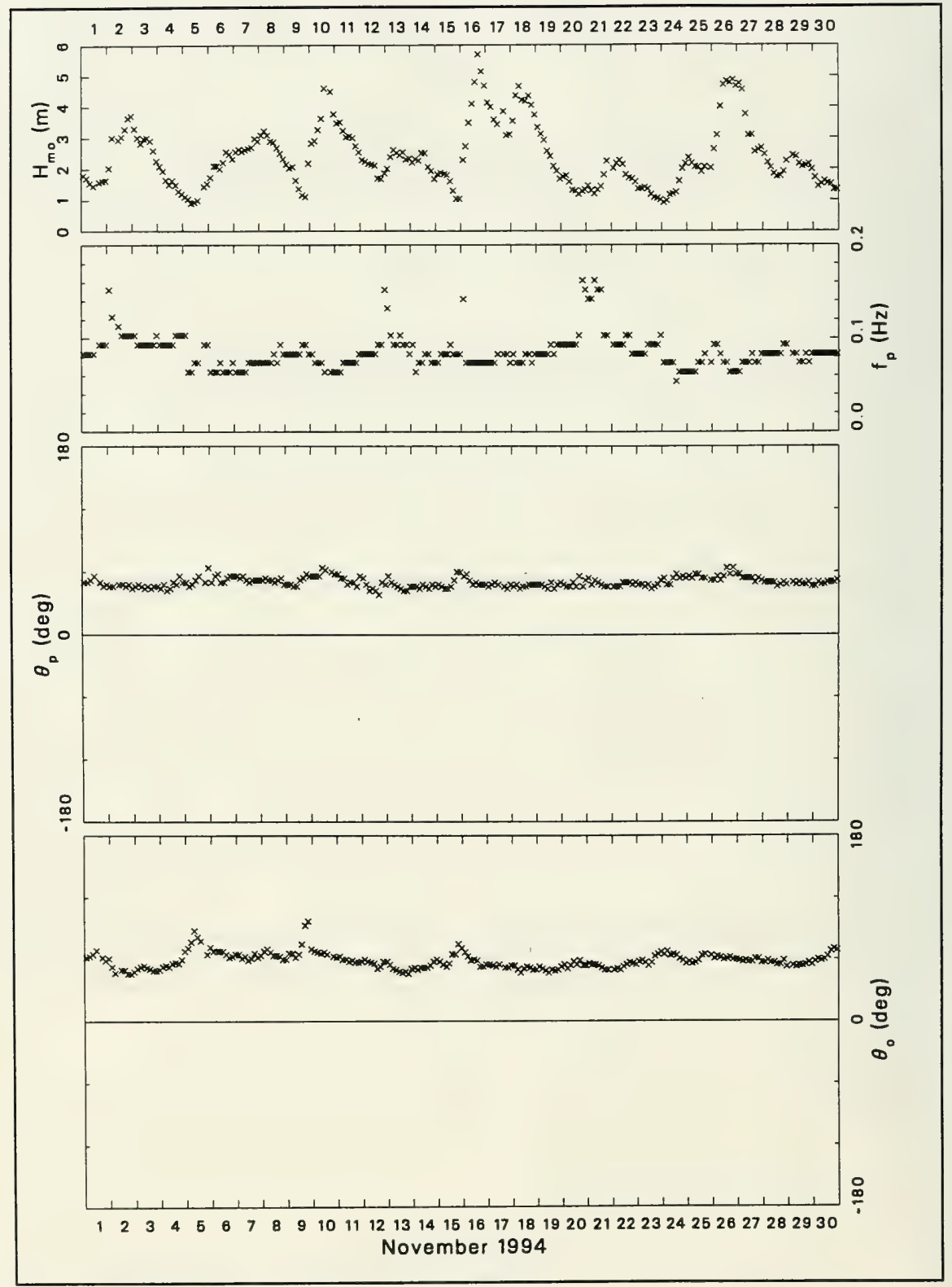

Figure B11. Bulk data for November 1994 (Continued) 


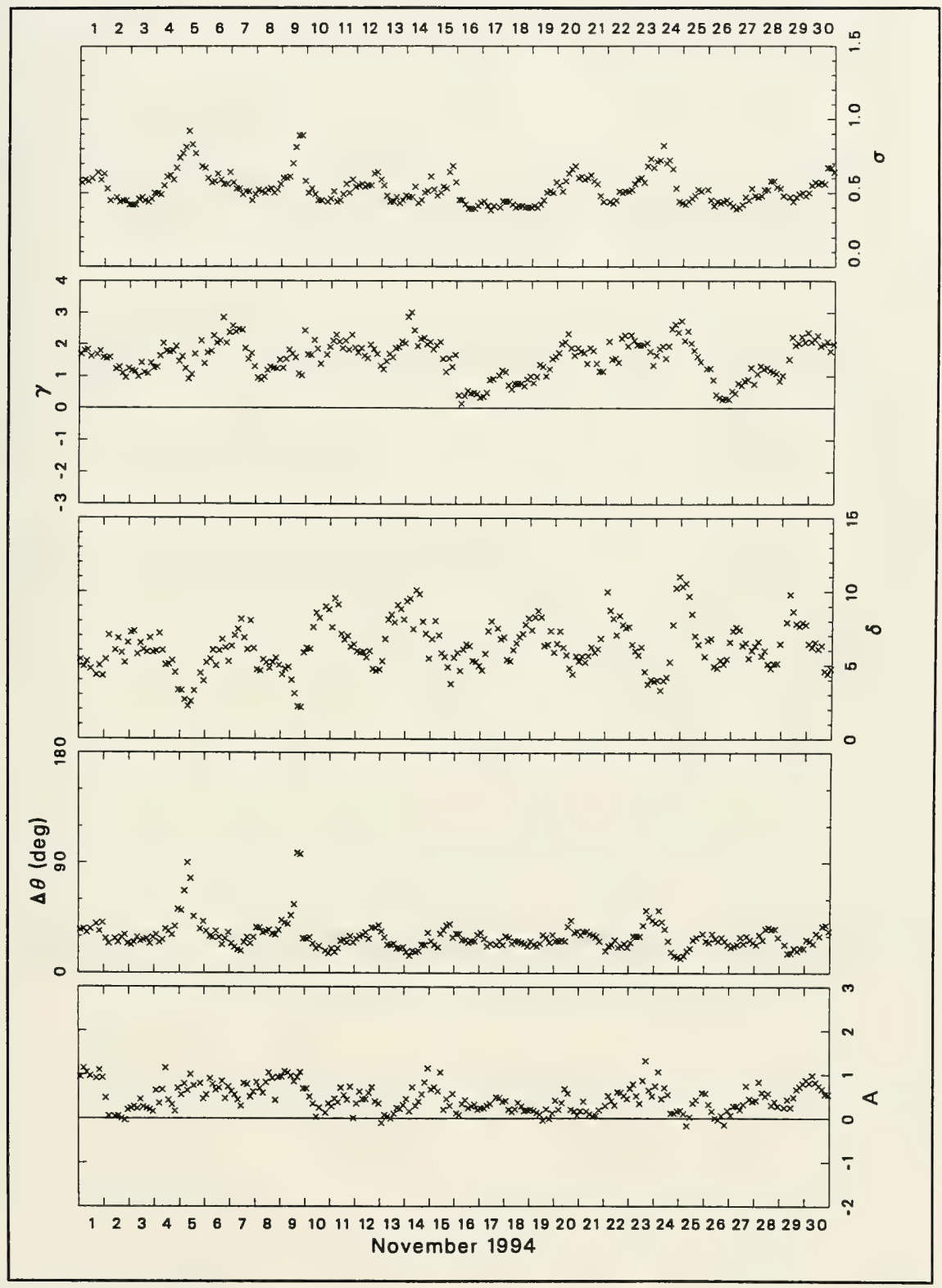

Figure B11. (Concluded) 


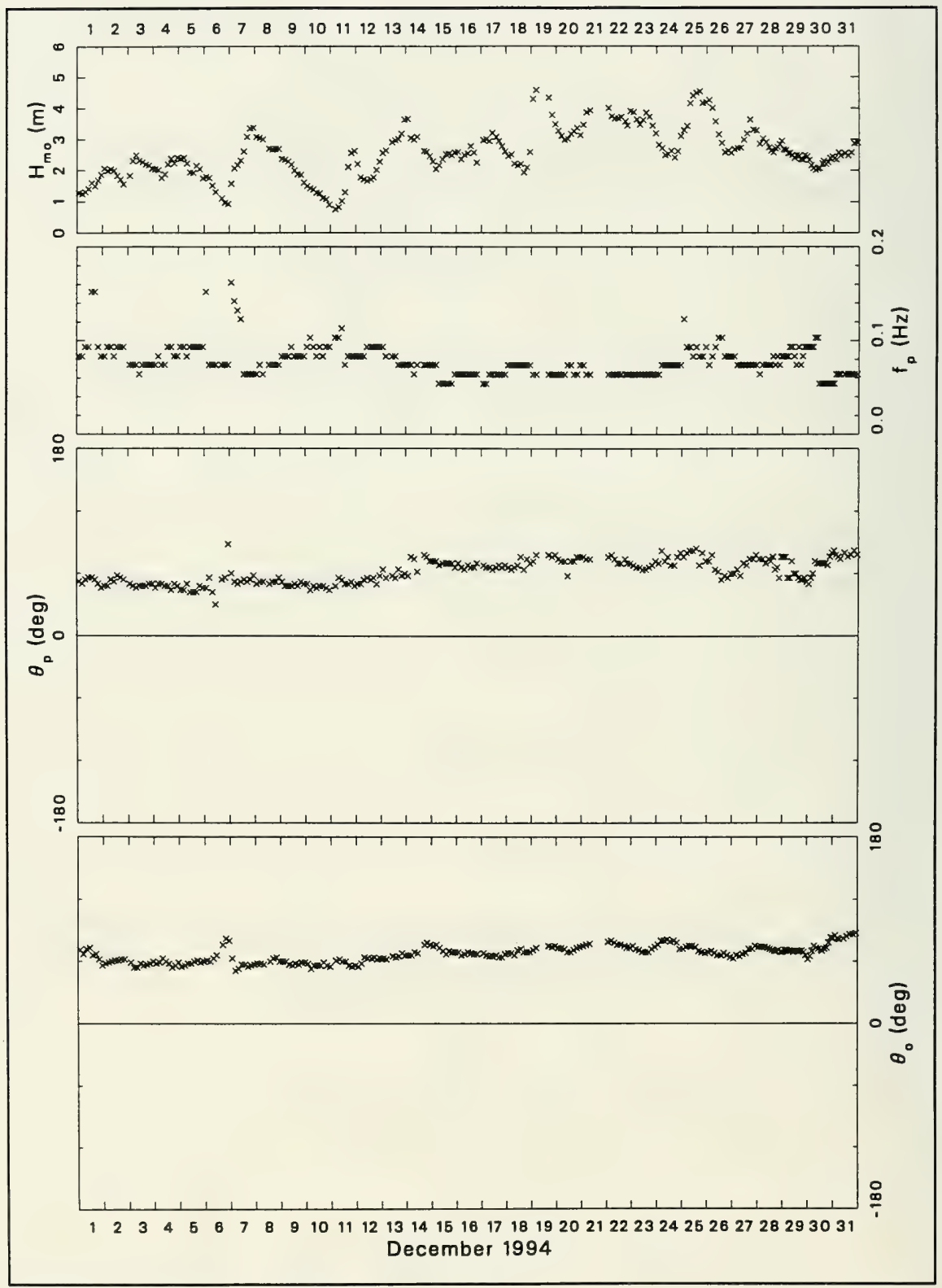

Figure B12. Bulk data for December 1994 (Continued) 


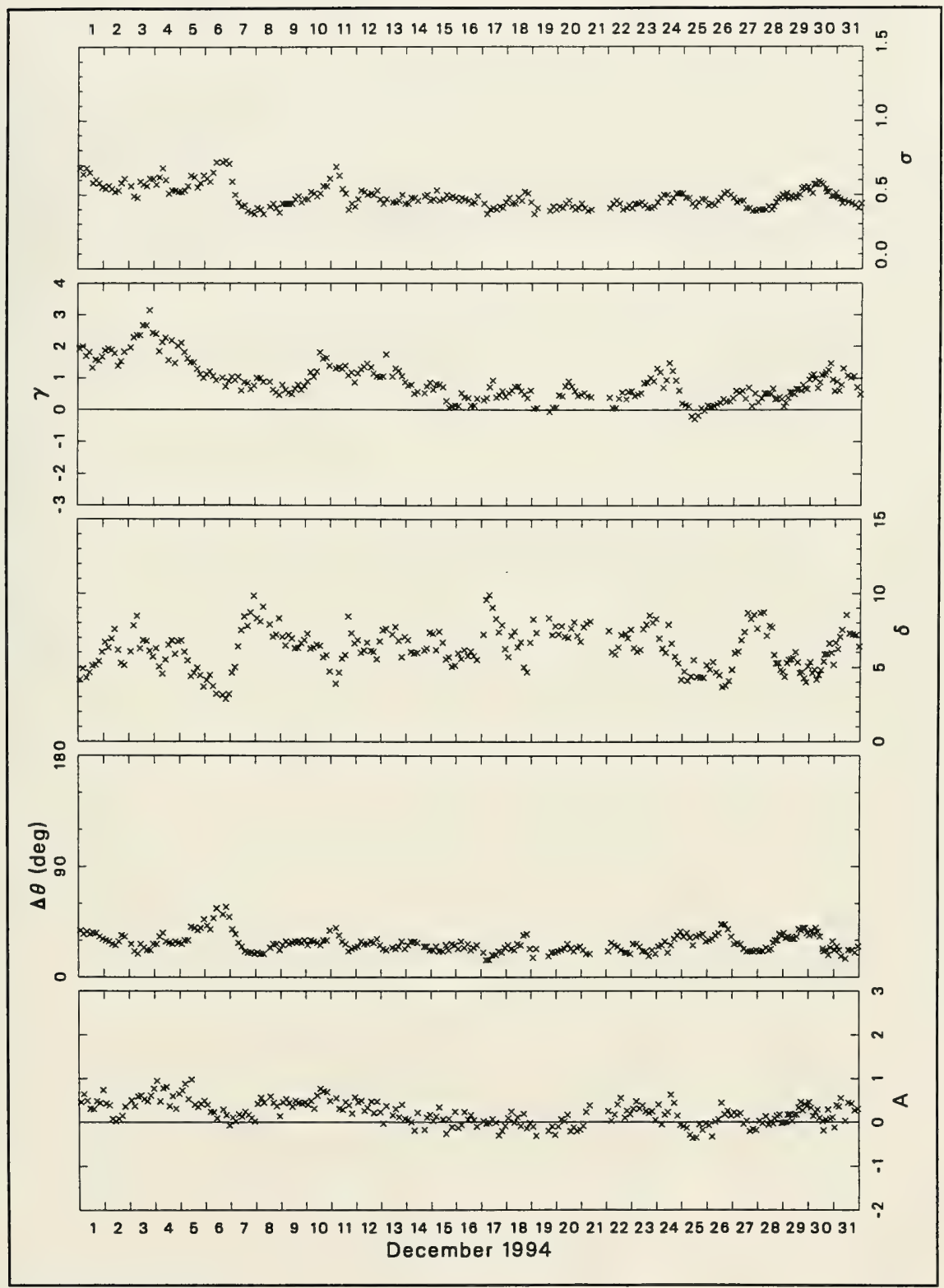

Figure B12. (Concluded) 



\section{Appendix C \\ Listing of FORTRAN Computer Program}

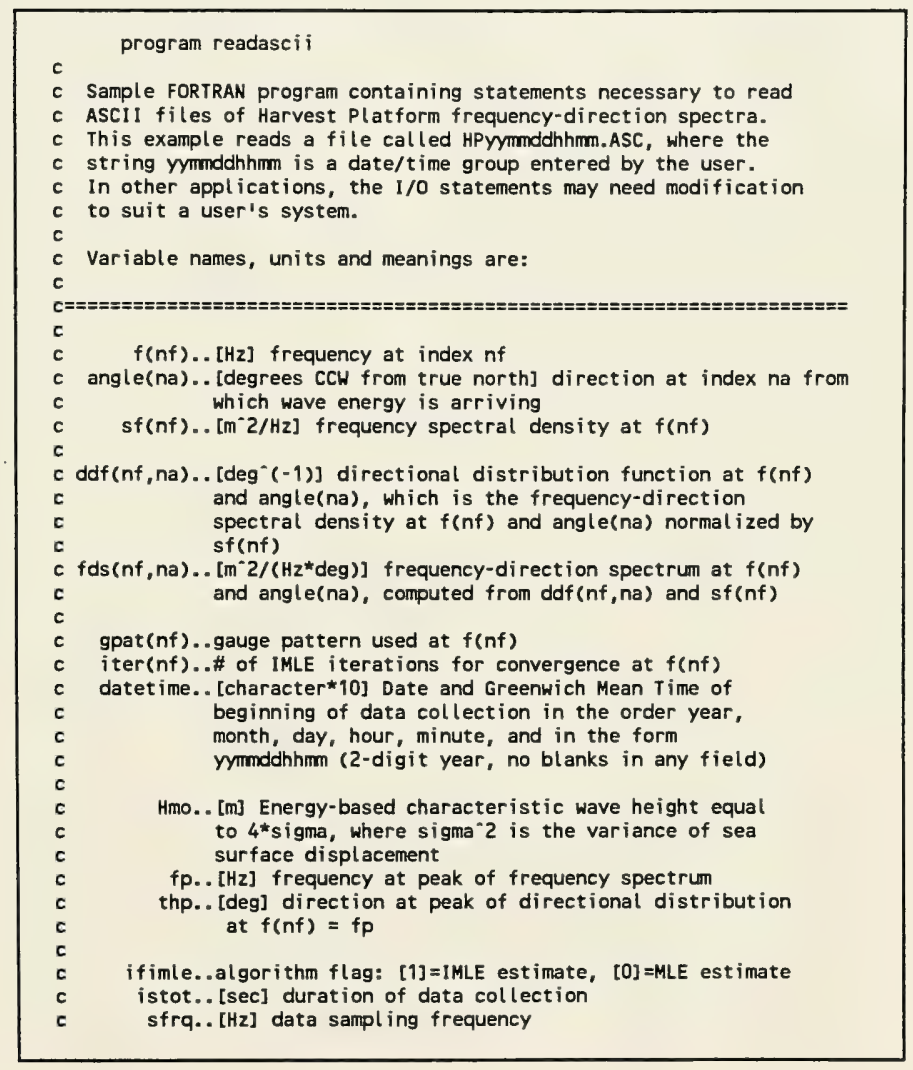

Figure C1. Listing of FORTRAN Computer Program (Sheet 1 of 3) 
if windo.. windowing $\mathrm{flag}$ : $[0]=$ no windowing of data segments, [1]=segments windowed (Kaiser-Bessel window)

ifdtrnd..detrending flag: [0]=no detrending, [1]=L inear trend removed from data segments

nfft.. \# of points in each data ensemble

nensb..\# of half-lapped segments of cross-spectral computations nband.. \# of raw frequency bands averaged in frequency smoothing idgfr..degrees of freedom in cross-spectral computations (based on contiguous segments only)

nfrq.. number of output frequency bands, equals range of index nf

delfs... [Hz] output frequency bandwidth

nang...number of output angle bins, equals range of index na delang... [deg] output angle bin width

dmin.. [m] minimum ensemble segment water depth at reference gauge 'rname' during collection

dbar... [m] mean water depth at gauge 'rname' during collection dmax... [m] maximum ensemble segment water depth at reference gauge 'rname' during collection

rname...[character*5] reference gauge id for depth computations

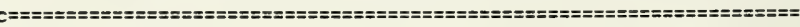

c

character ${ }^{*} 5$

character*6

character*10

character*80

dimension

dimension

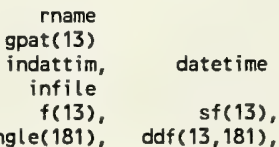

angle(181), iter (13)

fds $(13,181)$

c get file-naming date/time group from user

c

write(*,' (2x, ''Enter date/time group (ymmddhhmm)...'')')

read(*, '(a10)') indattim

! date/time string

define input data file

inf i le $=$ 'HP' $/ /$ indattim( $1: 10) / /$ ' . ASC'

open, read, and close data file

open (10, file=infile, status=' old' , form=' formatted' $)$

c

$\operatorname{read}(10$,

\& 'C a10, f10.2, f10.5, f10.1, i10, i10,

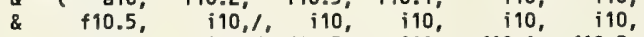

\& i10, i10, f10.5, i10, $1, f 10.1,+10.2$,

\& f10.2, f10.2, 5x, a5;')

\&atetime, Hmo, fp, thp, ifimle, istot,

\& sfrq, ifwindo, ifdtrnd, nfft, nensb, nband,

\& idgfr, nfra, delfs, nang, delang, dmin,

dbar, dmax, rname

$\operatorname{read}\left(10,1(10 f 8,1)^{\prime}\right)$ (angle(na), na=1, nang)

c

do $10 \mathrm{nf}=1, \mathrm{nfrq}$

read(10,

\& ' ( $\left.i 10, \quad f 10.5, \quad f 10.6, \quad 4 x, a 6, \quad i 10)^{\prime}\right)$

\& if, $f(n f), s f(n f), \operatorname{gpat}(n f), i \operatorname{ter}(n f)$

$\operatorname{read}\left(10, '(8 f 10.7)^{\prime}\right)(\operatorname{ddf}(n f, n a)$, na=1, nang)

10

cont inue

close(10)

Figure C1. (Sheet 2 of 3) 
c compute frequency-direction spectrum fds (nf, na) from ddf(nf, na)

c and $s f(n f)$

c

$$
\begin{aligned}
& \text { do } 20 n f=1 \text {, } n f r q \\
& \text { do } 25 \text { na=1, nang }
\end{aligned}
$$$$
f d s(n f, n a)=s f(n f) * d d f(n f, n a)
$$

25 continue

20 cont inue

$c$

c at this point, all relevant variables are defined and arrays

c are loaded; subsequent computations or operations can be done

c at the user's discretion...

c

end

Figure C1. (Sheet 3 of 3) 



\section{Appendix D Listing of Sample Data File}

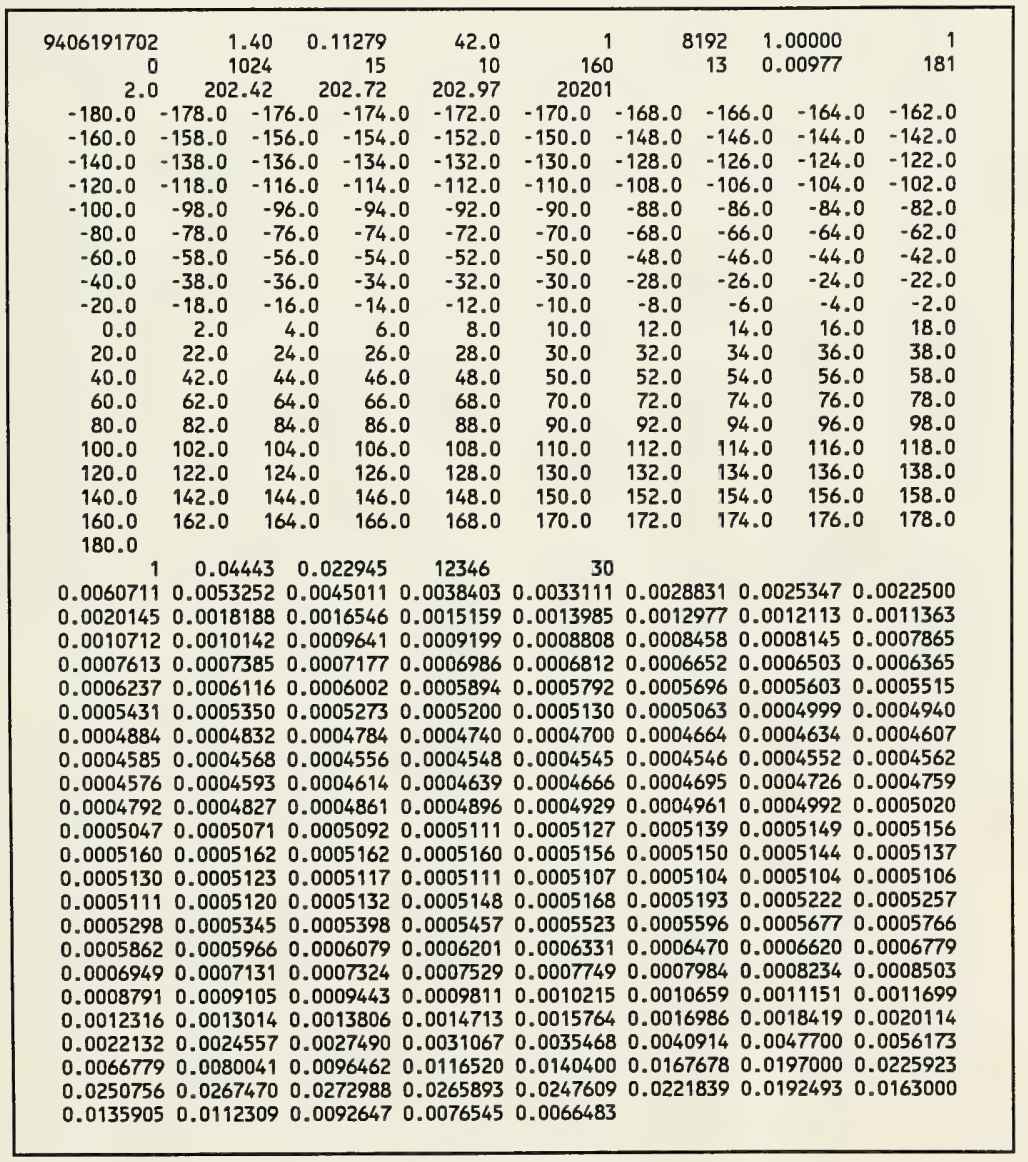

Figure D1. Listing of sample data file (Sheet 1 of 6 ) 
$\begin{array}{llllllllll}0.0193193 & 0.0184077 & 0.0170895 & 0.0153190 & 0.0133217 & 0.0112048 & 0.0092480 & 0.0074597\end{array}$ $\begin{array}{llllllllllll}0.0058946 & 0.0046099 & 0.0035386 & 0.0026957 & 0.0020745 & 0.0015988 & 0.0012458 & 0.0009987\end{array}$ $\begin{array}{lllllllllllll}0.0008208 & 0.0006947 & 0.0006082 & 0.0005547 & 0.0005174 & 0.0004952 & 0.0004832 & 0.0004781\end{array}$ $\begin{array}{llllllllllll}0.0004767 & 0.0004760 & 0.0004743 & 0.0004722 & 0.0004672 & 0.0004597 & 0.0004485 & 0.0004343\end{array}$ $\begin{array}{lllllllllllll}0.0004178 & 0.0003978 & 0.0003759 & 0.0003524 & 0.0003279 & 0.0003021 & 0.0002764 & 0.0002507\end{array}$ $\begin{array}{lllllllllll}0.0002256 & 0.0002014 & 0.0001782 & 0.0001564 & 0.0001364 & 0.0001181 & 0.0001016 & 0.0000871\end{array}$ $\begin{array}{llllllllllll}0.0000746 & 0.0000638 & 0.0000548 & 0.0000474 & 0.0000415 & 0.0000371 & 0.0000339 & 0.0000319\end{array}$ $\begin{array}{lllllllllll}0.0000309 & 0.0000311 & 0.0000324 & 0.0000350 & 0.0000390 & 0.0000445 & 0.0000520 & 0.0000617\end{array}$ $\begin{array}{llllllllllll}0.0000740 & 0.0000889 & 0.0001069 & 0.0001281 & 0.0001526 & 0.0001801 & 0.0002102 & 0.0002427\end{array}$ $\begin{array}{lllllllllllll}0.0002766 & 0.0003112 & 0.0003451 & 0.0003773 & 0.0004059 & 0.0004299 & 0.0004479 & 0.0004587\end{array}$ $\begin{array}{lllllllllll}0.0004614 & 0.0004556 & 0.0004421 & 0.0004222 & 0.0003980 & 0.0003713 & 0.0003449 & 0.0003211\end{array}$ $\begin{array}{llllllllllll}0.0003022 & 0.0002884 & 0.0002807 & 0.0002785 & 0.0002822 & 0.0002899 & 0.0003005 & 0.0003124\end{array}$ $\begin{array}{llllllllllll}0.0003246 & 0.0003357 & 0.0003445 & 0.0003507 & 0.0003529 & 0.0003513 & 0.0003463 & 0.0003375\end{array}$ $\begin{array}{llllllllllll}0.0003253 & 0.0003106 & 0.0002933 & 0.0002741 & 0.0002536 & 0.0002326 & 0.0002111 & 0.0001898\end{array}$ $\begin{array}{llllllllllll}0.0001692 & 0.0001495 & 0.0001312 & 0.0001145 & 0.0000993 & 0.0000861 & 0.0000748 & 0.0000654\end{array}$ $\begin{array}{llllllllllll}0.0000578 & 0.0000519 & 0.0000477 & 0.0000449 & 0.0000437 & 0.0000441 & 0.0000462 & 0.0000500\end{array}$ $\begin{array}{llllllllllll}0.0000562 & 0.0000649 & 0.0000769 & 0.0000927 & 0.0001132 & 0.0001392 & 0.0001719 & 0.0002123\end{array}$ $\begin{array}{llllllllllllll}0.0002609 & 0.0003193 & 0.0003886 & 0.0004682 & 0.0005592 & 0.0006618 & 0.0007741 & 0.0008986\end{array}$ $\begin{array}{lllllllllll}0.0010315 & 0.0011714 & 0.0013129 & 0.0014590 & 0.0016010 & 0.0017377 & 0.0018647 & 0.0019786\end{array}$ $\begin{array}{lllllllllll}0.0020822 & 0.0021782 & 0.0022795 & 0.0023725 & 0.0025086 & 0.0026904 & 0.0029601 & 0.0033376\end{array}$ $\begin{array}{lllllllllll}0.0038572 & 0.0045843 & 0.0055141 & 0.0067018 & 0.0081353 & 0.0097832 & 0.0115735 & 0.0134692\end{array}$ $\begin{array}{lllllllllll}0.0153736 & 0.0171945 & 0.0187583 & 0.0198669 & 0.0205504 & 0.0208362 & 0.0209647 & 0.0207193\end{array}$

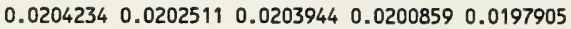

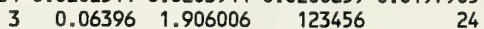

$\begin{array}{lllllllllll}0.0266505 & 0.0240051 & 0.0199338 & 0.0156902 & 0.0118319 & 0.0085920 & 0.0061034 & 0.0042657\end{array}$ $\begin{array}{lllllllllllll}0.0029355 & 0.0020195 & 0.0013950 & 0.0009715 & 0.0006931 & 0.0005074 & 0.0003862 & 0.0003071\end{array}$ $\begin{array}{lllllllllll}0.0002563 & 0.0002242 & 0.0002057 & 0.0001973 & 0.0001957 & 0.0001998 & 0.0002076 & 0.0002187\end{array}$ $\begin{array}{lllllllllllllll}0.0002319 & 0.0002457 & 0.0002591 & 0.0002714 & 0.0002818 & 0.0002895 & 0.0002941 & 0.0002953\end{array}$ $\begin{array}{lllllllllllllllll}0.0002931 & 0.0002871 & 0.0002781 & 0.0002661 & 0.0002517 & 0.0002351 & 0.0002171 & 0.0001980\end{array}$ $\begin{array}{llllllllllll}0.0001784 & 0.0001588 & 0.0001396 & 0.0001213 & 0.0001041 & 0.0000884 & 0.0000744 & 0.0000621\end{array}$ $\begin{array}{lllllllllll}0.0000515 & 0.0000427 & 0.0000354 & 0.0000296 & 0.0000251 & 0.0000218 & 0.0000194 & 0.0000179\end{array}$ $\begin{array}{lllllllllll}0.0000172 & 0.0000173 & 0.0000181 & 0.0000198 & 0.0000226 & 0.0000267 & 0.0000323 & 0.0000399\end{array}$ $\begin{array}{lllllllllll}0.0000498 & 0.0000626 & 0.0000786 & 0.0000983 & 0.0001219 & 0.0001494 & 0.0001806 & 0.0002148\end{array}$ $\begin{array}{lllllllllll}0.0002510 & 0.0002879 & 0.0003233 & 0.0003552 & 0.0003810 & 0.0003983 & 0.0004054 & 0.0004011\end{array}$ $\begin{array}{llllllllllll}0.0003856 & 0.0003603 & 0.0003282 & 0.0002926 & 0.0002570 & 0.0002241 & 0.0001961 & 0.0001735\end{array}$ $\begin{array}{llllllllllll}0.0001565 & 0.0001446 & 0.0001368 & 0.0001324 & 0.0001307 & 0.0001309 & 0.0001322 & 0.0001341\end{array}$ $\begin{array}{llllllllllll}0.0001361 & 0.0001380 & 0.0001392 & 0.0001397 & 0.0001394 & 0.0001381 & 0.0001359 & 0.0001327\end{array}$ $\begin{array}{lllllllllll}0.0001286 & 0.0001238 & 0.0001183 & 0.0001121 & 0.0001055 & 0.0000985 & 0.0000912 & 0.0000838\end{array}$ $\begin{array}{llllllllllll}0.0000764 & 0.0000690 & 0.0000619 & 0.0000550 & 0.0000486 & 0.0000426 & 0.0000371 & 0.0000323\end{array}$ $\begin{array}{llllllllllllll}0.0000281 & 0.0000245 & 0.0000215 & 0.0000191 & 0.0000173 & 0.0000161 & 0.0000155 & 0.0000155\end{array}$ $\begin{array}{lllllllllllllll}0.0000162 & 0.0000177 & 0.0000202 & 0.0000241 & 0.0000301 & 0.0000388 & 0.0000515 & 0.0000698\end{array}$ $\begin{array}{lllllllllllll}0.0000961 & 0.0001333 & 0.0001855 & 0.0002573 & 0.0003543 & 0.0004831 & 0.0006498 & 0.0008611\end{array}$ $\begin{array}{lllllllllll}0.0011224 & 0.0014354 & 0.0017974 & 0.0022041 & 0.0026385 & 0.0030759 & 0.0034895 & 0.0038434\end{array}$ $\begin{array}{lllllllllll}0.0041111 & 0.0042729 & 0.0043422 & 0.0043300 & 0.0042985 & 0.0042918 & 0.0043646 & 0.0045543\end{array}$ $\begin{array}{lllllllllll}0.0048808 & 0.0053739 & 0.0060164 & 0.0068127 & 0.0077325 & 0.0087527 & 0.0098372 & 0.0109554\end{array}$ $\begin{array}{llllllllll}0.0120929 & 0.0132428 & 0.0143793 & 0.0155102 & 0.0166772 & 0.0179936 & 0.0196328 & 0.0216170\end{array}$ $\begin{array}{lllllll}0.0239779 & 0.0263410 & 0.0282539 & 0.0286674 & 0.0278960\end{array}$

$$
\begin{array}{lllll}
4 & 0.07373 & 0.278954 & 123456 & 30
\end{array}
$$

$\begin{array}{llllllllllllll}0.0187927 & 0.0190464 & 0.0183675 & 0.0164729 & 0.0137951 & 0.0108111 & 0.0079991 & 0.0056283\end{array}$ $\begin{array}{llllllllllll}0.0038047 & 0.0025047 & 0.0016247 & 0.0010542 & 0.0006957 & 0.0004728 & 0.0003356 & 0.0002509\end{array}$ $\begin{array}{lllllllllll}0.0001988 & 0.0001669 & 0.0001483 & 0.0001388 & 0.0001354 & 0.0001368 & 0.0001416 & 0.0001492\end{array}$ $\begin{array}{llllllllll}0.0001587 & 0.0001696 & 0.0001812 & 0.0001928 & 0.0002039 & 0.0002139 & 0.0002224 & 0.0002290\end{array}$ $\begin{array}{lllllllllll}0.0002334 & 0.0002354 & 0.0002349 & 0.0002319 & 0.0002265 & 0.0002189 & 0.0002093 & 0.0001979\end{array}$ $\begin{array}{lllllllllll}0.0001851 & 0.0001712 & 0.0001566 & 0.0001416 & 0.0001266 & 0.0001120 & 0.0000981 & 0.0000851\end{array}$ $\begin{array}{lllllllllllll}0.0000732 & 0.0000627 & 0.0000535 & 0.0000457 & 0.0000393 & 0.0000341 & 0.0000303 & 0.0000276\end{array}$ $\begin{array}{lllllllllll}0.0000260 & 0.0000255 & 0.0000262 & 0.0000282 & 0.0000317 & 0.0000374 & 0.0000456 & 0.0000572\end{array}$ $\begin{array}{llllllllllll}0.0000731 & 0.0000943 & 0.0001215 & 0.0001551 & 0.0001951 & 0.0002403 & 0.0002887 & 0.0003374\end{array}$ $\begin{array}{llllllllll}0.0003825 & 0.0004204 & 0.0004472 & 0.0004604 & 0.0004586 & 0.0004418 & 0.0004119 & 0.0003720\end{array}$ $\begin{array}{lllllllllll}0.0003257 & 0.0002771 & 0.0002299 & 0.0001868 & 0.0001495 & 0.0001189 & 0.0000948 & 0.0000765\end{array}$ $\begin{array}{llllllllllll}0.0000633 & 0.0000542 & 0.0000484 & 0.0000454 & 0.0000448 & 0.0000464 & 0.0000501 & 0.0000561\end{array}$ $\begin{array}{lllllllllll}0.0000646 & 0.0000758 & 0.0000899 & 0.0001070 & 0.0001270 & 0.0001499 & 0.0001751 & 0.0002023\end{array}$ $\begin{array}{llllllllllll}0.0002304 & 0.0002588 & 0.0002862 & 0.0003116 & 0.0003340 & 0.0003522 & 0.0003656 & 0.0003733\end{array}$ $\begin{array}{lllllllllll}0.0003749 & 0.0003705 & 0.0003602 & 0.0003446 & 0.0003248 & 0.0003018 & 0.0002772 & 0.0002522\end{array}$ $\begin{array}{llllllllllll}0.0002282 & 0.0002066 & 0.0001883 & 0.0001741 & 0.0001645 & 0.0001600 & 0.0001613 & 0.0001689\end{array}$ $\begin{array}{llllllllll}0.0001841 & 0.0002080 & 0.0002423 & 0.0002890 & 0.0003499 & 0.0004262 & 0.0005190 & 0.0006275\end{array}$ $\begin{array}{llllllllll}0.0007497 & 0.0008823 & 0.0010206 & 0.0011580 & 0.0012886 & 0.0014057 & 0.0015033 & 0.0015772\end{array}$

Figure D1. (Sheet 2 of 6 ) 
$\begin{array}{lllllllllll}0.0016238 & 0.0016417 & 0.0016315 & 0.0015962 & 0.0015406 & 0.0014710 & 0.0013957 & 0.0013233\end{array}$ $\begin{array}{llllllllllll}0.0012640 & 0.0012283 & 0.0012298 & 0.0012818 & 0.0014097 & 0.0016472 & 0.0020501 & 0.0027031\end{array}$ $\begin{array}{lllllllllll}0.0037305 & 0.0052903 & 0.0075380 & 0.0105780 & 0.0143231 & 0.0184371 & 0.0223070 & 0.0251846\end{array}$ $\begin{array}{lllllllllll}0.0264517 & 0.0258812 & 0.0237446 & 0.0206977 & 0.0175358 & 0.0148847 & 0.0131475 & 0.0123664\end{array}$ $\begin{array}{llllll}0.0125511 & 0.0135672 & 0.0152252 & 0.0170694 & 0.0182673\end{array}$

$\begin{array}{lrrrr}5 & 0.08350 & 0.103069 & 123456 & 17\end{array}$

$\begin{array}{llllllllllllll}0.0073335 & 0.0070561 & 0.0065963 & 0.0060441 & 0.0054270 & 0.0047748 & 0.0041188 & 0.0034858\end{array}$ $\begin{array}{llllllllllll}0.0028977 & 0.0023706 & 0.0019132 & 0.0015284 & 0.0012139 & 0.0009635 & 0.0007686 & 0.0006200\end{array}$ $\begin{array}{llllllllllll}0.0005086 & 0.0004262 & 0.0003662 & 0.0003230 & 0.0002924 & 0.0002712 & 0.0002570 & 0.0002478\end{array}$ $\begin{array}{llllllllllll}0.0002422 & 0.0002391 & 0.0002377 & 0.0002371 & 0.0002370 & 0.0002369 & 0.0002365 & 0.0002356\end{array}$ $\begin{array}{lllllllllll}0.0002341 & 0.0002320 & 0.0002291 & 0.0002255 & 0.0002212 & 0.0002162 & 0.0002106 & 0.0002044\end{array}$ $\begin{array}{lllllllllll}0.0001976 & 0.0001904 & 0.0001827 & 0.0001746 & 0.0001662 & 0.0001575 & 0.0001486 & 0.0001397\end{array}$ $\begin{array}{lllllllllll}0.0001307 & 0.0001219 & 0.0001134 & 0.0001052 & 0.0000975 & 0.0000905 & 0.0000842 & 0.0000788\end{array}$ $\begin{array}{lllllllllll}0.0000745 & 0.0000712 & 0.0000691 & 0.0000683 & 0.0000688 & 0.0000709 & 0.0000745 & 0.0000799\end{array}$ $\begin{array}{llllllllllll}0.0000871 & 0.0000963 & 0.0001076 & 0.0001210 & 0.0001364 & 0.0001539 & 0.0001733 & 0.0001942\end{array}$ $\begin{array}{lllllllllll}0.0002163 & 0.0002394 & 0.0002630 & 0.0002868 & 0.0003102 & 0.0003330 & 0.0003548 & 0.0003751\end{array}$ $\begin{array}{lllllllllllll}0.0003937 & 0.0004103 & 0.0004245 & 0.0004362 & 0.0004451 & 0.0004513 & 0.0004547 & 0.0004555\end{array}$ $\begin{array}{llllllllllll}0.0004542 & 0.0004513 & 0.0004477 & 0.0004445 & 0.0004434 & 0.0004459 & 0.0004542 & 0.0004706\end{array}$ $\begin{array}{llllllllllll}0.0004979 & 0.0005394 & 0.0005985 & 0.0006792 & 0.0007854 & 0.0009206 & 0.0010873 & 0.0012860\end{array}$ $\begin{array}{lllllllllllll}0.0015145 & 0.0017678 & 0.0020376 & 0.0023131 & 0.0025812 & 0.0028281 & 0.0030396 & 0.0032030\end{array}$ $\begin{array}{llllllllll}0.0033082 & 0.0033487 & 0.0033229 & 0.0032339 & 0.0030909 & 0.0029076 & 0.0027012 & 0.0024895\end{array}$ $\begin{array}{lllllllllllll}0.0022896 & 0.0021149 & 0.0019749 & 0.0018742 & 0.0018141 & 0.0017931 & 0.0018074 & 0.0018518\end{array}$ $\begin{array}{llllllllllll}0.0019211 & 0.0020088 & 0.0021090 & 0.0022160 & 0.0023246 & 0.0024301 & 0.0025296 & 0.0026200\end{array}$ $\begin{array}{lllllllllll}0.0026992 & 0.0027662 & 0.0028201 & 0.0028604 & 0.0028874 & 0.0029019 & 0.0029051 & 0.0028996\end{array}$ $\begin{array}{llllllllll}0.0028888 & 0.0028778 & 0.0028732 & 0.0028845 & 0.0029238 & 0.0030063 & 0.0031510 & 0.0033817\end{array}$ $\begin{array}{llllllllllll}0.0037271 & 0.0042199 & 0.0048956 & 0.0057851 & 0.0069087 & 0.0082621 & 0.0098082 & 0.0114669\end{array}$ $\begin{array}{llllllllllll}0.0131227 & 0.0146364 & 0.0158607 & 0.0166739 & 0.0169917 & 0.0167923 & 0.0161154 & 0.0150639\end{array}$ $\begin{array}{lllllllllll}0.0137799 & 0.0124221 & 0.0111354 & 0.0100286 & 0.0091614 & 0.0085497 & 0.0081684 & 0.0079664\end{array}$ $\begin{array}{llllll}0.0078785 & 0.0078352 & 0.0077734 & 0.0076412 & 0.0074798\end{array}$

$$
\begin{array}{lllll}
6 & 0.09326 & 0.147455 & 123456 & 30
\end{array}
$$

$\begin{array}{llllllllllllllll}0.0008755 & 0.0008897 & 0.0009024 & 0.0009057 & 0.0008983 & 0.0008792 & 0.0008484 & 0.0008063\end{array}$ $\begin{array}{lllllllllllll}0.0007543 & 0.0006942 & 0.0006284 & 0.0005599 & 0.0004914 & 0.0004257 & 0.0003652 & 0.0003113\end{array}$ $\begin{array}{lllllllllll}0.0002651 & 0.0002267 & 0.0001956 & 0.0001711 & 0.0001523 & 0.0001383 & 0.0001279 & 0.0001206\end{array}$ $\begin{array}{llllllllll}0.0001155 & 0.0001120 & 0.0001098 & 0.0001083 & 0.0001072 & 0.0001063 & 0.0001054 & 0.0001042\end{array}$ $\begin{array}{lllllllllllll}0.0001028 & 0.0001011 & 0.0000990 & 0.0000965 & 0.0000937 & 0.0000906 & 0.0000871 & 0.0000835\end{array}$ $\begin{array}{llllllllllllll}0.0000797 & 0.0000757 & 0.0000716 & 0.0000674 & 0.0000632 & 0.0000590 & 0.0000548 & 0.0000507\end{array}$ $\begin{array}{llllllllllll}0.0000467 & 0.0000428 & 0.0000391 & 0.0000356 & 0.0000322 & 0.0000292 & 0.0000263 & 0.0000238\end{array}$ $\begin{array}{llllllllll}0.0000215 & 0.0000195 & 0.0000178 & 0.0000165 & 0.0000154 & 0.0000147 & 0.0000143 & 0.0000142\end{array}$ $\begin{array}{llllllllll}0.0000146 & 0.0000153 & 0.0000166 & 0.0000185 & 0.0000212 & 0.0000248 & 0.0000295 & 0.0000356\end{array}$ $\begin{array}{llllllllllll}0.0000434 & 0.0000531 & 0.0000651 & 0.0000795 & 0.0000965 & 0.0001163 & 0.0001388 & 0.0001639\end{array}$ $\begin{array}{lllllllllll}0.0001915 & 0.0002211 & 0.0002525 & 0.0002850 & 0.0003181 & 0.0003512 & 0.0003837 & 0.0004149\end{array}$ $\begin{array}{lllllllllll}0.0004442 & 0.0004710 & 0.0004949 & 0.0005155 & 0.0005329 & 0.0005472 & 0.0005592 & 0.0005701\end{array}$ $\begin{array}{lllllllllll}0.0005820 & 0.0005977 & 0.0006217 & 0.0006602 & 0.0007226 & 0.0008233 & 0.0009862 & 0.0012513\end{array}$ $\begin{array}{llllllllllll}0.0016878 & 0.0024144 & 0.0036289 & 0.0056390 & 0.0088647 & 0.0137484 & 0.0204630 & 0.0284677\end{array}$ $\begin{array}{llllllllll}0.0361113 & 0.0409808 & 0.0411608 & 0.0366926 & 0.0296047 & 0.0224047 & 0.0166240 & 0.0126025\end{array}$ $\begin{array}{llllllllll}0.0100203 & 0.0084386 & 0.0074917 & 0.0069189 & 0.0065563 & 0.0062955 & 0.0060652 & 0.0058236\end{array}$ $\begin{array}{llllllllllll}0.0055473 & 0.0052261 & 0.0048608 & 0.0044582 & 0.0040292 & 0.0035867 & 0.0031455 & 0.0027193\end{array}$ $\begin{array}{llllllllllll}0.0023202 & 0.0019580 & 0.0016399 & 0.0013690 & 0.0011461 & 0.0009690 & 0.0008341 & 0.0007367\end{array}$ $\begin{array}{llllllllllll}0.0006726 & 0.0006379 & 0.0006298 & 0.0006470 & 0.0006888 & 0.0007554 & 0.0008469 & 0.0009633\end{array}$ $\begin{array}{lllllllllll}0.0011036 & 0.0012652 & 0.0014438 & 0.0016328 & 0.0018238 & 0.0020067 & 0.0021697 & 0.0023011\end{array}$ $\begin{array}{llllllllll}0.0023893 & 0.0024251 & 0.0024026 & 0.0023213 & 0.0021866 & 0.0020097 & 0.0018068 & 0.0015960\end{array}$ $\begin{array}{lllllllllll}0.0013945 & 0.0012153 & 0.0010665 & 0.0009510 & 0.0008678 & 0.0008136 & 0.0007841 & 0.0007745\end{array}$ $\begin{array}{llllll}0.0007803 & 0.0007968 & 0.0008199 & 0.0008454 & 0.0008641\end{array}$

$$
\begin{array}{lllll}
7 & 0.10303 & 0.693095 & 123456 & 30
\end{array}
$$

$\begin{array}{lllllllllll}0.0002433 & 0.0002404 & 0.0002354 & 0.0002293 & 0.0002224 & 0.0002150 & 0.0002072 & 0.0001992\end{array}$ $\begin{array}{lllllllllll}0.0001911 & 0.0001829 & 0.0001746 & 0.0001664 & 0.0001581 & 0.0001497 & 0.0001414 & 0.0001332\end{array}$ $\begin{array}{lllllllllllll}0.0001251 & 0.0001172 & 0.0001096 & 0.0001025 & 0.0000957 & 0.0000895 & 0.0000838 & 0.0000787\end{array}$ $\begin{array}{llllllllllll}0.0000740 & 0.0000698 & 0.0000659 & 0.0000624 & 0.0000592 & 0.0000562 & 0.0000534 & 0.0000507\end{array}$ $\begin{array}{lllllllllllll}0.0000482 & 0.0000459 & 0.0000437 & 0.0000416 & 0.0000396 & 0.0000377 & 0.0000360 & 0.0000344\end{array}$ $\begin{array}{llllllllllll}0.0000328 & 0.0000314 & 0.0000301 & 0.0000289 & 0.0000278 & 0.0000267 & 0.0000258 & 0.0000249\end{array}$ $\begin{array}{lllllllll}0.0000240 & 0.0000232 & 0.0000225 & 0.0000218 & 0.0000212 & 0.0000206 & 0.0000200 & 0.0000194\end{array}$ $\begin{array}{llllllllllll}0.0000189 & 0.0000185 & 0.0000180 & 0.0000176 & 0.0000173 & 0.0000170 & 0.0000169 & 0.0000168\end{array}$ $\begin{array}{lllllllllll}0.0000168 & 0.0000170 & 0.0000173 & 0.0000179 & 0.0000187 & 0.0000199 & 0.0000214 & 0.0000233\end{array}$ $\begin{array}{llllllllllll}0.0000257 & 0.0000287 & 0.0000324 & 0.0000368 & 0.0000422 & 0.0000486 & 0.0000562 & 0.0000653\end{array}$ $\begin{array}{lllllllllll}0.0000759 & 0.0000884 & 0.0001031 & 0.0001203 & 0.0001406 & 0.0001645 & 0.0001927 & 0.0002263\end{array}$ $\begin{array}{lllllllllll}0.0002664 & 0.0003145 & 0.0003726 & 0.0004433 & 0.0005298 & 0.0006364 & 0.0007688 & 0.0009343\end{array}$ $\begin{array}{llllllllll}0.0011429 & 0.0014078 & 0.0017469 & 0.0021846 & 0.0027537 & 0.0034990 & 0.0044801 & 0.0057751\end{array}$

Figure D1. (Sheet 3 of 6) 
$\begin{array}{llllllllll}0.0074806 & 0.0097054 & 0.0125507 & 0.0160685 & 0.0202028 & 0.0247232 & 0.0291852 & 0.0329370\end{array}$ $\begin{array}{llllllllllll}0.0352289 & 0.0354451 & 0.0333966 & 0.0295096 & 0.0246672 & 0.0198213 & 0.0156261 & 0.0123284\end{array}$ $\begin{array}{llllllllllll}0.0098846 & 0.0081272 & 0.0068762 & 0.0059843 & 0.0053438 & 0.0048801 & 0.0045425 & 0.0042957\end{array}$ $\begin{array}{llllllllllll}0.0041145 & 0.0039793 & 0.0038737 & 0.0037823 & 0.0036900 & 0.0035819 & 0.0034439 & 0.0032658\end{array}$ $\begin{array}{llllllllllll}0.0030429 & 0.0027787 & 0.0024847 & 0.0021781 & 0.0018772 & 0.0015974 & 0.0013486 & 0.0011351\end{array}$ $\begin{array}{lllllllllllllll}0.0009565 & 0.0008096 & 0.0006901 & 0.0005931 & 0.0005147 & 0.0004510 & 0.0003991 & 0.0003568\end{array}$ $\begin{array}{lllllllllll}0.0003220 & 0.0002933 & 0.0002696 & 0.0002500 & 0.0002338 & 0.0002204 & 0.0002095 & 0.0002006\end{array}$ $\begin{array}{llllllllllll}0.0001935 & 0.0001880 & 0.0001840 & 0.0001814 & 0.0001801 & 0.0001801 & 0.0001814 & 0.0001840\end{array}$ $\begin{array}{lllllllll}0.0001878 & 0.0001928 & 0.0001989 & 0.0002060 & 0.0002136 & 0.0002214 & 0.0002289 & 0.0002355\end{array}$ $\begin{array}{lllllll}0.0002409 & 0.0002445 & 0.0002463 & 0.0002461 & 0.0002448\end{array}$
$\begin{array}{lll}8 & 0.11279 & 2.089863\end{array}$
123456
20

$\begin{array}{lllllllllll}0.0000522 & 0.0000545 & 0.0000578 & 0.0000613 & 0.0000650 & 0.0000688 & 0.0000728 & 0.0000770\end{array}$ $\begin{array}{llllllllllllll}0.0000813 & 0.0000857 & 0.0000902 & 0.0000947 & 0.0000991 & 0.0001033 & 0.0001070 & 0.0001102\end{array}$ $\begin{array}{lllllllllll}0.0001124 & 0.0001136 & 0.0001133 & 0.0001116 & 0.0001084 & 0.0001037 & 0.0000978 & 0.0000909\end{array}$ $\begin{array}{lllllllllllll}0.0000836 & 0.0000761 & 0.0000688 & 0.0000620 & 0.0000557 & 0.0000502 & 0.0000453 & 0.0000410\end{array}$ $\begin{array}{lllllllllll}0.0000373 & 0.0000341 & 0.0000314 & 0.0000290 & 0.0000269 & 0.0000251 & 0.0000235 & 0.0000222\end{array}$ $\begin{array}{lllllllllll}0.0000210 & 0.0000199 & 0.0000190 & 0.0000182 & 0.0000174 & 0.0000168 & 0.0000163 & 0.0000158\end{array}$ $\begin{array}{lllllllllll}0.0000154 & 0.0000151 & 0.0000148 & 0.0000146 & 0.0000144 & 0.0000143 & 0.0000143 & 0.0000143\end{array}$ $\begin{array}{lllllllllll}0.0000143 & 0.0000145 & 0.0000146 & 0.0000149 & 0.0000152 & 0.0000157 & 0.0000162 & 0.0000169\end{array}$ $\begin{array}{llllllllllll}0.0000177 & 0.0000187 & 0.0000199 & 0.0000214 & 0.0000233 & 0.0000255 & 0.0000283 & 0.0000316\end{array}$ $\begin{array}{llllllllllll}0.0000357 & 0.0000406 & 0.0000464 & 0.0000534 & 0.0000616 & 0.0000712 & 0.0000824 & 0.0000952\end{array}$ $\begin{array}{llllllllllllll}0.0001099 & 0.0001266 & 0.0001455 & 0.0001667 & 0.0001907 & 0.0002178 & 0.0002484 & 0.0002833\end{array}$ $\begin{array}{lllllllllll}0.0003232 & 0.0003691 & 0.0004226 & 0.0004852 & 0.0005593 & 0.0006480 & 0.0007555 & 0.0008876\end{array}$ $\begin{array}{lllllllllllll}0.0010525 & 0.0012622 & 0.0015347 & 0.0018971 & 0.0023911 & 0.0030803 & 0.0040614 & 0.0054754\end{array}$ $\begin{array}{llllllllll}0.0075140 & 0.0104064 & 0.0143627 & 0.0194510 & 0.0254233 & 0.0315676 & 0.0367402 & 0.0396652\end{array}$ $\begin{array}{lllllllllll}0.0394968 & 0.0362911 & 0.0310396 & 0.0251414 & 0.0197374 & 0.0153873 & 0.0121588 & 0.0098676\end{array}$ $\begin{array}{lllllllllll}0.0082720 & 0.0071640 & 0.0063906 & 0.0058475 & 0.0054647 & 0.0051947 & 0.0050035 & 0.0048645\end{array}$ $\begin{array}{lllllllllll}0.0047535 & 0.0046468 & 0.0045192 & 0.0043456 & 0.0041053 & 0.0037877 & 0.0033978 & 0.0029579\end{array}$ $\begin{array}{lllllllllll}0.0025016 & 0.0020639 & 0.0016712 & 0.0013378 & 0.0010658 & 0.0008500 & 0.0006815 & 0.0005509\end{array}$ $\begin{array}{llllllllll}0.0004499 & 0.0003714 & 0.0003100 & 0.0002615 & 0.0002230 & 0.0001920 & 0.0001669 & 0.0001462\end{array}$ $\begin{array}{lllllllllllll}0.0001292 & 0.0001150 & 0.0001030 & 0.0000928 & 0.0000841 & 0.0000766 & 0.0000701 & 0.0000645\end{array}$ $\begin{array}{lllllllllllll}0.0000595 & 0.0000552 & 0.0000514 & 0.0000481 & 0.0000452 & 0.0000427 & 0.0000407 & 0.0000390\end{array}$ $\begin{array}{llllllllllll}0.0000377 & 0.0000369 & 0.0000364 & 0.0000363 & 0.0000366 & 0.0000373 & 0.0000383 & 0.0000397\end{array}$

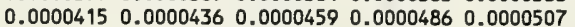

$$
9 \quad \begin{array}{llll}
0.12256 & 1.743782 & 123456 & 30
\end{array}
$$

$\begin{array}{llllllllllll}0.0000549 & 0.0000598 & 0.0000669 & 0.0000747 & 0.0000833 & 0.0000925 & 0.0001023 & 0.0001127\end{array}$ $\begin{array}{lllllllllll}0.0001235 & 0.0001346 & 0.0001456 & 0.0001563 & 0.0001661 & 0.0001747 & 0.0001814 & 0.0001856\end{array}$ $\begin{array}{lllllllllll}0.0001867 & 0.0001844 & 0.0001786 & 0.0001694 & 0.0001575 & 0.0001437 & 0.0001289 & 0.0001141\end{array}$ $\begin{array}{llllllllllll}0.0001001 & 0.0000873 & 0.0000760 & 0.0000663 & 0.0000580 & 0.0000511 & 0.0000454 & 0.0000406\end{array}$ $\begin{array}{lllllllllll}0.0000366 & 0.0000332 & 0.0000304 & 0.0000280 & 0.0000260 & 0.0000243 & 0.0000227 & 0.0000214\end{array}$ $\begin{array}{llllllllll}0.0000203 & 0.0000193 & 0.0000184 & 0.0000176 & 0.0000169 & 0.0000163 & 0.0000158 & 0.0000153\end{array}$ $\begin{array}{llllllllllll}0.0000149 & 0.0000146 & 0.0000143 & 0.0000141 & 0.0000139 & 0.0000138 & 0.0000137 & 0.0000137\end{array}$ $\begin{array}{llllllllllll}0.0000137 & 0.0000138 & 0.0000140 & 0.0000142 & 0.0000146 & 0.0000150 & 0.0000155 & 0.0000162\end{array}$ $\begin{array}{llllllllll}0.0000170 & 0.0000180 & 0.0000192 & 0.0000207 & 0.0000226 & 0.0000249 & 0.0000278 & 0.0000314\end{array}$ $\begin{array}{llllllllllll}0.0000358 & 0.0000414 & 0.0000483 & 0.0000569 & 0.0000675 & 0.0000806 & 0.0000967 & 0.0001162\end{array}$ $\begin{array}{llllllllllll}0.0001398 & 0.0001679 & 0.0002011 & 0.0002398 & 0.0002844 & 0.0003354 & 0.0003929 & 0.0004573\end{array}$ $\begin{array}{lllllllllll}0.0005287 & 0.0006074 & 0.0006935 & 0.0007872 & 0.0008887 & 0.0009984 & 0.0011170 & 0.0012455\end{array}$ $\begin{array}{llllllllll}0.0013862 & 0.0015430 & 0.0017229 & 0.0019370 & 0.0022034 & 0.0025495 & 0.0030166 & 0.0036650\end{array}$ $\begin{array}{lllllllllllll}0.0045809 & 0.0058839 & 0.0077314 & 0.0103134 & 0.0138240 & 0.0183871 & 0.0239147 & 0.0299027\end{array}$ $\begin{array}{lllllllllll}0.0352759 & 0.0385390 & 0.0384481 & 0.0348974 & 0.0291317 & 0.0229248 & 0.0175610 & 0.0135010\end{array}$ $\begin{array}{llllllllllll}0.0106514 & 0.0087278 & 0.0074557 & 0.0066303 & 0.0061146 & 0.0058204 & 0.0056909 & 0.0056872\end{array}$ $\begin{array}{lllllllllllll}0.0057777 & 0.0059286 & 0.0060951 & 0.0062163 & 0.0062167 & 0.0060227 & 0.0055930 & 0.0049464\end{array}$ $\begin{array}{lllllllllll}0.0041635 & 0.0033536 & 0.0026106 & 0.0019875 & 0.0014967 & 0.0011256 & 0.0008511 & 0.0006500\end{array}$ $\begin{array}{llllllllllll}0.0005027 & 0.0003941 & 0.0003134 & 0.0002526 & 0.0002062 & 0.0001704 & 0.0001425 & 0.0001203\end{array}$ $\begin{array}{llllllllllll}0.0001026 & 0.0000883 & 0.0000765 & 0.0000669 & 0.0000589 & 0.0000522 & 0.0000466 & 0.0000418\end{array}$ $\begin{array}{llllllllllll}0.0000378 & 0.0000345 & 0.0000316 & 0.0000293 & 0.0000274 & 0.0000259 & 0.0000247 & 0.0000239\end{array}$ $\begin{array}{lllllllllll}0.0000235 & 0.0000234 & 0.0000237 & 0.0000244 & 0.0000255 & 0.0000270 & 0.0000291 & 0.0000316\end{array}$ $0.00003470 .00003840 .0000427 \quad 0.00004770 .0000519$

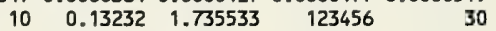

$\begin{array}{llllllllll}0.0000435 & 0.0000460 & 0.0000497 & 0.0000535 & 0.0000575 & 0.0000617 & 0.0000659 & 0.0000700\end{array}$ $\begin{array}{lllllllllllll}0.0000740 & 0.0000777 & 0.0000810 & 0.0000837 & 0.0000856 & 0.0000866 & 0.0000867 & 0.0000857\end{array}$ $\begin{array}{lllllllllll}0.0000837 & 0.0000806 & 0.0000768 & 0.0000722 & 0.0000673 & 0.0000622 & 0.0000572 & 0.0000525\end{array}$ $\begin{array}{lllllllllllll}0.0000481 & 0.0000443 & 0.0000410 & 0.0000383 & 0.0000360 & 0.0000341 & 0.0000327 & 0.0000315\end{array}$ $\begin{array}{lllllllllll}0.0000306 & 0.0000298 & 0.0000291 & 0.0000286 & 0.0000280 & 0.0000275 & 0.0000270 & 0.0000265\end{array}$ $\begin{array}{lllllllllll}0.0000259 & 0.0000253 & 0.0000246 & 0.0000239 & 0.0000232 & 0.0000225 & 0.0000218 & 0.0000210\end{array}$ $\begin{array}{lllllllllll}0.0000203 & 0.0000196 & 0.0000189 & 0.0000182 & 0.0000175 & 0.0000169 & 0.0000163 & 0.0000157\end{array}$ $\begin{array}{llllllllllll}0.0000152 & 0.0000148 & 0.0000143 & 0.0000140 & 0.0000137 & 0.0000135 & 0.0000133 & 0.0000132\end{array}$

Figure D1. (Sheet 4 of 6) 
$\begin{array}{llllllllll}0.0000132 & 0.0000134 & 0.0000136 & 0.0000140 & 0.0000146 & 0.0000154 & 0.0000164 & 0.0000178\end{array}$ $\begin{array}{lllllllllll}0.0000195 & 0.0000217 & 0.0000246 & 0.0000281 & 0.0000326 & 0.0000383 & 0.0000453 & 0.0000541\end{array}$ $\begin{array}{llllllllllll}0.0000650 & 0.0000783 & 0.0000946 & 0.0001143 & 0.0001378 & 0.0001656 & 0.0001982 & 0.0002361\end{array}$ $\begin{array}{llllllllllll}0.0002797 & 0.0003295 & 0.0003861 & 0.0004504 & 0.0005233 & 0.0006063 & 0.0007014 & 0.0008116\end{array}$ $\begin{array}{llllllllllll}0.0009415 & 0.0010979 & 0.0012914 & 0.0015387 & 0.0018661 & 0.0023157 & 0.0029549 & 0.0038897\end{array}$ $\begin{array}{llllllllllll}0.0052807 & 0.0073510 & 0.0103594 & 0.0144950 & 0.0196725 & 0.0253112 & 0.0303223 & 0.0334761\end{array}$ $\begin{array}{llllllllll}0.0339969 & 0.0319511 & 0.0281451 & 0.0236678 & 0.0194275 & 0.0159362 & 0.0133391 & 0.0115593\end{array}$ $\begin{array}{llllllllll}0.0104330 & 0.0097883 & 0.0094782 & 0.0093838 & 0.0094106 & 0.0094815 & 0.0095311 & 0.0095013\end{array}$ $\begin{array}{lllllllllll}0.0093408 & 0.0090080 & 0.0084785 & 0.0077556 & 0.0068767 & 0.0059096 & 0.0049365 & 0.0040313\end{array}$ $\begin{array}{lllllllllll}0.0032426 & 0.0025892 & 0.0020664 & 0.0016565 & 0.0013382 & 0.0010909 & 0.0008978 & 0.0007456\end{array}$ $\begin{array}{llllllllllll}0.0006243 & 0.0005266 & 0.0004469 & 0.0003813 & 0.0003267 & 0.0002808 & 0.0002420 & 0.0002089\end{array}$ $\begin{array}{lllllllllllll}0.0001805 & 0.0001561 & 0.0001351 & 0.0001169 & 0.0001012 & 0.0000877 & 0.0000761 & 0.0000662\end{array}$ $\begin{array}{lllllllllllllll}0.0000578 & 0.0000507 & 0.0000449 & 0.0000400 & 0.0000361 & 0.0000330 & 0.0000305 & 0.0000287\end{array}$ $\begin{array}{lllllllllll}0.0000275 & 0.0000267 & 0.0000264 & 0.0000264 & 0.0000269 & 0.0000277 & 0.0000289 & 0.0000304\end{array}$ 0.00003220 .00003440 .00003680 .00003960 .0000419

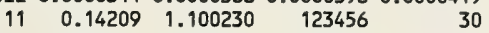

$\begin{array}{llllllllllll}0.0000484 & 0.0000502 & 0.0000527 & 0.0000555 & 0.0000584 & 0.0000614 & 0.0000647 & 0.0000681\end{array}$ $\begin{array}{llllllllllllll}0.0000716 & 0.0000753 & 0.0000791 & 0.0000829 & 0.0000867 & 0.0000905 & 0.0000942 & 0.0000976\end{array}$ $\begin{array}{llllllllll}0.0001007 & 0.0001033 & 0.0001053 & 0.0001065 & 0.0001069 & 0.0001063 & 0.0001047 & 0.0001022\end{array}$ $\begin{array}{lllllllllllll}0.0000987 & 0.0000946 & 0.0000898 & 0.0000847 & 0.0000794 & 0.0000741 & 0.0000689 & 0.0000640\end{array}$ $\begin{array}{lllllllllll}0.0000593 & 0.0000549 & 0.0000509 & 0.0000472 & 0.0000439 & 0.0000408 & 0.0000381 & 0.0000356\end{array}$ $\begin{array}{lllllllllll}0.0000333 & 0.0000313 & 0.0000294 & 0.0000277 & 0.0000262 & 0.0000248 & 0.0000235 & 0.0000223\end{array}$ $\begin{array}{lllllllllll}0.0000213 & 0.0000203 & 0.0000194 & 0.0000186 & 0.0000178 & 0.0000172 & 0.0000165 & 0.0000160\end{array}$ $\begin{array}{llllllllllll}0.0000155 & 0.0000151 & 0.0000147 & 0.0000144 & 0.0000142 & 0.0000140 & 0.0000139 & 0.0000139\end{array}$ $\begin{array}{lllllllllll}0.0000140 & 0.0000141 & 0.0000144 & 0.0000148 & 0.0000153 & 0.0000160 & 0.0000169 & 0.0000181\end{array}$ $\begin{array}{llllllllll}0.0000195 & 0.0000213 & 0.0000236 & 0.0000264 & 0.0000299 & 0.0000343 & 0.0000397 & 0.0000465\end{array}$ $\begin{array}{llllllllllllll}0.0000551 & 0.0000657 & 0.0000790 & 0.0000957 & 0.0001163 & 0.0001420 & 0.0001737 & 0.0002126\end{array}$ $\begin{array}{llllllllllll}0.0002602 & 0.0003183 & 0.0003886 & 0.0004736 & 0.0005762 & 0.0007000 & 0.0008497 & 0.0010317\end{array}$ $\begin{array}{llllllllllll}0.0012546 & 0.0015302 & 0.0018749 & 0.0023115 & 0.0028711 & 0.0035941 & 0.0045297 & 0.0057288\end{array}$ $\begin{array}{lllllllllll}0.0072275 & 0.0090181 & 0.0110173 & 0.0130539 & 0.0149006 & 0.0163467 & 0.0172711 & 0.0176723\end{array}$ $\begin{array}{llllllllllll}0.0176457 & 0.0173375 & 0.0169026 & 0.0164796 & 0.0161813 & 0.0160890 & 0.0162452 & 0.0166401\end{array}$ $\begin{array}{llllllllllll}0.0171933 & 0.0177510 & 0.0181138 & 0.0180967 & 0.0175961 & 0.0166213 & 0.0152771 & 0.0137139\end{array}$ $\begin{array}{lllllllllll}0.0120811 & 0.0104976 & 0.0090429 & 0.0077608 & 0.0066673 & 0.0057588 & 0.0050183 & 0.0044204\end{array}$ $\begin{array}{lllllllllllll}0.0039351 & 0.0035319 & 0.0031827 & 0.0028655 & 0.0025658 & 0.0022767 & 0.0019977 & 0.0017319\end{array}$ $\begin{array}{llllllllllllllll}0.0014839 & 0.0012575 & 0.0010552 & 0.0008781 & 0.0007256 & 0.0005961 & 0.0004877 & 0.0003978\end{array}$ $\begin{array}{lllllllllll}0.0003241 & 0.0002641 & 0.0002156 & 0.0001766 & 0.0001455 & 0.0001208 & 0.0001013 & 0.0000858\end{array}$ $\begin{array}{lllllllllllll}0.0000737 & 0.0000641 & 0.0000567 & 0.0000508 & 0.0000463 & 0.0000429 & 0.0000403 & 0.0000385\end{array}$ $\begin{array}{lllllllllll}0.0000372 & 0.0000364 & 0.0000360 & 0.0000360 & 0.0000364 & 0.0000370 & 0.0000379 & 0.0000390\end{array}$

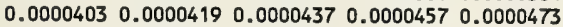

$$
\begin{array}{lllll}
12 & 0.15186 & 0.766675 & 123456 & 30
\end{array}
$$

$\begin{array}{lllllllllllll}0.0000492 & 0.0000525 & 0.0000574 & 0.0000632 & 0.0000698 & 0.0000772 & 0.0000854 & 0.0000941\end{array}$ $\begin{array}{llllllllllll}0.0001031 & 0.0001119 & 0.0001200 & 0.0001266 & 0.0001311 & 0.0001328 & 0.0001314 & 0.0001270\end{array}$ $\begin{array}{llllllllllll}0.0001198 & 0.0001106 & 0.0001001 & 0.0000891 & 0.0000785 & 0.0000686 & 0.0000598 & 0.0000522\end{array}$ $\begin{array}{llllllllllllll}0.0000459 & 0.0000406 & 0.0000363 & 0.0000329 & 0.0000302 & 0.0000282 & 0.0000267 & 0.0000256\end{array}$ $\begin{array}{llllllllllll}0.0000250 & 0.0000247 & 0.0000248 & 0.0000252 & 0.0000259 & 0.0000269 & 0.0000282 & 0.0000297\end{array}$ $\begin{array}{lllllllllll}0.0000315 & 0.0000335 & 0.0000356 & 0.0000377 & 0.0000398 & 0.0000418 & 0.0000436 & 0.0000450\end{array}$ $\begin{array}{lllllllllll}0.0000460 & 0.0000466 & 0.0000468 & 0.0000466 & 0.0000460 & 0.0000451 & 0.0000439 & 0.0000426\end{array}$ $\begin{array}{llllllllllllll}0.0000412 & 0.0000398 & 0.0000384 & 0.0000371 & 0.0000359 & 0.0000348 & 0.0000339 & 0.0000331\end{array}$ $\begin{array}{llllllllllll}0.0000326 & 0.0000322 & 0.0000321 & 0.0000322 & 0.0000326 & 0.0000332 & 0.0000342 & 0.0000356\end{array}$ $\begin{array}{lllllllllll}0.0000374 & 0.0000396 & 0.0000424 & 0.0000459 & 0.0000502 & 0.0000554 & 0.0000617 & 0.0000693\end{array}$ $\begin{array}{llllllllllll}0.0000785 & 0.0000897 & 0.0001032 & 0.0001196 & 0.0001397 & 0.0001642 & 0.0001943 & 0.0002316\end{array}$ $\begin{array}{llllllllllll}0.0002781 & 0.0003365 & 0.0004105 & 0.0005052 & 0.0006272 & 0.0007859 & 0.0009936 & 0.0012668\end{array}$ $\begin{array}{lllllllllllll}0.0016266 & 0.0020991 & 0.0027137 & 0.0034989 & 0.0044725 & 0.0056280 & 0.0069179 & 0.0082467\end{array}$ $\begin{array}{llllllllll}0.0094830 & 0.0104991 & 0.0112176 & 0.0116402 & 0.0118380 & 0.0119168 & 0.0119818 & 0.0121170\end{array}$ $\begin{array}{llllllllll}0.0123803 & 0.0128062 & 0.0134097 & 0.0141863 & 0.0151075 & 0.0161128 & 0.0171046 & 0.0179503\end{array}$ $\begin{array}{llllllllll}0.0185021 & 0.0186332 & 0.0182803 & 0.0174680 & 0.0162983 & 0.0149154 & 0.0134610 & 0.0120456\end{array}$ $\begin{array}{lllllllllllll}0.0107394 & 0.0095765 & 0.0085651 & 0.0076987 & 0.0069635 & 0.0063430 & 0.0058211 & 0.0053826\end{array}$ $\begin{array}{lllllllllllll}0.0050136 & 0.0047007 & 0.0044312 & 0.0041919 & 0.0039700 & 0.0037530 & 0.0035299 & 0.0032923\end{array}$ $\begin{array}{llllllllll}0.0030357 & 0.0027596 & 0.0024677 & 0.0021675 & 0.0018683 & 0.0015802 & 0.0013123 & 0.0010716\end{array}$ $\begin{array}{llllllllllll}0.0008623 & 0.0006857 & 0.0005405 & 0.0004240 & 0.0003321 & 0.0002607 & 0.0002059 & 0.0001641\end{array}$ $\begin{array}{llllllllllll}0.0001322 & 0.0001080 & 0.0000896 & 0.0000756 & 0.0000649 & 0.0000567 & 0.0000504 & 0.0000457\end{array}$ $\begin{array}{llllllllllll}0.0000421 & 0.0000394 & 0.0000375 & 0.0000363 & 0.0000356 & 0.0000354 & 0.0000358 & 0.0000366\end{array}$ $\begin{array}{llllll}0.0000378 & 0.0000396 & 0.0000419 & 0.0000448 & 0.0000473\end{array}$

$\begin{array}{llll}13 & 0.16162 & 0.821175 & 123456\end{array}$

$\begin{array}{lllllllll}0.0001081 & 0.0001140 & 0.0001224 & 0.0001312 & 0.0001399 & 0.0001480 & 0.0001548 & 0.0001595\end{array}$ $\begin{array}{lllllllllll}0.0001617 & 0.0001609 & 0.0001572 & 0.0001509 & 0.0001428 & 0.0001335 & 0.0001237 & 0.0001141\end{array}$ $\begin{array}{lllllllllll}0.0001050 & 0.0000968 & 0.0000894 & 0.0000829 & 0.0000772 & 0.0000724 & 0.0000681 & 0.0000645\end{array}$

Figure D1. (Sheet 5 of 6 ) 


\begin{abstract}
$\begin{array}{lllllllllll}0.0000613 & 0.0000586 & 0.0000562 & 0.0000541 & 0.0000523 & 0.0000508 & 0.0000495 & 0.0000484\end{array}$ $\begin{array}{lllllllllllll}0.0000474 & 0.0000467 & 0.0000462 & 0.0000458 & 0.0000457 & 0.0000457 & 0.0000461 & 0.0000466\end{array}$ $\begin{array}{lllllllllll}0.0000475 & 0.0000486 & 0.0000500 & 0.0000518 & 0.0000538 & 0.0000560 & 0.0000584 & 0.0000608\end{array}$ $\begin{array}{lllllllllll}0.0000630 & 0.0000649 & 0.0000663 & 0.0000671 & 0.0000673 & 0.0000669 & 0.0000659 & 0.0000644\end{array}$ $\begin{array}{lllllllllll}0.0000626 & 0.0000606 & 0.0000585 & 0.0000565 & 0.0000546 & 0.0000529 & 0.0000514 & 0.0000502\end{array}$ $\begin{array}{lllllllllll}0.0000494 & 0.0000488 & 0.0000486 & 0.0000489 & 0.0000495 & 0.0000506 & 0.0000522 & 0.0000544\end{array}$ $\begin{array}{llllllllllll}0.0000572 & 0.0000608 & 0.0000652 & 0.0000707 & 0.0000775 & 0.0000857 & 0.0000956 & 0.0001077\end{array}$ $\begin{array}{lllllllllllll}0.0001222 & 0.0001397 & 0.0001608 & 0.0001862 & 0.0002168 & 0.0002537 & 0.0002981 & 0.0003518\end{array}$ $\begin{array}{llllllllll}0.0004170 & 0.0004963 & 0.0005933 & 0.0007124 & 0.0008594 & 0.0010414 & 0.0012677 & 0.0015496\end{array}$ $\begin{array}{llllllllll}0.0019009 & 0.0023376 & 0.0028776 & 0.0035390 & 0.0043371 & 0.0052795 & 0.0063603 & 0.0075534\end{array}$ $\begin{array}{llllllllllll}0.0088095 & 0.0100597 & 0.0112281 & 0.0122521 & 0.0130998 & 0.0137782 & 0.0143272 & 0.0148053\end{array}$ $\begin{array}{lllllllll}0.0152720 & 0.0157746 & 0.0163404 & 0.0169703 & 0.0176342 & 0.0182660 & 0.0187629 & 0.0189973\end{array}$ $\begin{array}{llllllllll}0.0188423 & 0.0182143 & 0.0171133 & 0.0156352 & 0.0139437 & 0.0122162 & 0.0105969 & 0.0091740\end{array}$ $\begin{array}{lllllllll}0.0079824 & 0.0070199 & 0.0062640 & 0.0056847 & 0.0052516 & 0.0049370 & 0.0047163 & 0.0045670\end{array}$ $\begin{array}{llllllllll}0.0044679 & 0.0043971 & 0.0043316 & 0.0042476 & 0.0041230 & 0.0039409 & 0.0036936 & 0.0033854\end{array}$ $\begin{array}{llllllll}0.0030316 & 0.0026548 & 0.0022786 & 0.0019231 & 0.0016016 & 0.0013210 & 0.0010825 & 0.0008840\end{array}$ $\begin{array}{llllllllll}0.0007211 & 0.0005890 & 0.0004825 & 0.0003972 & 0.0003291 & 0.0002748 & 0.0002315 & 0.0001970\end{array}$ $\begin{array}{lllllllll}0.0001696 & 0.0001477 & 0.0001303 & 0.0001164 & 0.0001053 & 0.0000966 & 0.0000898 & 0.0000845\end{array}$ $\begin{array}{lllllllllllllll}0.0000806 & 0.0000778 & 0.0000761 & 0.0000752 & 0.0000753 & 0.0000761 & 0.0000778 & 0.0000804\end{array}$ $\begin{array}{lllll}0.0000837 & 0.0000880 & 0.0000932 & 0.0000993 & 0.0001044\end{array}$
\end{abstract}

Figure D1. (Sheet 6 of 6) 


\section{Appendix E \\ Notation}

Text Appendix C

$a_{0} \quad$ Normalizing coefficient in maximum likelihood estimate (MLE)

$a_{r}$ Normalizing coefficient for $r^{\text {th }}$ iteration in iterative maximum likelihood estimator (IMLE)

A Quartile asymmetry parameter angle(na)

Element na of an array that represents direction coordinates

$C_{i j}\left(f_{n}\right)$

Coincident spectral density between gauges $i$ and $j$ at frequency $f_{n}$

Water depth

datetime Ten-character string that contains date and time

dbar Mean water depth

$\operatorname{ddf}(n f, n a) \quad$ Array element representing the directional distribution function at frequency $f(n f)$ and direction angle(na)

$d \theta$

delang Direction increment

$d f$

delfs Frequency increment

dmax Maximum segment-averaged water depth in a collection 
Minimum segment-averaged water depth in a collection

$D\left(\theta_{m}\right)$

Directional distribution function based on $S\left(\theta_{m}\right)$

$D\left(f_{n}, \theta_{m}\right)$

Directional distribution function at frequency $f_{n}$ and direction $\theta_{m}$

$D_{0}\left(f_{n}, \theta_{m}\right)$

MLE estimate of directional distribution function at frequency $f_{n}$

$D_{r}\left(f_{n}, \theta_{m}\right)$

IMLE estimate of directional distribution function at frequency $f_{n}$ after $r^{\text {th }}$ iteration

$D_{r}^{\prime}\left(f_{n}, \theta_{m}\right)$

Intermediate, uncorrected IMLE estimate of directional distribution function at frequency $f_{n}$ during $r^{\text {th }}$ iteration

$\hat{\boldsymbol{e}}_{x}$

Unit vector in the $x$-direction

$\hat{\boldsymbol{e}}_{y}$

Unit vector in the $y$-direction

fds(nf,na)

$\mathrm{fp}$

$f_{p}$

g

Gravitational acceleration

$\operatorname{gpat}(n f)$

$h h m m$

Mnemonic for time of day

$H_{m o}$

Characteristic wave height tion spectrum at frequency $f(n f)$ and direction angle(na)

$n^{\text {th }}$ frequency of a set of $N$ discrete frequencies

Element $n f$ of an array that represents frequency

Peak frequency

Element nf of an array of six-character strings that represent working gauge patterns 


$$
i
$$

ifdtrnd

ifimle

ifwindo

istot

$i \operatorname{ter}(n f)$

$$
I\left(\theta_{m}-\theta_{m_{m n n}}\right)
$$

$\operatorname{Im}[]$

$j$

$k_{n}$

$$
\overrightarrow{\boldsymbol{k}}_{n}\left(\theta_{m}\right)
$$

$l$

$m$

$m_{1}$

$m_{2}$
Complex notation $\sqrt{-1}$ [in exponent or on main equation line]

Gauge index [as subscript]

Degrees of freedom in cross-spectral estimation

Flag indicating whether or not data have been detrended

Flag indicating if maximum likelihood or iterative maximum likelihood estimation is used

Flag indicating whether or not data segments have been windowed

Total number of seconds duration of a time series

Number of iterative maximum likelihood iterations used to compute directional distribution at frequency $f(n f)$

Number of gauges in an array

Cumulative distribution function

Imaginary part of complex entity contained in brackets

Gauge index [as subscript]

Magnitude of wave number vector associated with $n^{\text {th }}$ discrete frequency

Wave number vector for wave direction $\theta_{m}$ at $n^{\text {th }}$ discrete frequency

Summation index

na Index associated with discrete direction

First cosine moment of $D\left(\theta_{m}\right)$

Second cosine moment of $D\left(\theta_{m}\right)$ 


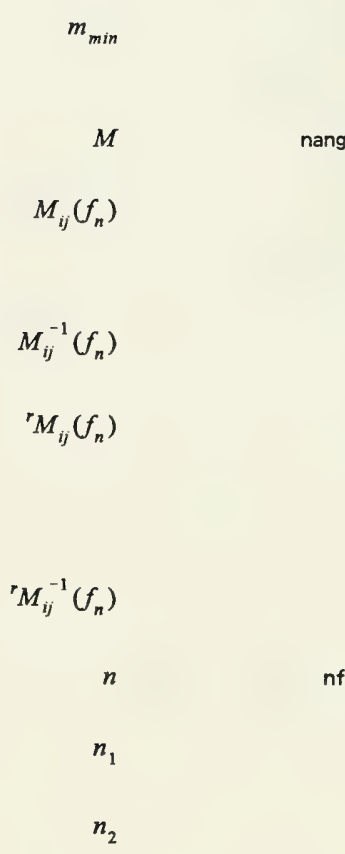

nband

nensb

nfft

$N$

$Q_{i j}\left(f_{n}\right)$

$r$

rname

$R$

$\operatorname{Re}[$ ]
Index of discrete direction at which wave energy is minimum

Integer number of discrete directions

Element of dimensionless matrix of cross spectra between gauges $i$ and $j$ at frequency $f_{n}$

Element of ịverse of $M_{i j}\left(f_{n}\right)$

Estimate of element of dimensionless matrix of cross spectra between gauges $i$ and $j$ at frequency $f_{n}$ during $r^{\text {th }}$ IMLE iteration

Element of inverse of ${ }^{r} M_{i j}\left(f_{n}\right)$

Index associated with discrete frequency

First sine moment of $D\left(\theta_{m}\right)$

Second sine moment of $D\left(\theta_{m}\right)$

Number of frequency bands averaged in spectral estimation

Number of segments into which a data record is divided during spectral estimation

Number of data points in a data segment

Integer number of discrete frequencies

Quadrature spectral density between gauges $i$ and $j$ at frequency $f_{n}$

Iteration count for IMLE

Five-character string denoting reference gauge

Upper limit of IMLE iterations

Real part of complex entity contained in brackets 
Text Appendix C

$s f(n f) \quad$ Element nf of an array that represents the frequency spectrum

sfrq Sampling frequency

$S\left(f_{n}\right)$

Frequency spectral density at frequency $f_{n}$

$S\left(\theta_{m}\right)$

Direction spectral density at direction $\theta_{m}$

$S\left(f_{n}, \theta_{m}\right)$

Frequency-direction spectral density at frequency $f_{n}$ and direction $\theta_{m}$

thp Peak direction of directional distribution at frequency $f p$

$T_{p}$

$x$

$\overrightarrow{\boldsymbol{x}}_{i}$

$\overrightarrow{\boldsymbol{x}}_{j}$

$y$

yymmdd

$\beta$

$\gamma$

$\Gamma_{i j}^{2}\left(f_{n}\right)$

$\delta$

$\Delta \theta$

$\epsilon_{r}$
Peak period

Horizontal coordinate increasing northward

Horizontal position vector of gauge $i$

Horizontal position vector of gauge $j$

Horizontal coordinate increasing westward

Mnemonic for date

Exponential convergence rate parameter in IMLE

Convergence rate coefficient in IMLE

Circular skewness

Coherence of signals from gauges $i$ and $j$ at frequency $f_{n}$

Circular kurtosis

Quartile directional spread parameter

Convergence check parameter at $r^{\text {th }}$ IMLE iteration 
Text Appendix C

$\begin{array}{cl}\theta_{0} & \text { Mean direction } \\ \theta_{25 \%} & \begin{array}{l}\text { First quartile direction of cumulative distribution } \\ \text { function }\end{array} \\ \theta_{50 \%} & \text { Median direction of cumulative distribution func- } \\ \theta_{75 \%} & \text { tion } \\ \theta_{l} & \begin{array}{l}\text { Third quartile direction of cumulative distribution } \\ \text { function }\end{array} \\ \theta_{m} & l^{\text {th }} \text { discrete direction } \\ \theta_{m_{\min }} & m^{\text {th }} \text { direction of a set of } M \text { discrete directions } \\ \theta_{p} & \text { Direction of minimum energy } \\ \phi_{i j}\left(f_{n}\right) & \text { Peak direction } \\ \left.\theta_{m}\right) & \text { Cross-spectral phase between gauges } i \text { and } \\ \text { frequency } f_{n}\end{array}$




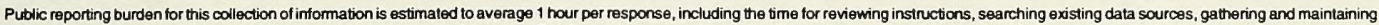

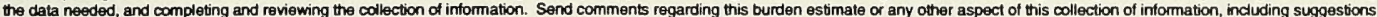

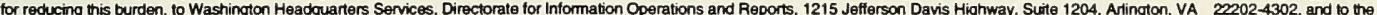
Office of Management and Budget, Paperwork Reduction Project (0704-0188), Washington, DC 20503.
1. AGENCY USE ONLY (Leave blank)
2. REPORT DATE June 1996
3. REPORT TYPE AND DATES COVERED
Final report

4. TITLE AND SUBTTILE

1994 Annual Index of Wind Wave Directional Spectra Measured at Harvest

Platform

6. AUTHOR(S)

Charles E. Long

5. FUNDING NUMBERS

7. PERFORMING ORGANIZATION NAME(S) AND ADDRESS(ES)

U.S. Army Engineer Waterways Experiment Station

3909 Halls Ferry Road REPORT NUMBER

Vicksburg, MS 39180-6199

Miscellaneous Paper CERC-96-4

9. SPONSORING/MONITORING AGENCY NAME(S) AND ADDRESS(ES)

U.S. Army Corps of Engineers

10. SPONSORING/MONITORING

Washington, DC 20314-1000

AGENCY REPORT NUMBER

11. SUPPLEMENTARY NOTES

Available from National Technical Information Service, 5285 Port Royal Road, Springfield, VA 22161.

12a. DISTRIBUTIONAVAILABILTTY STATEMENT

Approved for public release; distribution is unlimited.

12b. DISTRIBUTION CODE

13. ABSTRACT (Maximum 200 words)

This report indexes characteristic parameters of and describes a means of access to 2,320 wind wave frequency-direction spectra observed at the Texaco Oil Company Harvest Platform during calendar year 1994. Located at about the 200-m depth contour approximately $20 \mathrm{~km}$ west of Point Conception, California, the platform supports a spatial array of six pressure gauges, data from which are processed with an iterative maximum likelihood directional estimator. Nine parameters are defined, listed, and graphed in time series form: characteristic wave height, peak frequency, peak direction, four circular moments (mean direction, width, skewness, and kurtosis), and two parameters (directional spread and asymmetry) derived from quartile points of directional spectra. This report is the second in a series.

14. SUBJECT TERMS

Deep water

Frequency-direction spectra

Wave climate

Wind waves

15. NUMBER OF PAGES 118

16. PRICE CODE

17. SECURITY CLASSIFICATION OF REPORT

UNCLASSIFIED
18. SECURITY CLASSIFICATION OF THIS PAGE

UNCLASSIFIED
19. SECURITY CLASSIFICATION OF ABSTRACT 

Destroy this report when no longer needed. Do not return it to the originator. 


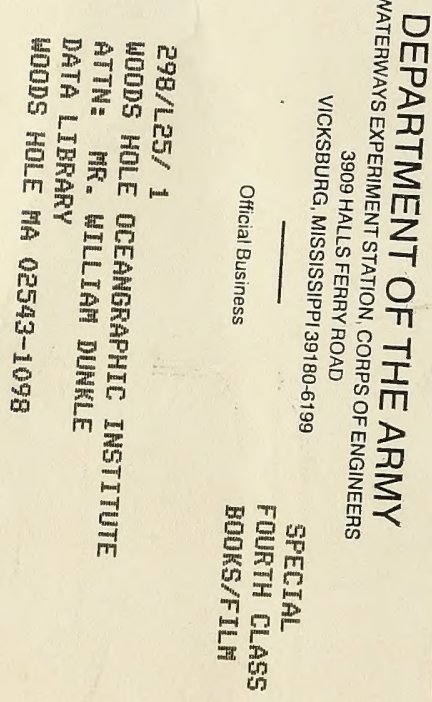

